\title{
HEALTH NEEDS ASSESSMENT: IMPACT ON PLANNING AND PURCHASING IN THE PUBLIC HEALTH SECTOR IN NEW ZEALAND
}

\author{
by \\ Gregor David Coster
}

\author{
A thesis \\ submitted to the Victoria University of Wellington \\ in fulfilment of the \\ requirements for the degree of \\ Doctor of Philosophy \\ in Public Policy
}

Victoria University of Wellington

2004 


\begin{abstract}
Health needs assessment (HNA) is one of the features of the New Zealand health system established by the New Zealand Public Health and Disability Act 2000. District Health Boards (DHBs) are to conduct HNAs, and planning of health services is intended to take into account the health needs of the population. Key questions for research relate to the impact of HNA on DHB planning and purchasing in a political/bureaucratic model of governance. This research was undertaken within a public policy framework that focused on evaluating the reforms against policy goals and expectations, and particularly against the influences that might be predicted from the HNA and prioritisation policy. Consideration was given to the range and effectiveness of past HNAs as well as the expectations and experiences of the DHB model in regard to HNA.
\end{abstract}

Document analysis and 34 interviews were conducted regarding 50 HNAs conducted in the public health sector from 1991-2000 to assess their impact on service delivery, decision-making, and policy. Document analysis was undertaken on DHB HNAs, prioritisation frameworks, board priorities, District Strategic Plans, and District Annual Plans for each of 20 DHBs. Planning and Funding managers were interviewed using semi-structured interview techniques to ascertain their experiences and views regarding the use of HNAs in planning. Grounded theory approaches were mainly used for the interview analysis. Case studies of five DHBs provided an in-depth understanding of the connections between health needs assessment, prioritisation, District Strategic Plans and District Annual Plans. Collection of contextual data provided an understanding of the influence of other policy decisions made locally or nationally. Using triangulation, conclusions were drawn regarding the effectiveness and impact of HNA and prioritisation on planning and health service purchasing by DHBs. The implications for public policy were then considered.

Recent needs assessments conducted by DHBs mostly met the minimum requirements of the Ministry of Health, but the quality was variable. DHB Planning and Funding Managers were unanimously positive regarding the usefulness of HNAs, and felt that there were good connections between them and the planning process (Connection Score). However, the impact of HNAs on planning and purchasing measured using 
document analysis (Impact Factor) was lower than expected. A number of barriers to effective use were identified. More focused HNA by DHBs is recommended with the use of mixed scanning approaches and service development groups directed towards specific service planning areas. Recommendations are made regarding future policy for HNA and prioritisation. 


\section{Acknowledgements}

This research was undertaken during my employment as the Elaine Gurr Professor of General Practice at the University of Auckland. I am grateful to the University for allowing me the opportunity to pursue this research.

I would like to thank my Supervisors, Professors Claudia Scott and Nicholas Mays, for their untiring support and advice, thoughtfulness and direction during this research. They have been unstinting in giving of their time and I am most appreciative. I could not have been more fortunate in my choice of supervisors.

Thanks are also due to Dr Chip Cangilose whilst he was Director of the Health Services Research Centre at Victoria University of Wellington for his support and advice as a supervisor in the early stages of this research. His return to the USA meant that he was no longer able to continue.

A number of others have provided advice and assistance. Dr Jackie Cumming and members of the Health Reforms 2001 Research project provided support and advice for the research for two years, prior to the author leaving the project.

Mark Booth in the Ministry of Health provided the initial opportunity for this research to take place when he commissioned me to write a background paper and literature review on health needs assessment for District Health Boards. I gratefully acknowledge the unknown peer reviewers who commented on that part of the work, but which was subsequently revised for this thesis.

I acknowledge with thanks the managers and researchers who willingly agreed to be interviewed regarding past health needs assessments. Their experience and insights were most valuable in obtaining a picture of health need assessment in the public sector over the decade 1991-2000. 
I acknowledge the two Ministry officials who agreed to share their insights regarding the reasons for, and expectations of, the health needs assessment and prioritisation policy. Theirs was a most useful contribution.

I would also like to acknowledge Dr Stephen Buetow, Senior Research Fellow, Department of General Practice and Primary Health Care, University of Auckland, for our discussions regarding the meaning of need and the subsequent paper that we published together on the subject.

The author is grateful to Prof David Wilkin for the suggestion to take a mathematical approach towards obtaining Impact Factors for the impact of health needs assessment on planning and purchasing.

Particular thanks are due to DHB Planning and Funding Managers throughout New Zealand who willingly gave of their time for their interviews and checked transcripts for accuracy. The insights that they shared were invaluable and this research could not have taken place without their willingness to participate. That they all agreed to do so is a tribute to the commitment of those people working in the public health sector in NZ.

Thanks to Diane Nicholson, my personal assistant, who ably assisted with some of the organisation of the research, and with the transcription of the tapes of interviews. Her help has been invaluable. Janet Hughes provided invaluable editing advice on the final drafts of the text.

Finally, but not least, my loving thanks and appreciation are due to Heather and the family who, with considerable patience, have put up with my commitment to the research and the long time that it has taken at all hours. 


\section{Preface}

This thesis studies health needs assessments and particularly the impact that they have had on DHB planning processes within the current health reforms. The research forms part of a larger research project entitled "Assessing governance, purchasing and accountability in the New Zealand health care sector". That project was funded by a Health Research Council Grant $(\$ 600,000)$ in the names of J Cumming, T Ashton, P Barnett, P Scott, C Kiro, C Cunningham, A Boulton, M Russell, T Tenbensel, M Powell, and G Coster. The project team subsequently collaborated with the Ministry of Health, Treasury and the State Services Commission, which together had obtained funding in the form of an Inter Departmental Contestable Pool Grant $(\$ 500,000)$ from the Ministry of Science, Research and Technology to conduct an evaluation of certain aspects of the DHBs. The project became known as the Health Reforms 2001 Research. I left the project team in February 2003 when I was appointed Chairman of the West Coast District Health Board.

My interest in this thesis research arose from multiple perspectives: interest in public policy and health services research, and a keen interest in community and health service need. The research provided the opportunity to combine those interests with evaluating DHBs, and contribute knowledge to the areas of health needs assessment, planning and purchasing. The topic also gave the opportunity to be involved in the wider health sector, particularly with the new DHBs, and in health policy. With this involvement came the possibility of influencing the direction of policy development. An invitation to prepare a background paper and literature review for the Ministry of Health and the DHBs led to the paper entitled 'Health Needs Assessment for New Zealand: Background Paper and Literature Review' (139pp.) which was sent by the Ministry to all DHBs to use as a reference source when preparing their health needs assessments (Coster, 2000). It also became the foundation of the literature review for this thesis. The evaluation of the impact of DHB health needs assessments was part of the evaluation of strategic decision-making within the Health Reforms 2001 project.

I have maintained my independence while conducting this research, and at the same time continued collegial relationships with the Health Reforms 2001 research group. The findings from my part of the research have been provided to the research group and will be incorporated into the final report. 


\section{TABLE OF CONTENTS}

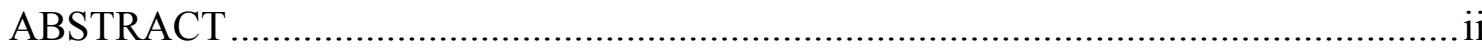

Acknowledgements ……………………………………………………………. iv

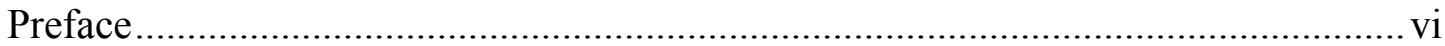

TABLE OF CONTENTS ....................................................................................... vii

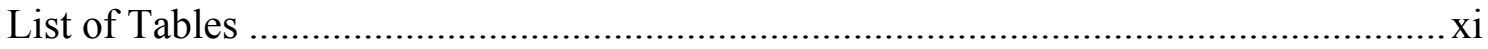

List of Figures .....................................................................................................

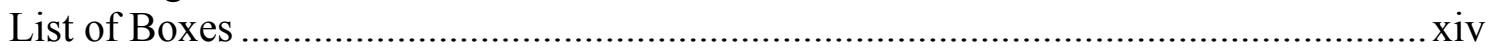

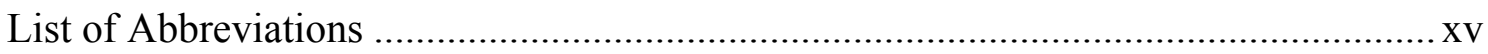

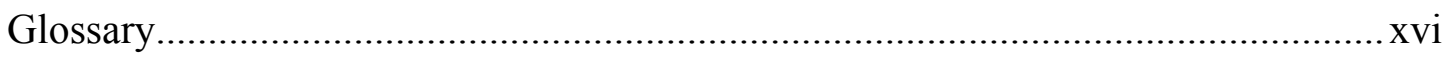

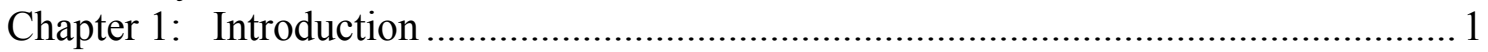

1.1 Background to research...............................................................................

1.2 Health Reforms 2001 Research......................................................................

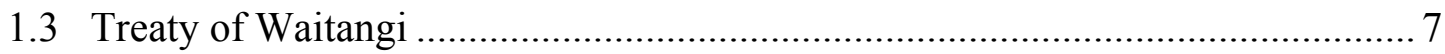

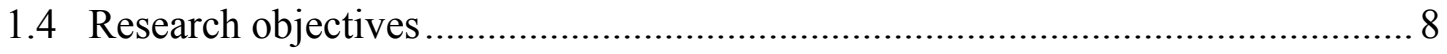

1.5 Research overview and thesis organisation.....................................................

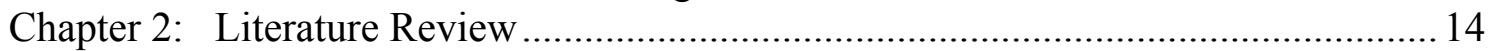

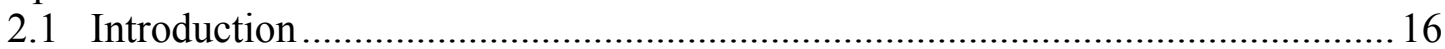

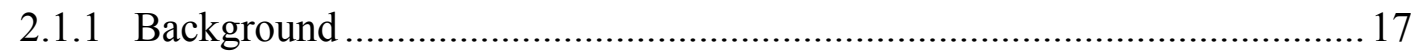

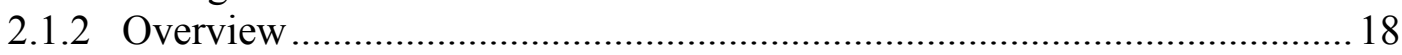

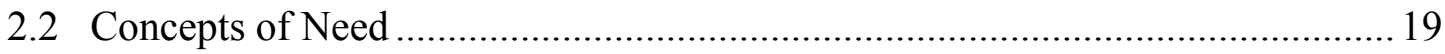

2.2.1 Introduction .................................................................................... 19

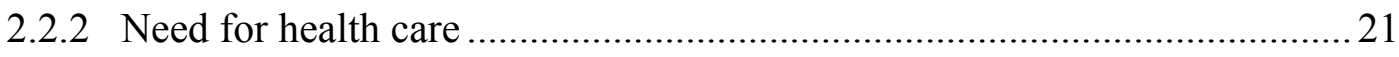

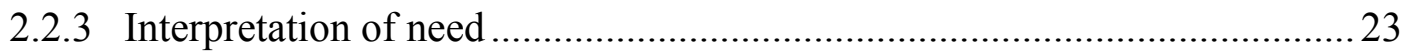

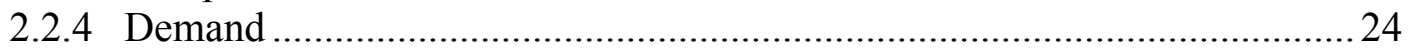

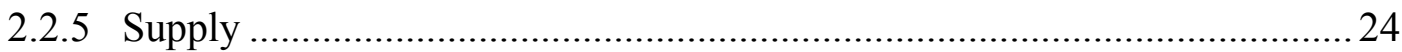

2.2.6 Need, demand and supply ................................................................... 25

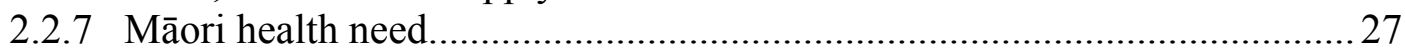

2.3 Health care needs assessment............................................................................ 30

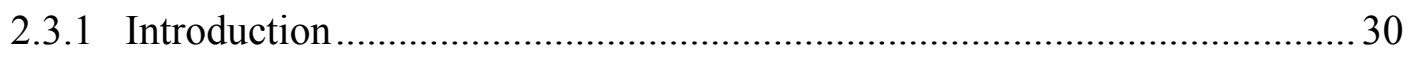

2.3.2 Purpose and objectives of HNAs …………………...................................... 31

2.3.3 Assessment methods used for HNA...........................................................

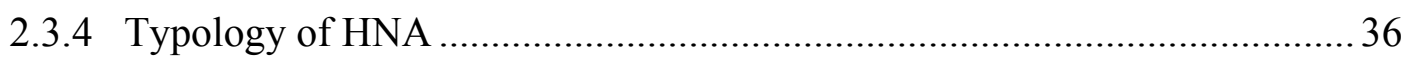

2.3.5 Requirements for HNAs to succeed ......................................................... 36

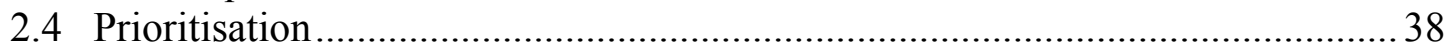

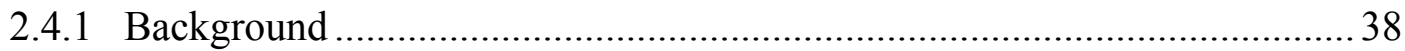

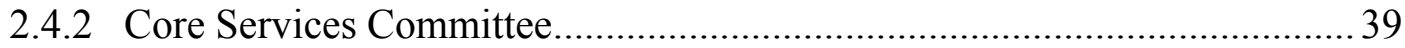

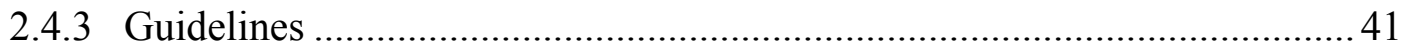

2.4.4 Health Funding Authority prioritisation process ………………………..... 42

2.4.5 National Health Committee review of the HFA process ............................... 44

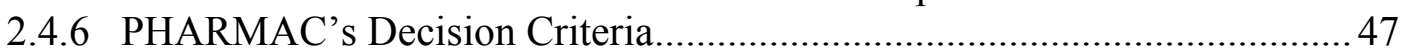

2.4.7 Prioritisation through ring fencing arrangements ....................................... 48

2.4.8 Programme budgeting and marginal analysis ............................................ 48

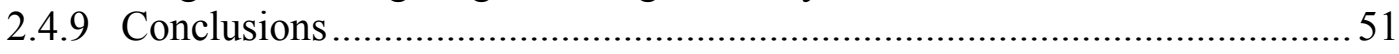

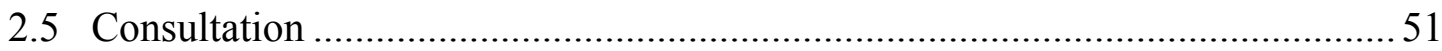

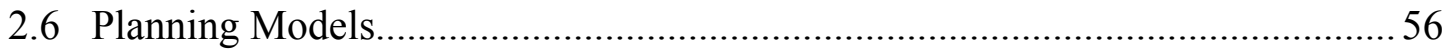

2.7 International perspectives regarding the impact of HNA on planning ................61

Chapter 3: Typology of Health Needs Assessments .........................................................68

3.1 Population-based approach ......................................................................... 70

3.2 Community-based approaches ………………………………………........ 70 


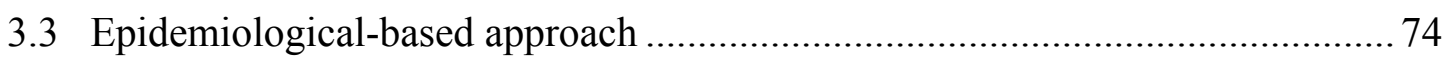

3.4 Comparative approach to needs assessment ................................................... 75

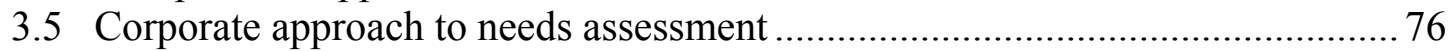

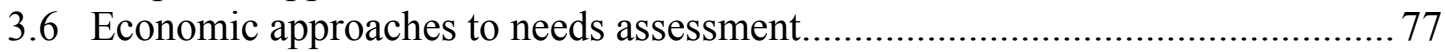

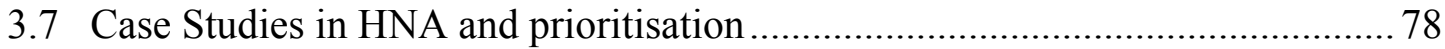

3.7.1 Porirua City Health and Disability Report and Plan - example of a

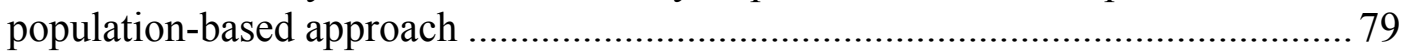

3.7.2 Rapid appraisal in an urban setting: an example from the developed world -

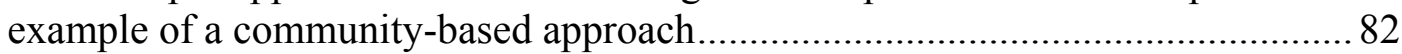

3.7.3 Renal Disease - example of an epidemiologically-based approach ............ 84

3.7.4 Economics, public health and health care purchasing: the Tayside experience of programme budgeting and marginal analysis - example of an

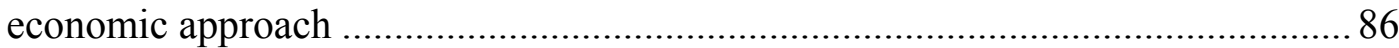

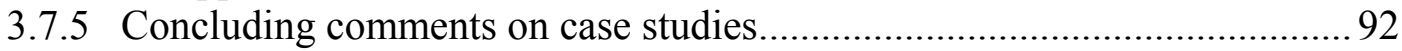

Chapter 4: Health Reforms: Policy Implications for Health Needs Assessment and

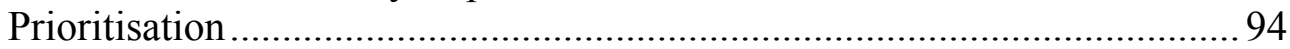

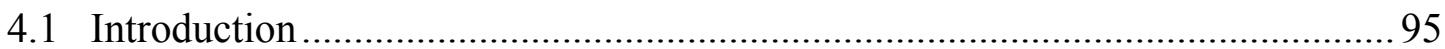

4.2 Area Health Boards (1983-93) ...................................................................... 95

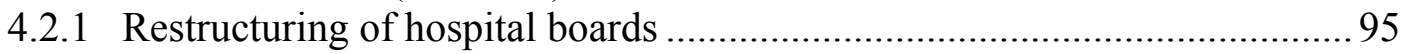

4.2.2 Evolution of health needs assessment and prioritisation in AHBs .............. 96

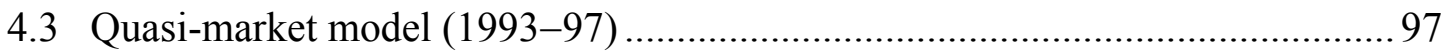

4.3.1 Reform from Area Health Boards to Regional Health Authorities...............97

4.3.2 Evolution of health needs assessment and prioritisation in RHAs ............. 99

4.4 Quasi-market model (1997-2000) .................................................................. 101

4.4.1 Reforms and the Health Funding Authority ........................................... 101

4.4.2 Implications for health needs assessment and prioritisation..................... 103

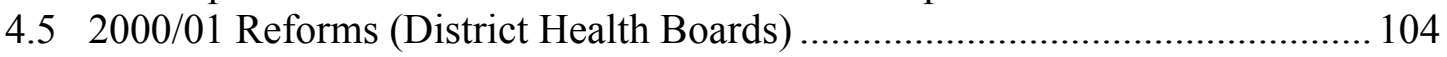

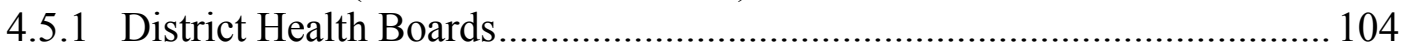

4.5.2 New Zealand Public Health and Disability Act 2000 ............................. 107

4.5.3 New Zealand Health Strategies................................................................... 109

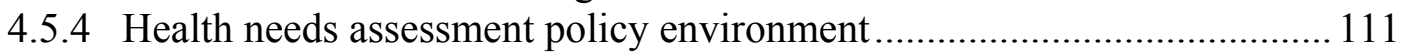

4.5.5 Implications for health needs assessment and prioritisation..................... 114

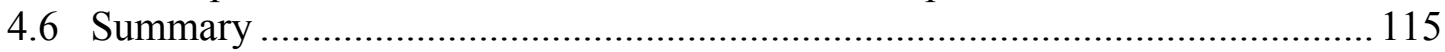

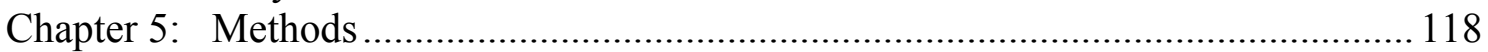

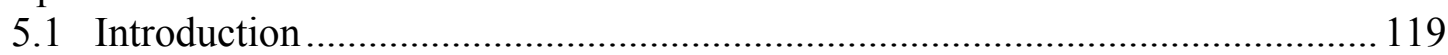

5.2 The application of qualitative methods to the current research ........................ 124

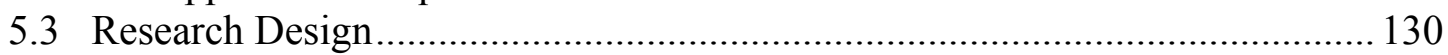

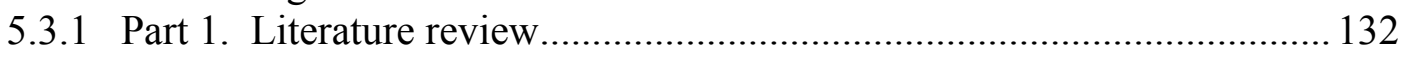

5.3.2 Part 2. Evaluation of past health needs assessments (1991-2000) ........... 133

5.3.3 Part 3. Evaluation of health needs assessments and prioritisation undertaken

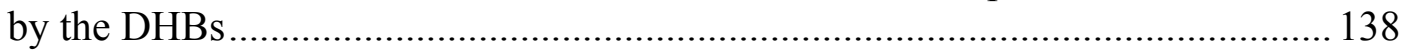

5.3.4 Part 4. Evaluation of the effectiveness of government policy regarding health needs assessment, prioritisation, and planning...................................... 147

Chapter 6: Past Needs Assessments (1991-2000) ............................................................ 149

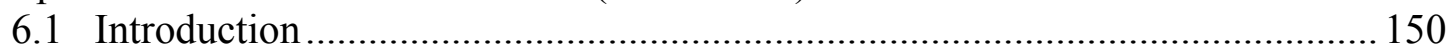

6.2 Overview of HNAs 1991-2000 ............................................................... 151

6.3 Overview of HNAs 1997-2000 ................................................................. 154

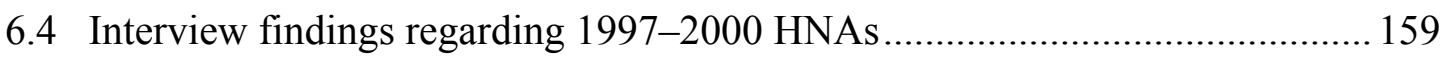

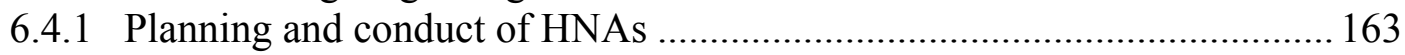

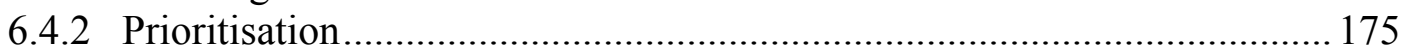

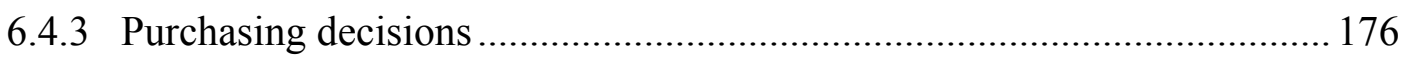

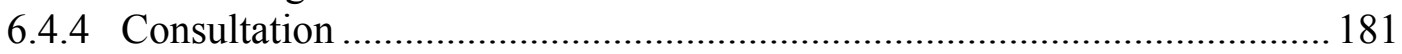

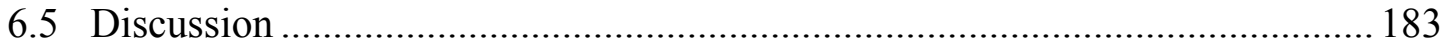


Chapter 7: District Health Boards Assessing Health Need .......................................... 189

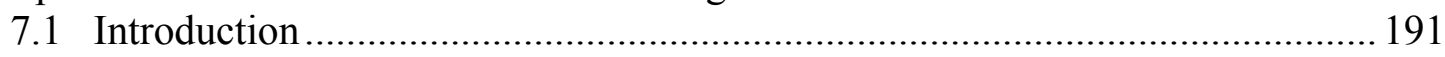

7.2 Transitional DHBs health needs assessment plans ......................................... 191

7.3 Health needs assessments by DHBs........................................................... 193

7.3.1 The Regional Health Needs Assessment Project (RHNAP) ...................... 193

7.3.2 Health needs assessments by other DHBs .................................................. 196

7.4 DHB health needs assessment document analysis ........................................... 197

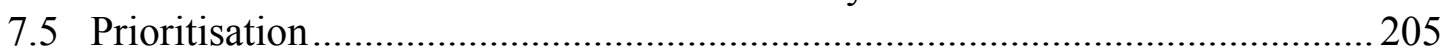

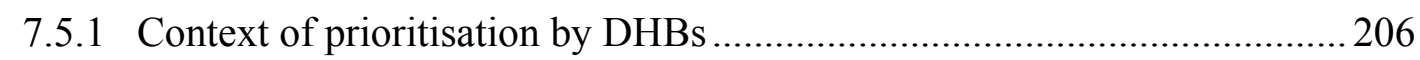

7.5.2 Prioritisation principles established by DHBs ........................................208

7.6 Planning and Funding Manager interviews..................................................... 211

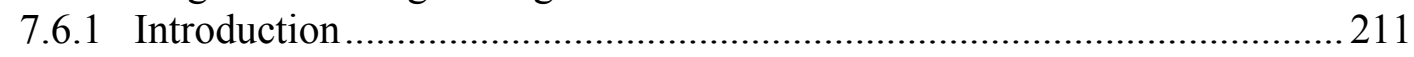

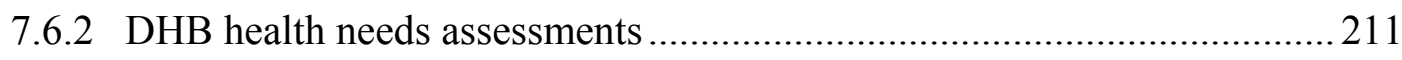

7.6.3 Prioritisation frameworks and prioritisation ............................................ 219

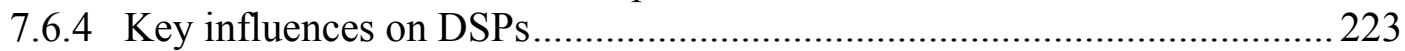

7.6.5 Linkages between DAPs and budgets .................................................... 225

7.6.6 Connections between health needs assessments and purchasing ...............226

7.7 Impact Factor - a measure of the impact of health needs assessments on

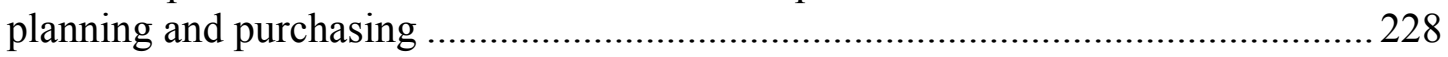

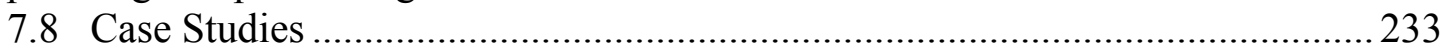

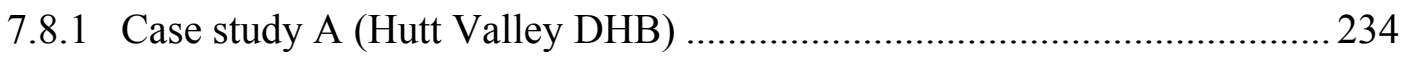

7.8.2 Case study B (Hawke's Bay DHB) ....................................................... 240

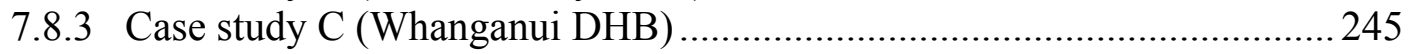

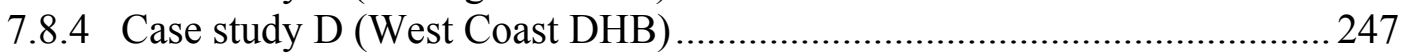

7.8.5 Case study E (South Canterbury DHB) .................................................. 249

7.9 Interviews with Ministry officials regarding expectations for health needs

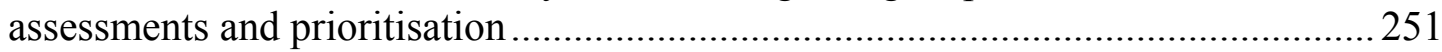

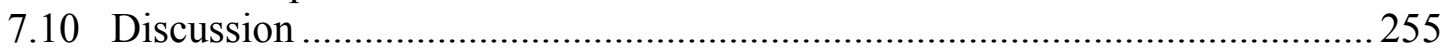

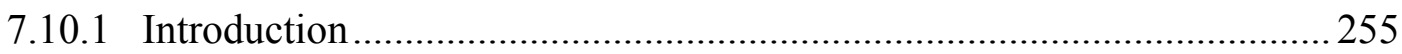

7.10.2 Types of DHB health needs assessment ............................................. 257

7.10.3 Impact of DHB health needs assessments............................................2.259

7.10.4 Most effective and least effective health needs assessments .................. 262

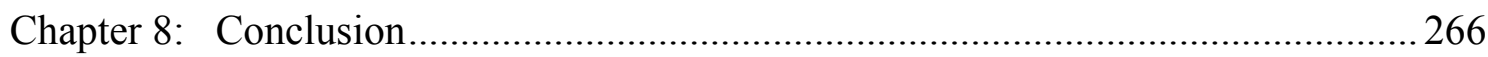

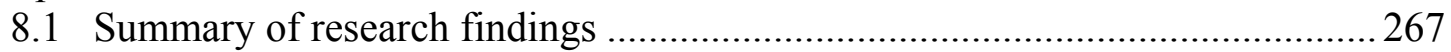

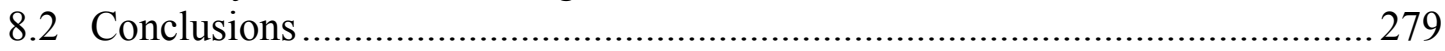

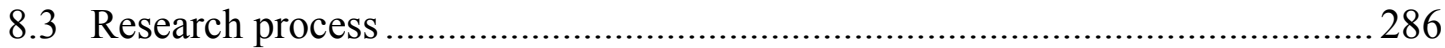

8.3.1 Strengths and Limitations of the research............................................. 286

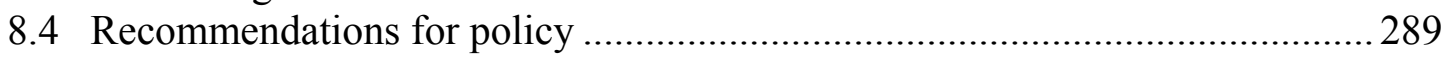

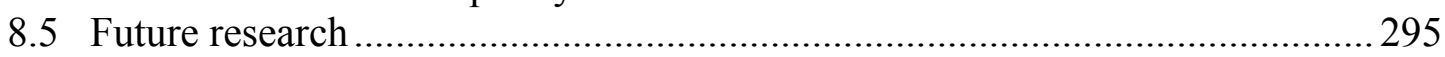

Appendix 1: District Health Boards, Populations and Geographical Areas ............ 296

Appendix 2: District Health Boards Map............................................................ 297

Appendix 3: Health Needs Assessments (1991-2000) .......................................... 298

Appendix 4: HNA Ethics Approval ........................................................................ 303

Appendix 5: Invitation letter to 1997-2000 interview participants ........................ 304

Appendix 6: Information Sheet (Past Interviews 1997-2000)............................... 306

Appendix 7: Consent form (Past Interviews 1997-2000)..................................... 308

Appendix 8: Semi-structured interview guide for interviews with HNA participants

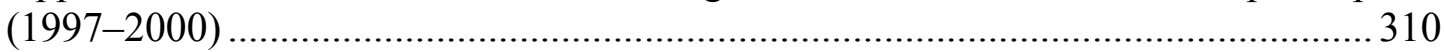

Appendix 9: Invitation letter to Planning and Funding Managers ........................... 311

Appendix 10: Information Letter for Planning and Funding Managers ................. 313

Appendix 11: Consent form for Planning and Funding Manager Interviews ......... 318

Appendix 12: Semi-structured interview guide for interviews with DHB funding and

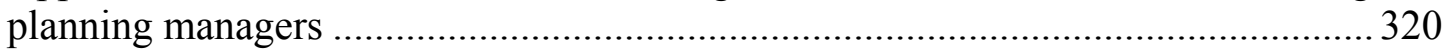


Appendix 13: DHB needs assessment recommendations, and priorities in district strategic and annual plans 322

Appendix 14: Review criteria template for evaluation of the Quality of DHB HNAs332

References. 


\section{List of Tables}

Table 1: Setting objectives for needs assessment exercises......................................... 32

Table 2: Approaches to consultation on health care priorities ...................................... 55

Table 3: Numbers and standardised rates of avoidable hospitalisations per 10,000

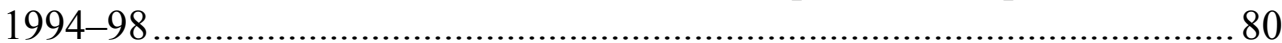

Table 4: Actions agreed for intersectoral action on health ...................................... 81

Table 5: Actions recommended to Agencies for intersectoral action .......................... 82

Table 6: Programme budget matrix for the Child Health Strategy in Tayside ..............90

Table 7: Top 10 suggested areas for service development and resource release, ranked by frequency of mention ........................................................................ 91

Table 8: Health reforms and effects on health needs assessment, prioritisation, including Māori and consumer involvement 1983-2003.

Table 9: Comprehensive rational planning model for DHBs and data sources available

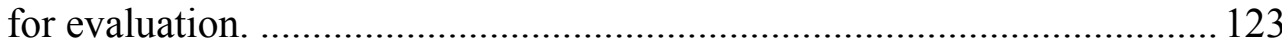

Table 10: Research methods used for each part of the evaluation programme. ......... 130

Table 11: Typological classification of health needs assessments.............................. 135

Table 12: Values assigned to three-digit numbers during analysis of impact of HNAs

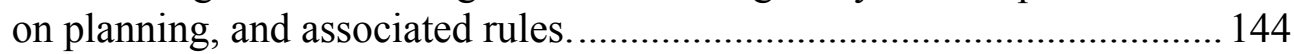

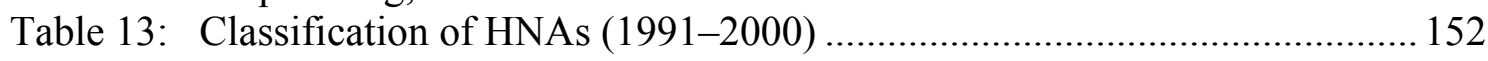

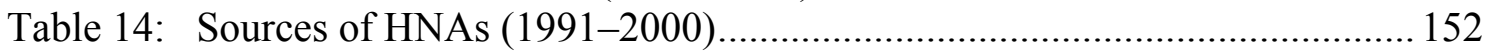

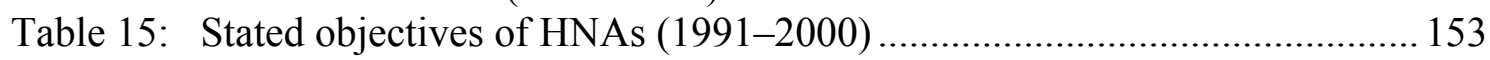

Table 16: Further breakdown of multiple objectives (refers to 'more than one of the

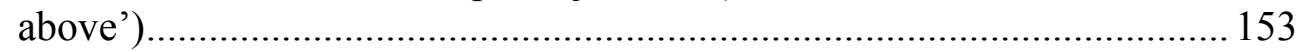

Table 17: Recommendations made to agencies (1991-2000) ...................................... 154

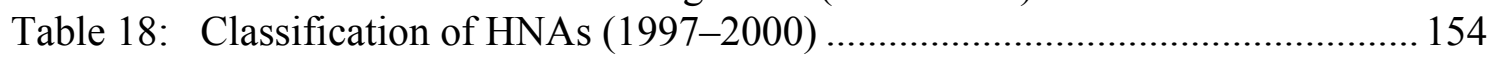

Table 19: Numbers of HNAs conducted by managers and researchers, for 1997-2000159

Table 20: Stated objectives of HNAs (1997-2000), comparing 1991-2000 data, and

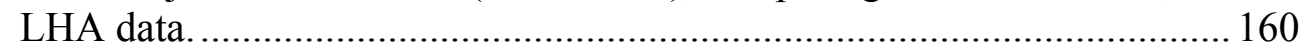

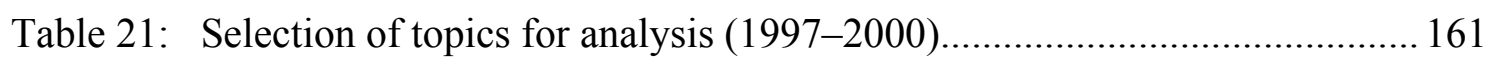

Table 22: Relationship between method by which a topic was selected for detailed HNA and subsequent impact of needs assessment on decision-making (1997-2000), compared to LHA data. .................................................... 162

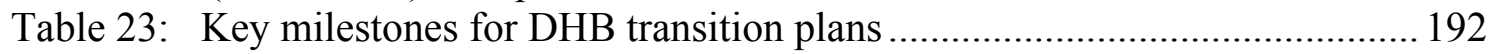

Table 24: Quality Scores for DHB HNAs.............................................................. 201

Table 25: Key health needs and need for services identified by District Health Boards

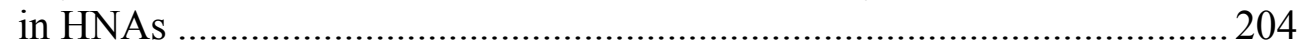

Table 26: Ranked frequency of key health needs and services identified by DHBs as

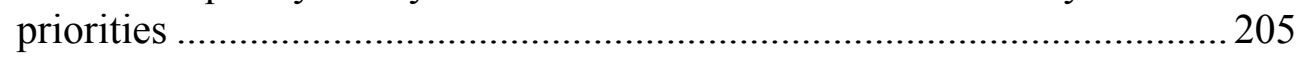

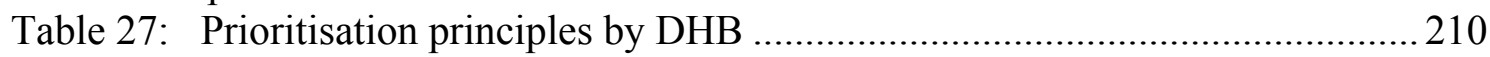

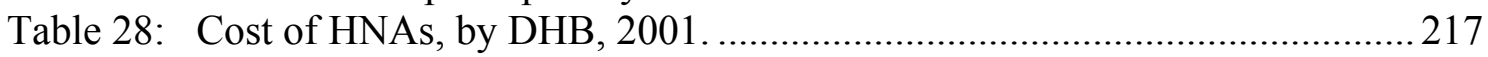

Table 29: Connection Scores for the steps from HNA to Purchasing (assessed using data from the DHB Planning and Funding Managers' interviews)............ 227

Table 30: Impact Factors, Connection Scores, HNA Quality Scores and DHB deficits as a percentage of budget. .....................................................................229

Table 31: Pearson Correlations for Impact Factors, Connection Scores, HNA Quality and DHB Deficits

Table 32: Activities undertaken by Case study DHBs in HNA, prioritisation and

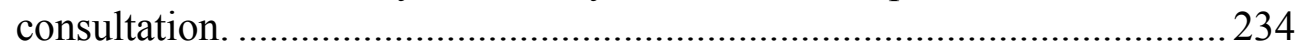

Table 33: Hutt Valley DHB service planning groups, and specific service plans (November 2001) 
Table A1: District Health Boards, populations and geographical areas....................2296

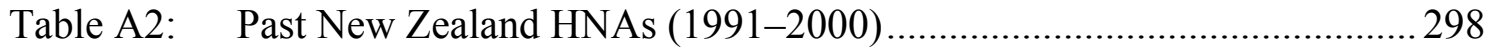




\section{List of Figures}

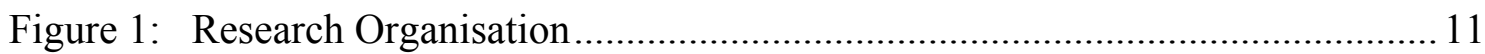

Figure 2: Interrelationship between need, supply and demand................................... 25

Figure 3: The gap in health outcomes between Māori and non-Māori ......................... 27

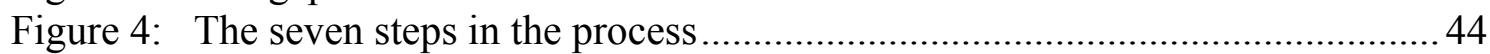

Figure 5: Contributions to the corporate view of local service needs.......................... 76

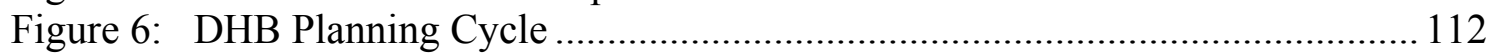

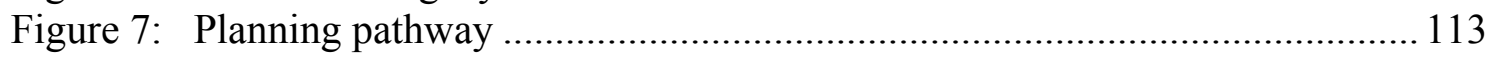

Figure 8: Comprehensive rational planning model applied to DHB health service

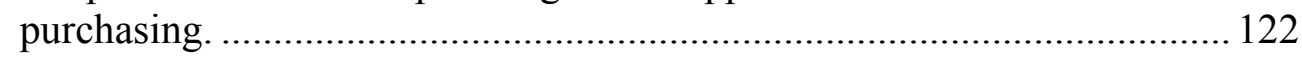

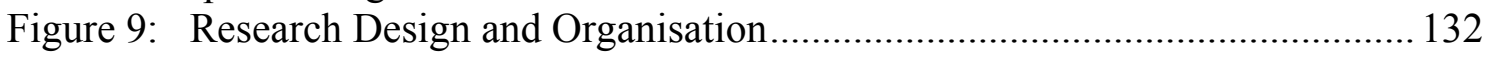

Figure 10: Example of the progression of HNA key recommendations into DHB Plans 142

Figure 11: Gantt Chart showing the timing relationships of the various components of the research.................................................................................... 148

Figure 12: Project Structure of the Regional HNA Project (RHNAP) ......................... 195

Figure 13: District Health Board responsibilities and the district health board planning

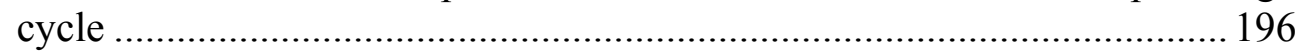

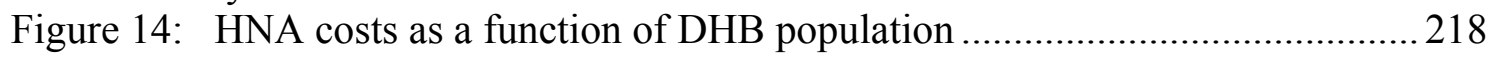

Figure 15: Relationship between Connection Scores and Impact Factors for DHB

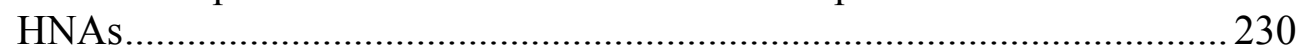

Figure 16: Connection Scores and Impact Factors as a function of DHB deficits \% of budget, Yr 2002/03 ........................................................................... 231

Figure 17: Relationship between Impact Factors and HNA quality scores ................. 232 


\section{List of Boxes}

Box 1: Goals for the pattern of provision of child health services in Tayside by 200587

Box 2: DHB Accountability Indicator GOV-02: Effective Health Needs Assessment139

Box 3: DHB Accountability Indicator GOV-03: Prioritisation ..................................... 141

Box 4: Ministry requirements for HNA plans in transitional DHB plans..................... 192

Box 5: Effective HNA Accountability Indicator for DHBs in 01/02........................... 199

Box 6: Prioritisation Accountability Indicator for DHBs in 02/03 ………................. 206 


\section{List of Abbreviations}

\begin{tabular}{|c|c|}
\hline AHB & Area Health Board \\
\hline $\mathrm{CEO}$ & Chief Executive Officer \\
\hline $\mathrm{CHE}$ & Crown Health Enterprise \\
\hline $\mathrm{CFO}$ & Chief Financial Officer \\
\hline $\mathrm{COO}$ & Chief Operating Officer \\
\hline CPHAC & Community and Public Health Advisory Committee \\
\hline CUA & Cost Utility Analysis \\
\hline CYFS & Children, Youth and Family Service \\
\hline DAP & District Annual Plan \\
\hline $\mathrm{DHB}$ & District Health Board \\
\hline DRG & Diagnostic Related Group \\
\hline DSP & District Strategic Plan \\
\hline ESRF & End Stage Renal Failure \\
\hline FFS & Fee-For-Service \\
\hline FMC & Funding Management Committee \\
\hline GP & General Practitioner \\
\hline HFA & Health Funding Authority \\
\hline HHS & Hospital and Health Services \\
\hline HNA & Health needs assessment \\
\hline ICD & International Classification of Diseases \\
\hline IDF & Inter District Flows \\
\hline IPA & Independent Practitioner Association \\
\hline MAPO & Māori Provider Organisations \\
\hline $\mathrm{MOH}$ & Ministry of Health \\
\hline MOU & Memorandum of Understanding \\
\hline NDOC & Non Departmental Output Class \\
\hline $\mathrm{NGO}$ & Non-Governmental Organisation \\
\hline $\mathrm{NHC}$ & National Health Committee \\
\hline NHS & National Health Service (England) \\
\hline NMDS & National Minimum Data Set \\
\hline NSF & National Service Framework \\
\hline NZHIS & New Zealand Health Information Service \\
\hline NZPHD Act & New Zealand Public Health and Disability Act 2000 \\
\hline OPF & Operating Policy Framework \\
\hline OPPs & Operating Policies and Procedures \\
\hline $\mathrm{PBFF}$ & Population Based Funding Formula \\
\hline PBMA & Programme Budgeting and Marginal Analysis \\
\hline PHARMAC & Pharmaceutical Management Agency \\
\hline PHC & Public Health Commission \\
\hline $\mathrm{PHO}$ & Primary Health Organisation \\
\hline QALYs & Quality-Adjusted Life Years \\
\hline RHA & Regional Health Authority \\
\hline RHNAP & Regional HNA Project \\
\hline RRT & Renal Replacement Therapy \\
\hline WINZ & Work and Income New Zealand \\
\hline
\end{tabular}




\section{Glossary}

(Where a definition has been sourced, the source is shown in brackets following the definition.)

Accessibility is the extent to which people are able to benefit from services on the basis of need, irrespective of socio-economic group, ethnicity, age, or gender. Access should be as fair as possible within the limits of available resources.

Acceptability refers to the ability to meet the wishes, desires and expectations of consumers.

Accountability Indicators refer to specific performance measures set by the Ministry of Health that must be met by DHBs.

Accreditation is a tool for monitoring organisational performance and for monitoring that the delivery of health care is conducted in an acceptable way - it involves peer review of organisational processes and systems.

Activities include all those action steps necessary to produce programme outputs.

Appropriateness refers to services that are considered suitable and proper for consumers of those services.

Budgetholding is granting a budget to a provider for a range of services over which they have control, and making them accountable for over-expenditure.

Capitation is a method of allocating resources to funders and providers based on a per capita payment for each service provider enrolled or located in a geographical district. Payment is not linked to the number of services provided to individual users. 
Clinical audit aims to raise performance, usually in one area, to meet local needs for the development of clinical practice.

Clinical governance has been defined in the UK as 'a framework through which the NHS organisations are accountable for continuously improving the quality of their services and safeguarding high standards of care by creating an environment in which excellence in clinical care will flourish' (Donaldson, 1998, p.33).

Community-based health needs assessments have the following characteristics and incorporate a high level of user and community involvement:

- are concerned with either health services or general social and environmental issues which affect health

- examine small areas or small population groupings

- involve work in the field

- adopt a flexible approach

- base the assessment largely on qualitative data derived from the perspectives of the local community (Ong, Humphris, Annett and Rifkin, 1991, summarised).

Comparative health needs assessments contrast the services received by the population in one area with those elsewhere, and can include a cost-effectiveness dimension.

Consumer participation describes the formal and informal processes through which individuals and communities are involved in and influence service planning, delivery, monitoring, and evaluation at all levels.

Core services is a list of those health services to which all New Zealanders would have access, on affordable terms and without unreasonable wait, through the publiclyfinanced health care system.

Corporate health needs assessments are based on the demands, wishes and alternative perspectives of interested parties, including political and public views.

Cost-effectiveness is when resources are concentrated on effective services, provided at the least cost, that offer the best payoff in terms of health dollar spent. 
Cost Utility Analysis (CUA) compares the costs and effects of interventions in terms of their usefulness in producing life years gained (PYLG), the quality of these life years (QALYs), or disability free years (DALYs).

Crown Funding Agreement is the agreement between a Crown entity (e.g. District Health Board, PHARMAC) and the Crown, that sets out the services the Crown is funding along with the conditions, performance and reporting measures that are to be in place in respect to the funded services.

Disability support services are services for people with disabilities, and their families and carers, to help them maintain their independence and well-being. Services include personal needs assessment services, service co-ordination, caregiver support including day care and respite care, rehabilitation information and advisory services for people with disabilities and their caregivers, environmental support and equipment, residential care, rest home and hospital care.

District Annual Plans (DAPs) are the DHB operational plans agreed annually between a DHB and the Ministry of Health.

District Strategic Plans (DSPs) are the DHB five yearly strategic plans agreed between a DHB and the Ministry of Health. DHBs must consult with the public on these plans before the Board signs them off.

Economic health needs assessments match information on the costs of health care interventions to the benefits produced so that purchasers can gain the greatest benefit from a defined budget.

Effectiveness refers to the extent to which health and disability services produce desired or preferred outcomes. 
Efficacy is the extent to which an intervention, when used under ideal conditions achieves the desired outcome.

Efficiency builds on effectiveness by considering costs as well as benefits. Efficiency recognises that resources are limited and choices have to be made between different services.

Enrolment is a process of actively joining an organisation (e.g. Primary Health Organisation) or provider (e.g. practice) in order to gain benefits linked to the organisation, or delivery of care for those who are enrolled.

Epidemiological-based health needs assessments are described in terms of disease rather than population groups or services. Descriptive epidemiology (as opposed to analytical epidemiology) is used to describe the occurrence of disease in terms of person, place, and time. Both costs and cost-effectiveness are generally taken into account.

Equity focuses on fair distribution of services in relation to people's needs, and their ability to benefit from services.

Funding Management Committee is a committee consisting of senior management (Chief Executive Officer, Chief Financial Officer, Chief Operating Officer) and sometimes the Board Chairman, that decides prioritisation of available funding, and makes recommendations to the Board.

Grounded theory - hypothesising inductively from data, notably using subjects' own categories, concepts etc.

Health describes the ability of individuals and populations to 'function in their environment by developing physical, psychological and spiritual resources for living' (Buetow and Kerse, 2001, p.73). 
Health assessment is a description of the health status of the population, using epidemiological methods. It is otherwise known as a health profile. This does not equate to a health needs assessment, which has a greater focus on the health needs of the population.

Health care needs assessment (HNA) refers to the 'assessment of the population's capacity to benefit from health care services, prioritised according to effectiveness, including cost-effectiveness, and funded within available resource' (Coster, 2000, p.2).

Health Funding Authority (HFA) was the principal funder of health and disability services in New Zealand between 1998-2000. A crown agency, the HFA received its funding from the Government.

Health gain refers to improvement of health status and independence for a population.

Health gain priority areas are those for which the Government specifically seeks to improve health status and promote independence. These are specifically described in the New Zealand Health Strategy.

Health profile is a description of the health status of the population, using epidemiological methods. It is otherwise known as a health assessment. This does not equate to a health needs assessment, which has a greater focus on the health needs of the population.

Health outcome is a change in the health status of an individual, group or population that is attributable to a planned programme or series of programmes, regardless of whether such a programme was intended to change health status.

Independent Practitioner Associations (IPAs) in New Zealand are umbrella organisations representing groups of general practitioners that negotiate budgets and contracts with purchasers on behalf of GPs. 
Induction is the process of moving from observations/data, towards generalisations, hypotheses, or theory (Mays and Pope, 1997); opposite of deduction, process of data gathering to test predefined theory or hypotheses.

Inter District Flows (IDFs) take account of the costs of delivering health services by a DHB for patients from another DHB. An IDF occurs when the DHB that has provided the service is paid by the DHB where the patient normally resides.

Kaumatua are Māori elders respected by Māori communities.

Medical case weights refer to the weighting assigned a discharge diagnosis based on length of stay averaged for all similar admissions. Medical case weights fall as duration of inpatient stay reduces.

National Health Committee (initially known as the Core Services Committee) was established in 1992 to advise the Minister of Health regarding the access by New Zealanders to publicly funded health services. Its full title is the National Advisory Committee on Core Health and Disability Support Services.

National Service Framework (NSF) is a collection of definitions, methodologies and processes that allow the sector to use common language when analysing, funding and monitoring services. The NSF includes (but is not limited to) decisionmaking/prioritisation processes, consultation guidelines, standard service agreement forms, defined service units, national service agreement monitoring and risk monitoring processes.

Outcomes can be described as effects, changes, benefits, consequences or impacts resulting from activities and outputs. (Short-term outcomes are those most closely associated or 'caused' by the programmes outputs. Intermediate outcomes are those that occur during the implementation of the programme, whereas long-term outcomes or impacts are the result of the final application of the whole programme.)

Outputs are the products, goods and services provided from programmes. 
Operational Policy Framework is a framework provided to DHBs each year by the Ministry of Health and sets out the operational policies under which DHBs must act.

Population-Based Funding Formula (PBFF) is a formula to distribute health funding among DHBs. It takes account of gender, ethnicity, socio-economic deprivation and rurality. Some boards also receive 'one-line adjusters' for to account for provision of tertiary services.

Population-based health needs assessments assess the overall health care need in a large population or district.

Primary health care means essential health care based on practical, scientifically sound, culturally appropriate and socially acceptable methods. It is universally accessible to people in their communities, involves community participation, is integral to, and a central function of, the country's health system, and is the first level of contact with the health system.

Prioritisation is a rigorous, explicit, ethical and transparent process based on an agreed set of principles to guide setting of priorities and the allocation of resources. It has also been defined as a decision-making process that results in resources (money, people etc) going to one set of services and not another.

Prioritisation framework refers to a framework that includes prioritisation principles for prioritisation of health services for purchasing.

Programme budgeting and marginal analysis (PBMA) is a health economic approach to prioritisation. Programme budgeting describes the programme and related budget, while marginal analysis evaluates where gains can be made at the margin.

Provider is a health professional or institution that delivers services to the service user. 
Provider arm refers to the DHB provider arm that is that part of the DHB that is responsible for providing services i.e. hospital-based services.

Quality of care can be defined generically for individual consumers in terms of 'whether individuals can access the health structures and processes of care which they need and whether the care received is effective' (Campbell, Roland and Buetow, 2000, p.1614).

Quality assessment seeks to furnish the information required to identify gaps in performance and opportunities to improve quality.

Quality assurance seeks to systematically identify outliers (e.g. inappropriate care), rectify them (rather than achieve error-free care), and maintain improvements.

Quality improvement is synonymous with continuous quality improvement, and is a culture or philosophy for "designing quality in" rather than "inspecting quality out".

Quality-adjusted life year (QALY) is a year of life adjusted for the health-related quality of that year. The quality adjustment is not straightforward as so many factors affect the quality of life. A number of systems for measuring health-related quality of life have been developed, but all have the following in common.

- A person in good health for age has a health-related quality of life of 1 .

- A dead person has a health-related quality of life of 0 .

- All intermediate health states have quality scores that lie between 1 and 0 .

Resources include human and financial resources as well as other inputs required to support a programme.

Risk management is identifying kinds and ranges of risks, managing certain events and ensuring an appropriate level of control and resources. 
Safety of health care is the extent to which harm from care by omission or commission or from the environment in which care is carried out is kept to a minimum.

Secondary care is care that is normally accessed following referral from a primary care provider, and is usually delivered in hospitals.

Service Coverage Schedule (SCS) is a statement of the minimum range of services that the government expects to be available for New Zealanders. It translates government policy into a required minimum range and standard of services.

Service planning group is a group of managers, providers, Māori providers, Iwi and community representatives given the task of planning a defined service or programme.

Standard is an object or quality or measure serving as a basis or example or principle to which providers of health and disability services conform or should conform or by which the accuracy or quality of their services is judged.

Structure is the relatively stable characteristics of the providers of health and disability services, of the tools and resources at their disposal, and of the physical and organisational settings in which they work including human, physical and financial resources (Donabedian, 1980).

Technical efficiency is when effective services are provided at least resource cost.

Volume is the quantity of provider output being funded and/or delivered (MOH). 
Chapter 1: Introduction

1.1 Background to research

1.2 Health Reforms 2001 Research

1.3 Treaty of Waitangi

1.4 Research objectives

1.5 Research overview and thesis organisation 


\subsection{Background to research}

A Labour-Alliance Coalition Government, elected in November 1999, reformed the health sector, introducing the New Zealand Public Health and Disability Bill in August 2000. The new Act providing for District Health Boards (DHBs) came into effect on 1 January 2001. The boards own public hospitals as their provider arms and fund other health services for the populations that they serve. This reform signalled an end to separation of purchase and provision for publicly delivered hospital services. The changes were intended to produce a better health service with more vertical integration.

The new legislation changed the legal form of public hospitals from companies to statutory entities. It provided for 21 Crown-owned, not-for-profit District Health Boards based on existing Hospital and Health Services (HHS) Boards. Supplementing these HHS Boards with appointed community members created transitional DHBs. After the local body elections in October 2001, DHBs each consisted of seven elected members, and up to four appointed members, to obtain a desirable balance of experience and community representation. The new legislation provided for special representation of Māori on DHBs and advisory committees, acknowledging obligations under the Treaty of Waitangi (see later). One of three statutory committees within each DHB is the Community and Public Health Advisory Committee, responsible to the board for health needs assessments (HNAs), including prioritisation.

The move to DHBs signalled a significant shift with respect to governance, purchasing and accountability. It involved an emphasis on meeting the needs of local populations, and consultation with communities on how those needs were to be met. There was also a requirement in the legislation for increased recognition of the Treaty of Waitangi in health care, by including specific Treaty provisions, and specific references to Māori in the Act.

DHBs were to be responsible for providing health and disability support services for the resident population in the district. The Ministry of Health, whilst encouraging local 
control of services by the DHBs, was to maintain some control of the delivery of health services. The two loci of control set up an interesting central-local dynamic with tension between central Ministry control and local democratic input. In July 2001, the Ministry of Health devolved purchasing for personal, primary, mental and Māori health to DHBs. Decisions regarding devolution of purchasing of public health and disability support services were deferred. Disability services were subsequently devolved to DHBs in October 2003.

\section{Health Needs Assessments}

The Health Funding Authority (HFA) and its predecessors, Regional Health Authorities (RHAs), frequently conducted HNAs, despite having no statutory requirement to do so. In January 2000, the Cabinet Business Committee agreed that this would become an obligatory function of DHBs (Minister of Health, 2000c). The HFA and the Ministry of Health formed a project team to prepare DHBs for HNAs. Two publications resulted from this workstream, the first of which forms the basis of the literature review for this research (Coster, 2000, Ministry of Health, 2000b).

With respect to HNAs in New Zealand, community and public health advisory committees of the board of a DHB are required to advise the board on:

(a) the needs, and any factors that the committee believes may adversely affect the health status, of the resident population of the DHB; and

(b) priorities for use of the health funding provided. (Schedule 4(2) (New Zealand Government, 2000, p.111))

DHBs were to be responsible for conducting HNAs for their districts, but could use shared services to do so. Requirements for the conduct of the HNA process were defined by the Ministry of Health (Ministry of Health, 2000b). Prioritisation was required to be consistent with the New Zealand Health Strategy (Minister of Health, 2000d) and the New Zealand Disability Strategy (Minister of Health, 2001b), and to take notice of multiple other health strategies published by the Government. 
The term 'HNA' covers a wide range of activities. Critical concepts and dimensions have been described by a number of authors (Harris and Marshall, 1997, p. 3-13, Stevens and Gabbay, 1991, Stevens and Raftery, 1994, Wright, Williams and Wilkinson, 1998a). The purpose of HNA is to produce systematically the knowledge required to inform changes in health care that are beneficial to the health of a population.

The various approaches to HNAs fit into two broad groups: population-based HNAs, which use epidemiological and quantitative methods to estimate the dimensions of need; and community-based needs assessments, which use primarily qualitative methods, based heavily on the perceptions of a defined community (Hensher and Fulop, 1999). A recently published series advises on practical approaches and methods of health need assessment (Stevens and Gillam, 1998b, Wilkinson and Murray, 1998, Williams and Wright, 1998).

The effectiveness of health needs assessment (HNA) needs exploring. Whether different approaches to HNA and its use in the planning process better promote strategic decision-making were key questions for this research. There was also interest in whether DHBs were able to shift resource to meet identified need better, or whether they were constrained by centrally determined health service priorities.

It was logical to begin by evaluating HNAs previously conducted in the public health sector by the Ministry of Health, the HFA and its predecessors. A ten-year period was chosen to give a reasonable review, dating from the previous Area Health Boards to disestablishment of the HFA in 2000. Given that many people in the health sector had changed jobs, or were otherwise lost to the sector over the years of rapid reform, to a sample period of four years seemed reasonable for in-depth interviews. DHB HNAs were then evaluated using a policy evaluation framework that is described in detail later in this thesis.

This research draws together thinking from many disciplines. The context for the research was the evaluation of the effectiveness of public policy. It utilised evaluation 
methodology and public policy disciplines. In the area of HNA the disciplines of public health, epidemiology, statistics, health economics, and social sciences were drawn upon for data collection and analysis. Social science has much to contribute regarding the meaning of health need, and the issues to do with need, supply and demand.

I expected that this research would find that HNA for DHBs would not have a significant impact on planning and purchasing for a number of reasons, mostly described in a paper by Wright et al (1998a). They concluded that HNAs fail for three main reasons: firstly, because the assessing of health needs is not properly understood, resulting in flawed methods; secondly, because of a lack of time, resources, or commitment; and thirdly because the results are not properly integrated with planning and purchasing intentions to ensure change. These three sources of failure applied potentially to HNAs in New Zealand in the context of the Health Reforms 2001 and needed monitoring as the research proceeded. Clearly, there would be implications for policy and implementation, should health need assessment be found to be ineffective.

\subsection{Health Reforms 2001 Research}

As indicated in the Preface, the present research formed part of a larger research project, the Health Reforms 2001 Research, the objectives of which were to determine the strengths and weaknesses of alternative ways of organising governance, purchasing and accountability for health care in New Zealand. The research used the opportunity afforded by the reforms that established the DHBs to:

- Document and analyse the new governance, purchasing and accountability arrangements and processes which develop under DHBs.

- Compare the strengths and weaknesses of the DHB model of governance, purchasing and accountability with those of alternative models of organisation.

- Compare the strengths and weaknesses of individual DHBs' approaches to governance, purchasing and accountability.

- Disseminate knowledge about alternative DHB approaches to governance, purchasing, and accountability, and their strengths and weaknesses, to key stakeholders in the sector.

- Develop an improved capability in terms of research experience for research on health care management and organisation in New Zealand in the future. 
(Cumming, Ashton, Barnett, Scott, Kiro, Cunningham, Bolton, Russell, Tenbensel, Powell and Coster, 2001, p.12)

Particular attention was paid to how the DHB model and individual DHB approaches to governance, purchasing and accountability promoted: effective strategic decisionmaking; participation by local communities in decision-making accountability to central government; technical efficiency, equity, and service choice goals; provider accountability to the DHB and the meeting of Treaty of Waitangi obligations.

The research evaluated these reforms against policy goals and the impacts that might be expected from the relevant health policy. The research also drew on the experiences of key stakeholders to identify any unanticipated effects of these reforms. The approach is that suggested by Vedung (1997).

The present thesis research inevitably overlapped with the Health Reforms 2001 Research, and synchronous research methods maintained consistency between them; The researcher took responsibility for the HNA research, within the wider context of the research group.

Care was taken throughout the project to meet the requirements for $\mathrm{PhD}$ research in terms of independence of thought, and the design and conduct of the research. The researcher worked independently on the Health Needs Assessment (HNA) part of the project, but submitted reports to the overall research project.

Based on existing literature, the government's stated goals, and Ministry of Health policy guidelines, this present study is designed to research the process and outcomes related to HNAs, and relate this to the effectiveness of the government's policy settings. The most recent health reforms represented a significant shift away from the purchaserprovider split, although it was retained in the non-hospital sector. The effect of this shift on HNA and planning models will be researched. 


\subsection{Treaty of Waitangi}

By means of explanation of the importance of Māori health need within a framework of health needs assessments, a brief description is first given of the place that Māori people hold within New Zealand. Health need for Māori is addressed in the usual epidemiological terms, but in addition the obligations of the Treaty of Waitangi are considered to apply by both Māori and government. There has been considerable debate recently regarding the rights of Māori to receive preferential access to health services on the basis of ethnicity, rather than health need alone, but at the time of writing there has been no confirmed policy shift by government regarding this matter.

The Māori people of New Zealand are a 'first people' and being indigenous were granted certain rights under the Treaty of Waitangi, signed between Governor Hobson, on behalf of Queen Victoria, and Māori chieftains at Waitangi in the Far North, in 1840. The Treaty of Waitangi is regarded as the founding document of New Zealand. It consists of three Articles, and is translated into both Māori and English versions.

The Treaty of Waitangi Articles provide certain undertakings for Māori people, namely those of partnership, participation, and protection. In 1988 the Royal Commission on Social Policy described those three principles as being of particular importance to health and other social policies as follows:

- Partnership: an on-going relationship between the Crown or its agencies and Iwi. Iwi may be organised in a variety of ways (for example, as trust boards, incorporated societies, runanga) and there may be more than one representative body within a single Iwi.

- Participation: meaningful, positive Māori involvement in all aspects of New Zealand society. This can be at both the individual and group level; and can involve both decision-making and service delivery.

- Active protection: the Crown is to adopt a proactive approach to ensure that Mãori well-being is enhanced wherever possible. In health, active protection is largely about health promotion and preventive strategies. (Royal Commission on Social Policy, 1988)

The significance of the Treaty of Waitangi to health is tied to the meaning of health as a tāonga or treasure for Māori and the use of 'tāonga katoa' in Article Two entitles Māori health to specific protection. 
Despite the acknowledgement by the Crown that the Treaty of Waitangi places special obligations on the Crown in respect to the health of Māori, no reference to the Treaty has appeared in any health legislation, including the Health and Disability Services Act 1993, until recently when it was referred to in the NZPHD Act 2000. Today, there is clear recognition that the Crown has obligations and responsibilities to Māori people to address Māori health need, as a direct consequence of the Treaty of Waitangi, and this remains the case unless there is a policy shift by government.

\subsection{Research objectives}

The key research question for this thesis is:

\section{What was the impact of the HNA policy on District Health Boards?}

It implies the following sub-questions:

1. What kinds of HNAs have been conducted by health authorities in New Zealand, and to what extent have they corresponded with official HNA policy goals?

2. What impact have DHB HNA approaches had on DHB planning processes?

3. Which DHB HNA programmes have been the most effective and least effective, and why?

4. How does the New Zealand experience with HNAs compare with those of other systems and countries?

5. What are the policy implications for health planning, HNA etc? Should the current expectations for, and approach to, HNA be changed, and if so how? 
The aims of this research therefore are:

Part 1. To systematically review the international literature on need, theoretical and disciplinary approaches to HNAs, prioritisation, and the relationship between health services research and policy.

Part 2. To analyse health needs assessments conducted by the Ministry of Health, HFA and its predecessors between 1991 and 2000.

The objectives of this part of the study are to:

(a) Identify and catalogue health needs assessment work undertaken by the Ministry of Health, Public Health Commission and health authorities throughout New Zealand between 1991 and 2000.

(b) Evaluate the impact of health needs assessments on service delivery, decision-making and policy.

(c) Review the overall impact of past health needs assessments (1991-2000).

Part 3. To evaluate health needs assessments and prioritisation undertaken by the DHBs.

The objectives of this part of the study are to:

(a) Evaluate the impact of DHB health needs assessments and prioritisation on health service planning and purchasing.

(b) Evaluate five case study DHBs regarding (a) above.

Part 4. Evaluate the relevance and effectiveness of government policy with respect to health needs assessment, prioritisation, planning and health service purchasing in the context of recent health reforms.

\subsection{Research overview and thesis organisation}

Figure 1 shows a figure presenting the research flow. It was divided into two streams. The first stream commenced with a literature review of HNAs (including discussion of 
the meaning of need) and prioritisation, both internationally and in New Zealand. A typology of HNAs was discussed, and several case studies were used to illustrate HNA and prioritisation. The evolution of HNAs and prioritisation in New Zealand was then considered, particularly for the period from Area Health Boards through to the present time, including the work of the National Advisory Committee on Health and Disability on priority setting. HNAs conducted in New Zealand between 1991 and 2000 were then evaluated by analysing documents, and health managers and researchers were interviewed in depth regarding assessments conducted between 1997 and 2000. Conclusions were then drawn as to the impact of past HNA and prioritisation on health policy and decision-making. The implications for health policy were then considered.

The second stream noted the findings of the literature review, and proceeded to evaluate HNAs conducted by DHBs. The prioritisation frameworks used by different DHBs were examined and compared with each other and with the prioritisation framework of the former Health Funding Authority. The connections between the HNAs, prioritisation frameworks, priorities established by DHBs, District Strategic Plans (DSPs) and District Annual Plans (DAPs) were then researched. Case studies of DHBs that achieved various impacts of HNA on planning and purchasing were used to illustrate possible reasons for the differences. Factors influencing the impact of HNAs on the process from HNAs -> prioritisation $->$ priorities $->$ DSPs $->$ DAPs $->$ budgets and purchasing were noted and implications for health policy discussed. 
Figure 1: Research Organisation

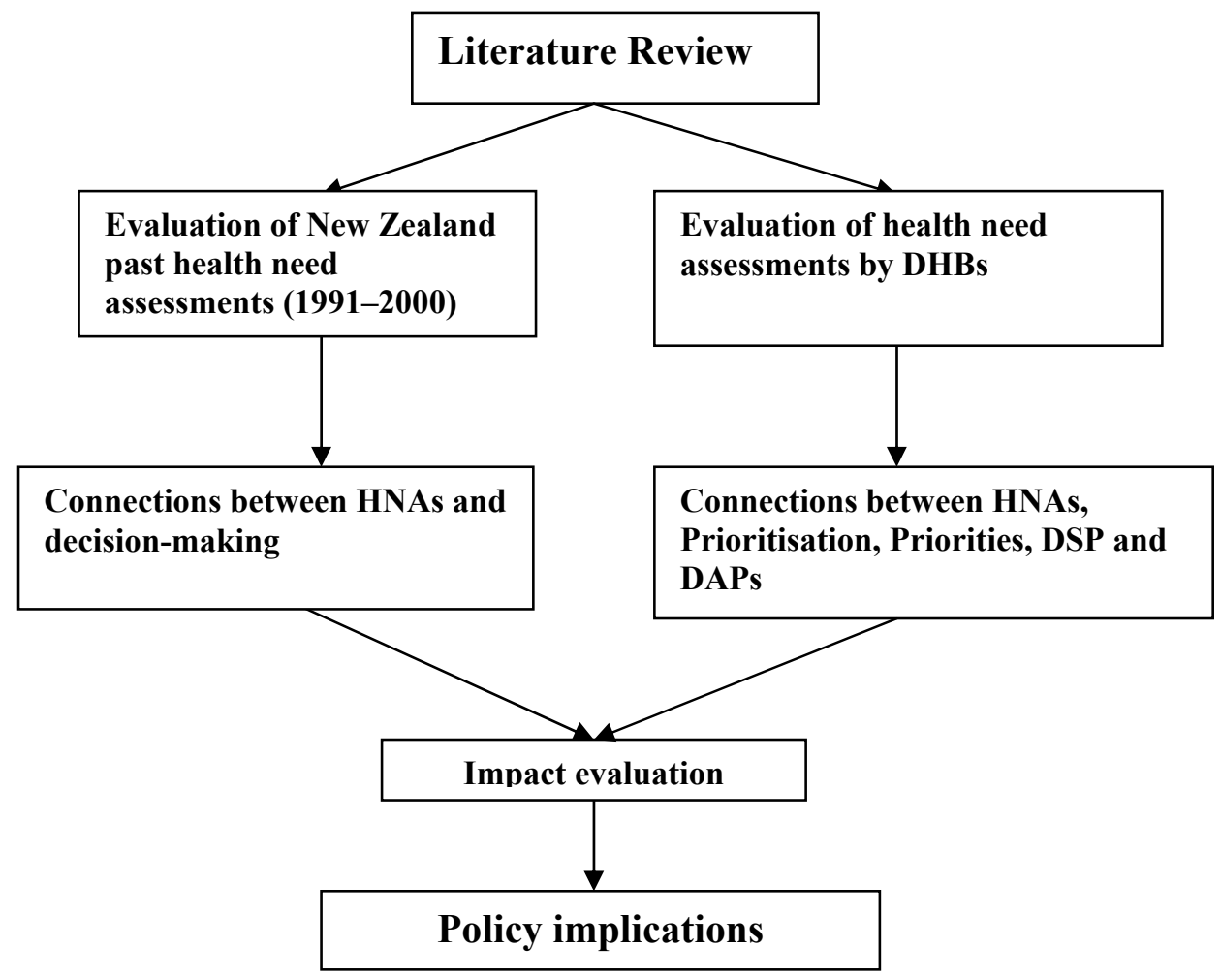

This thesis studies HNA and prioritisation and its impact on planning and purchasing in the public sector in New Zealand. It is not a technical guide on how to conduct HNAs, or prioritise, although it does contain useful background information. The thesis is organised into eight chapters and follows this sequence: Introduction; Literature Review; Typology of HNAs; Health Reforms: Policy Implications for HNAs and Prioritisation; Methods; Past HNAs (1991-2000); DHBs Assessing Health Need; and Conclusion.

Chapter one outlines the background to the research, the Health Reforms 2001 Research, and the objectives of the study. It sets the scene regarding HNA and prioritisation in New Zealand.

Chapter two reviews the literature on HNA and prioritisation. Concepts of need are discussed, as is the relationship between need, demand and supply. The purpose and 
objectives of HNAs are reviewed. The various approaches to prioritisation taken by the National Health Committee and the HFA are considered, along with consultation policies in relationship to prioritisation and planning. The New Zealand and international contexts and experiences are explored.

Chapter three provides a typology of HNAs, including population-based, communitybased, epidemiological, comparative, corporate and economic-based approaches. The various approaches to HNA are described in depth, and four case studies are included to illustrate the application of various types of HNA and prioritisation to planning and purchasing.

Chapter four reviews the various health reforms since the early 1980's and considers the policy implications for HNAs and prioritisation. It describes the influence of four health reforms over the last 22 years, from Area Health Boards to Crown Health Enterprises, Regional Health Authorities, and HFA, until the present time. The current context and policy settings are also discussed.

Chapter five discusses the methods used during the research. It begins with a discussion of evaluation approaches appropriate for this kind of research. This is followed by an explanation of the application of qualitative methods to the current research, including triangulation. The research design and the specific methods employed for each section of the research are described in detail. The four parts to the research are outlined, including Part 1: Literature Review, Part 2: Evaluation of past health needs assessments (1991-2000), Part 3: Evaluation of health needs assessments and prioritisation undertaken by the DHBs, and Part 4: Evaluation of the effectiveness of government policy regarding health needs assessment, prioritisation, and planning. The research timeline is also presented.

Chapter six reports the results of the Part 2 of the research regarding the impact of HNAs on health service delivery and policy during the period 1991-2000. Twenty-five needs assessments during the period 1997-2000 were studied in depth and interviews 
with informants intimately involved in conducting HNAs during those four years are reported. The objectives, processes, outcomes and lessons learnt intimately by those involved in conducting HNAs during those four years are explored. Conclusions are drawn regarding success factors in implementing the conclusions of HNAs.

Chapter seven presents the results of Part 3 of the research considering DHB HNAs: prioritisation frameworks; priorities established by DHBs through District Strategic Plans (DSPs), and District Annual Plans (DAPs); and planning and purchasing. It considers the impact of HNAs on planning and purchasing and the reasons for them. Case studies illustrating different impacts of HNA by DHBs are also presented.

Chapter eight is the conclusion and firstly summarises the key findings from the research. The researcher then discusses the strengths and limitations of the research. The implications for health policy if HNAs and prioritisation are to achieve their stated objectives regarding planning and purchasing are then discussed. Recommendations are made regarding future HNAs and particularly the approach that should be taken to maximise the opportunity to connect HNA and the planning and purchasing processes. Recommendations are also made regarding future research directions. 


\subsection{Introduction}

\subsubsection{Background}

2.1.2 Overview

\subsection{Concepts of need}

2.2.1 Introduction

2.2.2 Need for health care

2.2.3 Interpretation of need

2.2.4 Demand

2.2.5 Supply

2.2.6 Need, demand and supply

2.2.7 Māori health need

2.3 Health care needs assessment

\subsubsection{Introduction}

2.3.2 Purpose and objectives of HNAs

2.3.3 Assessment methods used for HNA

2.3.4 Typology of HNA

2.3.5 Requirements for HNAs to succeed

\subsection{Prioritisation}

\subsubsection{Background}

2.4.2 Core Services Committee

2.4.3 Guidelines

2.4.4 Health Funding Authority prioritisation process

2.4.5 National Health Committee review of the HFA process

2.4.6 PHARMAC's Decision Criteria 
2.4.7 Prioritisation through ring fencing arrangements

\subsubsection{Conclusions}

\subsection{Consultation}

\subsection{Planning models}

2.7 International perspectives regarding the influence of HNA on planning 


\subsection{Introduction}

The literature review traverses the different concepts of need, including historical perspectives; need for health care; demand; the relationship between need, demand and supply; and Māori health need. It then covers health care needs assessment, including purpose and objectives, how health needs are conducted, assessment methods, requirements for HNAs to succeed, and a brief discussion of the typology of HNAs, recognising that this is the subject of a separate chapter (Chapter 3).

In New Zealand, prioritisation is regarded as being closely related to health needs assessment, and was included in the definition of HNA provided by the Ministry of Health for use by DHBs. The various approaches taken to prioritisation by the Core Services Committee, Guidelines Group, Health Funding Authority, PHARMAC, as well as mechanisms used by government to control priorities for health services, such as ring fencing arrangements, are reviewed. This sets out the historical background to prioritisation by DHBs.

Consultation has been an important part of health service planning in New Zealand, with health authorities required to conduct consultation with communities, providers and other interested parties as an integral part of health service purchasing. Various approaches to consultation are discussed. The review provides information relevant to consultation by DHBs, which are required to consult regarding district strategic plans, and any new strategic developments or plans (for example, Māori health plans).

The literature review then describes various models of the health planning process, including comprehensive rational planning, incrementalism, and the mixed scanning model. The chapter concludes with a discussion on international perspectives regarding the influence of HNA on planning.

The public policy, health services research, social science, medicine, public health, general practice and primary health care literature was reviewed for these purposes. 
Searches were conducted using Medline, EMBASE, and PubMed using such key words as need, health care, health need, health care needs assessment, health needs assessment, demand, Māori health need, prioritisation, consultation and health planning. References were obtained from references. Relevant books were searched for references, and Ministry of Health publications for the last ten years were also searched. This resulted in a considerable number of references, many of which are not only used in the literature review, but also elsewhere in the thesis, particularly in the typology, policy context and discussion sections.

\subsubsection{Background}

The New Zealand Public Health and Disability Act 2000 became law in December 2000 and commenced on 1 January 2001. The new Act provides for the establishment of 21 DHBs, each based on the existing geographical areas served by Hospital and Health Services (HHSs). This was done in order to minimise disruption to hospital services and duplication of management roles during the transition from HHSs to DHBs. In addition, there was a desire to allow building from the existing infrastructure and networks, and recognition of existing HHS/community links. With such a goal in mind, the Minister preferred 'an approach that saw DHBs emerging from HHSs' (Minister of Health, 2000c, p.12). Each DHB was now responsible for providing and funding health care services for the population living in its district whereas previously funding for hospitals and community health services was purchased through one centrally based Health Funding Authority (HFA).

One specified responsibility of each DHB under the legislation is to assess the health and disability service needs of its population regularly. This requirement signals an intention to define and respond to the needs of local populations, (Minister of Health, 2000a).

HNA was adopted as a way of prioritising the funding of health care services according to the needs of a community, within available resources. The drive towards health care needs assessment was one part of a package designed to ensure that the public was explicitly involved in determining the type of, and priorities for, health care services 
purchased. Other means included public election of members of DHBs, Board and Committee meetings being open to the public, and public consultation during the strategic planning processes of the DHBs. This included consultation on DHB prioritisation frameworks.

Many countries have been undergoing health care reform over the last decade, including the UK, USA, Australia and New Zealand. Reforms have been driven by the need to control rapid growth in health spending, shifts in the pattern of health service payment and provision, developments in technology, ageing populations, concerns regarding the effectiveness of treatments, and a desire to meet the health needs of populations and individuals better. Health care needs assessment has become an integral part of health planning and policy, by informing the process of health care prioritisation and giving the community a voice in the way that services are provided.

\subsubsection{Overview}

It is necessary to understand the meaning of 'need' and 'health care need' in order to balance the need for health care and the available resources. The literature review explores the concept of need, and explains the relationship between need, demand and supply. It will define need as the 'capacity to benefit' and health care need as the 'capacity to benefit in some way from health care'. An alternative interpretation of 'need' is discussed, but for the purposes of this research and for consistency with the mainstream view indicated by the literature, the more usual interpretation of 'need' will be used.

There are varying definitions of HNA, depending on the interpretation of 'health', 'needs', and 'assessment', who is the beneficiary of such an assessment and to a certain extent how an assessment is conducted. The following section defines health care needs assessment bearing in mind that cost-effectiveness and prioritisation consideration are generally deemed to be relevant considerations. The following definition proposed by myself and adopted by the Ministry of Health will be discussed: 'Health care needs assessment is defined as the assessment of the population's capacity to benefit from 
health care services prioritised according to effectiveness, including cost-effectiveness, and funded within available resources'. (Coster, 2000, Ministry of Health, 2000b)

The various types of HNAs conducted by health authorities will be referred to briefly, but the more detailed description of a typology of HNAs will be held over until the subsequent chapter. The importance of HNA for Māori will be discussed bearing in mind the significant health inequalities that exist for Māori. The importance of choosing a methodology (or range of methodologies) that is appropriate for the purpose and approach of a particular HNA is discussed. The New Zealand context and experience of HNAs will be reviewed, including the Treaty of Waitangi context, particularly with relevance to DHBs.

The literature on the New Zealand approaches taken to prioritisation is reviewed, including those of the Core Services Committee, Health Funding Authority (HFA), National Health Committee and PHARMAC. Health economic approaches including cost-effectiveness, cost-utility analysis, and programme budgeting and marginal analysis are also discussed. The review concludes with discussion regarding community consultation, models of the planning process, and international perspectives on the usefulness and impact of HNAs. There are a number of relevant appendices, including tables of HNAs conducted both in New Zealand and internationally.

\subsection{Concepts of Need}

\subsubsection{Introduction}

This section considers the meaning of need, and the inter-relationships of need, demand and supply. Concepts of need have been described from a diverse range of perspectives, representing the views of sociologists, health economists, epidemiologists and physicians (to name just a few). One perspective commonly referred to is that of Bradshaw. He suggested the widely quoted definition of need as 'capacity to benefit' (Bradshaw, 1972a). Bradshaw proposed a taxonomy of need that recognises four types of need. 
He broadly categorised these as:

normative needs (defined by experts)

expressed needs (needs expressed by action, for example, by visiting a doctor)

comparative needs (comparing one group of people with another)

felt needs (those needs people say they have). (Bradshaw, 1972b)

A normative need for health care arises where at least some 'expert' assessors agree that certain care ought to be provided. This "need" corresponds to what experts want for the community. Experts are accountable to society for their assessments of community need and should be guided by the best research evidence of effectiveness available using 'epidemiological or biostatistical ways of thinking' (Davidoff, Haynes, Sackett and Smith, 1995).

Experts can also be guided by qualitative information, and by experience, particularly where research evidence is incomplete, contradictory or not applicable. Other influences will play a part in considering similar areas or social groups to establish comparative need (Bradshaw, 1972b). Such comparisons can influence experts considering issues of equity in assessment of need. However experts alone cannot define needs.

Expressed needs are signified by requests for care. These may be spoken or expressed in gestures (Metge and Kinloch, 1984) or other actions, such as attendance for care. Expressed needs are typically referred to as 'demands' (Wright, Williams and Wilkinson, 1998a). Demands may or may not be prioritised by those who are expressing them. Where wants are not expressed, other people may infer them. Such inferences should be verifiable with the original source of the want. The greater the likelihood that values and goals vary between people, the greater the need to ask and verify assumptions.

Felt needs refer to what people want or believe they need for themselves or their family members (Carpinter, 1989, Liss, 1993). Liss suggests that these wants differ from needs because to want something implies some knowledge of and desire for it, whereas these conditions do not apply to need. In contrast, Buetow and Coster (2001) contend that 
people's capacity to make choices and have wants depends on how they value things and on their knowledge and 'desires'. Under this interpretation, it is argued that people cannot need something they do not want. The 'neediness' of wants (or goals) further depends upon the potential for harm resulting from failure to achieve them. Society should also help people to have and express informed wants, especially since 'some people are disadvantaged because the needs they report are limited by their awareness of alternatives, low expectations, modesty, deference, or lack of confidence' (Carpinter, 1989).

Considering need according to Bradshaw's structure implies a social administrative view suitable for such public sector services as housing and education. In such areas it is much more difficult to determine with certainty 'capacity to benefit'. But in health care, 'increasing inputs of care can be associated with not only life or death, but also with zero benefit, or negative benefit' (Stevens and Raftery, 1994). In health, increased inputs can be detrimental, such as a procedure undertaken on a patient leading to increased morbidity, rather than improvement in health status.

From another perspective, Buetow and Coster (2001) proposed a theoretical framework for the meaning of health need that is consistent with the New Zealand Health Strategy. In a theoretical discussion we proposed that for individuals or groups to 'need' health care, two sets of criteria must be fulfilled. First, the care must avoid causing 'harm'. Harm in this case is any interference with activities undertaken to meet the 'goals' of the population and its members. Secondly, for individuals or groups to need care, (a) they must have a right to it, (b) they must want it, and (c) some experts must believe they ought to be able to access it. All three conditions must hold for needs to exist because each condition is a necessary but not sufficient condition for need.

\subsubsection{Need for health care}

The underlying assumption of the following discussion is that 'need' refers to the 'capacity to benefit'. Implicit in this definition is the idea that health status may improve when specific services are provided to meet that need. Thus a patient's need for a specific therapeutic procedure is related to his or her potential to derive from it an 
improvement in health status (Sanderson, Hunter, McKee and Black, 1997). While Crampton and Laugesen acknowledge some problems with defining 'need' in this way, they adopt the definition because 'there is no point in devoting resources to health care if there is little chance that people will benefit' (Northern Regional Health Authority, 1995). Similarly Stevens and Gabbay (1991) state that 'there can be no rational need for either an individual, or a population, to receive an item of care that confers no benefit'. Theoretically, if needs are identified, an effective intervention and appropriate resources should be made available to meet those needs.

At this point a distinction should be made between health need and health care need. The term 'health need' acknowledges the wider influences on health status such as housing, education, and employment, and therefore the need for such services in order to improve health status. 'Health care need' is more specific and refers to the need for health care services in order to improve health status.

While the need for health underlies the need for health care, it does not completely determine the need for such care (Gillam and Murray, 1996). The need for health care is much more specific and is closely related to a population's capacity to benefit from health care; it will be dependent on both morbidity and the effectiveness of care. It is the need for health care that is fundamental to health care needs assessments (Stevens and Gabbay, 1991). Assessment of health care needs has become an integral part of health service planning under health service reforms over the past decade. For example, following the 1991 reforms, health authorities in the UK were required to assess the needs of their populations and purchase appropriate health care. Such moves require more effective measurement of the population's health care need than previously so that the question 'Who needs what services - that is, what is the need for health care?' can be answered (Stevens and Raftery, 1994).

It is essential here to differentiate clearly between individual need and population need. Clinicians focus on the individual, with need defined according to what can be done for a particular patient at a particular time, with less consideration of the cost of treatment. There are practical differences between the clinician's view of an individual's capacity 
to benefit and those of the population-based approach taken by epidemiologists and health economists. The clinical view takes no account of those people with health care needs who do not consult a clinician. In contrast, population need must also take account of the people who do not present for care. Costs are viewed differently, as they must be managed if the health of the population is to be maximised. 'Resources are finite, but in a clinical setting, the limits are seldom perceived' (Stevens and Raftery, 1994). Tension between what is best for the individual and what may be best for society will always present a dilemma for clinicians (Gillam and Murray, 1996).

The tension between individual need and population need is comparable to that existing between need, demand and supply. The integration of need with demand and supply is a task that has been undertaken by health economists. However, while demand and supply are concepts inherent to a market paradigm and basic to micro-economics, the concept of need does not fit comfortably within such a framework. The balance between supply of health care and demand has not equated with a population's need for such care (Stevens and Raftery, 1994, Wright, 1998).

\subsubsection{Interpretation of need}

The understanding of need as the capacity to benefit from health care can be interpreted from a range of perspectives, including that of those being cared for and that of those who care. Their respective interpretations will be coloured by their sources of information. As Stevens and Raftery (1994) suggest, a professional's understanding of 'benefits' is likely to be affected not only by 'clinical networks' but also by 'the recent research agenda'. Another factor influencing need is changes in what health problems are prevalent at a particular time. Recent developments that highlight this factor range from new initiatives in the community for the care of psychiatric patients to the much greater role played by women in choosing their obstetric care and the way that it is managed. The interpretation of need can also depend on cultural perspectives on health. Various technological innovations also have an impact on health care needs via the likely benefits resulting from their implementation. The meaning of need is therefore, to a certain extent, contextual. 


\subsubsection{Demand}

Demand can quite simply be defined as what the patient wants; in other words, the health care people ask for. Thus demand is based on self-assessed wants and is what health care providers directly experience when patients request services. Demand can be unlimited. At a primary care level general practitioners function as gate-keepers in controlling demand (Wright, Williams and Wilkinson, 1998b). However, this simplistic explanation of demand belies its complexity and the effects of other factors impinging on it. For example, the availability of particular services may increase the demand for them. Conversely, the unavailability of other services or difficulty of access may result in such services not even being considered, thus reducing demand. One example is long waiting lists, which tend to diminish enthusiasm for surgical procedures in public hospitals and therefore discourages patients from seeking such services.

Important factors that influence demands include geography, demographics of the patient population, individual patient characteristics, characteristics of the health service and health service providers, funding, and political and media influences. Paradoxically, the greatest certainty about demand is its changing nature (Stevens and Gabbay, 1991). The ways demand is met give an indication of the responsiveness and characteristics of health services and the factors that influence supply.

\subsubsection{Supply}

According to Wright et al, society usually permits people to act on 'different views of what needs are ... [though] most doctors will consider needs in terms of health care services they can supply' (Wright, Williams and Wilkinson, 1998a, p.1311). Underpinning this supply perspective is a definition of need as 'a capacity to benefit', where benefit depends on an effective intervention and available resources (Crampton and Laugesen, 1995, Stevens and Gillam, 1998b). Supply, being the provision of health care, is thus dependent on such factors as available resources, political will and prioritisation, interests and availability of health providers and facilities (Stevens and Raftery, 1994, Wright, Williams and Wilkinson, 1998b). From the foregoing discussion it is evident that the elements of need, demand and supply cannot be understood in 
isolation. Considering their interrelationships is a prerequisite to assessment of health needs.

\subsubsection{Need, demand and supply}

The relationships between need, demand and supply are graphically illustrated in the figure below, which illustrates the way these aspects are intertwined but distinct. 'It shows eight fields of services divided into: (1) those for which there is a need but no demand and supply; (2) those for which there is a demand but no need for supply; (3) those for which there is a supply but no need for demand; $(4-7)$ the various degrees of overlap; and (8) the external field where a potential service is not needed, demanded or supplied.' (Stevens and Raftery, 1994).

\section{Figure 2: Interrelationship between need, supply and demand}

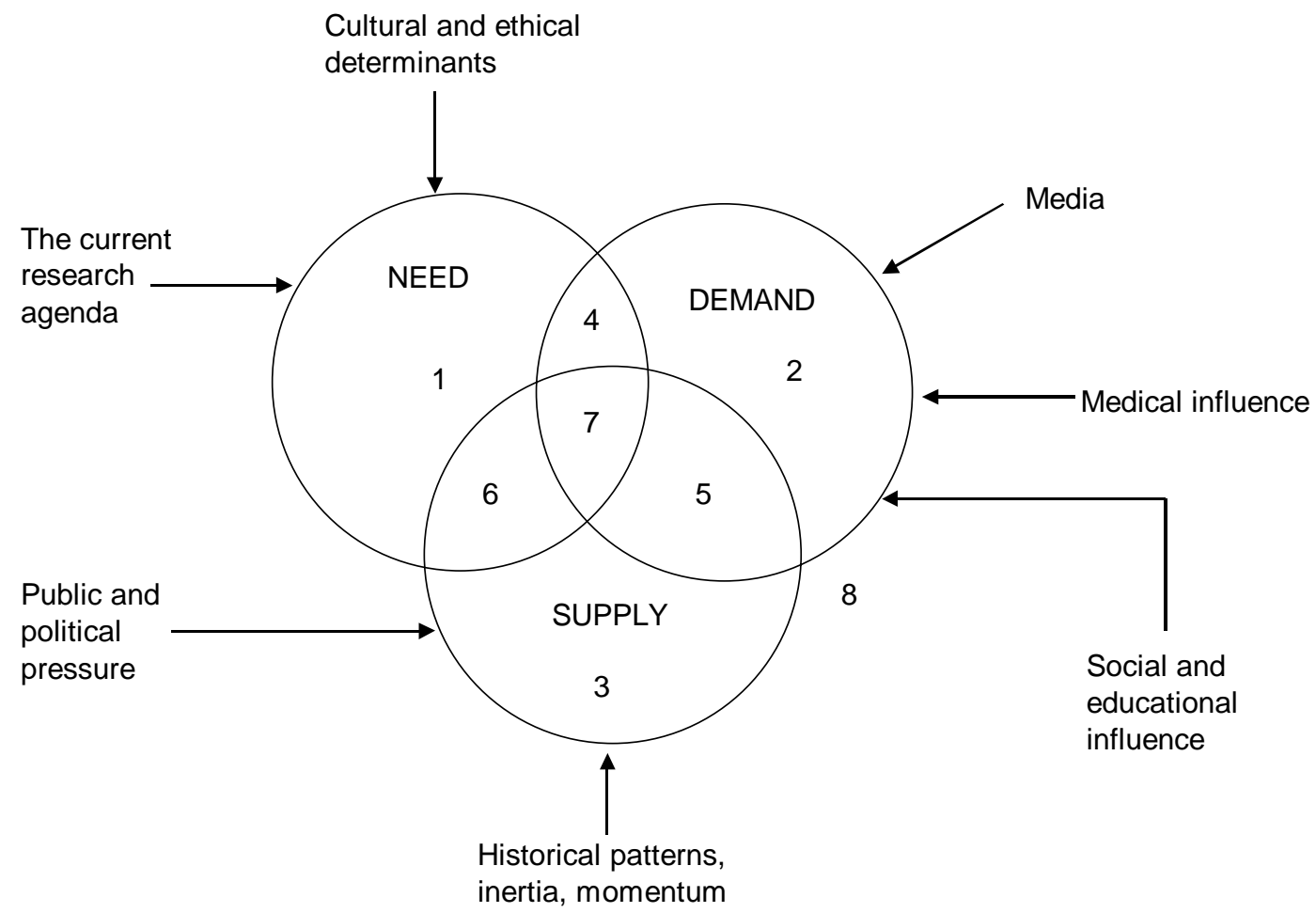

Source: Stevens and Raftery 1994.

Notes: Need $=$ What people benefit from; Demand $=$ What people ask for; Supply $=$ What is provided .

According to Frankel et al (2000), commentators almost inevitably describe as rationing those perceived discrepancies between supply and demand in health care (the curtailment of access to health care). The rationing debate from this point of view is 
described as being far from scientific, and is surrounded by literature that is dominated by assertion, political analysis and ethical debate.

While demand is greater than supply in many cases, it does not inevitably follow that specific health care requirements cannot be met. In fact Frankel (1991) advocates the desirability of 'empirical determination of health-care requirements'. Such an approach requires dispensing with the pessimistic and commonly held view that satisfying demand is destined to futility. He contends that research based on empirical evidence may well reveal that there is 'no need to ration those interventions of undoubted efficacy' (Frankel, Ebrahim and Smith, 2000).

Frankel also asserts that the widely held assumption of an 'inevitable mismatch between supply and demand' is erroneous. Using data from the UK relating to conditions requiring surgical intervention, such as total hip replacement and treatment of cataracts, he observes that such a view has little or no epidemiological support. Common perceptions that factors such as 'increasing life expectancy, new technologies and raised public expectations' responsible for the continuing imbalance between supply and demand in the health-care environment are shown to be inaccurate (p.42). He also argued that:

The demand for health-care must be finite: The population is finite and only a proportion of the population can benefit from and want treatment ... The conventional null, or nihilist, hypothesis that demand always exceeds supply within a public health system reflects neither hope nor experience ... ( $p .43$ )

Referring to the proposed investment in the NHS at that time, he proposed that the programme should be ordered in such a way as to test the hypothesis: "that the limits to demand for key categories of health care lie within the capacity of a properly resourced NHS.' (p.44)

In contrast to Frankel, the New Zealand government has taken the position that health resources are finite, and explicitly stated in the purpose of the NZPHD Act 2000 that the objectives of the new publicly-owned health and disability organisations are 'to be pursued to the extent that they are reasonably achievable within the funding available' (Part 1, section 3 (2)). In other words, that there is an inevitable mismatch between 
demand and supply. Recognising that demand does exceed supply, prioritisation has been deemed necessary and has been provided for in the new legislation. This will be discussed later.

\subsubsection{Māori health need}

Health is regarded by Māori as a tāonga, or treasure, through the Treaty of Waitangi. In addition, Māori perspectives of health and health need are different from those of European perspectives. Furthermore, Māori do not enjoy such good health as nonMāori, and have higher rates of morbidity and mortality. For all these reasons, Māori health need is usually considered separately from the general health needs of the population.

It is widely acknowledged that there are significant gaps in health outcomes between Māori and non-Māori, when taking a population-based approach (Ajwani, Blakely, Robson, Tobias and Bonne, 2003, Ministry of Health, 1999d, Ministry of Health, 1999b, Minister of Health, 2000d, Minister of Health, 2001a, Ministry of Health, 2002a, Ministry of Health, 2002d, Ministry of Health, 2002c). ${ }^{1}$ By means of illustration, this conclusion is simplistically represented in Figure 3 below.

Figure 3: The gap in health outcomes between Māori and non-Māori

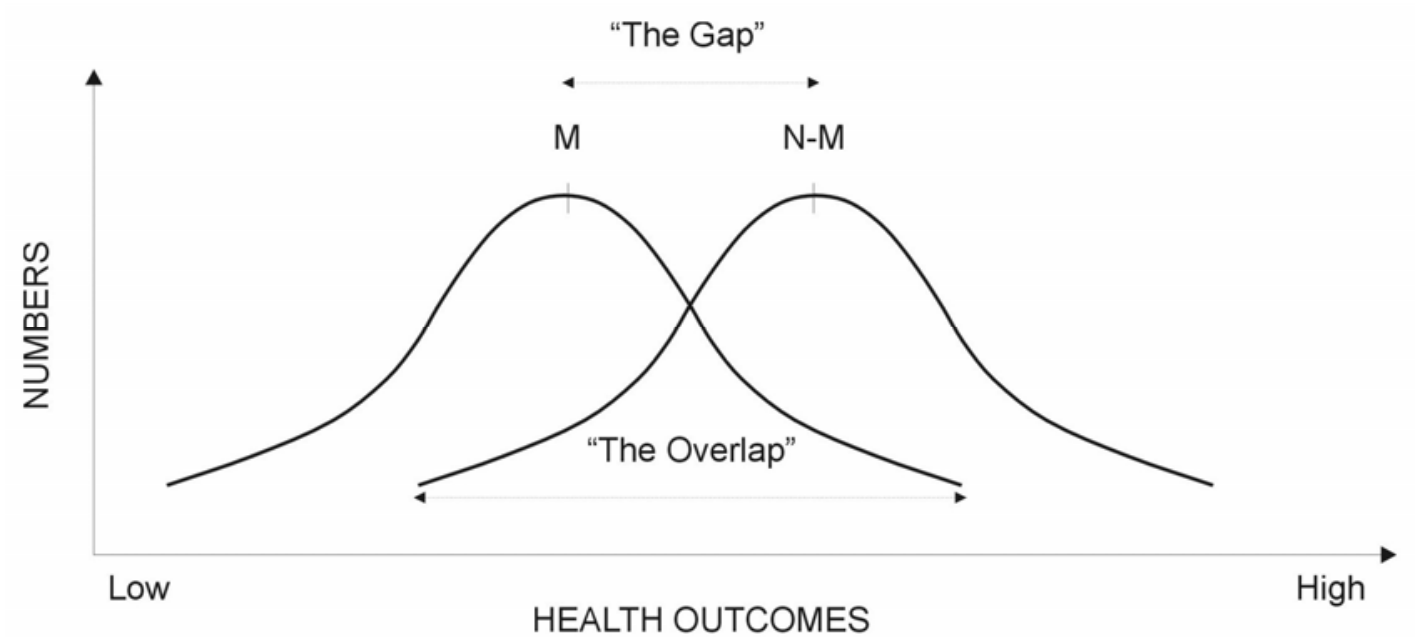

\footnotetext{
${ }^{1}$ See also all Transitional DHB HNAs conducted in 2000 for incoming DHB Boards, 2001.
} 
Some Māori at an individual level enjoy the same level of health as non-Māori, but most do not. Disparities in Māori health status lead to significant Māori health need and consequently entitlement by Māori to equality of health outcomes. The Treaty of Waitangi in Article 3 provides for equal rights for Māori with those of non-Māori, widely interpreted as including the right to good health. ${ }^{2}$ In the past this right of Māori to expect good health has been equated to providing equal access to health services for Māori and non-Māori alike. But for Māori to achieve equality of health outcomes with those of non-Māori, it means that greater access to services by Māori is required in order to address the longstanding inequalities i.e. inequality of access in favour of Māori is required. This has been referred to as a need for vertical equity.

It should also be borne in mind that the perspectives Māori people in regard to health are considered to be different from those of non-Māori. The most frequently cited Māori perspective is that of Durie who proposed a model entitled 'Te Whare Tapa Wha' (the four-sided house) incorporating four health dimensions: taha hinengaro (emotions and mind), taha wairua (spirituality), taha tinana (body) and taha whänau (extended family) (Durie, 1994). These dimensions in the wider context frame Māori health need. This is useful to bear in mind when considering Māori health need and HNA.

There are significant disparities in health status between Māori and non-Māori, and Article 3 of the Treaty of Waitangi provides Māori with the right to equality (including health). The New Zealand Health Strategy, a document whereby the Minister of Health sets out her strategy to improve the health of New Zealanders, as required in the New Zealand Public Health and Disability Act 2000, specifically provides that Māori health outcomes will be addressed and that health inequalities eliminated:

Improvements in Māori health status are critical, given that Māori, on average, have the poorest health status of any group in New Zealand. The Government has acknowledged the importance of prioritising Māori health gain and development by identifying a need to reduce and eventually eliminate health inequalities that negatively affect Māori. (Minister of Health, 2000d, p.18)

\footnotetext{
2 The Treaty of Waitangi is described as the founding document for New Zealand and sets out the rights of Māori in respect of the Crown regarding rights to partnership, participation and protection.
} 
In view of the above, it seems likely that HNAs for Māori will need to:

- Acknowledge the objective of addressing the health disparities between Māori and non-Māori

- Recognise that the goal is equality of health status for Māori and non-Māori

- Assess current Māori health-service delivery

- Define the 'gaps' between Māori and non-Māori health status

- Consider Māori health need in terms of Māori models of health

- $\quad$ Prioritise recognising the existing eight Mãori health-gain priority areas

- Consider the implications for health care delivery

- $\quad$ Consult with Māori regarding health service priorities.

It follows that there are implications for the quantum of various health services that are to be delivered to Māori and non-Māori in the future. Funders of health services will need to consider distributional issues arising from HNAs. There will be implications for health care delivery (unequal needs are likely to be treated unequally, meaning that additional resource may be allocated to meet Māori health disparities). While under the Health and Disability Support Services Act 1993, the Regional Health Authorities and HFA were required to address disparities in Māori health, the New Zealand Public Health and Disability Act 2000 and the related New Zealand Health Strategy are more explicit in this regard, with implications for need assessment including prioritisation, consultation with Māori, and resource allocation for Māori people who are likely to have a greater share of health resources invested in them, compared to non-Māori.

As far as can be determined, there is no other treaty like the Treaty of Waitangi elsewhere in the world, although some treaties cover the rights of indigenous people, but not to the extent of explicit description of the relationship with the Crown regarding rights to participation, partnership and protection. Consequently, it is difficult to draw on comparative literature regarding the rights of Māori to participate in decision-making for resource allocation for health services. The position of Māori people appears to be unique in this regard. 


\subsection{Health care needs assessment}

\subsubsection{Introduction}

Health care needs assessment has its origins in the United Kingdom. The Secretary of State for Social Services proposed that directors of public health should be responsible for assessing the health needs of their populations (Secretary of State for Social Services, 1988). The NHS white paper on "Working for Patients" progressed this idea and reaffirmed the responsibility of the NHS to ascertain the needs of patients, but in order to inform health boards and authorities, as purchasers of care, of those needs (Secretary of State for Health, 1989). The term 'health need' normally refers to the broad environment of individual health and encompasses questions of deprivation and inequality related to the socio-economic determinants of health, as indicated above. Meeting 'health need' is an intersectoral responsibility, and in New Zealand the Government's 'Closing the Gaps' programme intersectorally addresses the gaps in health, education, housing and welfare. ${ }^{3}$

Health care needs assessment should be clearly linked to the concept of need as the 'capacity to benefit', but with the proviso that treatment is considered a reasonable investment. This requirement implies some consideration of the effectiveness and costeffectiveness of the services in which investment is being considered. Because available resources in all health care systems are finite, prioritisation will always be necessary for health service purchasing.

The language defining HNA varies depending on whether prioritisation is recognised as part of the process of HNA. In the UK, Raftery and Stevens referred to three points of the triangle of needs assessment:

3 'Closing the Gaps' was the name given to an interdepartmental government programme to address causes and effects of inequalities, particularly focused on the socio-economic needs of indigenous Māori people with high social need. This programme no longer exists, mainly because 'middle' New Zealand became uncomfortable with the amount of preferential investment proposed to address the 'gap'. 
1. Prevalence and incidence (Public Health Data Set) ${ }^{4}$

2. Effectiveness and cost-effectiveness (Effectiveness Bulletins ${ }^{5}$; Cochrane Centre $^{6}$ )

3. Existing services (Health Service Indicators ${ }^{7}$ ) (Stevens and Raftery, 1994, p.595)

Prioritisation is implicit in this definition because of the mention of cost-effectiveness. The New Zealand Ministry of Health definition of HNA, initially proposed by myself, explicitly includes prioritisation. Although most would regard needs assessment and prioritisation as separate processes, tying them together is a clear signal that needs assessment should not sit apart from prioritisation.

\subsubsection{Purpose and objectives of HNAs}

Determining the purpose and objectives of a needs assessment is an essential first step prior to choosing the approach to be taken for the needs assessment itself. The purpose may be to assess health need of a population; to allocate scarce resources through prioritising; or to assess the requirement for a more extensive study or simply to draw the attention of health planners and funders to problems with health service delivery.

In a review of developing practice in community-based HNA, the London Health Economics Consortium found that among mainly District Health Authorities, that a common problem was failure to state clear objectives, which resulted in the choice of inappropriate or unobtainable objectives by default (Fulop and Hensher, 1997, Hensher and Fulop, 1999, London Health Economics Consortium, 1996). They identified 15 different objectives, which can be grouped into four 'types'.

\footnotetext{
${ }^{4}$ Department of Health (1993n) Public Health Common Data Set. University of Surrey, Surrey. In New Zealand mainly refers to the National Minimum Data Set (NMDS).

${ }^{5}$ Nuffield Institute of Health (University of Leeds), Centre for Health Economics (University of York) and Research Unit of the Royal College of Physicians (1993) Effectiveness of health care (nos. 1 to 7). Leeds: University of Leeds.

${ }^{6}$ Chalmers, I., Dickerson, K. and Chalmers, T.C. (1992) 'Getting to grips with Archie Cochrane's agenda', British Medical Journal, 305: 786.

${ }^{7}$ National Health Service Management Executive (1993) Health Service Indicators. Department of Health, London.
} 
Table 1: Setting objectives for needs assessment exercises

\begin{tabular}{|c|c|}
\hline Type of objective & Examples of type of objective \\
\hline Explicit & $\begin{array}{l}\text { - Obtain community-based view of health needs } \\
\text { - } \quad \text { Aid to future accountability } \\
\text { - } \quad \text { Community input into decisions on health needs } \\
\text { Community input into decisions on health services }\end{array}$ \\
\hline Process & $\begin{array}{l}\text { - Provide evidence to promote health on the agenda of other agencies } \\
\text { - Part of an exercise in education on health issues with communities } \\
\text { - Public relations exercise to assist in raising profile of DHA, or to inform } \\
\text { decisions made by DHA } \\
\text { - Inform wider community-action projects }\end{array}$ \\
\hline $\begin{array}{l}\text { Other unstated } \\
\text { objective }\end{array}$ & $\begin{array}{l}\text { - } \quad \text { Provide general political and local legitimacy } \\
\text { - } \quad \text { Conform to DHA guidance } \\
\text { - }\end{array}$ \\
\hline Hidden agendas & $\begin{array}{l}\text { - } \quad \text { Provision of social commentary } \\
\text { - } \quad \text { Confirmation of particular ideological position } \\
\text { - } \quad \text { Make case for more general redistributive policies }\end{array}$ \\
\hline
\end{tabular}

Source: London Health Economics Consortium 1996 (modified)

Note: DHA $=$ District Health Authority.

In a survey of 217 HNAs in 14 health authorities in London, Hensher and Fulop found that needs assessment directly supported decision-making and action in two-thirds of the studies identified, but that up to 20 percent of needs assessments had no influence on service provision. In 14 percent of needs assessments, the objectives were either not stated or not clear. The authors note that care should be taken to identify those issues that impose a significant disease burden and from which change might result in substantial benefit, and to minimise the impact of high-profile or special interest issues regarding which detailed analysis will add little value (Hensher and Fulop, 1999).

The London Health Economics Consortium (1996) concluded :

Most of the studies we examined failed to state the reasons for undertaking the work or why a particular area was selected or how the exercise fitted in with other planned activities... It was common for aims or objectives not to be explicitly stated in the reports ... It is a truism that the methods are unlikely to be appropriate if the objectives are not clear. (London Health Economics Consortium, 1996, Section 2, p.16)

Few would argue with the fundamental aim of HNAs - to help ensure the provision and supply of equitable delivery of health care. Jordan et al state that clear explanation of the objectives of such assessments is necessary, including how needs assessments can be undertaken, what support will be necessary, and what benefits can follow. The links between assessment processes, prioritisation, and the planning and commissioning of 
local health services need to be understood if they are to produce effective change (Jordan and Wright, 1997).

These points are underlined by Stevens and Gillam (1998b) who, when clarifying objectives and therefore the approach that may be taken to HNA, ask:

- Is the needs assessment about populations or individuals?

- Is there a clear context for allocating scarce resources? (are the needs assessed in the context of priority setting among competing needs?)

- Is the needs assessment exploratory or definitive (is the object to clarify what should be done or just to highlight problems that are accompanied by no obvious intervention)?

- Is the determination of the most important needs based on expert knowledge or participatory methods? (p. 1449)

The answers to these questions guide the choice of approach to HNA that may include population-based, epidemiological, comparative, corporate or cost-effective approaches. All of these methods require enumeration of current services. But in order to meet the objectives other contemporary approaches should be considered including social service assessments; individual healthcare needs assessments; participatory and Oregon-style planning; population and client group surveys; expert specialty recommendations; and clinical effectiveness research.

Yet there are a number of pertinent questions to ask about the objectives of HNA in order to assist decision-making regarding research priorities for needs assessment:

- Is there a realistic chance of achieving change?

- Is the cost of undertaking the work proportional to the likely benefits?

- What are the priorities being suggested by other agencies - the health authority or health board, or social services?

- Does the [purchaser] wish to look at issues that are not directly under their control such as housing and transport? (Wilkinson and Murray, 1998, p.1526) 
A practical problem-based approach is proposed in the following framework of questions to ask when assessing health needs:

- What is the problem?

- What is the size and nature of the problem?

- What are the current services?

- What do patients want?

- What are the most appropriate and effective (clinical and cost) solutions?

- What are the resource implications?

- What are the outcomes to evaluate change and the criteria to audit success? (Wright, Williams and Wilkinson, 1998b, p.8)

\subsubsection{Assessment methods used for HNA}

In reviewing the literature on the importance of the use of the most appropriate methods for conducting HNAs, the researcher fully expected to find more critical commentary than appears to be the case. While it is apparent that the highest standards of research should be applied to the collection of data to assess health need, only a few commentators remark on actual methods appropriate for the various approaches, other than in general terms. It can be reasonably assumed that the highest standards of data collection that apply to research in other areas should be applied to data collection and analysis in HNA.

The following points regarding methods of assessment are summarised from a paper by Murray and Graham who advocate for a coherent, practical and explicit approach in assessing needs for community based, primary, and hospital health services:

- Caution should be exercised when using only a single method of assessment.

- Data may understate the prevalence of disease in a community (similarly the number of inpatient admissions is not a proxy for morbidity in a community in most instances (Payne, Coy, Patterson and Milner, 1994).

- Postal surveys should be interpreted carefully, especially when patients and health professionals may have different understandings of language.

- In-depth interviews should be sufficient in number to achieve meaningful and representative results.

- Results are more likely to be relevant if data are checked against data from other sources (triangulation) to draw conclusions regarding need.

- Different methodologies may be required for the assessment of need to inform the purchasing process (Murray and Graham, 1995, summarised). 
The London Health Economics Consortium concluded from a review of London HNAs conducted in local communities that the process of elucidating the views of the local people remained unclear, and that many of the health authorities and research departments were still experimenting with how to assess local views effectively (London Health Economics Consortium, 1996). They also found that in many cases the methodologies and methods used for HNA were not described in detail. Details of questionnaires, semi-structured interviews, and lists of prompts for focus groups were almost universally omitted, unless the focus of the exercise was on the methodology itself. It was common to list the names of those interviewed, but not the means by which they were chosen, and hence it became difficult to identify any related biases.

Meaningful assessment of needs is stated as the key to successful purchasing (Gillam, 1992). By implication, if the assessment is not meaningful, or accurate, or uses inappropriate methods, then successful purchasing is unlikely to follow. In a study to assess the feasibility of using patients' perceptions of need for primary health care services to develop priorities, Hopton and Dlugolecka (1995b) found that 'Methodological efforts to ensure equal participation in the processes of assessing health needs and of priority setting do not in themselves promote equity.'(p.40) They conclude that some opinions need to be given greater weight in order to promote equity. This means that in HNA, the opinions of minority groups should be normally given greater weight than would otherwise be the case, in order to promote equity. This particularly refers to the voices of least-heard ethnic minorities (e.g., Māori, and other non-Māori ethnic minorities including Korean, Chinese, Indian and Pacific peoples). Assessors will have to work hard to gain the views of minorities and add weight to their views and needs.

Patient surveys can be used to overcome important limitations of assessments of need that are based on the views of health professionals or arise from routine data. Adopting a comparative survey approach was found in some situations to be a useful method for developing an understanding of patterns of need and demand among general practice populations (Hopton and Dlugolecka, 1995a). However, even this method was considered to have limitations and it was felt that the method chosen to collect data in general should be dependent on the information that is required. In some cases it may be 
appropriate to include an in-depth analysis of the needs of particular groups using over sampling methods. This over sampling approach has been found to be useful in the past in order to identify needs that were previously difficult to ascertain (Batterham and Jordan, 1997).

The points made above by various authors show that it is important to choose a methodology (or range of methodologies) that is appropriate for the purpose and approach of a particular HNA. The methods chosen should also be clearly described within HNAs so as to allow proper interpretation of data, and permit repeatability. Different HNA topics will require different methods for the collection of data. These methods may involve a combination of qualitative and quantitative research methods to obtain original information, or the adaptation and transfer of existing information to new HNAs (Wright, 1998).

\subsubsection{Typology of HNA}

It became apparent whilst reviewing the literature regarding HNA that a typology of HNA should be presented as part of this research, based on the various perspectives contained within the literature. Such a typology would allow consistent use of terminology and comparison of the characteristics of HNAs conducted previously. It was also concluded that there was an important place for a description of the various types of HNA within this thesis. Not to do so would leave the reader uncertain as to what was meant by the various terms used to describe types of HNA, and of the approaches used to assess health need. In view of that importance the presentation of the typology has been assigned a chapter of its own, Chapter 3: Typology of HNA.

\subsubsection{Requirements for HNAs to succeed}

There are a number of requirements that need to be met in order for HNAs to be successful. The first relates to developing an understanding of the meaning of need, particularly in relationship to 'health need' that involves intersectoral approaches to the improvement of the health of a community. Secondly, there needs to be an understanding of the purpose of a HNA, of what is involved in assessing health needs, and how that should be undertaken. Few would contest the concept of HNA informing 
equitable provision of health care. But, as pointed out above, a clear description of the objectives of such assessments is required to facilitate a practical understanding of how to undertake and support HNAs. Both health needs assessors, and the public as participants, need to be well informed regarding their respective roles in the HNA process. Thirdly, time, resources and commitment are needed. Those HNAs conducted within short timeframes possibly containing poor quality data may lead to 'wrong' conclusions. In addition, poor timing of the various components of the exercise may also lead to similarly false conclusions, especially if incomplete data are placed before the public during any consultations. An experienced HNA team is necessary in order for methodological rigour to be applied to the collection and interpretation of data collected during a HNA and sufficient resources should be available to the team. Key competencies for health needs assessments include public health and epidemiological knowledge; statistical skills; knowledge of qualitative methods; skills in economic evaluation; consultation skills; local knowledge; and cultural competence. Fourthly, success depends on being able to integrate the results of HNA with planning and purchasing intentions to produce change. Even though a HNA may identify a multitude of needs, which are then prioritised according to the priorities of experts, communities, and purchasers, taking into account health economic approaches, there still needs to be action that follows. Needs assessments need to implemented in order to avoid becoming little more than academic or public relations exercises. Clearly, sound planning processes that incorporate the findings of HNA are required if HNAs are to have any influence on the delivery of health care services (Coster and Buetow, 2002, Jordan, Wright, Wilkinson and Williams, 1996, Jordan and Wright, 1997, London Health Economics Consortium, 1996).

Finally, a useful overview is provided by Wright ed (1998):

The planning cycle should begin with the assessment of need (Womersley and McCauley, 1987). Objectives must be clearly defined and relevant stakeholders or agencies - be they primary care teams, hospital staff, health authorities, the voluntary sector, the media, regional executives, government, or patients - must be involved appropriately. Although such an assessment may produce such a multitude of needs, criteria can be used to prioritise these needs - for example: the importance of a problem in terms of frequency or severity, the evidence of effectiveness of interventions, or the feasibility for change. Needs assessments that do not include sufficient attention to implementation will become little more than academic exercises. (p.8) 
While it is desirable to critique the literature regarding the factors that determine the success or otherwise of HNAs, it can only be observed that there is unanimous agreement between authors writing on the subject, which strengthens my conclusion that a strong consensus position is reached based on their experience.

\subsection{Prioritisation}

\subsubsection{Background}

A brief overview of prioritisation approaches used in New Zealand in the past is presented in order to provide background material for understanding the prioritisation approaches taken by DHBs. This overview of prioritisation commences with the approaches taken by the Core Services Committee, Ministry of Health and Guidelines Group, National Health Committee, and finally the Health Funding Authority, prior to the commencement of DHBs in 2001.

New Zealanders have historically taken a social equity approach regarding access to health and health care services, prioritising on the basis of an underlying set of moral principles rather than a market-driven or economic approach. This, coupled with constant health reform, and linked to indecision regarding centralisation or decentralisation, has suggested that New Zealanders have grappled with the issue of central control and want a moral voice regarding access to publicly funded health services. Generally speaking, this has led to principles-based approaches to prioritisation, sometimes a consensus approach, and occasionally economic approaches including cost-utility, but rarely programme budgeting and marginal analysis.

Principle-based prioritisation is decision-making guided by a set of values or principles held to be important by the society in which the decisions must be made. Attempts to put in place principle-based prioritisation frameworks have been made in Sweden, Holland, USA, Canada and New Zealand (Government Committee on Choices in Health Care, 1992, Honigsbaum, 1991, National Forum on Health, 1997, Swedish Parliamentary Priorities Commission, 1995). 
The HFA had this to say:

The case for a value and principle-based approach to prioritisation or rationing has been made by a number of commentators (Eddy, 1994, Lenaghan, 1997, McKee and Figueras, 1996, Ovretveit, 1997). Two key themes consistently emerge from the deliberations:

1. A principled approach to prioritisation involves moral controversy surrounding the principles themselves.

2. Controversy surrounds where the legitimate locus for making prioritisation decisions should rest.

These themes are evident in the New Zealand experience with prioritisation (Health Funding Authority, 2000d).

\subsubsection{Core Services Committee}

The Oregon exercise in which the public were involved in deciding what services should be publicly funded and accessible for the poor via Medicaid is an early example of a consensus approach to priority setting (Dixon and Welch, 1991, Kitzhaber, 1993, Oregon Health Services Commission, 1991). This has given encouragement to governments, health authorities and others to obtain views from the public on what services should be prioritised and publicly funded (Ham, 1993, Murray, Tapson, Turnbull, McCallum and Little, 1994, NHS Management Executive, 1992). In New Zealand, this task of deciding what should be publicly funded was given to the newly established Core Services Committee in March $1992 .^{8}$

The Core Services Committee was required by its terms of reference to advise the Government on what health and disability support services the Government should purchase so that people could access effective services on fair terms. ${ }^{9}$ It was required to:

- Seek to identify current services in terms of their costs, their efficiency, the range available, their utilisation, and any deficiencies or variations in their provision;

${ }^{8}$ Core Services Committee was the short title given to the National Advisory Committee on Core Health and Disability Support Services established by Hon Simon Upton, Minister of Health, in March 1992, for the purpose of deciding the list of core health services that would be publicly available.

${ }^{9}$ The Government indicated that core services would not necessarily be free and immediate. However, the Government would ensure that any user charges for core services would be affordable, and waiting times reasonable and appropriate. 
- $\quad$ Assess the effectiveness and relative benefits of these services and the potential impact of any recommended changes;

- Consult with the public, and with health professionals and other relevant professionals about the services currently provided and their distribution, and seek views on which services the Government should ensure are purchased and on any desired changes in the distribution of services or their terms of access;

- Recommend annually to the Minister of Health which core health and disability support services should be purchased, how they should be distributed and the terms of access on which they should be available;

- Recommend periodically to the Minister of Health any changes necessary in the future processes for advising the Government on core health and disability support services. (National Advisory Committee on Core Health and Disability Support Services, 1992, p.5)

In effect, the Committee was given the task of conducting a single, national HNA - the task consisted of data collection, needs analysis and prioritisation, all of which are components of health needs analysis.

A significant shift in the Committee's approach to prioritisation became evident in their third annual report where the Committee indicated that it was recommending moving away from identifying key services towards a description of key services that should be publicly funded:

The Committee considers that advice on the kinds and relative priorities of publicly funded services will best be achieved by a description of key services in terms of the circumstances when they will be publicly funded including their terms of access and quality standards (National Advisory Committee on Core Health and Disability Support Services, 1994, p.8). (my bold italics)

The Committee advised that a 'simple list' of core services was not an appropriate way to describe people's eligibility or access to publicly funded disability support and health services. It observed that such an approach did not have the capacity to tailor services to the needs of individuals and communities. It advised that:

Access to publicly funded services should be described in terms of the circumstances in which they should be publicly funded - that is, when they provide a benefit, when they are cost-effective, when they are a fair and wise use of available resources, and when they are in accord with the values of communities.

The Core Services Committee has a work programme of systematic evaluation to identify key services in terms of the circumstances in which they should be publicly funded, that is, to define the boundaries of the core. Not all services need to be defined, nor will they be defined, in this degree of detail (p.7). 
The Committee reported on the specific work programme under way and continued to develop advice on services that it felt would benefit people and the circumstances in which people should have access to publicly funded services. It also reported on work developing evidence on the balance and appropriate mix of services and levels of funding for those services. Among its recommendations were four key principles for use by Regional Health Authorities with regard to purchasing decisions for core services for the1995/96 period. $^{10}$ They principles were that:

- $\quad$ the treatment or service provides benefit

- $\quad$ the treatment or service is value for money

- $\quad$ the treatment or service is a fair use of public funding

- $\quad$ the treatment or service is consistent with the communities'values. (p.8)

In considering the question 'Is it value for money?' the Core Services Committee defined 'value for money' as cost-effectiveness. This required information regarding cost and effectiveness. Fundamental to the effective assessment of health needs was the need to recognise that what could be reasonably expected to be publicly funded was bounded.

\subsubsection{Guidelines}

As the Core Services Committee continued its deliberations, it found that it was unable to define a core of services to which the public would have access. Instead, it signalled a shift to the use of guidelines when in the 1995 Annual Report it stated:

The Committee has consistently argued that the terms under which New Zealanders have access to publicly funded services must be far more sophisticated than a simple list is capable of being. Access must be made clear by describing the circumstances in which the most cost effective services will offer the greatest benefit to individuals. This entails making a judgement firstly that the level of benefit from a particular service is considered worthwhile in terms of the many competing claims on limited resources, and secondly that the people who stand to gain the greatest benefit from services will receive them first.

The Committee advocates the use of evidence-based guidelines as the means of describing the circumstances in which services will be publicly funded. Guidelines

10 Four Regional Health Authorities (RHAs) covered geographical areas for all of New Zealand's population for health purchasing purposes (Northern RHA, Midland RHA, Central RHA, and Southern RHA). These were established under the Health and Disability Services Act 1993. 
are able to add the necessary levels of definition about the circumstances in which a service should be publicly funded that a simple list cannot do. (National Advisory Committee on Core Health and Disability Support Services, 1995, p.7)

By use of the word 'guidelines' the Committee meant 'clear statements of the content and quality of services, and also the terms of access to publicly funded services.'(p.10) The Core Services Committee was now recommending that specifically identified guidelines be the principal vehicle for dialogue and for aligning expectations about publicly funded services. The Committee recommended to the Minister of Health that investment in the development of evidence-based guidelines was needed, and that these would effectively be the basis for prioritisation of access to health care services. The Committee sponsored establishment of the New Zealand Guidelines Committee of which the researcher was a member. Numerous guidelines were produced and as the work expanded the New Zealand Guidelines Group was formed, independent of the Ministry of Health and the National Health Committee. ${ }^{11}$ Guidelines remain today as an important means of establishing access to publicly funded health services.

\subsubsection{Health Funding Authority prioritisation process}

Government during 1997-98 established the Health Funding Authority (HFA) as a single national purchaser replacing the four RHAs referred to earlier, in order to achieve greater national consistency of purchasing of publicly funded health services. In a report to the HFA Board in 1998, the Midland Division of the HFA reaffirmed the necessity for a prioritisation framework which would provide a 'rigorous, explicit, ethical and transparent process based on an agreed set of principles to guide the setting of priorities and the allocation of resources' (Health Funding Authority, 1998d). Among the many recommendations from the Midland report adopted by the new HFA, was a principles-based approach to decision making. They recommended that the HFA should set priorities and allocate resources to fund services that:

1. provide benefit (effectiveness principle)

2. ensure access is fair, by offering equal opportunity for access to groups or individuals who have similar levels of need (equity principle)

\footnotetext{
${ }^{11}$ The National Health Committee (National Advisory Committee on Core Health and Disability Support
} Services) was the new short title given to the Core Services Committee in 1996. 
3. recognise the Crown's objectives for Mãori Health and its obligations under the Treaty (Mäori health principle)

4. are consistent with the needs and values of communities (acceptability principle)

5. are best value for money (efficiency principle). (ibid.)

These were further modified by the Prioritisation Team that formed under the auspices of the Corporate Strategy group of the HFA, resulting in the May 1998 report (Health Funding Authority, 1998b). At that time the HFA Board agreed that in making decisions, determining priorities and allocating resources, it would give regard to the following five decision-making principles:

\section{Effectiveness}

The extent to which health and disability services produce desired outcomes, such as reductions in pain, the maintenance of current activities, the promotion of independence and the prevention of premature death. Services are given higher priority if they produce more of the desired outcomes (where the level of benefit takes into account both the benefit per person and also the number of people benefiting).

\section{Cost}

The total economic costs of services, including flow-on effects, are considered together with the effectiveness of those services, to ensure available funding is used to achieve the maximum possible gain in health and independence status.

\section{Equity}

Equity of outcome was chosen as the main meaning of 'equity' as an operational principle. Equity of outcome is about reducing remediable disparities in health status, for groups with lower levels of health.

\section{Māori health}

In making funding decisions, the HFA acknowledges the Treaty of Waitangi, and encourages Māori participation in providing and using services

\section{Acceptability}

The expectations and values of New Zealanders are taken into account in the HFA's decision-making process.

In order to improve allocative efficiency for publicly funded services, the HFA developed a prioritisation process based on these principles. It centred on an economic framework using cost-utility analysis. However, following consultations with stakeholders and expert groups the proposed process was modified. The revised process consisted of programme budgeting and marginal analysis (PBMA), incorporating economic cost-utility analysis (CUA), and taking into account the prioritisation principles of equity, Māori health, acceptability and other health and disability gain priority areas (Health Funding Authority, 1998b). 
The process was described in further detail by the HFA (Health Funding Authority, 2000d). It has also been described as a process involving seven steps (Figure 4, below) by Ashton et al (2000).

\section{Figure 4: The seven steps in the process}

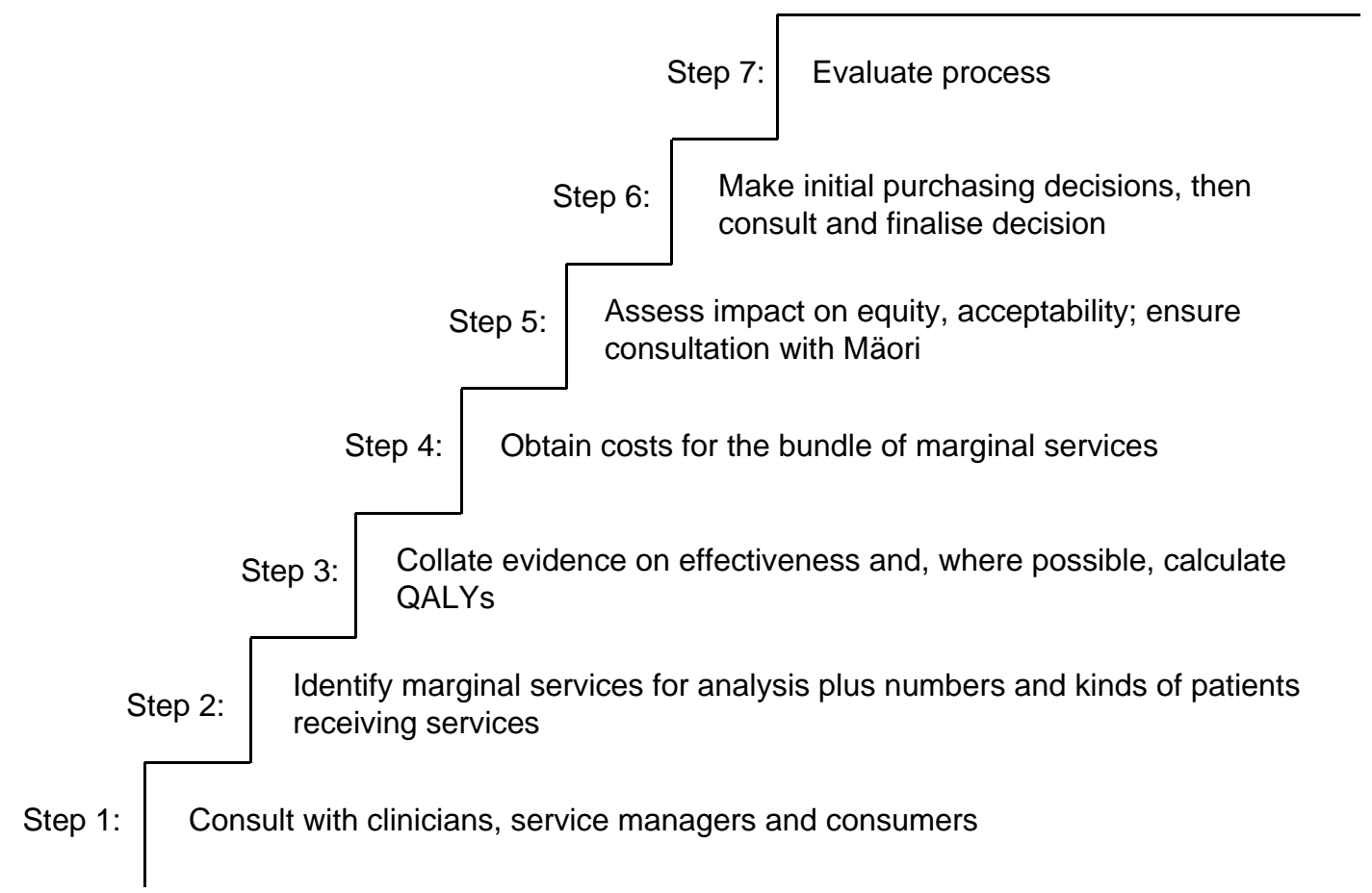

Source: Ashton et al 2000

Note: QALY = quality-adjusted life years. Step 4 also includes flow-on costs and estimating cost effectiveness of programmes.

The final generic process accepted that cost-utility analysis (CUA) could be used as an effective technical tool, but was not the only tool available, and had limitations (as do other economic tools).

\subsubsection{National Health Committee review of the HFA process}

The National Health Committee was concerned regarding the HFA approach to prioritisation and commissioned a review. Ashton et al, in reporting to the National Health Committee on the HFA's prioritisation approach, stated that they were broadly in agreement with the set of principles proposed to determine purchasing decisions. 
However, they noted that:

The key questions in relation to these principles are (i) the means by which each is defined and operationalised and (ii) how the prioritisation process incorporated the principles: the relative weight assigned to each, how trade-offs between principles are to be dealt with, and whether any of the principles provides a veto over the priorities suggested by the others. (Ashton, Cumming and Devlin, 1999, p.19)

The decision by the HFA to use CUA as a starting point for purchasing decisions was arguably a controversial decision according to Ashton. Support for this notion came from Australian research on the opinions of a cross-section of the public, which found little support for the idea that the objective of the health services is to maximise the number of quality-adjusted life-years (QALYs) gained where the consequence is a loss of equity and access to services for older people and for people with a limited potential to improve their health (Nord, Richardson, Street, Kuhse and Singer, 1995). But the concern that remains with Nord et al's research is that respondents simply did not comprehend the concept of opportunity cost (Mooney, 1998). Support for Nord's view came from Sweden where it was argued that cost-effectiveness should be ranked third as a principle for priority setting in health care, and that it should only be applied when comparing methods for treating the same disease (Swedish Parliamentary Priorities Commission, 1995).

It was noted by Ashton that CUA remained in the HFA's August 1998 document as 'the key means by which the principles of effectiveness and cost will be addressed' (Health Funding Authority, 1998c). Ashton recommended that cost-effectiveness act as a 'necessary but not sufficient' condition in decision-making, and that the principles of equity and acceptability should also be addressed. In its full expression, the HFA prioritisation approach involved a CUA combined with analysis of equity, Māori health and independence and acceptability impacts. In a subsequent editorial following the Second International Conference on Priorities in Health Care in October 1998, Klein stated:

Once we acknowledge that setting priorities is inescapably a political process - it involves making painful decisions socially acceptable and mobilising consent among both the health professionals who have to implement them and the public who are affected by them - we can turn to devising the appropriate mechanisms for doing so. (Klein, 1998, p.959) 
With reference to the above statement Ashton commented that:

In this context, we believe that the technical approach proposed by the HFA (Cost Utility Analysis, a form of economic analysis) has merit. We also believe, however, that the important role that values play in assessing priorities and the international experience suggest the need for caution in attempting to apply CUA across the board. In addition, we would argue that the process of setting priorities - including engaging stakeholders - is as important as the technical aspects of CUA. (Ashton, 1999, p.16)

The HFA approach was considered to be ambitious, but Ashton concluded that:

The HFA proposal represents an important step in the right direction towards improving the allocative efficiency of health services. However, it will be important to proceed carefully and explicitly and to pilot the proposed process openly. The process and its costs should also be documented, monitored and evaluated. Outstanding issues that need to be addressed include:

1. Clearer definition of the principles of equity, acceptability and Māori health

2. Clarification of trade-offs between the five principles

3. Development of a clearer framework for identifying marginal services

4. Clarification of which instruments and whose preferences should be used in the estimation of QALYS

5. Assessment of the importance of non-health outcomes that cannot or are not captured by these measures

6. Development of procedures to be taken in cases in which effectiveness, cost and other information are absent or inaccurate

7. Development of some process that ensures that decisions at other levels of the system are consistent with the decision-making principles and practices of the HFA. (Ashton, Cumming and Devlin, 2000, p.174)

The HFA had been piloting the principles-based decision-making framework during 1999 and 2000, including the use of both CUA and PBMA in specific areas. The process of application of the framework and maintenance of the pool of prioritised initiatives is described in careful detail by the HFA (Health Funding Authority, 2000d). In addition, the learning experiences gained as a result of using the framework are also described.

The HFA found the methodology usable for allocation of most new monies, but that it was not universally applicable for all allocation purposes. There were particular concerns regarding prioritisation of disability support services, as the CUA approach apparently did not adequately fully capture QALYs. As a consequence the full 
prioritisation approach has not been used with disability support services. It should be emphasised that the HFA prioritisation process is acknowledged to have limitations but that so far no better system has been devised for use in New Zealand. Early in 2003 the Ministry of Health and DHBs embarked on a process to revise prioritisation principles for use by both for funding of health services. This work is presently still in progress as of December 2003, with public consultation due shortly.

\subsubsection{PHARMAC's Decision Criteria}

PHARMAC is New Zealand's government agency that is responsible for purchasing pharmaceuticals for use in the community, as well as for some hospital pharmaceuticals (from 2002). PHARMAC is of interest because of the approach that it takes to the prioritisation of purchasing, and for the fact that it has survived various health reforms. It was established in 1993 under the Health and Disability Services Act and during this time the cumulative savings made by PHARMAC in the health sector have been estimated at \$NZ2billion, compared to the counterfactual position where no pharmaceutical purchasing intervention were in place.

PHARMAC is a national monopsony purchaser. It has used a set of Operating Policies and Procedures (OPPs) incorporating a prioritisation methodology that is regarded as robust and that has stood the test of time and challenge by drug companies. It is required to 'secure for eligible people in need of pharmaceuticals, the best health outcomes that are reasonably achievable from pharmaceutical treatment and from within the amount of funding provided' (Minister of Health, 2000e). PHARMAC's prioritisation process involves an agreed set of prioritisation principles that includes: the health needs of all eligible people within New Zealand; particular health needs of Māori and Pacific peoples; availability and suitability of existing medicines and therapeutic medical devices; clinical risks and benefits of pharmaceuticals; cost-effectiveness of meeting health needs by funding pharmaceuticals rather than using other publicly funded health and disability support services; budgetary impact; direct cost to health service users; and Government's priorities for health funding (Pharmaceutical Management Agency Ltd, 2000). PHARMAC frequently uses cost-utility analysis and obtains cost / QALY to determine the cost-effectiveness of interventions. PHARMAC's prioritisation process is now regarded as the longest-standing and most advanced prioritisation framework in 
use in the country at the present time. PHARMAC also benchmarks purchasing of therapeutic groups of drugs against those of other countries, including Australia and the UK, and comparisons are made regarding spending on different classes of drugs with new purchasing decisions taking this information into account.

\subsubsection{Prioritisation through ring fencing arrangements}

Ring fencing refers to the process whereby Government determines the amount of funding that shall be made available within the various categories of health expenditure. Current ring fencing arrangements between personal health, mental health services, disability support services, Māori health and public health mean that redistribution of resources between these service areas by DHBs is not allowed. In particular, disability support services were contained within a legally binding governmental resource boundary known as a Non-Departmental Output Class: NDOC, until the recent devolution of disability support funding to DHBs. The use of ring fencing arrangements is effectively a means of prioritisation of services, according to the wishes of Government. Such arrangements remain in place today, and are monitored by Government through the DHB Annual Planning process. However, within each of these health budget areas, allocation of resources according to need is common, using needs analysis and prioritisation.

\subsubsection{Programme budgeting and marginal analysis}

Programme Budgeting and Marginal Analysis (PBMA) is a health economic approach to prioritisation. The combined techniques can be used by purchasers to direct resources so that health care service delivery has a maximum impact on health needs of the local population (Donaldson and Mooney, 1991, Donaldson, Walker and Craig, 1995, Mooney, 1984). The technique of programme budgeting was briefly advocated by Mooney (1977), and was widely used in the 1960's especially in the US by the Federal Government. It subsequently went out of favour until it returned combined with marginal analysis (Donaldson and Mooney, 1991).

The programme budgeting approach describes the current spending pattern of a purchaser, along with where that spending occurs among different groups in the 
population. These groups may be described by disease classification, by disability, or in any other meaningful way (Brambleby, 1995, Davis, Street and Posnett, 1995). However, programme budgeting on its own proved to be of limited value as it is essentially a descriptive technique and is not designed to offer guidance on the way in which current spending patterns could be modified to achieve allocative or technical efficiency. It was not until marginal analysis was added that it became possible to use the programme budgeting framework to explore ways of improving technical efficiency by understanding the cost-effectiveness of inputs (Cohen, 1994, Twaddle and Walker, 1995).

While PBMA has gained some acceptance there are key issues that require careful consideration, such as the time taken for implementation, difficulties in allocating service interventions, reluctance to identify services that may be reduced, difficulties in reaching consensus and priority setting, and difficulty in implementing the results of the process. However, these are not too dissimilar to the limitations of all methods of setting priorities.

Ashton et al. commented on the use of this method by the HFA:

PBMA is still under development as a tool for priority setting in health care. Given the need to spend time on getting participants to understand the process, and given the complex process issues involved, we recommend that the HFA proceed carefully in using PBMA in New Zealand, and consider using it to assist in making decisions within services in the first instance. (Ashton, Cumming and Devlin, 1999, p.5)

The HFA used PBMA in personal health and public health. However, it should be noted that there is little experience with the use of PBMA approaches for disability support services and Māori health services. Prioritisation of these services may well be possible using a PBMA approach, since this is only one step of a principles-based approach recommended by the HFA. For example, PBMA based on child health services would include all possible services, including public health measures.

The system of prioritisation suggested by the HFA supported PBMA, with a principlesbased approach in addition, largely using cost and effectiveness through CUA. Another 
principle was that of acceptability, which was described as "political and social acceptability' (ibid. p.13). This potentially allowed the principle of acceptability to override any other prioritisation process and so effectively gave acceptability the power of veto. If there were to be significant use of PBMA, it appears logical to determine issues of acceptability at the outset, before time and effort are expended on the balance of the prioritisation process. It has been observed that 'the inclusion of acceptability risks becoming a kind of formalised escape clause to avoid contentious decisions, unless it is based upon some clearly articulated and agreed set of precepts regarding justice or human rights' (Ashton, Cumming and Devlin, 1999, p.33). However, the alternative is a contentious set of decisions that would be hard to implement.

Reframing health services for Māori will require consultation regarding Māori health needs. A PBMA approach is quite appropriate, as it is possible to build any level of consultation into PBMA. In order to address issues of equity, the HFA proposed that it would:

... favour services that tend to reduce inequitable disparities in health status, even if those services do not improve overall health status within the larger population as much as some other combination of services might. Thus we are willing to sacrifice some allocative efficiency...in order to reduce inequitable disparities in health. (Health Funding Authority, 1998c, p.23)

The remaining question is one of how such inequities in health status would be prioritised within the framework as a whole. In any event, such a move to equity of outcome is a shift away from the principle of equity of access that has underlined most health policy in New Zealand (Peacock, Devlin and McGee, 1998, Scott, Fougere and Marwick, 1986). Issues to do with the definition of equity arise under such circumstances, along with weighting of outputs (QALYs) for ethnic groups. However, such an approach would be consistent with the Government's policies for reducing health inequalities for Māori (Minister of Health, 2000d, Minister of Health, 2001a). However, a more potent argument is that the issue of equity must essentially relate to equity of health outcomes, and that the issue of equity of access is secondary to achieving that goal. 
Experience with the use of PBMA is reported from overseas (Miller, Parkin, Craig, Lewis and Gerard, 1997, Mooney, Haas, Viney and Cooper, 1997, Peacock, Richardson and Carter, 1997, Ruta, Donaldson and Gilray, 1996); and in New Zealand (Bohmer, 1996, Cumming, Hawkins and Jensen, 1996, p.253-69, McKean, Abernethy, Bobbett, Bohmer, Lock, Paul, Strang and Watt, 1996, Sceats, Hoskins, Moore and O'Dea, 1995, Vaithianathan, 1996). By means of example, Ruta et al (1996) showed how PBMA can be used to formulate a purchasing strategy for child health services. They reviewed policy documents, used traditional epidemiological methods of needs assessment to conduct a health profile for children in Tayside, UK, formulated a programme budget matrix, obtained the views of health professionals and parents, conducted a review of research evidence on effectiveness and cost-effectiveness. The child health strategy working group then reviewed all the available information and prioritised for service development/investment and for service organisation/resource release. They concluded that the approach taken resolved many of the conflicts and difficulties facing purchasers. This example is presented as a case study in Chapter 3.7.4.

\subsubsection{Conclusions}

Prioritisation of health service delivery is a process that systematically, explicitly and transparently defines the use of resources for maximising health gain. The processes undertaken to date have mainly been principles-based and value-driven, and also using methodologies that take account of proven instruments for cost-utility analysis. Consultation with health and disability support providers, Māori and the community, as part of the prioritisation process, will need to be an ongoing feature of resource allocation in the future. Involvement of stakeholders will be important in order to achieve effective analyses of health need and the implementation of the results of the prioritisation process. A range of methods for setting of priorities is available, and further work is ongoing with DHBs and the Ministry of Health to improve prioritisation principles and frameworks.

\subsection{Consultation}

There are linkages between HNA, prioritisation, planning and consultation. DHBs are required to consult with the public regarding prioritisation of services, any change to 
health services, and regarding the DSP. In this section of the literature review the mandate for consultation is considered, consultation principles established by the Health Funding Authority (predecessor to the DHBs) are reviewed, and obligations to consult with Māori are also considered. Methods and approaches to consultation are briefly discussed. Consultation is regarded as one means of obtaining democratic input into decision-making by DHBs.

The mandate for consultation is embodied in the four-fold purpose of the New Zealand Public Health and Disability Act 2000, where it is stated that (among other things) the purpose of the Act is to:

(c) Provide a community voice in matters relating to personal health services, public health services, and disability support services ...

(iii) by providing for consultation on strategic planning.(NZPHD Act 2000, Part 1, Section 3 (c))

The intent of the Government regarding community consultation on health care service delivery is apparent in a Cabinet paper within which the roles of DHBs are defined:

To achieve those objectives District Health Boards will: (a) regularly assess and monitor the health and disability service needs of their populations ... (e) consult and exhibit a sense of social responsibility by having regard to the interests of the communities they serve (Minister of Health, 2000c, p.5).

Experience within the National Health Service in the UK has shown that external input to HNA and the prioritisation of health services is perceived as one way of addressing the 'democratic deficit' in the health service (Jordan, Dowswell, Harrison, Lilford and Mort, 1998). Such perceived democratic deficits within New Zealand are met in part by the election of DHB boards. Further evidence of the significance attributed by the Government to community involvement is contained within a Memorandum to the Cabinet Social Policy and Health Committee from the Minister of Health. It states that one goal of the new arrangements is 'to increase community say over health and disability services' (Minister of Health, 2000f).

The establishment of DHBs, election of the majority of DHB board members, and transfer of funding/functions to those boards is a significant move towards providing a 
community voice in health care needs assessment, prioritisation and service delivery at the district and community level (Crown Company Monitoring Advisory Unit Health Group, 2000, Minister of Health, 2000b, Minister of Health, 2000c, Minister of Health, 2000f). Jordan has identified general requirements for consultation with the general public on needs and priorities, regardless of whether they are current patients or users, or not (Jordan, Dowswell, Harrison, Lilford and Mort, 1998).

In February 2000, the HFA formalised its policies regarding consultation in a document entitled Health Funding Authority Consultation Obligations and Guidelines (Health Funding Authority, 2000b). This New Zealand document sets out the principles applicable to the consultation process, including consideration of the Treaty of Waitangi and other legal obligations, as well as practical issues relating to the process of consultation. The HFA considered that the following principles were of particular importance:

1. Consultation assumes that proposals being consulted upon have not already been finally decided.

2. The HFA will listen to participants with an open mind and value everyone's contribution.

3. The HFA will consult with Māori in accordance with the HFA Mãori Health Policy.

4. Consultation planning, processes and procedures will be publicly explicit, appropriate for the purpose, professionally developed and implemented.

5. All documentation will be honest and easily understood.

6. Consultation will clearly establish parameters and expectations including what has been already decided.

7. All ideas and feedback will be considered when decisions are being made.

8. Consultation will reflect the values of respect and accountability to communities. (Health Funding Authority, 2000b, p.3)

The HFA noted that consultation is a process that is different from notification, negotiation or agreement. It has been defined in law as being more than notification but something less than negotiation and agreement.

Consultation must be allowed sufficient time, and genuine effort must be made. It is to be a reality, not a charade. The concept is grasped most clearly by an approach in principle. To 'consult' is not merely to tell or present. Nor, at the other extreme, is it to agree. Consultation does not necessarily involve negotiation toward an agreement, although the latter not uncommonly can follow, as the tendency in consultation is to seek at least consensus ... 
Consulting involves the statement of a proposal not yet finally decided upon, listening to what others have to say, considering their responses and then deciding what will be done.

Implicit in the concept is a requirement that the party consulted will be (or will be made) adequately informed so as to be able to make intelligent and useful responses. It is also implicit that the party obliged to consult, while quite entitled to have a working plan already in mind, must keep its mind open and be ready to change and even start afresh. Beyond that, there are no universal requirements as to form ... (per J McGechan adopted by the Court of Appeal in Wellington International Airport v Air New Zealand [1993] 1 NZLR 671 at 675).

The HFA also noted that while it may be tempting to view consultation as an exercise to confirm a pre-formed position, the legal view is different. The consultation process consists of setting out a clearly defined proposal, which has not yet been finally decided upon; providing sufficient information about the proposal so that meaningful responses can be made, with adequate time being allowed for preparation of responses; proper evaluation of all responses received, by persons who have not predetermined the outcome; and final decision-making on the proposal.

The New Zealand Health Strategy Discussion Document also cites a number of reasons for ensuring that consumers, communities and providers are involved in strategy or programme development. These include:

- democratic participation: taking into account different perspectives

- partnership and collaboration: fostering shared ownership of solutions to problems, and therefore achieving more co-ordinated, committed action

- equity and fairness: fostering shared understanding, and arriving at equitable solutions

- accountability: from those who design and provide services to those who use them

- acceptability: fostering development of solutions that are acceptable

- $\quad$ ensuring the rights of consumers are upheld

- $\quad$ ensuring provider, community and consumer input is valued

- $\quad$ taking advantage of a range of expertise

- acknowledging and reflecting bicultural values

- adopting a holistic approach: considering issues in relation to communities, consumers and providers, and arriving at practical and effective decisions (Minister of Health, 2000d, p.29).

The process of consultation therefore will involve providers and users of services as well as the community and will allow all parties to have input into major planning 
decisions taken by the Boards. Consultation with the public can be considered from two perspectives. A distinction needs to be made as to whether respondents are provided with information, or whether the respondents are able to engage in any discussion or deliberation in arriving at their own views (Mort, Harrison and Dowswell, 1998). These dimensions define the matrix in Table 2.

Table 2: Approaches to consultation on health care priorities

\begin{tabular}{|l|l|l|}
\hline & Informed & Uninformed \\
\hline Deliberated & $\begin{array}{l}\text { Citizens'juries } \\
\text { User consultation panels } \\
\text { Questionnaire surveys with written } \\
\text { Unformation }\end{array}$ & $\begin{array}{l}\text { Focus groups } \\
\text { Opinion surveys of standing panels/ one- } \\
\text { off questionnaires }\end{array}$ \\
\hline
\end{tabular}

Source: Jordan et al (1998)

\section{Consultation with Māori}

There are some special obligations regarding consultation with Māori. DHBs are required to consult with Māori, including those Māori exercising mana whenua ${ }^{12}$ in order to enable Māori to participate in and contribute to strategies for Māori health improvement (refer NZPHD 2000 ${ }^{13}, \mathrm{Cl} 181$ (c) (Minister of Health, 2000f). The process for consultation with Māori and non-Māori is well described in the HFA consultation obligations and guidelines policy document. The HFA Māori health policy states that:

The Treaty of Waitangi establishes the unique and special relationship between iwi Màori and the Crown. As a Crown agency the HFA considers the Treaty of Waitangi principles of partnership, proactive protection of Māori health interests, co-operation and utmost good faith, to be implicit conditions of the nature in which the internal organisation of the HFA responds to Mãori health issues (Health Funding Authority, 2000b, p.6).

This interpretation of the Treaty of Waitangi obligations in respect to health is the basis of the relationship and the nature of consultation with Māori. Whereas in the past the relationship has been with the HFA on behalf of the Crown, the relationship with the Crown transferred to DHBs at the commencement of the NZPHD Act 2000. The Act

\footnotetext{
${ }^{12}$ Mana Whenua refers to those Māori who are tied culturally to an area by whakapapa (genealogy) and ancestors who lived and died there.

${ }^{13}$ New Zealand Public Health and Disability Act 2000
} 
provides for a special relationship between the DHBs and Māori, requiring DHBs not only to consult with Māori on behalf of the Crown from the outset, but also that DHBs must be cognisant of the impact of new health policies on Māori.

The importance of meaningful consultation with Māori has been emphasised by one local body councillor from the Waitakere City Council in West Auckland. Her contention is that effective community interaction in health will be distinguished by clarity of objectives and roles, inclusiveness, timeliness, innovation in communication, and Treaty of Waitangi consideration with Māori involvement in those discussions. She claims that while "consultation" was a 'buzzword' of the 1980s and 1990s, it has become debased, with the result that 'people have become sceptical that their input will actually make a difference' (Hulse, 1999).

\subsection{Planning Models}

This section is included here mainly because of the relevance of the various models used for planning health services to taking into account the findings of the varying impacts of HNA on planning. During the course of the research, it became apparent that there were differences between DHBs in the way that the planning processes were undertaken, and that this could affect the impact of HNAs on planning and purchasing. The section is not intended to be comprehensive, but rather a description overview of the various planning models used in health service planning.

Early planning thought led to the development of the comprehensive rational model. Simon (1957), beginning with a definition of a decision as a choice between alternatives, states that rational choice involves selecting alternatives which are conducive to the achievement of goals or objectives within organisations. Rational decision-making involves the selection of the alternative that maximized the decisionmaker's values, the selection being made following a comprehensive analysis of alternatives and their consequences. In a critique of Simon's model, Hill (1997) argues that there are four difficulties with the rational approach. Firstly, within an organisation, whose values and objectives are going to be used in the decision-making process? The values of individuals within non-homogeneous organisations may not be the values of 
organisation as a whole. Simon responds to this point by saying that 'a decision is "organisationally" rational if it is oriented to the organisation's goals; it is "personally" rational if it is orientated to the individual's goals' (Simon, 1957, p. 76).

Secondly, it may not make sense to refer to the goals of an organisation. Individuals and groups who often have discretion in interpreting these statements implement general statements of intent within organisations. Goals in public organisations are 'policies', and are likely to be the subject of ongoing modification. Policy is to some extent subject to reformulation as it is implemented, and may be less attributable to the goals of an organisation than the goals of the individuals or groups that make up the organisation.

Thirdly, decision-making rarely proceeds in such a logical, comprehensive and purposive manner. Among the reasons is that it is almost impossible to consider all alternatives during the process of indecision. Knowledge of the consequences of the various alternatives is incomplete. Simon maintains that because of the limits to human rationality that administrative theory is necessary.

Fourthly, regarding organisational rationality, there is difficulty in separating facts and values, and means and ends, in the decision-making process. The ideal rational model postulated the prior specification of ends, and the means by which those ends are reached. Simon's proposed solution is one in which 'The task of decision involves three steps: (1) the listing of all the alternative strategies; (2) the determination of all of the consequences that follow upon each of these strategies; (3) the comparative evaluation of these sets of consequences (p.67).

The means-end model of decision-making is an idealised view of decision-making, and subsequently Simon proposed 'bounded rationality' to recognise decision-making in practice (in Preface to $2^{\text {nd }}$ edn, p.xxiv). Bounded rationality involves the decision-maker choosing an alternative that will not necessarily maximise his or her values, but one which will be satisfactory or good enough. It allows the decision-maker to simplify by not examining all the possibilities, but this may result in important alternatives not being considered. 
Planning using the comprehensive rational planning model takes place in an ordered sequential way, generally involving four tasks. They are:

1. Goal setting: Identification of problems to be solved, needs to be met, opportunities to be seized, and aspirations of stakeholders to be met.

2. Plan formulation: Systematic analysis of alternatives, setting of criteria to choose among the options, examination of consequences of proposed actions.

3. Plan implementation: Deploying a range of actions such as budgets, project schedules and regulatory measures.

4. Monitoring and feedback: Reviewing achievements and updating information, maintaining currency of the plan. (Benveniste, 1989, Parston, 1980)

The comprehensive rational model is described as analytical, information-based and allows system design to be a central concern. It is a set of analytical processes that can be applied to social and political situations, and contexts. It allows normative thinking to be applied to the planning process. The outcomes of the model result from definition of the planning problem, information gathering to formulate the plan, application of governance arrangements to the planning process and the extent to which the planning process is subject to public scrutiny.

This is a somewhat purist model, in that planners seldom start with a blank sheet, as generally there is some activity occurring in the field of interest at the start of the planning process. Neither do planners necessarily have access to full information. Furthermore, top-down activity by expert planners is seldom permissible, as planning tends to be an iterative process, often requiring democratic inputs through consultation. Parallel activities may also be taking place; planning may be occurring in a political environment; and so it may be difficult to follow a chronologically ordered cycle of events. However, essential planning skills needed to cope with complex and changing environments can be built on the steps of the comprehensive rational planning model. The model is criticized for being unrealistic and failing to recognise the inability of planners to collect and process the necessary information in the real world. 
The second distinct model is incrementalism, or what has been described as 'muddling through' (Lindblom, 1959, 1980). It is the antithesis of the rational model. The planning process is not iterative or cyclical, but progression occurs by an incremental approach, moving forwards to the desired objectives. Effectively, this is acting on windows of opportunity as they present, in a series of disjointed steps in changing environments, rather than by a rational approach. It more accurately reflects the real world approach, placing more emphasis on the political dimensions. It allows greater responsiveness but has the disadvantage that existing power structures and relationships are not challenged in the planning process to such a degree. Lindblom argues that incrementalism is both a good description of how policies are actually made, and a model for how decisions should be made. One of the claimed advantages of muddling through is that serious mistakes can be avoided if only incremental changes are made.

The mixed-scanning model lies between the excesses of the comprehensive rational model and the looseness of incrementalism. It assumes bounded rationality: certain key areas are subject to a detailed and structured process of decision-making, while other areas are allowed to evolve unstructured and incrementally. It is pragmatic in approach and less costly in terms of resources, including time and information. The model is attributed to Etizioni who described an approach that rather than considering comprehensively the alternatives for action, focuses only on selected areas of interest. Once the priority areas are chosen, the analytical process hones in on the marginal changes that are possible. The analytical process is otherwise comparable (Etizioni, 1967). In Etzioni's view, fundamental decisions are important because they 'set basic directions' (p.388) and provide the context for incremental decisions. Mixed scanning is an appropriate method for arriving at fundamental decisions because it allows a range of alternatives to be explored. Hill (1997) describes it this way:

Mixed scanning involves the decision-maker undertaking a broad review of the field of decision without engaging in the detailed exploration of options suggested by the rational model. This broad review enables longer-run alternatives to be examined and leads to fundamental decisions (p.106).

Most planning processes occur in the real world where political and democratic influences are at work. Walt refers to the difficulty of bringing together 'macro theories of consensus and conflict and micro theories of decision-making' in the health policy domain. Planning therefore does not occur in isolation from the environment, but in a 
framework with political and democratic inputs mixed with the analytical process (Walt, 1994). Considering that most HNAs are conducted in such a real world, it is hardly surprising that most recent published literature on HNAs seems to relate to either incremental or mixed-scanning approaches to the health planning process.

However, the 1991 NHS reforms placed HNA activity by district health authorities within an explicitly 'rational' framework, as one of the building blocks in a cyclical process of purchasing health care (National Health Service Management Executive, 1991). The Audit Commission (1993) identified three stages: (1) identifying local needs to generate a list of local health needs; (2) rationalising this list to provide a health service 'shopping list'; and (3) prioritising between options to develop a purchasing plan. Ferguson and Ryder (1991) describe the conceptual structure for the health authority purchasing process as one involving a cycle of population characteristics; classification of disease; assessment and statement of health needs; options for service provision; policy statement; contract specification; and monitoring and review. As stated by Hensher and Fulop, 'This framework was unmistakably grounded in the logical/ empirical tradition - a deliberate, formal process of analysis to support optimal decision-making.' (Hensher and Fulop, 1999, p.91).

Although not formally stated, DHBs were similarly mandated to conduct planning and purchasing according to the comprehensive rational planning model. The NZPHD Act 2000 provided for all DHBs to conduct HNAs for their populations; the Ministry required DHBs to prioritise health services according to health need using agreed prioritisation frameworks; DSPs and DAPs were then to reflect prioritised health needs into health service planning; and finally purchasing was to be linked into district annual plans. This planning process is outlined in more detail in Section 4.5.4, p.111. Population-based HNAs in New Zealand therefore fit into the comprehensive rational planning model, but with some exceptions, as will be seen later. 


\subsection{International perspectives regarding the impact of HNA on planning}

The continuous challenge within all health services - to use finite resources to the best advantage of patient care - is resulting in the need to ensure that the delivery of care most effectively meets the health needs of the population (Robins and Rigby, 1995).

The consequence of finite resources is to ensure that those resources are being spent wisely so that the delivery of care meets the health needs of the population. This is in itself a great challenge, as although a HNA may indicate a need for change, implementation of new services often requires disinvestment in some existing services. 'The hardest part of any needs assessment is translating the results into policies and practices that will provide beneficial change' (Wright and Walley, 1998, p.1823). One reason for lack of success is the failure to integrate the results with planning and purchasing intentions to ensure change (Wright, Williams and Wilkinson, 1998b). This is restated by Jordan and Wright, who observed that 'if the results of needs assessments are to lead to changes in services to address the needs identified, then adequate attention must be given to planning and implementation' (Jordan and Wright, 1997, p.696).

Using primary care in the UK as an example, Jordan and Wright conclude that 'current funding arrangements ... not only fail to acknowledge the resource implication, but also make response through service development initiatives difficult' (p.696). HNA will lead to disappointment with publicly funded health services, primary care and communities, if consideration is not given to the implications for new health services. Needs assessment exercises in themselves will inevitably lead to raised expectations of the public for increased service delivery and lack of funding will surely disappoint.

In a study of the impact of community HNAs used in health service planning between 1995-1999 in five regional planning regions in country South Australia, Fuller et al found that needs assessments and regional planning processes had a small impact on the overall allocation of resources for health care services within each region. 
The authors had this to say:

While some needs assessment findings were translated into strategic plans that facilitated additional programs, the most striking observations of the planners was the small impact that the community needs assessments and regional planning processes have had on the overall allocation of resources for health care services within each region. For the time and effort that was involved, the major proportion of a regional health service's budget remains committed to the provision of traditional clinical services with only a small proportion available for the development of new or different services in response to needs. (Fuller, Bentley and Shotton, 2001, p.15)

They also found that HNA was more likely to be effective if it was focused rather than broad-brushed. They concluded that health service reorientation to district plans was invariably a slow process and only time would tell whether HNAs would make a significant impact in the long term. The authors also recommended marginal analysis as a means of prioritising services. Subsequently, in response to this finding, the South Australian Department of Human Services undertook planning based around specific problem areas, rather than taking broad-brushed approaches.

Also bringing an Australian perspective, Hawe argues that HNA should become more change-focused if it is to impact on decision-making (Hawe, 1996). Hawe contends that needs assessment should be directed towards those types of decisions that must be made regarding health service delivery by selectively targeting those approaches that provide information most likely to affect decision-making. 'Data collection procedures should be far more specific and directed than the existing broad-brush procedures that presently serve only a limited purpose and effectively dilute or detract from both agendas for change.' (ibid. p.473).

The Scottish Needs Assessment Programme (SNAP) was established in 1992 as a central, co-ordinated approach to supply some of the required information for needs assessment on a national basis to the NHS in Scotland. In a study to determine how needs assessment was being used to improve health, Hanlon et al (1998) noted three general approaches to purchasing / commissioning taken by health authorities. One third used a 'technical' approach emphasising a formal planning cycle, one quarter emphasised a 'managerialist' dimension including 'identifying clear priorities', and a relational theme was identified by others. The authors concluded that Scottish health 
authorities aspire to a situation where needs assessment drives planning, but recognised that cost and volume issues regarding purchasing predominated.

In a study of HNAs in the NHS, Jordan et al. (2002) defined effectiveness as assessment that resulted in identifiable change in the provision of local health services and / or health policy, using evidence derived from the assessment exercise. They surveyed those involved with 62 HNAs conducted between 1993-8 and found that 45\% of HNAs were effective using this definition. A number of important themes emerged regarding the impact of HNAs on policy and planning. These included careful design, methodological rigour, decisive leadership, good communication, involvement and ownership of the work from relevant stakeholders, support from senior decision-makers, appreciation of the political dynamics and engagement with local priorities, availability of resources, and an element of chance. In addition, they concluded that HNA did not occupy a central position in health service decision-making, remaining vulnerable to a range of factors over which those responsible for its conduct had little or no control. In common with the findings from earlier work, they found that how a topic or issue is selected for a HNA can be crucial to its subsequent capacity to influence action (Fulop and Hensher, 1997). They proposed that this observation supported the 'mixedscanning' approach where needs assessment is used to investigate in detail those areas previously identified as priorities through a wide-ranging scanning approach. Kilduff et al who argued that HNA should start with internally identified areas of concern also supported this (Kilduff, McKeown and Crowther, 1998).

In a review of the impact of HNA on health care decision-making in London health authorities, it was concluded that 'health care needs assessment could be made more effective by striving to improve the issue selection process'. It was proposed that 'intelligent approaches to deliberate scanning of issues... are required to improve the cost-effectiveness of needs assessment activity at a local level.' Furthermore, 'during the "scanning" phase, care must be taken to identify those issues that impose a significant disease burden and from which service change might plausibly achieve substantial benefit.' (Hensher and Fulop, 1999, p.94). The focus in the UK is clearly on scanning the environment for careful selection of issues for HNA activity in order to ensure that it remains cost-effective. 
In the UK, the locus of needs assessment has shifted towards Primary Care Trusts (PCTs) that are now responsible for commissioning (purchasing) services for up to 200,000 people. Formerly HNA was the responsibility of public health, but with the shift towards primary care, HNA has more recently come to be seen as integral to the process by which primary care responds to local and national priorities (NHS Executive, 2003, Wilkin, Gillam and Coleman, 2001). Such HNA activity will need to ensure that it results in effective change according to advice given following earlier studies of needs assessment (Jordan and Wright, 1997).

The literature contains little information regarding the use of HNAs in the USA even though health maintenance organisations must be interested in assessing the health service requirements of their membership. It can only be assumed that this activity is taking place, but that for reasons of market sensitivity that the information does not reach peer reviewed journals. However there is evidence of HNA activity being undertaken in community situations requiring rapid appraisal following emergencies, and examples of this are given in the next chapter. By contrast, New Zealand and Australia, and the UK all have strong planning environments and HNA activity is therefore more evident.

Within New Zealand, democratic philosophies have driven approaches to assessment of health need, and this is now reflected in the requirements for DHBs regarding HNA and prioritisation. But internationally it is particularly uncommon to find HNAs conducted for the whole population of a district or geographical area. Global HNA and prioritisation across all services and needs is rare because it is perceived as hard to do. A review of the literature reveals hundreds of examples of needs assessments done for disease states e.g. (Fletcher and Hirdes, 2001), local service requirements (Toward and Ostwald, 2002, Wells, Klap, Koike and Sherbourne, 2001), disaster planning (Anonymous, 2002), and defined populations (Hanrahan, 2002, Keller and Hedley, 2002), but few for larger area based populations. In Scotland, the Scottish Needs Assessment Project (SNAP) (McEwen, Russell and Stewart, 1995), identified 15 projects, all related to particular health services, not global approaches. They also considered a mix of local and national projects. Key was the recognition that ultimately 
the purpose of HNA was to permit purchasing decisions that result in improved health status.

But not every commentator supports HNA activity, as it is currently undertaken. Referring to health profiles in the NHS, Frankel (1991a) states 'This activity is better seen as a displacement activity that is professionally reassuring at a time of uncertainty, rather than as a productive means of HNA.'(p.257) He notes that research activity has been concerned almost exclusively with the probability of neediness, and not with the distribution of those who might be expected to benefit from particular interventions. In this respect, it is timely to observe more generally that epidemiological data collected on presentation / intervention (treatment) outcomes are skewed by historical artefacts (financial constraints, waiting list juggling, services availability) and therefore do not necessarily represent the community's real requirements for health services, and outcomes i.e. that current use patterns are not necessarily the same as 'needs'.

Williams (1999) argues that burden of disease analyses such as the Global Burden of Disease Study ${ }^{14}$ do not aid decision-making because they do not analyse a question relevant to decision-makers, such as "Should we invest in proposal x or not?" $\mathrm{He}$ suggests that concentrating on 'diseases' as the target of policy interest is mistaken and states that what we need to measure is what impact different interventions will have, not what impact different diseases will have. He states that we do not need to know the global burden of disease, but the marginal impact of health technology on it. Priority setting will be determined by comparison of incremental gains with incremental costs. Williams argues that burden of disease analyses are a waste of scarce resources, other than to identify the scope of the problem for investment or disinvestment. The more

${ }^{14}$ Originally incorporated into the World Development Report 1993, published for the World Bank by Oxford University Press, Oxford, 1993. The main methodological source for the book is Murray, C.J.L. and Lopez, A.D. (Eds.) (1996) The Global Burden of Disease. The Harvard School of Public Health on behalf of the World Health Organisation and the World Bank, distributed by Harvard University Press. Some of the results of the study were published in Lancet $\mathbf{3 4 9}$ in four articles each authored by Murray, C.J.L. and Lopez, A.D., as follows: Mortality by cause for eight regions of the world: Global Burden of Disease Study, 1269-76; Regional patterns of disability-free life expectancy and disability-adjusted life expectancy: Global Burden of Disease Study, 1347-52; Global mortality, disability, and the contribution of risk factors: Global Burden of Disease Study, 1436-42; and Alternative projections of mortality and disability by cause 1990-2020; Global Burden of Disease Study, 1498-1504. 
relevant question is "Would population health outcomes be improved if we invested in $\mathrm{x}$ ?" In short, he argues that what is needed are estimates of the costs and consequences of possible interventions, not estimates of the costs of different service mixes, some of which may be non-feasible. These are the necessary data for value-for-money decisions. Other factors such as effectiveness, equity and binding budget constraint will also have to be considered in prioritisation decisions.

Broad-based population HNAs have also been criticised because they tend to be divorced from the process of programme implementation and do not take account of the difficulties that health authorities face in making changes to programmes. HNAs that are focused on specific population groups, health problems or programmes have been recommended, as these are considered more likely to allow health planners to respond to specifically identified needs (Hawe, 1996, Milewa, 1997, Wright, Williams and Wilkinson, 1998a).

Donaldson and Mooney propose a model for determining priorities within health care that requires no HNA but is based on economic evaluation. They propose that an 'authority' must decide what programmes are priority and that each should then be examined to see whether some reallocation within the programme can produce an overall increase in benefit (using a mixed scanning model and PBMA approach), i.e. the capacity to benefit is the way 'need' is conceptualised, but the focus is just at the margin (Donaldson and Mooney, 1991). This approach used does not take account of equity issues, but does take account of binding budget constraint. HNA data are not necessarily required to establish priority programmes or service areas for examination. Cohen argues a similar economic approach (Cohen, 1994).

Petrou also argues that marginal analysis, rather than needs analysis is more useful for decision-making regarding changes in health service priorities and resource allocation. Potential benefits for health gain are then examined against a range of costed health programmes (Petrou, 1998). 
In summary, international experiences with HNAs, in Australia and UK particularly, demonstrate that they do not occupy a central position in health service decision-making and have only a small impact on planning and purchasing by health authorities. In reality, the major proportion of health service purchasers' budgets is already committed, leaving only a small proportion of funding available for the development of new or different services, according to need. Around the world the trend is for HNA to become more focused in order to fulfil its potential. This approach is therefore closer to mixed scanning rather than the comprehensive rational approach to policy and planning. There is evidence that global population-based approaches are less effective than more focused approaches and it is now uncommon to find HNAs conducted on a whole population of a district or geographical area. Health economic approaches offer another means of determining priorities for healthcare spending using such techniques as PBMA. In addition, the mixed scanning model of planning has generally been found to be more effective than comprehensive planning in bringing change during the planning phase. These trends and conclusions have implications for the use of HNAs in New Zealand. 


\section{Chapter 3: Typology of Health Needs Assessments}

3.1 Population-based approach

3.2 Community-based approach

3.3 Epidemiological-based approach

3.4 Comparative approach

3.5 Corporate approach

3.6 Economic approach

3.7 Case Studies in HNA and prioritisation

3.7.1 Porirua City Health and Disability Report and Plan - example of a population-based approach

3.7.2 Rapid appraisal in an urban setting: an example from the developed world - example of a community-based approach

3.7.3 Renal disease - example of an epidemiologically-based approach

3.7.4 Economics, public health and health care purchasing: the Tayside experience of programme budgeting and marginal analysis - example of an economic approach. 


\section{Introduction}

This chapter presents a typology of HNA for use with this research, based on the various perspectives contained within the literature. It will clarify what is meant by the terms used to describe types of HNA, and the approaches used to assess health need. The typology allows classification of HNAs, and comparisons between the various types, frequencies, characteristics, usages, and resourcing, for research purposes.

According to the Oxford English Dictionary, a typology is 'a classification according to general type, especially in archaeology, psychology or the social sciences. Origin C19; from Greek tupos 'type'.' (Oxford English Dictionary, 2002). Much of health services research falls within the field of social sciences and draws upon its literature. It is therefore quite appropriate to use the word 'typology' to classify various types of HNA.

There are six main types of approach to HNA that form this typology:

1. population-based approach

2. community-based approach

3. epidemiologically-based approach

4. comparative approach

5. corporate approach

6. economic approach

Some authors use a different typology, for instance Stevens and Raftery (1994) who use the typology: epidemiologically based; comparative; and corporate (p.19-20). But they then go on to say that their use of the term 'epidemiological approach' is based on incidence and prevalence on the one hand, and the effectiveness of health care on the other. They note that this method 'combines elements of an epidemiological and health economics approach to needs assessment' (p.19). A similar approach to typology is taken by Stevens and Gilliam (1998a, p.28-31). Rather than combining approaches in this way, for the purposes of this thesis the various types of HNA have been separately listed in order to be clear about their individual characteristics. 
It should be emphasised that in some circumstances several approaches may be used together, and that in others one approach may be used after another. Each of the abovelisted approaches will now be discussed in turn.

\subsection{Population-based approach}

A population-based HNA assesses the health needs of a population using the tools of epidemiology to determine the incidence and prevalence of disease and mortality. Those living in a district generally define such a population, but the population could be defined in other ways including gender, age range, or client group. A population-based HNA will generally go beyond a 'pure' epidemiological approach to needs assessment that only assesses morbidity and mortality data (Ovretveit, 1995, p.66). It may include such additional information as: identified requirements for health services; unmet need; and health service provider information.

A 'pure' epidemiological approach used on its own to generate a HNA results in a 'health profile' of a population, rather than a document describing the wider health needs of a population. Support for this argument comes from Congdon (2001) who states, 'It is increasingly recognised that population health needs assessments based on the comparison of clinical or demographic end points (e.g. mortality rates) neglect population variation in the broader aspects of health status and health-related quality of life.' (p.1). Proxies for need such as area death rates or census indicators are usually imperfect measures of the need for health services since they do not include the full spectrum of morbidity, or provide much information about that part of the population without functional limitation (Erickson, Kendall, Anderson and Kaplan, 1989). Therefore, if population-based HNAs are to be more than just 'health profiles', then they need to go beyond epidemiological data collection and include such additional information as is referred to in the first paragraph above.

\subsection{Community-based approaches}

A community-based HNA is a needs assessment that collects information indicating the needs of a community and lays a foundation for planning for a healthy community. 
Such a community-based HNA will provide information that helps determine: 'the nature and characteristics of a community; whether the current services and initiatives are responding appropriately to illness and are promoting health; where there is a gap in services; where new services are necessary to remove an existing health inequity; what environmental changes are necessary to improve health; how community structures are affecting health and the need for community development.' (South Australian Health Commission: South Australian Community Health Research Unit, 1991, p.8).

Most community-based needs assessments incorporate a high level of user and community involvement and according to Ong et al:

- $\quad$ are concerned with either health services or general social and environmental issues that affect health

- examine small areas or small population groupings

- involve work in the field

- adopt a flexible approach

- $\quad$ base the assessment largely on qualitative data derived from the perspectives of the local community. (Ong, Humphris, Annett and Rifkin, 1991, p.910) (paraphrased)

Community-based HNA should be the key element in planning services that directly or indirectly affect the health of the community, such as recreation, transport, and city planning, recognising the wider social context of health need.

There is debate over whether community needs assessment exercises should be community-based or community-led (Wainwright, 1994). Community-based HNAs are generally led by health authorities, but on the other hand a community-led HNA refers to HNA that is led by a community empowered to provide leadership for needs assessment. This latter model has not been adopted widely, mainly because it is time consuming and appears to be more suitable to the community development models of health care delivery and community action. It may also lead to loss of control by health authorities. However, a small number of health authorities in the UK report the satisfactory use of the community-led needs assessment approach, involving community members as equal partners in the research process (Bromley Health, 1995, Ealing Health Agency, 1995). 
Within the community-based approaches to HNA a number of approaches are used being those of: primary care, community development, and rapid appraisal approaches. Each of these has its own characteristics, which are described below.

\section{Primary care approaches}

Most information on the primary care approach to HNA originates from the UK, where health reforms in 1991 provided for HNA. Some time later, the Secretary of State for Health (1997) required that Primary Care Groups, consisting of a wide range of primary care health professionals, conduct needs assessment based on the populations of their groups (approximately 100,000), using a community-based approach. Numerous authors have described ways of assessing health needs in primary care (Gillam and Murray, 1996, Harris, 1997, Hooper and Longworth, 1997, Murray, Tapson, Turnbull, McCallum and Little, 1994, Murray and Graham, 1995, Murray, 1999, Scottish Needs Assessment Programme, 1998, Shanks, Kheraj and Fish, 1995, Wilkinson and Murray, 1998, Wright, 1998, Wright, Williams and Wilkinson, 1998a).

Primary care approaches to community HNA are also used within communityorientated primary care (COPC). According to Abramson (1988), the essential features of COPC are:

- $\quad$ There must be a defined community or aggregation of people for whose care the service has assumed responsibility.

- $\quad$ Primary clinical care must be provided for individuals in this community. This personal care may be provided by doctors, nurses or other health workers; in different situations it may be curative, preventive or comprehensive.

- $\quad$ There must be defined programmes to deal in a systematic way with the community's major health problems. These community health programmes ... may involve health promotion, primary or secondary prevention, curative, alleviative or rehabilitative care, or any combination of these activities. (p.40)

COPC is an approach that uses epidemiological and clinical skills together to provide programmes for meeting the needs of a population. COPC has been described by numerous authors (Abramson, 1988, Cashman, Fulmer and Staples, 1994, Coster and Gribben, 1999, Crampton, 1999, Freeman, Gillam, Shearin and Pratt, 1997, Garr, Rhyne and Kukulka, 1993, Kark and J H Abramson (eds), 1981, Kark and Kark, 1983, Nevin 
and Gohel, 1996, Nutting, Wood and Conner, 1985, Nutting and Connor, 1986, Tollman, 1991, Wright, 1993).

The actual steps of COPC have been described in the following manner:

First, a primary-care or public-health program defines and characterises the community for which it has assumed responsibility. Second, the program organises and involves the community so that the groundwork for a community-professional partnership is laid. Third, a community diagnosis/needs assessment and a resources inventory are conducted. Fourth, community-based interventions are developed and implemented. And, fifth, ongoing monitoring and evaluation procedures are put in place (Cashman, Fulmer and Staples, 1994, p.54).

These features together constitute a cyclical process beginning with the systematic collection of information, which is then used to implement services followed by evaluation, leading to a continuous feedback of epidemiological and other information.

\section{Community development approach}

The community development model is described as 'a process by which people are involved in collectively defining and taking action on the issues that affect their lives. The process is collective, but the experience is individual. Community development seeks to enable individuals and communities to grow and change according to their own priorities' (Labrynth Training, cited in (Freeman, Gillam, Shearin and Pratt, 1997, Fisher and Gillam, 1999, Fisher, Neve and Heritage, 1999)). Meanings of 'community' have been explored from the perspective of community participation in health promotion (Jewkes and Murcott, 1996). The community development model recognises the social, economic and environmental models of ill health and links user involvement and (purchasing) to improve health and reduce inequalities (Fisher, Neve and Heritage, 1999). The process is different in that community needs assessment is conducted within the community development model as a form of action research. Feedback of data occurs during the various stages of community development. Although the model is regarded as important, it is not common in New Zealand.

\section{Rapid appraisal approach}

The technique of rapid appraisal is commonly used to conduct needs assessments in communities. It has evolved to rapidly assess community need in urban settings, rural 
communities, or following disasters and emergencies (Chambers, 1981, Wood, 1981). Annett and Rifkin (1988) have adapted the framework for health use in low-income urban areas in developing countries. Murray et al (1994) have used the methodology for listening to local voices in health and social planning, while Gillam (1992) discusses its use in obtaining structured views of local communities in general practice situations. It has been used to identify the need for reproductive health care in southern Sudan, using interviews with key informants, in-depth interviews, group discussions, and use of secondary data to involve communities in assessing needs and planning service provision (Palmer, 1999). This study concluded that community leaders and health service providers will not necessarily hold the same view of need as community members, and that rapid appraisal may be a useful tool to identify communities' needs and priorities. The techniques of rapid appraisal are different and involve community consultation as an integral component of the methodology. The objectives are to provide good-quality, timely information, and to include local people, producing results that would lead directly to interventions.

\subsection{Epidemiological-based approach}

'Epidemiologically-based approaches' initially require identification of the population group whose needs are to be assessed (normally a group with a particular disease). Williams and Wright (1998) observe that it tends for the most part to use the 'medical model' of health need, viewing need in terms of the occurrence of specific diseases and health-related states rather than in terms of felt need. Descriptive epidemiology (as opposed to analytical epidemiology - the investigation of the determinants of healthrelated states or events) describes the occurrence of disease in terms of person, place, and time:

1. Person - who the affected people are (in terms of their age, sex, occupation, socioeconomic group etc)

2. Place - where they are when they get diseases and in what way prevalence and incidence vary geographically (locally, regionally, nationally, or internationally)

3. Time - when people get diseases, whether this varies by (for example) season; and how disease occurrence is changing over time. (Williams and Wright, 1998, p.1379) 
The epidemiologically-based approach has been described by Stevens and Raftery and makes a number of assumptions:

1. Needs are best described in terms of disease rather than population groups or services. The logic is that a need arises when there is a reason, rather than when a person has reached a certain age, belongs to a racial sub-group, or because a particular service is provided.

2. Non-local data are valuable for local needs assessment. While significant differences exist in the need for health care services in different localities, often the only source of epidemiology and effectiveness studies is research carried out elsewhere. Prevalence studies undertaken in one locality may apply elsewhere, although local studies, if available, are better. However, cost-effectiveness studies require local calibration, because unit costs are known to vary widely. Generic valuation of health status and estimates of costutility estimates, such as costs per quality-adjusted life year (QALY) gained, may also be specific to the circumstance where the data was (sic) gained, and require careful interpretation.

3. Both costs and cost-effectiveness have to be taken into account in needs assessment. Although need is a function of benefit, not of cost, the purpose of population needs assessment is to help decide between competing priorities; the priorities given to a particular need will depend on both benefits and costs. (Stevens and Raftery, 1997, p.21)

Epidemiologically-based HNAs offer a model of health need assessment based on best available information regarding current health services, prevalence and incidence, and effectiveness and costs to derive optimum models of care based on need, often for particular diseases. The main difference between community-based approaches (see Chapter 3.2, p.70) and epidemiologically-based approaches is that the former are based in the community and are service rather than disease focused, but will utilise the methods of epidemiology, while the latter generally focus on a disease.

\subsection{Comparative approach to needs assessment}

The comparative approach to needs assessment contrasts the services received by a population in one area with those received by another population elsewhere. This approach is particularly useful where there is no definition of an optimum service as it allows comparison of service provision between areas. Comparison of health service delivery between districts can point to differences in the way funding is spent, or can be redistributed, and can highlight the need for additional resources because of recognition of unmet need. Variation in price and service use may be appropriate depending on 
local circumstances, but with per capita funding of health care, gross departures from the mean require justification.

\subsection{Corporate approach to needs assessment}

The corporate approach to needs assessment is based on the demands, wishes and perspectives of interested parties, including political and public views (see Figure 5).

Figure 5: Contributions to the corporate view of local service needs

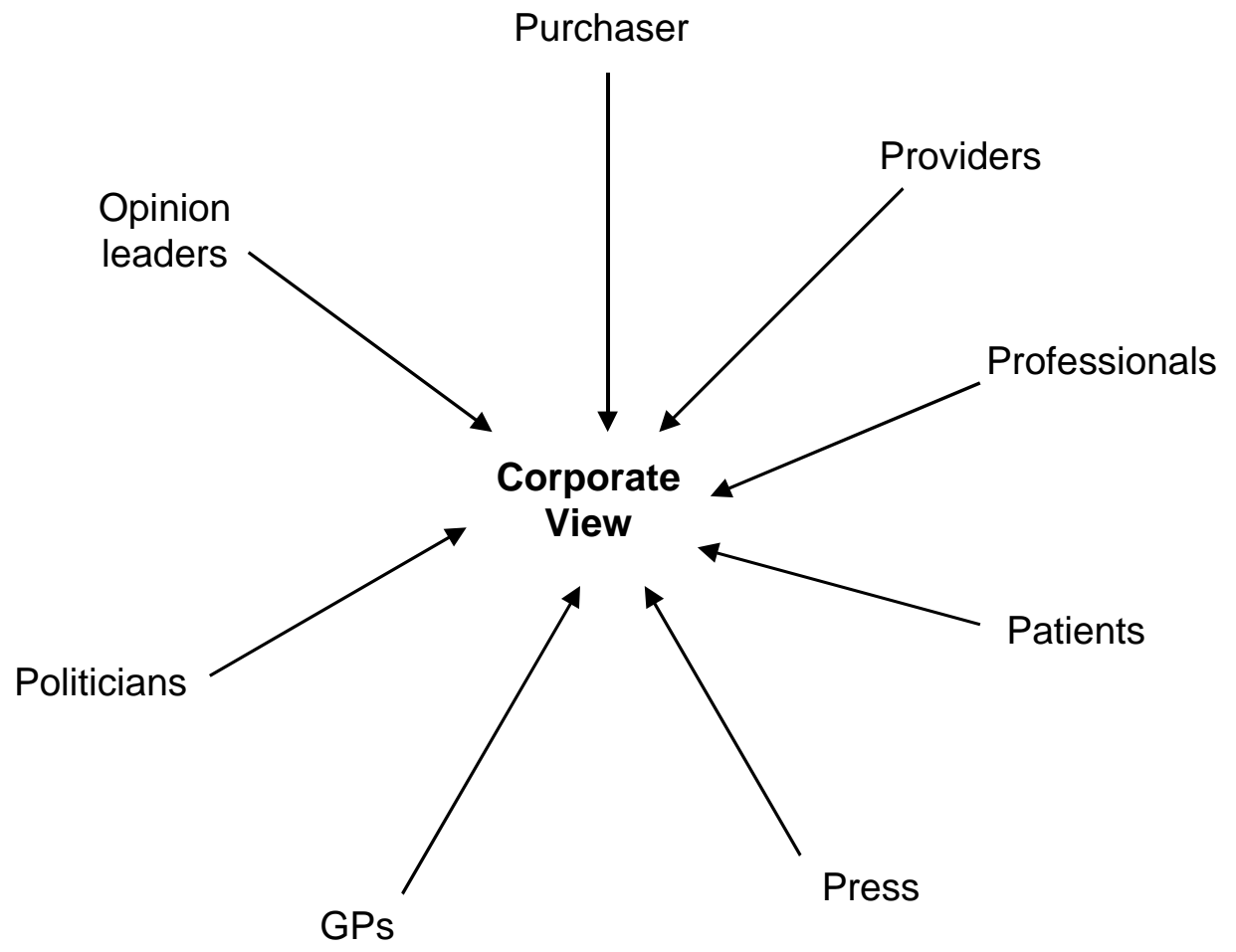

Source: Stevens and Raftery 1997

Corporate approaches involve the collection of data on health services from key informants, including staff of health authorities, provider clinicians, general practitioners and consumers of health services. Whilst there is potential for provider 'capture', failure to consult with clinicians may result in the loss of potentially useful information. Consultation with consumers is important if local knowledge regarding consumer needs is to be obtained. Stevens and Gillam (1998b) observe that where costeffectiveness considerations are weighted equally between services, local people may justifiably attack priorities given to particular services. In addition, while a corporate 
approach blurs the difference between need and demand, and between science and vested interest, it also allows scope for managing supply and demand at the same time as assessing need that is affected by local circumstances (Stevens and Raftery, 1997).

\subsection{Economic approaches to needs assessment}

Although this has been difficult in the past, health authorities are now much more able to quantify the costs of various components of programmes. The economic approach is that health authorities identify 'programmes' that are considered to be relevant in terms of the health authority's strategy regarding disease groups (or government's strategies, or key objectives, or priority areas), where at least the costs of activities contained in each programme are known, or as close to as possible. Secondly, each programme is examined to see whether there are some reallocations within the programme that can produce an overall increase in benefits. In practical terms, a losing subprogramme would be judged to lose less benefit than the gaining subprogramme gained. This process could be extended to all the selected programmes to optimise health gain. This approach does not require large data collections, essentially health profiles, to identify health service requirements or 'need' for health service interventions. Some epidemiological data regarding the population are required, but these are utilised in a service planning or programme approach.

The arguments supporting an economic approach are that resources are scarce and so setting of priorities is necessary. If the aim is to maximise health gain, then in an environment where resource is constrained, some care can be purchased, but some care cannot. Tackling one particular health problem denies society the opportunity to use those resources to tackle another health problem. There are opportunities forgone and therefore opportunity costs. The purpose of priority setting is to ensure that health gains are maximised, and that in the process opportunity costs are minimised. Comparing health care interventions with each other in terms of health gains produced for resources spent can do this. The economic approach addresses two questions: Is an intervention worthwhile, and if so, what is the best way of providing it? (Donaldson and Mooney, 1991, Stevens and Gabbay, 1991). 
Policy questions often relate to the question of purchasing more or less of a programme. The economic question that arises considers the incremental (or marginal) costs and benefits (the difference between costs and benefits before and after the change in scale) of changing the programme. Comparisons can be made using an Incremental Cost Utility Ratio (ICUR) (similar to that used by PHARMAC today) expressed as marginal cost per Quality Adjusted Life Year (QALY). If the objectives of health care policy are to maximise the health of the community within the available resource, then more resources within the health budget should be allocated to treatments with a low marginal cost per QALY, and less resources to those treatments with a high marginal cost per QALY gained.

Health authorities need to achieve allocative efficiency if maximum health gain is to be achieved from available resource. This will not be achieved by a needs assessment process that directs resources to burden of disease profiles, instead of recognising that resources are scarce, ineffective treatments must be discarded in favour of an evidencebased approach (effectiveness) and even if treatments are effective they should be pursued to the extent that costs remain less than benefits and finally that the focus is on changes in service delivery, and the costs and benefits of those changes.

\subsection{Case Studies in HNA and prioritisation}

Four case studies have been chosen to illustrate the connections between HNA and prioritisation, drawing together the sections on typology of HNAs (sections 3.1-3.6) and on prioritisation (section 2.4). Each case study represents a different scenario and approach to heath needs assessment and prioritisation. The first case study is of the Porirua City Health and Disability Report and Plan. This is a population-based needs assessment related to the geographically defined Porirua City. It determines health need, but prioritisation is limited to a choice of two levels. The approach taken is similar to that taken by DHBs, and is probably one of the best examples of population-based HNAs undertaken before the advent of DHBs. One point of difference is that a formal prioritisation framework was not developed, whereas this was required of DHBs. The second case study is that of rapid appraisal in a deprived community in England. Rapid appraisal aims to understand the strength of feeling in a community through identifying 
priority problems. It tries to translate those priorities into action by making a strong link between the community and planners (resource holders) who are capable of instigating organisational changes. The third case study is that of Renal Disease - an epidemiologically-based needs assessment, and shows the approach taken in the UK to assess and prioritise the need for services in chronic renal disease. An economic approach is taken in that cost-effective comparisons are determined by cost per life-year gained, allowing comparison of cost data from various treatment therapies. The fourth case study is an example of an economic model using PBMA. It describes the Tayside experience in HNA and prioritisation of child health services, with the overall aim to maximise quality and length of life for children in Tayside by ensuring effective, costeffective, and equitable use of resources for those services.

\subsubsection{Porirua City Health and Disability Report and Plan - example of a population-based approach}

This is an example of a population-based HNA. It was commissioned by the previous Minister of Health (Hon. Wyatt Creech) to improve health and disability outcomes for the people of Porirua City (Porirua Kapiti Healthlinks Project, 2000b). A similar project was undertaken for the people on the Kapiti Coast (Porirua Kapiti Healthlinks Project, 2000a). The report contains recommendations to the Minister of Health set out in health and disability plans. Importantly, it should be noted that this HNA is based on a local authority (Porirua City), and that it does endeavour to prioritise the list of health service needs that arise.

In 1999 the local authority approached the Minister of Health concerned regarding health and disability outcomes of the people of Porirua City. Funding was obtained and a partnership, known as the Healthlinks Project, was formed between the Council, Māori, Pacific peoples and the community. The Porirua Project Team gathered information about people's health status, health and disability services and their use from a variety of sources. Gathering information on health status and health service use involved collecting and analysing information from the New Zealand Health 
Information Service, Health Benefits Limited, ${ }^{15}$ the HFA, Statistics New Zealand and the Porirua City Council. Additional information was collected directly from service providers, and further national data (often from Ministry of Health publications) were analysed to indicate trends in service use and health risk factors that might apply to Porirua.

Meetings and formal discussions were held with the general public, health and disability providers, community groups, and organisations with an interest in health-related issues. People's views were sought regarding problems with existing services or service gaps, local priorities and how to address these, and from services that were working well. In addition, the Project Team held one-on-one interviews and informal discussions with providers, and also asked providers to complete a questionnaire on service provision and 'gaps' in services. Extensive public consultation was undertaken to ensure that the views of the public, providers and community groups were fully canvassed.

'Hard information' regarding service use, health status and views from the public and providers helped the community and the Healthlinks Project identify what was needed to improve health and disability outcomes in Porirua. Specifically, demographic and epidemiological information was collected on the full range of variables, listed in 56 tables and 20 figures, with data collected in age bands where appropriate. One example of such information is shown in Table 3 below:

Table 3: Numbers and standardised rates of avoidable hospitalisations per 10,000 199498

\begin{tabular}{|c|c|c|c|c|c|c|c|c|}
\hline & \multicolumn{4}{|c|}{ Ambulatory sensitive } & \multicolumn{4}{|c|}{ Preventable hospitalisations } \\
\hline & \multicolumn{2}{|c|}{ Raw } & \multicolumn{2}{|c|}{ Case-mix-adjusted } & \multicolumn{2}{|c|}{ Raw } & \multicolumn{2}{|c|}{ Case-mix-adjusted } \\
\hline & No. & $\begin{array}{r}\text { Rate per } \\
10,000 \text { per } \\
\text { annum }\end{array}$ & No. & $\begin{array}{r}\text { Rate per } \\
10,000 \text { per } \\
\text { annum }\end{array}$ & No. & $\begin{array}{r}\text { Rate per } \\
10,000 \text { per } \\
\text { annum }\end{array}$ & No. & $\begin{array}{r}\text { Rate per } \\
10,000 \text { per } \\
\text { annum }\end{array}$ \\
\hline Porirua & 3,282 & 147 & 2,916 & 139 & 606 & 32 & 900 & 48 \\
\hline Wellington & 20,553 & 117 & 18,236 & 105 & 4,385 & 26 & 6,145 & 41 \\
\hline New Zealand & 211,554 & 117 & 202,538 & 112 & 44,353 & 25 & 76,145 & 42 \\
\hline
\end{tabular}

Source: Porirua Kapiti Healthlinks Project 2000b; based on data supplied by the NZ Health Information Service Notes: Time period covers 1 January 1994 to 31 December 1998. Case-mix-adjusted discharges are based on National AN-DRG, version 3.1, price weights. Standardised discharge rates reflect the average number of discharges per annum over the 5-year period. Rates are calculated using 1996 Census night populations, and use the New Zealand population as the standard. Direct standardisation is used throughout.

${ }^{15}$ Health Benefits Ltd was the government agency responsible for making payments to providers, particularly GPs, and for prescriptions and laboratory testing. 
Not all the material gathered from community and provider discussions was able to be included, but the aim of this participatory research was to reflect the different views held by the many participants. The draft report was circulated within the community and feedback incorporated to the final Report and Plan. The process is regarded as ongoing and it is reported that the community looks forward to implementation of the plan.

Recommendations are made in the areas of health partnerships, equity and fairness (including population-based funding), health promotion and public health services, primary care services, Māori health, Pacific health, maternity, child and youth health, older people's health, dental health, pharmaceutical services, specialist medical and surgical services, disability support services and mental health services.

Recommendations have been discussed and agreed with the organisations involved and in those cases are presented as 'action agreed'. An example from intersectoral action on health is shown in table 4.

Table 4: Actions agreed for intersectoral action on health

\begin{tabular}{|c|c|}
\hline Action agreed & Performance measure (what by when) \\
\hline $\begin{array}{l}\text { Community directs the agenda } \\
\text {-Capital Coast Health as a general principle will } \\
\text { involve local community and voluntary } \\
\text { providers, and key community representatives in } \\
\text { reviewing services and in planning service } \\
\text { changes. } \\
\text { - Regional Public Health of Hutt Valley Health } \\
\text { will offer public health advice and research } \\
\text { support to community groups where the } \\
\text { community projects fit with their strategic } \\
\text { priorities. } \\
\text { - Regional Public Health of Hutt Valley Health } \\
\text { will provide the community with an annual } \\
\text { report to the level of Porirua City, on public } \\
\text { health issues, surveillance data and their } \\
\text { significance. }\end{array}$ & $\begin{array}{l}\text { - Regular service reviews include local providers and } \\
\text { community members in the review team. } \\
\text { - Service planning teams include local providers and } \\
\text { community members in the planning group. } \\
\text {-Epidemiology and research advice for small } \\
\text { projects - } 50 \text { hours/year. } \\
\text { - Advice and support for about two larger projects - } \\
50 \text { hours/year. } \\
\text { - First annual report in December } 2001 \text {. }\end{array}$ \\
\hline
\end{tabular}

Source: Porirua Kapiti Healthlinks Project 2000b

Other recommendations presented for consideration by the Minister of Health were categorised according to actions recommended. An example of this is shown in Table 5. 
Table 5: Actions recommended to Agencies for intersectoral action

\begin{tabular}{|c|c|c|c|}
\hline Action recommended & $\begin{array}{l}\text { What benefits will these } \\
\text { achieve? }\end{array}$ & $\begin{array}{l}\text { Estimated magnitude } \\
\text { of costs }\end{array}$ & $\begin{array}{l}\text { Suggested } \\
\text { priority }\end{array}$ \\
\hline $\begin{array}{l}\text { Intersectoral action to } \\
\text { improve health } \\
\text { It is recommended that: } \\
\text {-Ministry of Health and the } \\
\text { funder support } \\
\text { representatives of Māori } \\
\text { (tangata whenua and } \\
\text { taurahere), Pacific and other } \\
\text { communities in Porirua } \\
\text { (Porirua City Council) to } \\
\text { advise DHB on strategies to } \\
\text { address health disparities, } \\
\text { and facilitate health sector } \\
\text { integration as well as } \\
\text { intersectoral action on the } \\
\text { determinants of health. } \\
\text {-Central government agencies } \\
\text { work with the Porirua } \\
\text { community to address } \\
\text { housing issues for people } \\
\text { with disabilities, including } \\
\text { providing funding for hostel } \\
\text { accommodation with } \\
\text { personal care and home } \\
\text { support. }\end{array}$ & $\begin{array}{l}\text { - Greater impact on health } \\
\text { determinants. } \\
\text {-Programmes more } \\
\text { effective. } \\
\text { - Support for intersectoral } \\
\text { programmes, involving } \\
\text { government, local } \\
\text { authority, iwi and } \\
\text { community to address } \\
\text { the determinants of } \\
\text { health. } \\
\text {-Improving housing for a } \\
\text { small number of people } \\
\text { with disabilities. }\end{array}$ & $\begin{array}{l}\$ 50,000-\$ 100,000 \\
\text { Costs are based on costs } \\
\text { necessary to supplement } \\
\text { existing community } \\
\text { infrastructure. }\end{array}$ & 2 \\
\hline
\end{tabular}

Source: Porirua Kapiti Healthlinks Project 2000b

Evident from the report is the fact that the Healthlinks Project has been well supported by Porirua City Council and its community. Implementation however, will be dependent on the ability of the Ministry of Health to provide funding for this Health Action Zone.

\subsubsection{Rapid appraisal in an urban setting: an example from the developed world - example of a community-based approach}

The following is an example of a rapid appraisal approach to HNA (Ong, Humphris, Annett and Rifkin, 1991). Consistent with the objectives set out by Annett and Rifkin (1988), the South Sefton project took place in Bootle, one of the most deprived wards of the District in the north-west of England. The population of 11,902 comprised 5720 males and 6182 females. The majority of the housing in the district is council owned and a considerable proportion is in need of repair. Because of a high level of unemployment in the area (estimated at 20 percent), there is considerable male outmigration. The percentage of known one-parent families in the area is 6 percent, of whom the majority have children under five. Existing evidence of service use, both 
from health and social services data, indicates that the ward suffers from multiple deprivations.

Researchers within the District Health Authority brought together a research team with the necessary skills to conduct an evaluation of the health and social needs of the South Sefton District using the rapid appraisal approach. Researcher backgrounds included community nursing services, mental health, health promotion, operational planning, clinical psychology, research and development, and from other agencies with a background in housing, family practice, social services and research in social services.

The research team met together at a two-day workshop to refine the objectives and design of the study, and then to prepare the interview schedules for use in the project. There was discussion on how to select the key informants from the community to ensure that the views obtained were representative. Key informants were people who had knowledge of the community because of their profession (for example, social workers, health visitors or police). Within the community, leaders emerged: self-help groups, voluntary associations or other political groupings (for example, play group leaders, chairpersons of elderly groups or councillors). The third category of key informants were people who were centrally placed because of their work or social role within the community. A list of names of people representing all three categories was drawn up. The rationale behind including three distinct sources of information was grounded in the social science approach of 'triangulation', which allows the researcher to look at one issue from various perspectives, and then compare and contrast these in order to reach an inter-subjective account.

The research team was divided into three sub-teams, each containing a representative from the various agencies involved with the research, in order to allow a multisectoral approach to the interviews. Each team was allocated a group of people to interview and the interviews took place over an eight-week period.

Each sub-team identified various themes in a pre-analysis. When the three sub-teams came together these themes were discussed through comparing and contrasting, and 
intersubjective agreement was obtained on the key issues that had emerged from the community.

The subsequent listing of priority problems was as follows:

- physical environment: rubbish, poor housing, air pollution, disposal of syringes, lack of recreational space

- disease and disability: depression and anxiety, drug abuse, chest problems, poor diet

- health services: lack of prevention services for children, too 'busy' GPs, lack of home-care support, lack of well women services, lack of chiropody

- $\quad$ social services: information on social services not readily available, lack of pre-school facilities, fear of the power of social services (to take children away), home helps not free of charge

- $\quad$ socio-economic environment: unemployment, debts, unsafe environment

- valuable resources: strong family support, community action groups. 'Bootle identity', councillors, support from churches, Community Health Council. (Ong, Humphris, Annett and Rifkin, 1991, p.912)

There was considerable difference between the views of professionals and those of the community but the understanding of professionals was mediated by their disciplinary and organisational world view. The research team agreed, however, that the community's prioritisations could not always be taken at face value and that there were issues that needed further debate.

Rapid Appraisal aims to understand the strength of feeling in a community through identifying priority problems. It tries to translate those priorities into action by making a strong link between the community and planners (resource holders) who are capable of instigating organisational changes that will harness community capacity (Ong, Humphris, Annett and Rifkin, 1991).

\subsubsection{Renal Disease - example of an epidemiologically-based approach}

Beech et al (1997) describe an example of an epidemiologically-based approach to HNA. This study, conducted in the context of the National Health Service, addressed critical questions in commissioning services for patients with renal disease. The main emphasis was on purchasing services for patients for end-stage renal failure (ESRF). 
Services required for diagnosis and treatment of acute renal failure and other renal diseases were also discussed. Renal replacement therapy (RRT) is known to be very effective in treating patients with ESRF, who would otherwise die within three to six months. The majority of patients achieve an acceptable quality of life, but complex treatment has to be given regularly following transplantation and the overall cost is high, for what is a relatively small number of people. The renal disease needs assessment considered purchasing options for districts, by describing what was currently known about the epidemiology of renal disease, the need for renal services, and the effectiveness and cost-effectiveness of these services. Further areas for research were identified.

The report began with a statement and context of the problem (renal diseases without failure, renal failure, acute renal failure, chronic renal failure, ESRF) and identified the associated ICD9 codes. Various subcategories were identified. Prevalence and incidence were reported for each of the conditions and were related to the ICD9 data for discharges per 10,000 population. Rates of acceptance on to RRT were noted from the European Dialysis and Transplant Association Register. Age-specific incidence rates for ESRF were also noted, together with ethnic rates of RRT.

Services available for prevention, diagnoses and treatment of renal disease across a range of settings were noted by disease entity. The number of new patients accepted for RRT was recorded by region for the UK, alongside dialysis units, stock of patients accepted for RRT and modalities by which they were maintained prior to transplantation. The annual cost to the National Health Service of maintaining a patient on RRT was also described (hospital haemodialysis, home dialysis, Continuous Ambulatory Peritoneal Dialysis [CAPD], kidney transplant operation and maintenance).

The effectiveness of services for chronic renal failure was described in terms of preventive actions that physicians could take to manage predisposing diseases (for example, hypertension, diabetes, urinary tract infection, urinary obstruction and acute renal failure). The effectiveness of services for diagnosing and treating renal disease was reviewed, including survival rates following transplantation. With regard to cost- 
effectiveness of treatment of ESRF, RRT is expensive relative to other health interventions. Cost-effectiveness comparisons were determined by cost per life-year gained, allowing comparison of data from various treatment therapies. In all, the studies indicated that the main methods for treatment, in order of cost-effectiveness were:

- successful transplantation

- $\quad C A P D$ (home haemodialysis depending on case-mix and circumstances)

- home haemodialysis

- hospital haemodialysis.

Modelling of population need was done using the London Implementation Group's Report on Renal Services to construct a flow diagram to anticipate future need in the steady state. This had implications for the future configuration of services, particularly transplantation. Prioritisations, outcomes and targets for transplantation and funding of organ donation were determined. Recommendations for monitoring and evaluation of renal services, alongside the future research agenda, were also proposed. This epidemiological review of renal disease, particularly regarding ESRF, models the requirements for future renal services on an evidence base. This particular epidemiological review forms part of a set of epidemiological reviews conducted in the UK (Stevens and Raftery, 1997).

\subsubsection{Economics, public health and health care purchasing: the Tayside experience of programme budgeting and marginal analysis - example of an economic approach}

This section describes an example of programme budgeting and marginal analysis and reports the Tayside experience (Ruta, Donaldson and Gilray, 1996). The exercise was conducted to consider purchasing priorities in child health in Tayside for 10 years, commencing in 1996. It was decided that a PBMA approach would be taken in order to release resources from various programmes and make new investments in child health, consistent with national policies. In Tayside, a small working group was formed to oversee the project, consisting of a consultant in public health medicine, area nurse, medical advisor and assistant director of finance. Over a six-month period a strategy was developed that clearly identified priorities for purchasing in child health. 
Policy documents on the provision of child health services were reviewed, including national policy documents published by government and professional bodies, local policy statements and strategies produced by the Health Board and Regional Council. The main outcome of the review of policy documents was to establish a very clear set of goals for the pattern of provision of child health services up to the year 2005. These are summarised in Box 1.

Box 1: Goals for the pattern of provision of child health services in Tayside by 2005

\section{Overall aim:}

- To maximise quality and length of life for children in Tayside by ensuring effective, cost-effective, and equitable use of resources for child health services.

Goals:

- To move towards primary care as the principal focus for health and health care for children at Tayside.

- To increase the proportion of child health care directed towards prevention and health promotion.

- To care for children outside hospital whenever possible and to minimise the length of any hospital stays that are unavoidable.

- To ensure a child- and family-centred service with children, siblings and their parents or carers experiencing a 'seamless web' of care, treatment and support, as they move through the constituent parts of the NHS and related local authority services.

These goals will be achieved by pursuing the following objectives:

- To shift from hospital-based secondary care to integrated or shared care between hospital and primary care, with the emphasis on management by the primary care team using clinical guidelines.

- To increase provision of services by paediatric community nurses offering specialist care in a primary care setting, particularly for patients with chronic illness such as asthma and diabetes, and for children with physical and learning disability.

- To expand the range of services provided in the community by professions allied to medicine.

- To shift away from hospital inpatient care to day-case surgery, outpatient care, day care and 'hospital at home' care.

- To move towards an increasingly specialised secondary care hospital and community service.

- To commission jointly a range of integrated services which meet both health and social care needs of children in Tayside.

Source: Ruta et al 1996

Epidemiological methods of needs assessment were used to compose a health profile for children in Tayside with epidemiological and demographic information collated with 10-year population projections. Health and social services activity data (for example, hospital admissions, outpatient attendances, children registered with a disability) provided valuable 'proxy' indicators of morbidity. The findings revealed that four conditions - asthma, tonsillitis, limb fractures and otitis media - accounted for almost 40 percent of the total admissions for children under the age of 15. 
The majority of health care for children was found to be provided in the community, with 260,000-280,000 general practitioner consultations occurring per annum for children in the 0-14 years age group. Respiratory, ear, nose and throat and gastrointestinal consultations accounted for 64 percent of all consultations.

\section{Programme budget matrix}

A programme budget matrix was formed, with settings broadly categorised into secondary/ tertiary and primary care, and then further subdivided. Programmes are listed across the top of the page; data on expenditure and activity were obtained from datasets within the Health Board contract management system, which included both financial and hospital discharge information. These data were entered into a table and gives a pattern of expenditure within the child health strategy programme matrix (see Table 6, p.90). As can be anticipated, a number of estimations needed to be made:

- A pilot study in two general practices was used to determine that 30 percent of GP consultations were with children, and it was assumed that expenditure on children would also approximate to 30 percent of the budget.

- Data were accessed from the Tayside Medicines Monitoring Unit to identify that 5 percent of the pharmaceutical budget related to children.

- Consultations with GPs were shown to relate to children in 30 percent of cases.

- Similarly, children use 20 percent of ophthalmic services.

- Local NHS providers provided information regarding community services expenditure related to community child health and community nursing services.

The programme budget helped to define a very disparate service. The very process of designing and constructing the programme budget raised issues about possible ways of changing service provision for children. It was noted that the disadvantage, at this stage, was the time taken to construct the programme budget.

The views of health professionals in Tayside were obtained through a two-stage process. Firstly, a multi-professional advisory group was formed with representation from general practice, community paediatrics, child psychiatry, clinical psychology, hospital and community nursing, physiotherapy and social work. This group was given the remit to suggest areas for service development with substantial potential for health 
gain and to identify areas where the reduction or reorganisation of a service might release resources for reinvestment, with a net result of achieving greater health gain for the resources spent. Group members were provided with a summary of the policy review, the results of the need assessment and the programme budget matrix. Each member was asked to suggest up to 10 areas for service development and 10 areas for reorganisation; they were asked to specify the nature of any proposed change, and to justify their choice in terms of health gain.

The second stage involved a postal survey of health professionals working with children. Respondents were asked to consider the epidemiological information, programme budget matrix and again to choose 10 areas for new investment and 10 areas for service redevelopment. Questionnaires and information packs were sent to 262 professionals across Tayside and a 50 percent response rate was obtained. Table 7 shows the top 10 areas suggested for service development in each case. 
Table 6: Programme budget matrix for the Child Health Strategy in Tayside

\begin{tabular}{|c|c|c|c|c|c|c|c|c|c|c|c|}
\hline & \multicolumn{11}{|c|}{ Expenditure (£000) } \\
\hline & $\begin{array}{l}\text { General } \\
\text { paediatrics }\end{array}$ & $\begin{array}{l}\text { Surgical } \\
\text { paediatrics }\end{array}$ & $\begin{array}{l}\text { Special care } \\
\text { baby unit } \\
\text { (SCBU) }\end{array}$ & $\begin{array}{l}\text { Child } \\
\text { psychiatry }\end{array}$ & $\begin{array}{l}\text { Mental } \\
\text { handicap }\end{array}$ & $\begin{array}{l}\text { Community } \\
\text { paediatrics }\end{array}$ & Dental & Ophthalmology & $\begin{array}{l}\text { Health } \\
\text { promotion }\end{array}$ & Other & Total \\
\hline \multicolumn{12}{|c|}{$\begin{array}{l}\text { Secondary and } \\
\text { tertiary care }\end{array}$} \\
\hline Expenditure & 3888 & 2497 & 2338 & 387 & 263 & & 58 & 131 & & 0 & 9562 \\
\hline Activity & 3779 & 2707 & 600 & 11 & 89 & & 138 & 170 & & 0 & 7494 \\
\hline \multicolumn{12}{|l|}{ Out-patient } \\
\hline Expenditure & 445 & 529 & & 327 & 0 & 898 & 1010 & 121 & & 0 & 3330 \\
\hline Activity & 2491 & 5747 & & 269 & 0 & Pop. $(71,943)$ & 247 & 868 & & 0 & 9622 \\
\hline \multicolumn{12}{|c|}{ Day-case/day-patient } \\
\hline Expenditure & 6 & 137 & & 0 & 433 & & 107 & 8 & & 0 & 691 \\
\hline Activity & 26 & 581 & & 0 & 4533 & & 348 & 33 & & 0 & 5521 \\
\hline \multicolumn{12}{|c|}{ Primary care } \\
\hline \multicolumn{12}{|c|}{$\begin{array}{l}\text { Services provided by } \\
\text { community trusts }\end{array}$} \\
\hline Expenditure & & & & 71 & 131 & 2594 & 671 & & & 0 & 3467 \\
\hline Activity & & & & 1630 & 2103 & Pop. $(71,943)$ & Pop. $(71,943)$ & & & 0 & 3733 \\
\hline \multicolumn{12}{|c|}{$\begin{array}{l}\text { Pharmaceutical } \\
\text { services }\end{array}$} \\
\hline Expenditure & 1613 & & & & & & & & & 0 & 1613 \\
\hline Activity & Pop. $(71,943)$ & & & & & & & & & 0 & Pop. $(71,943)$ \\
\hline Expenditure & 0 & 0 & 0 & 0 & 0 & 0 & 0 & 0 & 250 & 0 & 250 \\
\hline Activity & 0 & 0 & 0 & 0 & 0 & 0 & 0 & 0 & Pop. $0-25$ years & 0 & 0 \\
\hline \multicolumn{12}{|l|}{ Total } \\
\hline Expenditure & 11,963 & 3163 & 2338 & 785 & 827 & 4396 & 5356 & 522 & 250 & 0 & 29,600 \\
\hline Activity & 6296 & 9035 & 600 & 1910 & 6725 & Pop. $(71,943)$ & 733 & 1071 & 0 & 0 & 26,370 \\
\hline
\end{tabular}

Source: Ruta et al 1996, p.188. Note: Community paediatrics includes child health surveillance/immunisation/screenings 
Table 7: Top 10 suggested areas for service development and resource release, ranked by frequency of mention

\begin{tabular}{|c|l|l|l|l|l|}
\hline Rank & Service development area & Score & Rank & Resource release area & 1323 \\
\hline 1 & Children with special needs & 866 & 1 & School health services & 568 \\
\hline 2 & Community liaison nurses & 702 & 2 & Health visitors & 527 \\
\hline 3 & Respite care & 653 & 3 & Child development centre & 459 \\
\hline 4 & Child protection & 456 & 4 & Child protection & 433 \\
\hline 5 & Physiotherapy & 421 & 5 & Respite care & 419 \\
\hline 6 & Services for adolescents & 416 & 6 & Dundee Royal Infirmary, Ninewells \\
\hline 7 & Day patient care & 404 & 7 & Hospital bed numbers & 312 \\
\hline 8 & Health visitors & Child development centre & 268 & 9 & Management \\
\hline 10 & School health service & 222 & 10 & Developmental screening & 323 \\
\hline
\end{tabular}

Source: Ruta et al 1996

The views of parents as consumers were regarded as important, and to identify those views a small in-depth focus group of parents of children with different health problems was established. Secondly, a free-phone telephone line was set up for a 24-day period with advertisements in the local paper inviting parents to phone in.

A literature review was undertaken in four service areas (treatment of otitis media, respite care, role of the health visitor, and role of the school nurse). These areas were selected because they were identified as possible candidates for service development or resource release by parents and providers, although there was some disagreement among providers over their perceived cost-effectiveness. Evidence was obtained for the best approaches to managing these services.

The child health strategy working group met to review all the available information and survey findings. Priorities for service development were selected on the basis that they represented the best potential for purchasing health gain for the children of Tayside. Likewise, priority areas for resource release were identified on the basis that releasing resources from these areas would result in little or no loss of health benefit. A set of recommendations for service purchasing in Tayside was developed. 
The strategy was approved by the Health Board following public consultations and an area-wide 'child health forum', including representation from the main National Health Service child health professionals, trust providers and the Health Board. It was agreed that the strategy would be implemented. The observation was made that the recommendations won general support from local clinicians, even though high priority was given to investment in non-medical, low-technology services in the non-acute sector. It was felt that this reflected the strength of consensus that emerged from different data sources, particularly from the survey of professionals. It was felt that the strategic approach taken to priority setting, using the PBMA approach, resulted in a pattern of provision of health care giving a more rational and efficient allocation of scarce resources.

\subsubsection{Concluding comments on case studies}

These case studies are four very good examples of the use of different approaches to HNA. The Porirua City Health and Disability Report and Plan demonstrated a population-based approach, that involved consultation with the community, providers and local authority, but they also embarked on prioritisation. This is the type of HNA that DHBs were expected to undertake, and is a particularly good example of population-based HNA. The second case study of rapid appraisal in an urban setting, using a community-based approach, studied a deprived community in Bootle and used an intersectoral evaluation team to evaluate the community's health and social needs. The community undertook prioritisation of service requirements, and the priorities chosen were taken as a point of departure, with a view to formulating an action plan. Key to the success of this project were the strong linkages that were established between the community and planners, the latter having access to the resources necessary to address those needs. Rapid appraisal will not be used very often in the New Zealand context, yet it is a valuable tool to assess the health and social needs of a community, particularly when an intersectoral approach is required.

The third case study of an epidemiological-based HNA approach to renal disease is a very good example of the application of needs assessment to a disease entity, combining economic data regarding cost-effectiveness. In this sense it is not a 'pure' 
epidemiologically based case, but it does illustrate that approach. So far, there has not been any evidence of the use of this approach in New Zealand, yet there is scope to do this for diseases such as diabetes, coronary heart disease, and congestive heart failure, to name a few, given their local prominence. The fourth case study, the Tayside experience of PBMA, is a very good illustration of the application of health economics to a particular health service, in order to develop a strategy that clearly identified priorities for purchasing in child health. DHBs can use this example as a model for considering purchasing priorities regarding particular health services, and share the findings with other DHBs, in order to reduce duplication. The PBMA approach is explicit in defining a service, and it provides some answers for service reconfiguration, based on costeffectiveness. It is time consuming, and therefore cannot be undertaken on all services, but was reported to result in a pattern of health service provision that was described as more rational and allowed the efficient allocation of scare resources. It will be interesting to see whether, over the passage of time, DHBs undertake any of these types of HNA, beyond the mandated population-based HNAs. 
Chapter 4: Health Reforms: Policy Implications for Health Needs Assessment and Prioritisation

4.1 Introduction

4.2 Area Health Boards (1983-93)

4.2.1 Restructuring of hospital boards

4.2.2 Evolution of health needs assessment and prioritisation in AHBs

4.3 Quasi-market model (1993-97)

4.3.1 Reform from Area Health Boards to Regional Health Authorities

4.3.2 Evolution of health needs assessment and prioritisation in RHAs

4.4 Quasi-market model (1997-2000)

4.4.1 Reforms and the Health Funding Authority

4.4.2 Implications for health needs assessment and prioritisation

4.5 2000/01 Reforms (District Health Boards)

4.5.1 District Health Boards

4.5.2 New Zealand Public Health and Disability Act 2000

4.5.3 New Zealand Health Strategies

4.5.4 Health needs assessment policy environment

4.5.5 Implications for health needs assessment and prioritisation

4.6 Summary 


\subsection{Introduction}

New Zealand has experienced four sets of health reforms since the early 1980s. Successive governments have sought to change the way services are delivered in order to improve cost-effectiveness. Internationally, three broad approaches have been taken to health care reform; quasi-market (purchaser-provider split); managed competition; and managed care (Scott, 2001). New Zealand has implemented quasi-markets within a publicly funded health system, and has not managed competition. Managed care has been partially implemented as part of a social insurance scheme for managing accidentrelated care. Various approaches include population-based, community-based epidemiologically-based, corporate, comparative, and economic approaches, and efforts to establish a set of core of services accessible for all.

This chapter will consider whether the conditions established during each period of reform were consistent with effective health needs assessment. It discusses whether the current reforms are likely to result in more effective needs assessment and prioritisation than previously, and what factors are likely to affect the ability of the system to deliver on those goals.

\subsection{Area Health Boards (1983-93)}

\subsubsection{Restructuring of hospital boards}

Until the early 1980s, hospital care was organised through locally elected territorial hospital boards, of which there were 29. Public health services were delivered through district offices of the Department of Health. ${ }^{16}$ Primary care services were partially subsidised from the 1940s, but significant fee-for-service charges remained. The hospital board model was criticised for fragmented service planning and delivery, leading to significant differences between boards in the delivery of and access to services.

\footnotetext{
${ }^{16}$ The Department of Health was the predecessor to the present Ministry of Health.
} 
In 1983, the fourth Labour government amalgamated district public health services with hospital boards to create 14 territorial Area Health Boards (AHBs). These AHBs had a mixture of appointed and elected members, the majority being elected. They had responsibility for publicly funded hospitals and public health services, while primary care remained partially subsidised through the Department of Health. Boards were expected to achieve nationally-set targets for accountability for health care service delivery, in order to address some of the previous differences between hospital boards. Even so, equity of access could not be achieved because of lack of responsiveness to service users and to inefficiencies in the hospitals among other reasons. This was compounded by a lack of competition between hospitals (Gibbs, Fraser and Scott, 1988). Cost-effectiveness was limited by the separation of primary and secondary services and by inefficiency within the hospitals, which eventually led to the installation of managers in place of clinical leadership by medical superintendents and senior nurses.

\subsubsection{Evolution of health needs assessment and prioritisation in AHBs}

A review of the literature reveals that need for health care was assessed infrequently, and only by means of small projects within the AHBs, even though AHBs had responsibility for the health of the resident population. From all accounts, the old Area Health Boards did not directly engage in population-based health needs assessment. However they did conduct pre-assessment exercises, gathering epidemiological and health care service data related to their areas, for example; (Department of Health, 1993a, Department of Health, 1993b, Department of Health, 1993c, Department of Health, 1993d, Department of Health, 1993e, Department of Health, 1993f, Department of Health, 1993g, Department of Health, 1993h, Department of Health, 1993i, Department of Health, 1993j, Department of Health, 1993k, Department of Health, 19931, Department of Health, 1993m). 
This general lack of need assessment has been confirmed in discussions with a former AHB manager who held office in the Auckland area for an extended time. ${ }^{17}$ Services were generally provided on a historical basis, modified to some extent by the influence of locally elected board members, who were themselves influenced by lobby groups demanding more health care reflecting the particular needs of their members. There was no explicit process for prioritisation of purchasing, which again was likely to be influenced by lobby groups, including clinicians and members of the public.

\subsection{Quasi-market model (1993-97)}

\subsubsection{Reform from Area Health Boards to Regional Health Authorities}

The AHB model was criticised for lack of efficiency, mainly because there was no competition between AHBs, and their financial management was unsatisfactory. AHBs were poorly monitored and had a degree of operational freedom in that they were both purchasers and providers of services. For the first time there was explicit recognition of the dual nature of this. Fragmentation between primary and secondary care under the hospital board model continued under the AHBs.

In 1990 a new National Government proceeded to introduce sweeping health reforms based on the quasi-market model, with a purchaser-provider split and proposed competition between public and private purchasers and providers. The reforms made provision for the establishment of alternative health care plans, which would act as private purchasers in competition with four publicly funded Regional Health Authorities (RHAs) (Upton, 1991). In the event, alternative health care plans did not eventuate as the government chose not to allow competition to proceed. Instead a quasi-market developed, where the purchase of services was undertaken by public agencies on behalf of the public, rather than by patients themselves (J Le Grand and Bartlett, 1993). One example of public purchasing was community mental health services, which were

\footnotetext{
${ }^{17}$ Rod Perkins, former General Manager, Auckland Area Health Board, personal communication, 2002.
} 
purchased from non-governmental organisations, instead of being directly provided by the state.

Hospitals were organised into 23 Crown Health Enterprises (CHEs), designed to overcome certain weaknesses of the AHBs, including those of governance, accountability and performance. Elected boards were removed from the system, with central government now appointing all board members. In many cases, CHEs were monopoly providers in their regions. For the first time, purchasing of primary and secondary care was integrated, with the RHAs acting as purchasers of both.

A wider range of non-hospital providers, including Māori providers and primary care organisations, entered the system, taking advantage of the opportunity to contract for the supply of health services. Tendering for services also resulted in increased choice available to patients. For users of secondary care, however, this was offset to an extent by agreements between hospitals that admissions to various service departments would depend on the address of the patient and availability of the service that day at a particular hospital. Some competitive tendering led to savings in spending on hospital services (Lovatt, 1996). Equity of access improved considerably with the entrance of new service providers, mainly 'third sector' non-governmental organisations (NGOs). Māori providers focused predominantly on 'by Māori for Māori' services (Mays and Hand, 2000), improving the cultural acceptability of services and therefore access. It was also expected that the arrival of new providers would improve responsiveness and effectiveness. This happened for such services as health care for the elderly, maternity, mental health, and Māori health to name a few. New government funding was provided to foster the development of Māori providers.

Regional purchasers now had access to primary care funding and some chose to contract supply of primary care by capitation, rather than demand-driven fee-for-service arrangements. Innovation using a range of providers and greater flexibility in contracting allowed purchasers greater scope to offer service coverage in areas that previously had poor access to practitioners (Scott, 2001). More attention was paid to the 
quality of services delivered by providers, most of who were also required to be more accountable for service delivery than previously.

\subsubsection{Evolution of health needs assessment and prioritisation in RHAs}

The purchaser-provider split meant that RHAs were responsible for the purchase of health services for geographically defined populations. Some RHAs conducted population-based needs assessments for their defined populations, driven partly by the need to know more about their population (Northern RHA), and partly by enthusiasts who brought significant skills to the task, and provided an exemplar for the rest of the country (Midland RHA). The Midland RHA Health and Disability Analysis Unit was established for population-based assessment of health need and produced a series of high-quality reports included in the list in Appendix 3, p.298. Demographers, epidemiologists and statisticians staffed this unit and on the demise of RHAs the considerable body of experience within this unit was lost to the sector.

RHAs conducted various health needs assessments to assist with purchasing. The number of HNA publications by the RHAs indicates a considerable step-up from previous levels of HNA activity. As will be seen later, the number of HNAs that met the inclusion criteria for this research and were published during the four years 1997-2000 (25), matches the number published in the previous six years. Staff conducted other HNAs internally, wanting to obtain some idea of the need for a service, but not wanting to conduct a full-scale HNA. RHAs were also evaluating services and their costeffectiveness to a certain degree. At the same time as the RHAs were assessing health need, the Ministry of Health was also conducting national health needs assessments by collecting and analysing epidemiological data, mainly relating to morbidity and mortality.

The reforms emphasised population-based approaches to the prevention of disease and health promotion. In 1993, the government established the Public Health Commission, a Crown entity with a brief to monitor the state of the public health, to advise the Minister on public health, and to purchase or arrange for the purchase of public health (i.e. 
population-based) services. However it was disbanded in July 1995, probably because of perceived duplication of the role of the public health section of the Ministry of Health.

An important goal of the health reforms was to specify the access entitlement to publicly funded services. The government established the National Advisory Committee on Core Health and Disability Support Services, otherwise known as the Core Services Committee, ${ }^{18}$ with the task of establishing a list of core services that New Zealanders could rightfully expect to be publicly funded. The Committee was advisory, and there was no guarantee that the recommended list would be publicly funded.

The Committee defined core services as 'health and disability support services the Government should ensure are purchased, with due respect to its limited fiscal means, in order that people have access to effective services on fair terms.' (National Advisory Committee on Core Health and Disability Support Services, 1992, p.5) Emphasis was placed on equal access to core services rather than on equal outcomes or expenditure. The strategy was designed to ensure that those with limited resources could be assured of access to a defined range of services, and that others would be appropriately informed regarding the benefits of supplementary private insurance.

The Committee rejected a number of possible approaches to defining a core, including a positive list; a list of those services that would not be publicly funded; and a positive list with prioritisation as adopted in the state of Oregon (Kitzhaber, 1993). Instead, the government accepted a proposal by the Core Services Committee that the criteria under which services should be publicly funded for an individual were: '(1) benefit or effectiveness of the service; (2) value for money or cost-effectiveness; (3) fairness in access to and use of the resource; (4) consistency with the community's values.' (National Advisory Committee on Core Health and Disability Support Services, 1994, p.10). New and existing services for public funding were therefore to be judged on the

\footnotetext{
${ }^{18}$ The Core Services Committee subsequently became known as the National Health Committee in 1996.
} 
above criteria, which had been agreed following public consultation. These criteria were intended to be used by RHAs for prioritising purchasing (Minister of Health, 1995).

The Ministry of Health also established the New Zealand Guidelines Group that fostered the development of best-practice clinical guidelines for access to clinical care, based on clinical evidence. Prioritisation was implicit in these guidelines, but without specifically taking account of the availability of health resources.

Formal agreements with Māori iwi ${ }^{19}$ groups, acknowledging Treaty of Waitangi relationships, led to additional resources being provided for Māori service development. Previously the voice of Māori regarding their poor health status and need for more services appropriate for Māori had not been heard by non-Māori, who also controlled the public purse. Māori were invited to become co-purchasers with RHAs and did so in several instances (for example, in the Northern RHA). Māori health need was better recognised in response to the collection of epidemiological data demonstrating poor Māori health status, and high Māori health need. More funding and services were directed towards Māori health services, with encouragement being given to a range of Māori health providers. Community and Iwi provider groups proliferated as a consequence of increased Māori health service purchasing by RHAs. In addition, mainstream providers were required to provide better services for Māori people. ${ }^{20}$

\subsection{Quasi-market model (1997-2000)}

\subsubsection{Reforms and the Health Funding Authority}

Following the election of the National-New Zealand First coalition government in 1996, new reforms led to the coalescence of RHAs in 1997 into the Health Funding Authority

\footnotetext{
${ }^{19}$ Iwi is the name given to Māori tribal groups that are based in geographical districts throughout New Zealand.

20 'Mainstream providers' refers to the large group of traditional health service providers, the bulk of whom were non-Māori.
} 
(HFA) as a single purchaser of health services, mainly to achieve greater national consistency in access to services. The rhetoric of competition was replaced by an emphasis on co-operation. Crown Health Enterprises were renamed Hospital and Health Services (HHSs) to reflect the wider role of hospitals in health service provision in the community. The purchaser-provider split continued, with emphasis on the contracting process to shape service provision according to need, as determined by the purchaser following consultation.

During this period an even wider range of providers became available, including more Māori providers, increasing the choice available to the HFA as purchaser. Wide use of the tendering process allowed more providers to bid for service provision. More information on the services provided and the resources used to provide them allowed better informed purchasing decisions (Ashton, 1996). The HFA used its position as a monopoly purchaser of publicly-funded non-accident services (other than those contracted by the Ministry of Health directly) to shape contracts in order to make services more acceptable to users, especially Māori and low socio-economic groups. In at least one case, the tendering process led to private hospitals providing surgical services for hip and knee replacements in significant volumes, leading to an outcry from the public provider. ${ }^{21}$ Choice of efficient providers for additional contracts was hindered by concerns regarding geographical equity of access (Cumming and Salmond, 1998, p.122-146).

The quasi-market approach had its problems. The expectations that it would encourage flexibility, innovation and diversity were only partly realised, whilst waiting lists grew and disadvantaged populations found that their access to care, particularly primary health care, worsened. In an editorial by the Dean of the Christchurch School of Medicine, attention was drawn to a fundamental lesson of the health reforms - that a market approach to the delivery of health care has major limitations. 'The de-emphasis

\footnotetext{
${ }^{21}$ In 1997 Ascot Hospital, a private surgical facility in Auckland, was awarded a contract for hip and knee replacements from the Regional Health Authority. The public provider, Auckland Hospital and Health Services, complained bitterly that it was not offered the opportunity to contest the contract and felt that it should have been consulted first. The contract remained.
} 
during the reforms on public health and preventative measures must be reversed, with urgent strengthening of population-based strategies targeted toward preventable illness and injury, and toward those most in need.' (Hornblow and Barnett, 2000, p.134)

\subsubsection{Implications for health needs assessment and prioritisation}

Assessment of health need became more common during this period, because internal HFA policies often required an assessment of health need prior to contracting new services. Almost certainly this arose because of the differences in performance between the four RHAs, and the new approach resulted in standardisation. In addition, the HFA was a somewhat technocratic organisation, and endeavoured to take the politics out of health. The transition to more explicit processes signalled a move towards an evidencebased approach to funding, but many services were still not subject to needs assessment. Those that were conducted and published by the HFA, Public Health Commission and the Ministry of Health are considered elsewhere in more detail (see Chapter 6, p.149).

Waiting times for elective services came on to the agenda because of prolonged delays and significant variations in access to services throughout the country. Hoping to achieve equity of access, the Ministry of Health developed a surgical booking system utilising priority criteria. It was considered that clarification and uniformity of public entitlement would lead to fairer prioritisation. More formal assessment systems promote equity by ensuring that those most in need and able to benefit from surgical services are given priority in access to service (Scott, 2001). This was a form of personal health needs assessment with rationing of services using nationally agreed access criteria. It was similar to the process used for those seeking access to disability support services.

The HFA developed a prioritisation framework for allocating new investment in health care services. New principles for prioritisation adopted by the HFA included effectiveness, cost, equity, Māori health, and acceptability (see Section 2.4.4, p.42). The HFA recognised that addressing health inequalities required investment in targeted services and populations to achieve equity. Prioritisation therefore took account of equity as well as cost-effectiveness, and was particularly applied to developing a group of projects for investment as new funding became available, in which marginal health 
need was assessed and prioritised. Funding agreements targeted those with low socioeconomic status as well as Māori, child, mental health and physical environmental services. Considerable attention was given to devising robust systems for priority setting, and to determining cost-effectiveness, including some work on the use of $\mathrm{QALYs}^{22}$ to measure effectiveness at the margin across health services.

The HFA also consulted more widely with the community than it predecessors, and set in place formal processes for community consultation (Health Funding Authority, 2000b). Greater community involvement with HNA was generally sought with one important example being that of the Porirua Kapiti Healthlinks Project which revealed high community need. In response to the findings, the government decided to establish a Health Action Zone in the Porirua-Kapiti district and provide additional resource to improve access to certain services (Porirua Kapiti Healthlinks Project, 2000b).

Engagement with Māori was fostered by the HFA which developed Memoranda of Understanding with Treaty partners, based on the Treaty of Waitangi principles regarding partnership, participation and protection. Māori were regularly consulted regarding the provision of services to Māori. The HFA formed a Māori Health Operating Group, which was responsible for actively promoting Māori health workforce development, and for fostering of service contracts with ‘for Māori by Māori' providers.

\subsection{0/01 Reforms (District Health Boards)}

\subsubsection{District Health Boards}

The Labour-Alliance coalition government elected in 1999 introduced a set of health reforms that included the formation of 21 DHBs, with boundaries geographically aligned with those of groups of district councils (see Appendices 1 and 2, p.296). A mixture of seven democratically elected and four Ministerially appointed members

\footnotetext{
${ }^{22}$ QALY (Quality-adjusted life year) is a year of life adjusted for the health-related quality of that year.
} For further information refer to the Glossary at the front of the thesis. 
govern boards, each of which must include two Māori members, within the total of 11. Each Board has three statutory committees (Hospital Advisory Committee, Community and Public Health Advisory Committee, and the Disability Support Services Advisory Committee), as well as various other committees.

There are significant opportunities for Māori involvement, at several levels. Specific representation at board level for the first time gives Māori a governance role, recognising Treaty of Waitangi principles. DHBs are required to consult with local Māori when planning services, including consulting on the District Strategic Plan. DHBs are also proactive in seeking Māori health providers to provide services for Māori people.

DHBs previously funded on a historical basis will shortly be funded on a population basis, weighted according to need. From 2004/05 the formula reflects age, gender, ethnicity, and deprivation; and boards will also receive funds reflecting unmet need, rurality and diseconomies of scale. ${ }^{23}$ The new funding will be introduced over time, taking account of historical funding, so that Boards have time to adjust smoothly to new funding lines. Some boards will receive more funding than in the past, but the funding to others will not increase at the same rate as previously, until equity between DHBs is reached according to the formula.

DHBs are to be responsible for providing hospital services through their provider arms, and for funding non-hospital services. Thus, the funder-provider split has been retained for most services, except those provided through the hospital. Each DHB in the future is to be responsible for purchasing all services for its population, including personal health services, Māori health services, mental health services, disability support services, and public health services. Boards became responsible for these services on 1 January 2001, except for disability support services on 1 October 2003 and public health services that are proposed to be devolved partly to DHBs at a later date. Mental health secondary

23 'Diseconomies of scale' refers to economic disadvantage caused by small size or remoteness, or by provision of tertiary services. 
care services are purchased regionally, with DHBs co-operating for their provision and purchase. The Ministry is currently exploring means of purchasing those mental health services that fall outside of the Mental Health Blueprint (Mental Health Commission, 1998). ${ }^{24}$

The Government shaped its direction for the health of New Zealanders with the publication of the New Zealand Health Strategy and the New Zealand Disability Strategy which focus on improving the health and independence of New Zealanders (Minister of Health, 2000d, Minister of Health, 2001b). In addition to long-term health goals, the New Zealand Health Strategy sets out a number of key objectives for immediate action (e.g. reducing smoking, reducing the incidence and impact of cancer, reducing disparities in health between Māori, Pacific peoples and other New Zealanders), and key service priority areas for particular attention (e.g. public health, primary health care, reducing waiting times for public hospital elective services). These are discussed in more detail later. In addition to these two strategy documents, the government has also published other key strategies including the Primary Health Care Strategy, the Māori Health Strategy, and the Pacific Health Strategy (Minister of Health, 2001b, Minister of Health, 2001c, Minister of Health, 2001a, Minister of Health, 2002).

Many DHBs commenced with operating deficits, which generally increased during the 01/02 year. However, Government increased funding for DHBs for the 02/03 year, with total base funding of $\$ 5.515$ billion that year, and notified increases of $\$ 337$ million and \$208 million for the 03/04 and 04/05 outyears respectively. Government has made it clear to DHBs that it will not agree to District Annual Plans from DHBs that do not plan a zero deficit for the $03 / 04$ year. This means that while DHBs are receiving additional funding to address the issue of ongoing operational deficits, and at the same time are moving towards full population-based funding, they need to operate more efficiently to meet the zero deficit target. DHBs are required to meet this target without any service reductions, unless approved by the Minister of Health. A number of DHBs are finding

24 The 'Blueprint' covers those services provided by hospitals and health services for the seriously mentally-ill (3\% of the population), but not the balance of mental health services provided in the community (to another $15 \%$ of the population). 
this to be a challenging requirement. Others have already met the target quite successfully, owing to better starting positions.

\subsubsection{New Zealand Public Health and Disability Act 2000}

Clause 3 of the New Zealand Public Health and Disability Act 2000 states that the Act has, in part, the following purpose:

\section{Purpose}

(1) The purpose of this Act is to provide for the public funding and provision of personal health services, public health services, and disability support services, and to establish new publicly-owned health and disability organisations, in order to pursue the following objectives:

(a) to achieve for New Zealanders -

(i) the improvement, promotion, and protection of their health

(ii) the promotion of the inclusion and participation in society and independence of people with disabilities

(iii) the best care or support for those in need of services

(b) ...(Minister of Health, 2000e, p.4)

Clause 3(2) of the Act is explicit in stating that the aims of the Act should be achieved within the amount of funding provided and it therefore implies prioritisation:

(2) The objectives stated in subsection (1) are to be pursued to the extent that they are reasonably achievable within the funding provided. (Minister of Health, 2000e, p.5)

The requirements for health needs assessment are explicitly stated in clause 23(1)g:

\section{Functions of DHBs}

(1) For the purpose of pursuing its objectives, each DHB has the following functions:

(g) to regularly investigate, assess, and monitor the health status of its resident population, any factors that the DHB believes may adversely affect the health status of the population, and the needs of that population for services 
(k) to provide information to the Minister for the purposes of policy development, planning, and monitoring in relation to the performance of the DHB and to the health and disability support needs of New Zealanders

...(Minister of Health, 2000e, p.24)

Clause 23(k) above requires that DHBs provide information to the Minister regarding the health and disability support needs of New Zealanders.

Clause 38(3)(a) repeats the requirement for health needs assessment, in the context of strategic planning:

\section{District strategic plans}

(3) Before a DHB determines or makes a significant amendment to a district strategic plan, it must -

(a) assess the health status of that population, any factors that the DHB believes may adversely affect the health status of that population, the needs of that population for services, and the contributions that those services are intended to make towards the health outcomes and health status sought for that population; and ... (Minister of Health, 2000e, p.32)

Subsection 38(3)(b) requires DHBs to consult on district strategic plans.

(3) Before a DHB determines or makes a significant amendment to a district strategic plan, it must -

(b) prepare a draft plan or amendment and consult its resident population on that draft. (Minister of Health, 2000e, p.33)

Schedule 4(2) of the Act also provides that DHBs will receive advice from Community and Public Health improvement Advisory Committees (CPHAC) regarding the health needs of the resident population:

2 Functions of community and public health improvement advisory committees 
(1) The functions of the community and public health improvement advisory committee of the board of a DHB are to give the board advice on -

(a) the needs and any factors that the committee believes may adversely affect the health status of the resident population of the DHB; and

(b) priorities for use of the health funding provided.

(2) The aim of a community and public health improvement advisory committee's advice must be to ensure that the following maximise the overall health gain for the population the committee serves:

(a) all service interventions the DHB has provided or funded or could provide or fund for the care of that population:

(b) all policies the DHB has adopted or could adopt for the care of that population.

(3) A community and public health improvement advisory committee's advice may not be inconsistent with the New Zealand Health Strategy (Minister of Health, 2000e, Schedule 4(2), p.112).

In summary, the new Act is quite specific and requires DHBs, with respect to their resident populations, to:

- conduct health needs assessments

- $\quad$ prioritise within the funding provided

- consult on the strategic plan

\subsubsection{New Zealand Health Strategies}

There are two main health strategy documents that guide the health sector regarding the way that health services will be delivered under this government. They are the New Zealand Health Strategy and the New Zealand Disability Strategy. Whilst there are a number of other health strategies (up to 38 at the last count), these two are the founding documents. The New Zealand Health Strategy includes a set of principles to guide the future development of the health sector:

- Acknowledging the special relationship between Māori and the Crown under the Treaty of Waitangi

- Good health and wellbeing for all New Zealanders throughout their lives

- An improvement in heath status of those currently disadvantaged 
- Collaborative health promotion and disease and injury prevention by all sectors

- Timely and equitable access for all New Zealanders to a comprehensive range of health and disability services, regardless of ability to pay

- $\quad$ A high-performing system in which people have confidence

- Active involvement of consumers and communities at all levels (Minister of Health, 2000d, p.vii)

The Strategy also includes goals and objectives towards improving the health of the community, and reducing differences in health status between population groups. The Government has selected 13 key priority population objectives for the Ministry of Health and DHBs to focus on for immediate action:

- reducing smoking

- improving nutrition

- reducing obesity

- $\quad$ increasing the level of physical activity

- $\quad$ reducing the rate of suicides and suicide attempts

- minimising harm caused by alcohol, and illicit and other drug use to individuals and the community

- $\quad$ reducing the incidence and impact of cancer

- reducing the incidence and impact of cardiovascular disease

- reducing the incidence and impact of diabetes

- improving oral health

- reducing violence in interpersonal relationships, families, schools and communities

- $\quad$ improving the health status of people with severe mental illness

- $\quad$ ensuring access to appropriate child health care services including well child and family health care and immunisation. (Minister of Health, 2000d, p.13)

The Government seeks to reconfigure the health and disability sector to improve the overall health of New Zealanders. Government also proposes improving access to public health services, primary health care services, public hospital services and mental health services in the short to medium term. It states that 'The introduction of District Health Boards will help to ensure that the services reflect the needs of individuals and communities at a local level' (Minister of Health, 2000d, p.3). District Health Boards will be responsible for the health of their populations. 
The New Zealand Health Strategy states that:

The changes will:

- focus on population needs

- $\quad$ reduce disparities in health

- $\quad$ emphasise community and consumer involvement at all levels

- improve co-ordination across the health sector so that the whole system works for people ... (Minister of Health, 2000d, p.3)

The implications for DHBs needs assessments are that they will need to take account of priority goals, objectives, targets and performance indicators contained within the New Zealand Health Strategy and the New Zealand Disability Strategy. Specifically, Crown Funding Agreements with DHBs are expected to include performance measures for DHBs regarding key priority objectives.

\subsubsection{Health needs assessment policy environment}

In addition to the legislation referred to above, the Ministry of Health sets the policy environment for HNAs operationally. The Ministry published a paper entitled 'Health Needs Assessment for New Zealand: An overview and guide,' in 2000, which set out the general requirements. This Ministry paper notes that HNAs provided opportunity for:

- $\quad$ describing the health care needs of the population covered by the DHB, being geographically defined by several local authority boundaries, and the differences between district, regional and national populations

- $\quad$ obtaining data from primary health care regarding enrolled ('affiliated') populations

- $\quad$ learning about the broad health needs and priorities of communities through community consultation

- $\quad$ highlighting the areas of unmet needs, ascertaining whether there are health sector responses that are effective, and prioritising these within the health needs assessment exercise

- deciding rationally how to prioritise the use of resources to maximise health gain, and distribution of health gain, to improve the health of the included population in the most effective and efficient way

- $\quad$ influencing policy, inter-agency collaboration and co-ordination, as well as promoting evaluation, research and development priorities. (Ministry of Health, 2000b, p.5) 
The Ministry then outlined their view of the planning cycle for DHBs diagrammatically in order to demonstrate the role of health needs assessment. This is shown in Figure 6 below, which illustrates a comprehensive planning model with tasks to be completed in sequence shown.

Figure 6: DHB Planning Cycle

\section{Parts covered by this thesis}

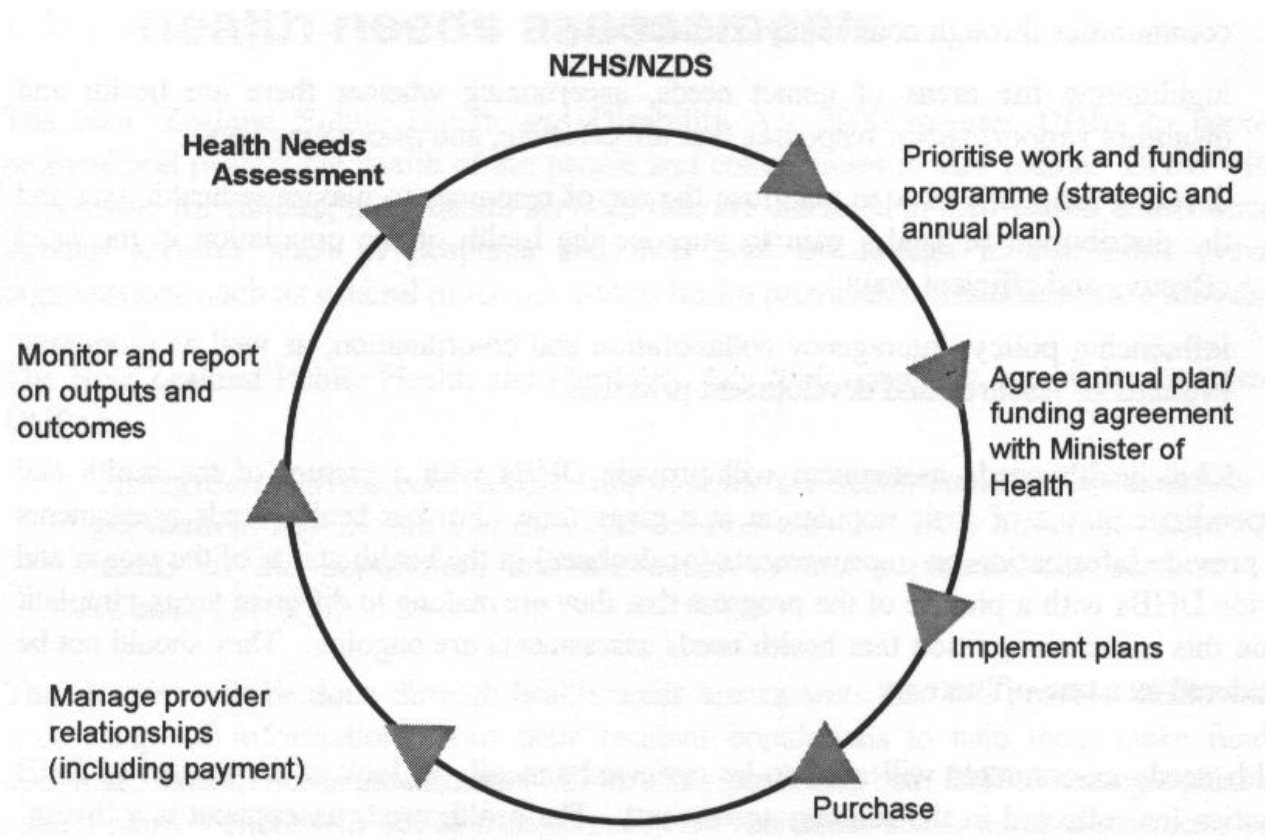

Source: (Ministry of Health, 2000b, p.6)

The Ministry also agreed with the schematic representation in Figure 7 initially shown in the background paper and literature review sent out to DHBs to assist them with the planning process (Coster, 2000). 


\section{Figure 7: Planning pathway}

\section{Government's New Zealand Health Strategy and New Zealand Disability Strategy}

These strategies will provide the overarching policy framework within which the health and disability sector will be expected to operate. These strategies will establish specific nation-wide health and disability outcome goals, objectives and targets, and sector performance standards.

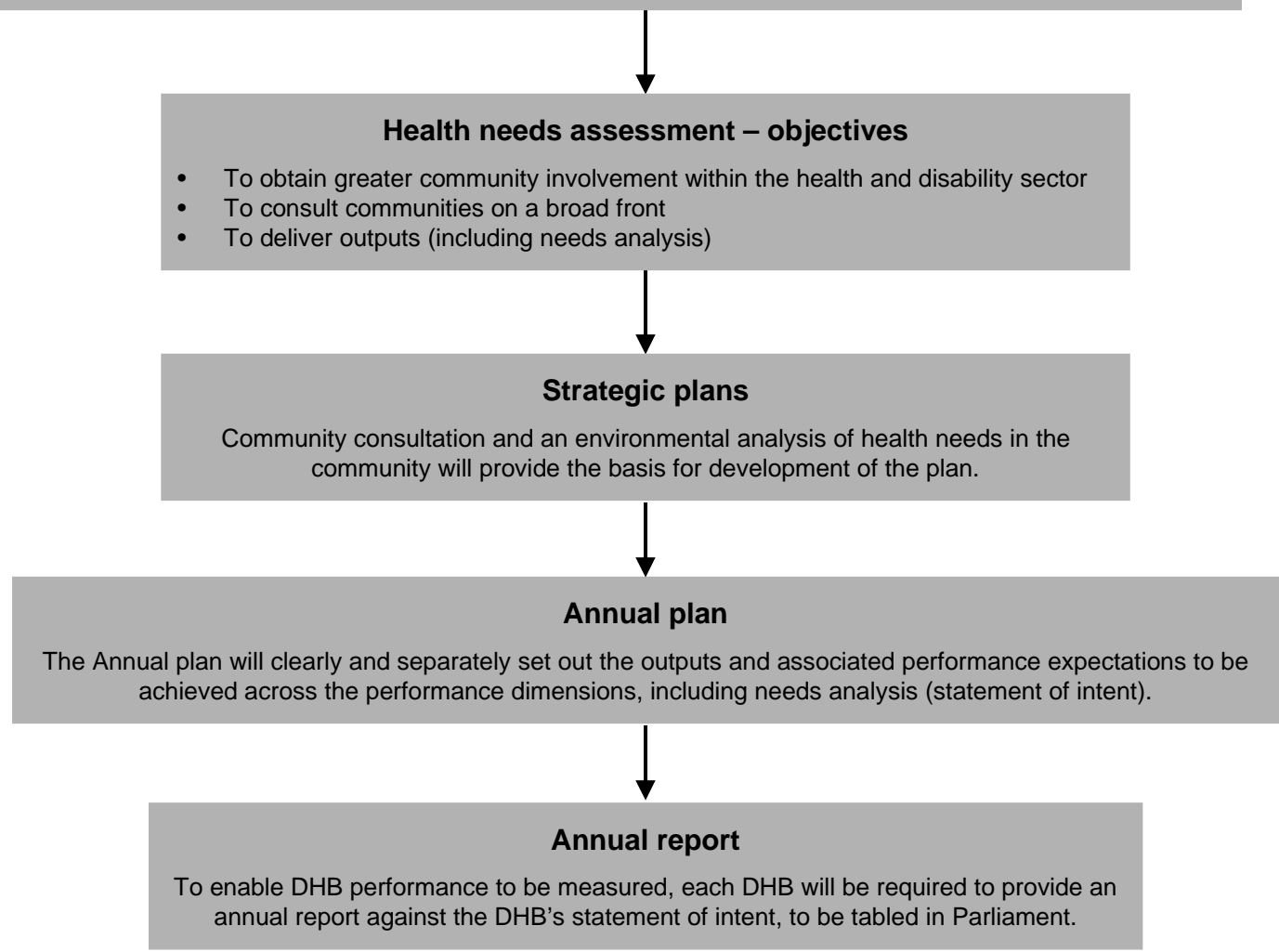

Using these figures as a basis, but also drawing on the Ministry advice referred to above, the sequence of events in the planning and purchasing process is made up of the following steps:

1. DHB defines health needs assessment programme objectives.

2. DHB conducts health needs assessments in district, with involvement of Māori; consults with the public for prioritisation (function of CPHAC).

3. DHB reviews the health needs assessment (including prioritisation) and agrees to fund/purchase to a certain level.

4. DHB prepares strategic plan and annual plan and consults with community. 
5. Strategic and annual plans are approved by the Minister of Health.

6. Annual plan is resourced through Vote: Health and the Funding Agreement with the Crown.

7. DHB implements health services according to the annual plan.

8. Evaluation of effectiveness of health services delivery against annual plan and performance measures contained within Funding Agreement.

Finally, in addition to the published policies, the Ministry also provides DHBs with an Operating Policy Framework (OPF), which establishes further Ministry requirements for the operation of DHBs. The OPF specifies various procedures that DHBs are to follow for operational matters. DHBs are therefore operating in an environment that is moderately well controlled by the Ministry of Health. This is not necessarily bad for DHBs and ensures consistency across all DHBs. In particular, such a controlled environment would be expected to lead to some consistency regarding the impact of HNA and prioritisation on planning and purchasing. On the other hand, there is sufficient flexibility for there to be significant differences between DHBs, as shall be seen later.

\subsubsection{Implications for health needs assessment and prioritisation}

Assessment of health care need was mandated in the New Zealand Public Health and Disability Act 2000. DHBs are required to conduct health care needs assessments of their populations. All completed these assessments by November 2001, in time for incoming elected boards. Health need assessments were important to allow the DHBs to understand the health needs of their populations, and prepare District Strategic Plans (DSPs) and District Annual Plans (DAPs) based on the resulting information. The process allowed input from communities into the health needs assessment, using community data, surveys, focus groups and key informant interviews. All this information, along with epidemiological data collected by DHBs, formed the raw material of the health needs assessments. In some cases DHBs identified social care need, and have raised issues with local councils to enlist their support in addressing these needs. 
Variation between DHBs in their assessment and prioritisation of health needs is to be expected, just as there were variations among RHAs and AHBs. Just how significant those differences are will become apparent during the course of this research.

DHBs were also required by the Ministry of Health to develop prioritisation frameworks and submit them for approval before purchasing (Ministry of Health, 2000b). The Ministry assisted with the process by supplying DHBs with the model developed by the HFA, and its adaptation by the Ministry (Health Funding Authority, 2000d, Health Funding Authority, 2000e). DHBs were expected to apply their prioritisation framework to new spending, and if possible, to prioritise existing spending. Given that the new funding largely amounted to that for demographic growth and tagged primary care and mental health, DHBs found limited use for the prioritisation framework in the first instance. Most boards were reluctant to reprioritise - that is, to disinvest in existing services, - although some made changes at the margin. Another challenge for DHBs was acknowledging national health priorities, as set out in the New Zealand Health Strategy, while trying to achieve local responsiveness (Minister of Health, 2000d). Details of the research findings in this area follow later. Consumer involvement is expected to increase through such democratic channels as locally elected DHBs, community involvement in health needs assessments, and prioritisation, and input into DSPs through public consultation. Research regarding community consultation is outside the scope of the present research but forms part of the larger Health Reforms 2001 research project.

\subsection{Summary}

The effects of the health reforms on HNA and prioritisation including Māori and consumer involvement from 1983-2003 are summarised in Table 8 (p.117). There was an evolution of HNAs from minimal, if any, activity by AHBs through to purposive activity by some RHAs, notably Midland RHA who set up a Health and Disability Analysis Unit and conducted a whole programme of HNAs. The HFA conducted a modest number of HNAs, in order to base purchasing on assessed need, and to clarify need for services where there was conflicting evidence. The prioritisation process 
developed by the HFA was the first explicit application of a prioritisation framework to service purchasing by a purchaser of population-based health services in New Zealand. DHBs were explicitly mandated to conduct population-based assessments for their geographically defined populations. In future, it is expected that prioritisation will follow a new explicit process, which is currently under development by DHBs and the Ministry of Health. ${ }^{25}$

DHBs operate as independent Crown Entities, but are fairly well-controlled by government and the Ministry of Health. This control operates at various levels, including legislation, policy directions, Operating Policy Framework, Service Coverage Agreements, Performance Monitoring Frameworks, approval of District Strategic and Annual Plans, and Crown Funding Agreements. The argument is not that such a controlled environment is unhealthy, but how that environment allows DHBs to be responsive to local needs. In fact, there is good support for the proposition that controls are desirable in order to maintain a degree of national consistency, but also that DHBs need to be responsive to local needs. That responsiveness and the degree of national consistency achieved in planning and purchasing processes will be explored later.

${ }^{25}$ As at December 2003. 
Table 8: Health reforms and effects on health needs assessment, prioritisation, including Māori and consumer involvement $1983-2003$.

\begin{tabular}{|c|c|c|c|c|}
\hline & HNA & Prioritisation & Māori involvement & Consumer involvement \\
\hline $\begin{array}{l}\text { Area Health Boards } \\
(1983-93)\end{array}$ & $\begin{array}{l}\text { Anecdotally, use with only a few } \\
\text { projects. }\end{array}$ & $\begin{array}{l}\text { No explicit use of prioritisation } \\
\text { process. }\end{array}$ & $\begin{array}{l}\text { No specific acknowledgement } \\
\text { of role in health services. }\end{array}$ & $\begin{array}{l}\text { Elected health boards allowed } \\
\text { public input. }\end{array}$ \\
\hline $\begin{array}{l}\text { Quasi-market (1993-97) } \\
\text { (RHAs) }\end{array}$ & $\begin{array}{l}\text { Mainly projects to determine } \\
\text { need for specific services; } \\
\text { exceptions were two RHAs both } \\
\text { of which conducted a } \\
\text { programme of population-based } \\
\text { needs assessments for their } \\
\text { population. }\end{array}$ & $\begin{array}{l}\text { Some RHAs prioritised according } \\
\text { to need; no explicit purchaser } \\
\text { processes or framework. Core } \\
\text { Services Committee endeavoured } \\
\text { to determine core of publicly } \\
\text { funded services; advised on } \\
\text { principles for prioritisation of } \\
\text { services; promoted guidelines; and } \\
\text { developed surgical waiting list } \\
\text { criteria-based booking system. }\end{array}$ & $\begin{array}{l}\text { RHAs formed Treaty } \\
\text { relationships with Māori, in } \\
\text { some instances becoming co- } \\
\text { purchasers; Māori Provider } \\
\text { Organisations (MAPOs) } \\
\text { fostered; Māori participation } \\
\text { more often sought. }\end{array}$ & $\begin{array}{l}\text { Board members all appointed; } \\
\text { Some public consultation on } \\
\text { strategic plans, and on } \\
\text { guidelines for health care. }\end{array}$ \\
\hline $\begin{array}{l}\text { Quasi-market (1997-2000) } \\
\text { (HFA) }\end{array}$ & $\begin{array}{l}\text { Increasingly wide range of } \\
\text { policies and services subject to } \\
\text { needs assessment. }\end{array}$ & $\begin{array}{l}\text { Prioritisation framework developed } \\
\text { using National Health Committee } \\
\text { principles; framework generally } \\
\text { applied to new projects seeking } \\
\text { funding ('top-drawer' projects). }\end{array}$ & $\begin{array}{l}\text { Treaty relationships fostered, } \\
\text { more MOUs with Māori iwi; } \\
\text { Māori provider development } \\
\text { promoted by Māori Health } \\
\text { Operating Group; Wide range of } \\
\text { Māori health providers } \\
\text { contracted for services. }\end{array}$ & $\begin{array}{l}\text { Public consulted on strategic } \\
\text { plans, and service requirements; } \\
\text { Formal consultation processes } \\
\text { developed to foster proper } \\
\text { consultation. }\end{array}$ \\
\hline 2000/01 Reforms (DHBs) & $\begin{array}{l}\text { Population-based health care } \\
\text { need assessment mandated by } \\
\text { legislation; theoretically linked } \\
\text { to prioritisation, District } \\
\text { Strategic Plans and Annual Plans } \\
\text { and service purchasing; } \\
\text { Communities to be consulted as } \\
\text { part of the process. }\end{array}$ & $\begin{array}{l}\text { DHBs each required to develop } \\
\text { their own explicit prioritisation } \\
\text { framework, based on Ministry } \\
\text { advice; Ministry also set directions } \\
\text { in the New Zealand Health } \\
\text { Strategy, with national priorities; } \\
\text { set challenges for DHBs } \\
\text { responsible for local } \\
\text { responsiveness in an environment } \\
\text { of significant central government } \\
\text { control. }\end{array}$ & $\begin{array}{l}\text { Māori representation on DHBs } \\
\text { mandated by statute; DHBs } \\
\text { required to consult with local } \\
\text { Māori; Mana Whenua } \\
\text { relationship with DHBs. }\end{array}$ & $\begin{array}{l}\text { Boards are mixture of elected } \\
\text { and appointed members; Board } \\
\text { meetings held in public; Public } \\
\text { required to be consulted on } \\
\text { DSPs; Public consulted on } \\
\text { health care needs during HNAs } \\
\text { by DHBs }\end{array}$ \\
\hline
\end{tabular}

Abbreviations: DHB, District Health Board; MOU, Memorandum of Understanding; MOH, Ministry of Health; PHC, Public Health Commission; NHC, National Health Committee (National Advisory Committee on Health and Disability Services); RHA, Regional Health Authority; HFA, Health Funding Authority. 


\section{Chapter 5: Methods}

\subsection{Introduction}

5.2 Qualitative methods applicable to the current research

\subsection{Research Design}

5.3.1 Part 1. Literature Review

5.3.2 Part 2. Evaluation of health needs assessments (1991-2000)

5.3.3 Part 3. Evaluation of health needs assessments and prioritisation undertaken by the DHBs

5.3.4 Part 4. Evaluation of the effectiveness of government policy regarding health needs assessment, prioritisation, and planning 


\subsection{Introduction}

Policy reforms such as the formation of DHBs invite evaluation, particularly of process, and subsequently impact assessment. However, research into complex reforms while those reforms are being implemented is difficult. Reasons for this include the difficulty of defining reform objectives (Le Grand, Mays and Mulligan, 1998, Organisation for Economic Co-operation and Development, 1994, Orsman, 1999, Robinson and Le Grand, 1994); the impossibility of researching full-coverage reforms using experimental or quasi-experimental methods (Rossi, Freeman and Lipsey, 1999); the difficulty of assessing the impact of such reforms on the achievement of health goals; difficulties in using performance data relating to efficiency goals when the data are of poor quality, incomplete or even unavailable (Drummond, 1995); and the fact that often 'no governmental system of monitoring and evaluation is set up alongside,' health reforms (Robinson and Le Grand, 1994, p.243). Evaluation is further complicated by other policy changes that occur in a health system during the evaluation period - changes which will undoubtedly have an impact on any indicator that might otherwise be used to monitor progress of the reforms (Drummond, 1995).

A quasi-experimental approach might be developed to evaluate the performance of individual DHBs (Rossi, Freeman and Lipsey, 1999). Such a method was not possible for this research however, as setting up such an evaluation would involve specifying at least two different approaches to HNA, prioritisation and planning, which would be tested by different DHBs, but the reform agenda requires all DHBs to take the same approach to these processes.

Before-and-after studies were the most suitable method, since the reforms were fullcoverage reforms (Rossi, Freeman and Lipsey, 1999). Ideally the evaluation would be conducted over an extended period to determine changes in health outcomes. However, it would be difficult to attribute change to particular reforms, given the confounding impact of current events. 
Researching the impact of the HNA policy on planning and purchasing was therefore challenging. The approach chosen was that of an evaluation framework (Ovretveit, 1998, Vedung, 1997). The evaluation methodology was largely qualitative, designed to explore the processes and outcomes of the health reforms as well as analysing the experiences of key stakeholders in the health sector. It also compared and contrasted the impact of HNAs with that of assessments conducted prior to the current health reforms.

A qualitative approach was an appropriate methodology for research that sought to understand the effect of the reforms on behaviours and relationships. Such qualitative stakeholder research has been widely used in the past, in New Zealand and overseas (Le Grand, Mays and Mulligan, 1998, Mays N. et al, 1998, Robinson and Le Grand, 1994). As this was a before-and-after study, it was important to acquire detailed knowledge of HNAs conducted under the previous model of the purchaser-provider split. The research also sought information regarding the attributes, successes and failures of past HNAs, which were conducted at a time when there was little policy in this area. The research also looked for potentially confounding policies, so as to assess more accurately the contribution the actual reforms make to HNAs and their impacts.

An evaluation framework considers the objectives, processes and outcomes of a policy or programme. Simply considering outcomes, whether intended or unintended, does not in itself evaluate whether a programme is working. Even if desired outcomes were achieved, it should be asked 'what features of the policy or programme allowed those outcomes to be achieved?' An evaluation framework allows consideration of how and to what extent different kinds of data converge on a single programme event. But it also defines each programme element in relation to its antecedents and consequences. A programme element is therefore interpreted not in isolation, but in the light of its expected relationships. This is particularly relevant to a DHB programme of planning and purchasing, where the question is 'how well are the elements of that programme connected or 'joined up'?' Specifically, how does the HNA influence prioritisation, is the prioritisation reflected in District Strategic Plans, and then in District Annual Plans and then in purchasing? What is the effectiveness or impact of HNA on the planning and purchasing process? The policy for the use of HNA and prioritisation was defined 
as a comprehensive planning model (Ministry of Health, 2000b, p.6). The connections between elements of the model are shown below. 
Figure 8: Comprehensive rational planning model applied to DHB health service purchasing.

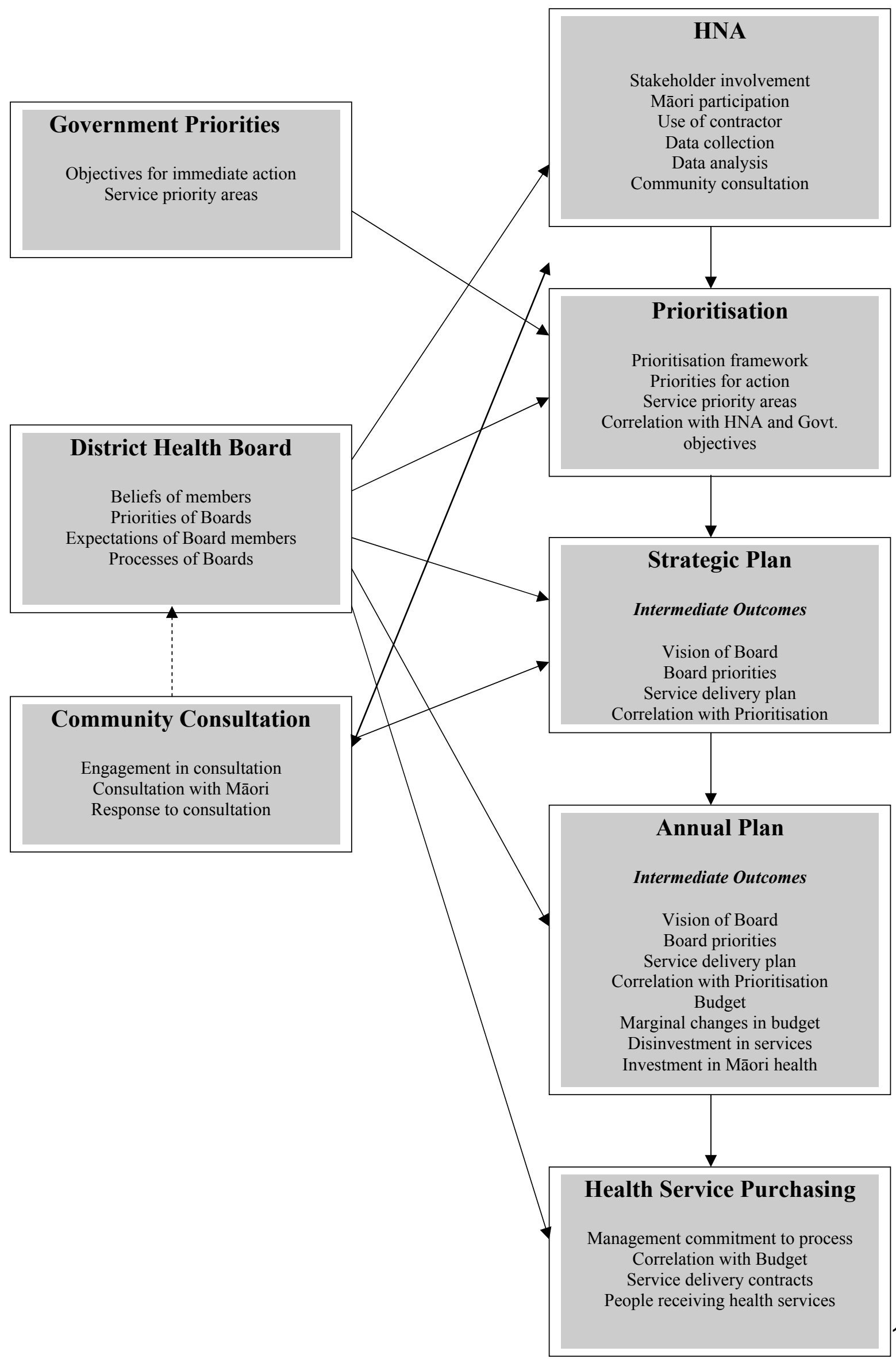


This model includes the collection of HNA data, key recommendations being prioritised by DHB boards, and then the incorporation of priorities into DSPs and DAPs.

In order to evaluate this model, various data can be collected regarding each component, or stage, of the process. Potential data sources available for evaluation are presented in Table 9 below, and those that were used for the research are marked with an asterisk.

Table 9: Comprehensive rational planning model for DHBs and data sources available for evaluation.

Components and questions
Government policy and expectations
What is the Government's policy and
expectations regarding HNA policy, including
prioritisation?
District Health Board
What are the expectations of Boards regarding
progress in next 2-3 years? Was CPHAC
involved?
HNA
Was a contractor involved? Was there
stakeholder involvement? Māori participation?
What range of data was collected? Any new
community data? Community consultation?

\section{Prioritisation}

What inter-board variation was there for prioritisation frameworks? What local variation from Govt priorities was there (objectives and service priority areas)?

How did the prioritisation correlate with the HNA?

\section{District Strategic and Annual Plans}

How do the Strategic and Annual Plans correlate with prioritisation (and HNA)? Is this reflected in the proposed Budget? Is there new investment in Māori health?

\section{Community consultation}

What level of community consultation has there been?

\section{Health service purchasing}

Has the assessment of need resulted in health service delivery contracts to meet those health needs?

\section{Potential main data sources for evaluation \\ NZ\&PHD Act 2000*; Cabinet Memorandum*; NZ Health Strategy*; Interview with Minister of Health; Interviews with Ministry of Health officials*; Hansard.}

Survey of expectations of Board Members, if available aggregated by board. Board and CPHAC minutes.

\section{HNAs *}

Interviews with DHB Funding and Planning managers*; Board Agendas and Minutes;

Interviews with DHB Chief Financial Officers; Interviews with CPHAC Chairs;

Surveys of Board Members.

Board Minutes. Also stated in Strategic Plan and/or Annual Plan* Govt priorities stated in NZ Health Strategy.* HNAs available publicly.* Minister's expectations of DHBs, and Service Coverage Schedules. * Operational Policy Framework. *

\section{District Strategic and Annual Plans.*}

Budget in Annual Plan.*

Annual reports, and survey and/or interviews with Funding and Planning managers*. Extent of consultation, evidence of consultation with Māori.* Evidence of incorporation of feedback from consultation*

Evidence of new contracts. (although existing contracts may also deliver the outputs); Analysis of budgets;

Survey and/or Interviews with Funding and Planning managers.*

NB: Those marked with an asterisk represent data sets actually collected in the course of this research.

The three main sources of data are (1) multiple document analysis, (2) interviews with 20 Planning and Funding Managers, and (3) two interviews with Ministry of Health officials. The methods used to collect the various data are now described in detail. 


\subsection{The application of qualitative methods to the current research}

Qualitative research methods have a long history in the social sciences and deserve to be an essential component in health and health services research. (Pope and Mays, 1995, p.42)

Pope and Mays (1995) assert the value of qualitative methods, in a climate where 'qualitative and quantitative approaches to research tend to be portrayed as antithetical' (p.43), a tendency they reject demonstrating that qualitative techniques can complement quantitative research, and, they suggest, extend its scope: 'The goal of qualitative research is the development of concepts which help us to understand social phenomena in natural (rather than experimental) settings, giving due emphasis to the meanings, experiences, and views of all the participants' (p. 43). Qualitative research offers insight into experiential phenomena, to help determine answers to such questions as what? how? and why? Qualitative techniques are appropriate for use by health services and policy researchers to collect information on the way that services and policies have been implemented. They can be used in their own right, to supplement quantitative data using triangulation methods (Bowling, 1997, Lincoln and Guba, 1985, Patton, 1999, Stake, 1995), or to complement quantitative work by exploring complex phenomena or areas not amenable to quantitative research.

The place of qualitative research methods in health services research, and particularly in the evaluation of such work, has been frequently reviewed (Beech, Guilliford, Mays, Melia and Roderick, 1997, Dingwall, Murphy, Watson, Greatbatch and Parker, 1998, Giacomini and Cook, 2000, Mays and Pope, 1995, Mays and Pope, 1997, Mays and Pope, 2000). There are a number of essential requirements for successful qualitative research. Participant selection must be well reasoned, and relevant to the research question. Data collection processes should be appropriate for the setting and the research objectives, and comprehensive enough to support rich and robust descriptions of observed events (it includes such methods as document analysis, interviews and field observation). Finally, data must be appropriately analysed; findings should be corroborated by the use of multiple sources (triangulation), more than one investigator should collect and analyse the raw data, and researchers should check each other's 
interpretation to establish whether the participants' viewpoints were fairly and adequately represented.

Triangulation is the most frequently cited technique for counteracting threats to the plausibility of natural field research (Burgess, 1984, Delamont, 1992, Denzin, 1970, Hammersley and Atkinson, 1983). The process involves two or more 'sightings' of a phenomenon (finding), each 'sighting' taken from a different 'fixed' observation point. The use of multiple approaches to obtain research findings therefore avoids the bias of single approaches. The researcher takes observations from as many different angles as possible. Four different types of triangulation were identified by Denzin (1970): data triangulation, methodological triangulation, theory triangulation, and investigator triangulation. The first two are relevant to this study. Hammersley and Atkinson (1983) note that data source triangulation 'involves the comparison of data relating to the same phenomenon but deriving from different phases of the fieldwork, different points in the temporal cycles occurring in the setting...[and] the accounts of different participants... involved in the setting' (p.198).

The present study involved the collection of data across times (for example, before and after the introduction of the DHB health reforms, in different places (for example, health authorities, Ministry of Health, DHBs) and from different people (for example, managers, researchers, public health physicians, Planning and Funding Managers). Data triangulation thus provides the researcher with information that allows comparison and contrast between phenomena from diverse data sources: 'If diverse kinds of data lead to the same conclusion, one can be more confident in that conclusion.' (Hammersley and Atkinson, 1983, p.198). One method of triangulation alone is deemed insufficient as 'no individual method alone [can] yield the truth about a situation' (Burgess, 1984, p.154). In addition, to enhance credibility, it is recommended that multiple methods are pursued to examine a phenomenon or situation (that is, 'between method' triangulation): 'The rationale for this strategy is that the flaws of one method are often the strengths of another, and by combining methods, observers can achieve the best of each, while overcoming their unique deficiencies.' (Denzin, 1970, p.308). 
Interviews, document analysis, and case studies were the predominant methods used in the present study. By examining data related to the same phenomena from a combination of these methods, the researcher was able to check the credibility of inferences and findings. This process involves constant comparative checking to provide validity for data analysis. Overall, the research used a qualitative approach, supported by quantitative methods where the data permitted.

Documents are a rich source of data for social research. Document analysis of public papers, agenda papers, minutes, internal documents, correspondence and other materials can provide useful qualitative data (Hodder, 1994, p.393-402 ). It is especially useful in health policy research and programme evaluation. Different approaches may be taken to the analysis of documents. Some studies depend entirely on documentary data, with such data the focus in their own right. But as Punch states:

In other research, for example case studies or grounded theory studies, documentary data may be collected in conjunction with interviews and observations. In conjunction with other data, documents can be important in triangulation, where an intersecting set of different methods and data types is used in a single project' (Punch, 1998, p.190).

Document analysis was an important qualitative method used in this research, as it allowed collection of data that was otherwise unavailable. It allowed the obtaining of primary information regarding the key priorities established by DHBs, through analysis of DSPs and DAPs. These documents also form an important resource base for information regarding the planning activities of DHBs. Other documents used in the research (for example, Ministry of Health policy statements, DHB operational policy frameworks) gave additional background information that would have otherwise been difficult to collect.

Documents are a rich source of data for policy research. Jupp (1996, p.303) suggests four key questions in evaluating documentary data: its authenticity (whether it is original and genuine), its credibility (whether it is accurate), its representativeness (whether it is representative of the totality of the documents of its class), and its meaning (what it intended to say). Sampling bias during the present research was 
avoided by collecting and analysing the HNAs, DSPs, and DAPs for all of the 20 DHBs studied (noting that the 21 st DHB was not included in the study as it did not participate in the Health Reforms 2001 research project). These documents, published by DHBs were authentic, credible, representative (full collection), and legal documents that were assumed to have meaning. Other documents analysed during the course of the research included policy documents published by government departments, particularly the Ministry of Health. The possible sources of bias outlined above are countered by the reliable sources of the documents, and the completeness of sampling.

The interview is one of the main ways of collecting qualitative data. It is a very good way of accessing people's perceptions, meanings, definitions of situations and constructions of reality. As Jones puts it:

In order to understand other persons' constructions of reality, we would do well to ask them... and to ask them in such a way that they can tell us in their terms (rather than those imposed rigidly and a priori by ourselves) and in a depth which addresses the rich context that is the substance of their meanings. (Jones, 1985, p.46)

Interviewing in the context of qualitative research has a number of facets, which have been described as follows:

Interviewing has a wide variety of forms and a multiplicity of uses. The most common type of interviewing is individual, face-to-face verbal exchange, but it can also take the form of face-to-face group interviewing, mailed or self-administered questionnaires, and telephone surveys. Interviewing can be structured, semi structured or unstructured. It can be used for marketing purposes, to gather political opinions, for therapeutic reasons, or to produce data for academic analysis. It can be used for the purpose of measurement or its scope can be the understanding of an individual or a group perspective. (Fontana and Frey, 1994, p.361)

Regarding structured interviews, 'interview questions are planned and standardised in advance, pre-coded categories are used for responses, and the interview itself does not attempt to go to any great depth' (Punch, 1998, p.175). Unstructured interviews are open-ended and questions are not pre-planned, instead there are general questions to get the interview going, and to keep it moving. 'Specific questions will then emerge as the interview unfolds, and the wording of those questions will depend upon directions the 
interview takes' (p.176). Semi-structured interviews are in the middle of this spectrum usually there are pre-planned questions, but the interview is allowed to flow freely, with prompting allowed so as to obtain some specific information that may be sought. The interviewer gives some guide to the direction of the interview, but without being too intrusive.

In this research, a semi-structured interview approach was used to gather some specific information that was common to all interviewees, yet to allow them to express their ideas and perceptions freely within the broad dimension of the subject. Structured interviews 'would have allowed little room for response' (p.176), but unstructured interviews would not have allowed for 'pre-established categories for responding' ( $p$. 176). The semi-structured interview was therefore the most suitable for this research where certain information was required, yet an open-ended approach was required to allow other information to be freely offered.

An inductive approach was used for the analysis of key informant interviews. The inductive approach involves moving from observations/data towards generalisations, hypotheses, or theory, and is used in many types of qualitative analysis, especially grounded theory (Patton, 1990). In grounded theory, the analysis of the data is dependent solely on inductive interpretations of the raw data, using the subjects' own categories, concepts etc. It offers a systematic set of procedures for analysing data where the analysis is guided by specific objectives. It is the opposite of deduction, which is the process of data gathering to test predefined theory or hypotheses. A semistructured interview guide, incorporating the objectives, was designed for each set of qualitative interviews, and used to ensure that all question areas are covered uniformly for all participants. In addition, multiple probe questions were developed for each question, to obtain in-depth information regarding the area under investigation.

Case-studies are also used in this research. A case may be simple or complex, but with Miles and Huberman (1994) a case can be defined as a phenomenon of some sort occurring in a bounded context. A case may be an individual, or a role, or a small group, 
or an organisation, or a community, or a nation. It can also be a decision, policy, process, incident or event. Brewer and Hunter (1989) list six types of units that may be studied in social science research: individuals; attributes of individuals; actions and interactions; residues and artefacts of behaviour; settings, incidents, and events; and collectivities. Any of these may be the focus of case study research.

Yin stressed that a case study is an empirical inquiry that:

Investigates a contemporary phenomenon within its real-life context when:

- The boundaries between phenomenon and context are not clearly evident, and in which,

- Multiple sources of evidence are used. (Yin, 1984, p.23)

Stake gives a 'pretty loose definition' where he describes a case study as 'a study of a bounded system, emphasising the unity and wholeness of that system, but confining attention to those aspects that are relevant to the research problem at the time' (Stake, 1988, p.258).

In the present research, case studies are used in order to find common themes occurring within them, from which to draw common conclusions. These are a collective type of case study, involving multiple cases, with the focus within and across cases. Two groups of DHBs are chosen, one consisting of those demonstrating high performance, and the other consisting of those demonstrating low performance, regarding the planning processes used by them for their DSPs and DAPs. Case studies within each group are studied for common themes, looking to put forward common concepts, themes, or actions taken by those DHBs, in order to develop generalisations applicable to the group as a whole regarding their planning processes. From these understandings hypotheses are formed as to why different DHBs performed differently. These DHB case studies underline the potential generalisability of knowledge built from case studies. By using multiple case studies within the two broad groups chosen, it has been possible to obtain themes that were common to each group, and therefore obtain generalisable conclusions. Where that has not been possible, it has been clearly stated. 
The following table summarises the various methods used for each part of the research, drawing on the qualitative methods described above, as well as quantitative methods.

Table 10: Research methods used for each part of the evaluation programme.

\begin{tabular}{|l|l|l|l|}
\hline & \multicolumn{2}{|c|}{ Research methods } \\
\hline & $\begin{array}{l}\text { Document } \\
\text { analysis } \\
\text { Qualitative } \\
\text { interviews }\end{array}$ & $\begin{array}{l}\text { Quantitative } \\
\text { data }\end{array}$ \\
\hline Part 2: Past needs assessments. & $\bullet$ & $\bullet$ \\
\hline $\begin{array}{l}\text { Part 3: DHB health needs assessments, } \\
\text { prioritisation and impact on planning and } \\
\text { purchasing. }\end{array}$ & & \\
\hline $\begin{array}{l}\text { Case study DHBs } \\
\text { Part 4: Influence of Government policy and } \\
\text { contextual information. }\end{array}$ & $\bullet$ \\
\hline
\end{tabular}

\subsection{Research Design}

This research evaluates the impact of DHB HNA and prioritisation on the planning and purchasing process. This evaluation is a policy evaluation, the methods of which bear close relationship to the methods of programme evaluation. The aims of the research were as follows:

Part 1. To systematically review the international literature on need, theoretical and disciplinary approaches to HNAs, prioritisation, and the relationship between health services research and policy.

Part 2. To analyse health needs assessments conducted by the Ministry of Health, HFA and its predecessors between 1991 and 2000.

The objectives of this part of the study are to:

(a) Identify and catalogue health needs assessment work undertaken by the Ministry of Health, Public Health Commission and health authorities throughout New Zealand between 1991 and 2000. 
(b) Evaluate the impact of health needs assessments on service delivery, decision-making and policy.

(c) Review the overall impact of past health needs assessments (1991-2000).

Part 3. To evaluate health needs assessments and prioritisation undertaken by the DHBs.

The objectives of this part of the study are to:

(a) Evaluate the impact of DHB health needs assessments and prioritisation on health service planning and purchasing.

(b) Evaluate five case study DHBs regarding (a) above.

Part 4. Evaluate the relevance and effectiveness of government policy with respect to health needs assessment, prioritisation, planning and health service purchasing in the context of recent health reforms.

The overall research design was divided into four parts, with each part representing one of the aims above. The organisation of the various parts of the research is shown in the figure below: 


\section{Figure 9: Research Design and Organisation}

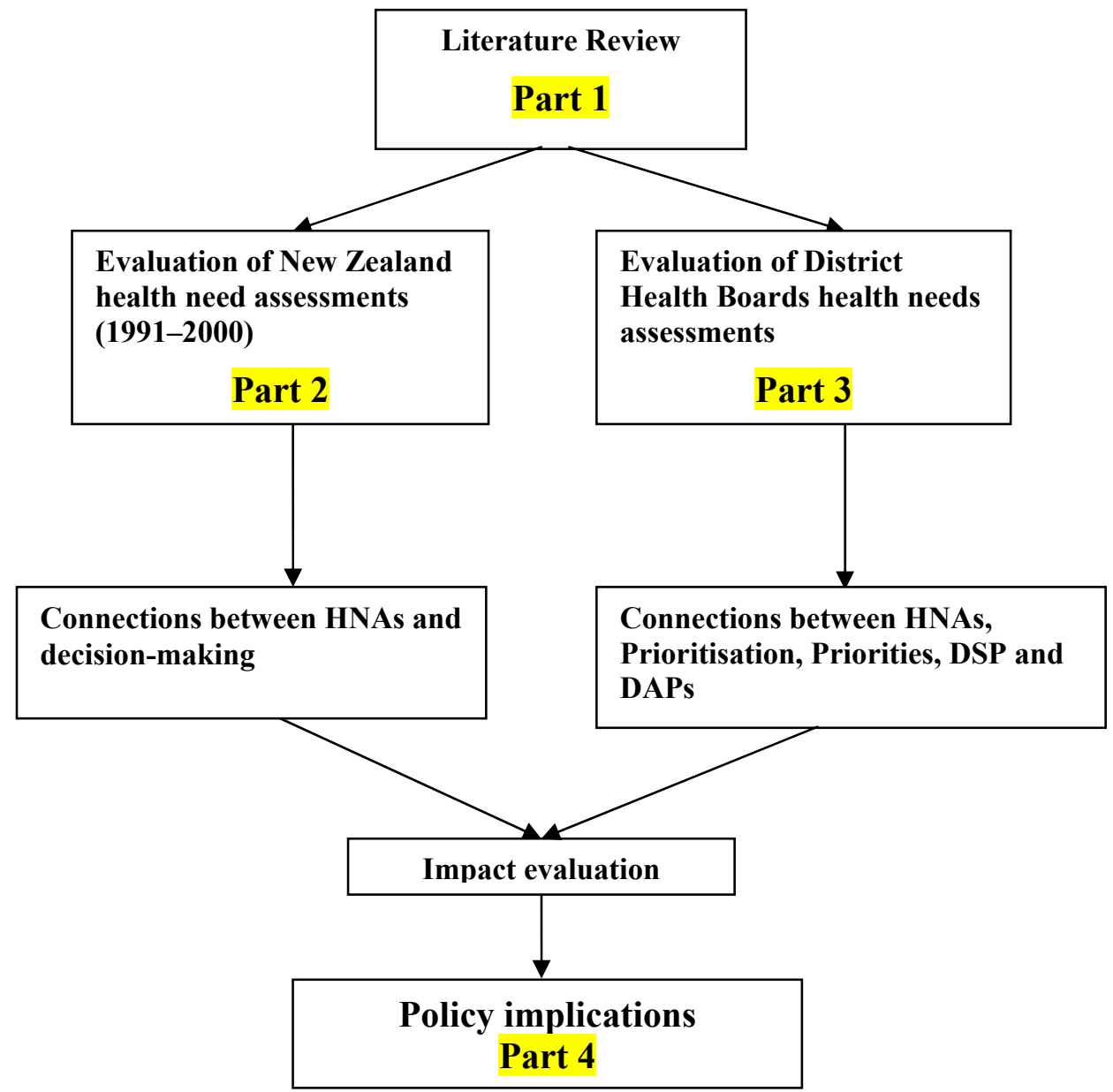

The four parts are now described in detail:

\subsubsection{Part 1. Literature review}

The public policy, health services research, social science, medicine, public health, general practice and primary health care literature was reviewed regarding need, HNA, prioritisation, and the relationship between health services research and policy. Searches were conducted using Medline, EMBASE, and PubMed using such key words and phrases as need, health care, health need, health care needs assessment, health needs assessment, demand, Māori health need, prioritisation, and health planning. References were also obtained from references. Relevant books were searched for references, as were Ministry of Health publications over the last ten years. This resulted in a considerable number of references, many of which appear in the Literature Review (see 
Chapter 2), but also elsewhere in the thesis, particularly in the Typology (see Chapter 3), policy context (see Chapter 4) and several of the discussion sections.

\subsubsection{Part 2. Evaluation of past health needs assessments (1991-2000)}

This included (a) health needs assessments undertaken by the Ministry of Health, Public Health Commission and Health Authorities in New Zealand between 1991 and 2000; (b) the impact of health needs assessments on service delivery, decision-making and policy; (c) the overall effectiveness of past health needs assessments.

Document analysis: A stocktake was undertaken of HNAs conducted in New Zealand from 1991-2000 by the Ministry of Health, Public Health Commission, Regional Health Authorities and the Health Funding Authority, and a bibliography of known needs assessments compiled. The following criteria used to select documents for the bibliography were based on those of Hensher and Fulop (1999), to allow comparison later with UK findings:

- It should be a 'population needs assessment' in that it explicitly used the word(s) need(s), wants or expectations in the title; and/or it contained a substantial element of analysis of local health or health care needs using epidemiological, demographic, qualitative, geographic, economic or community assessment (such as service reviews); and/or it reported or analysed local demographic, socio-economic or epidemiological data in the context of health, health status, or requirements for health care. (The word substantial is taken to mean more than half of the document; the terms referred to in this requirement are explained in Table 10); and

- it was undertaken by or on behalf of the Ministry of Health, a health authority or its predecessors. Needs assessments conducted by hospitals (Crown Health Enterprises, Hospital and Health Services) were specifically excluded on the grounds that they played a provider role, rather than that of funder; and

- it was completed between 1991 and 2000. 
Databases in the libraries of the Ministry of Health, Health Funding Authority and Locality Offices were searched to find HNAs that met the criteria for inclusion in the research. In order to widen the search, HNA reports were scanned for references to other HNAs. Health authorities were asked for copies of HNAs that met the criteria listed. People currently and formerly employed by health authorities were asked for advice regarding documents with which they had some involvement or for information regarding other documents. Informal networks were used to widen the range of contacts and information. High priority was given to accumulating these documents as knowledge regarding the existence and whereabouts of documents would potentially be lost following health restructuring under way at the time.

HNAs were then classified as population-based, community-based, epidemiological, comparative, corporate or economic, according to the typology presented in Chapter 3. Table 11 below shows the criteria that were used to derive the typology.

A table of HNAs was constructed, showing the HNA's title, service area, classification, author and year (see Appendix 3, p.298). The publisher or the report itself identified the sponsoring body of each assessment. The stated objectives of the HNAs were recorded, usually from forewords or introductions, sometimes from executive summaries, or terms of reference. The stated objectives were then classified according to their purpose, depending on whether they were related to service-specific purchasing decisions, measurement for research purposes (that is, to fill a gap in knowledge), community involvement, statutory consultation or some other primary objective. These results were then tabulated. Reports were also examined for recommendations made to agencies such as health authorities and purchasers. 
Table 11: Typological classification of health needs assessments

\begin{tabular}{|l|l|}
\hline Classification & Description \\
\hline $\begin{array}{l}\text { Population-based (also } \\
\text { known as global) }\end{array}$ & Assesses overall health care need in a large population or district \\
\hline Community-based & $\begin{array}{l}\text { Most 'community-based needs assessments' incorporate a high level of user and } \\
\text { community involvement, and } \\
\text { - are concerned with health services or general social and environmental issues that } \\
\text { affect health }\end{array}$ \\
\hline $\begin{array}{l}\text { - examine small areas or small population groupings } \\
\text { - involve work in the field } \\
\text { - adopt a flexible approach } \\
\text { - use largely qualitative data derived from the perspectives of the local community. } \\
\text { (Ong, Humphris, Annett and Rifkin, 1991) }\end{array}$ \\
\hline Epidemiological & $\begin{array}{l}\text { Needs are described in terms of disease rather than population groups or services. } \\
\text { Descriptive epidemiology (as opposed to analytical epidemiology) is used to } \\
\text { describe the occurrence of disease in terms of person, place, and time. }\end{array}$ \\
\hline Economic & \begin{tabular}{l} 
Services received by the population in one area are compared with those elsewhere. \\
\hline Comparative
\end{tabular} \\
\hline $\begin{array}{l}\text { Based on the demands, wishes and perspectives of interested parties, including } \\
\text { political and public views; involves collection of health data on health services from } \\
\text { key informants including staff of health authorities, provider clinicians, general } \\
\text { practitioners and consumers of health services. }\end{array}$ \\
\hline $\begin{array}{l}\text { Matches information on the costs of health care interventions to the benefits } \\
\text { produced so that purchasers can gain the greatest benefit from a defined budget. }\end{array}$ \\
\hline Corporate
\end{tabular}

Key informant interviews (health needs assessments 1997-2000): A similar methodology was used to that of Fulop (1997) who surveyed HNAs conducted in 14 London health authorities over a four-year period. Semi-structured interviews were undertaken with at least two representatives of the Health Funding Authority or the Ministry of Health who were with the relevant authority at the time and associated with the conduct of the assessment. One of the chosen interviewees was usually the project manager for the assessment in question, and the other a researcher or public health physician associated with the project.

Ethics Committee approval was obtained in advance from the Victoria University of Wellington Ethics Committee (see Appendix 4, p.303). This approval was specific to the research on the HNAs conducted between 1991 and 2000; further approval was later 
obtained for the DHB HNA research as part of the Health Reforms 2001 research. All interviewees were sent an invitation letter, information sheet and consent form prior to the interview, and written consent was obtained (see Appendices 5-7, p.304).

Twenty-five HNAs related to 1997-2000 were found to meet the criteria for inclusion in the study. Two interviewees for each HNA were invited by telephone or email to participate in the study. If the person chosen was not the most appropriate in the eyes of the health authority staff, their suggestions were followed up until the most suitable person(s) was found. Those approached were willing, with just one exception, to be interviewed. Some interviewees had been involved in several projects, and were willing to be interviewed regarding all of them. As a result, a potential field of 50 interviewees was reduced to 34 , and each was interviewed for up to one hour. The roles of the interviewees (managers or researchers) and the number of HNAs that they each conducted are reported in Chapter 6.

Interviewees were also asked to identify any other assessments that had been conducted by their organisation during the study period. Reports found in this way were added to the study to obtain as complete a record of HNA activity as possible.

The recruited interviewees each gave informed consent to a semi-structured interview by telephone. Interviews were tape-recorded with the interviewees' consent, and transcribed for analysis. Interviewees expressed their own personal views, rather than speaking on behalf of the institutions that employed them at the time or currently. Some interviewees had been independent contractors at the time of the needs assessments. Significantly, more than $85 \%$ (29/34) of those interviewed had changed employment since the needs assessments were completed.

The semi-structured guide for the interviews was developed using the literature on HNA as a resource (see Appendix 8, p.310). Questions were peer reviewed by two other researchers, and by a current DHB Planning and Funding manager. The draft questions were modified to take account of their feedback. Supported by the interview guide, the 
interviews were flexible 'guided conversations,' giving priority to informants' own accounts and interpretations. Informants were encouraged with prompts to express their views and insights regarding the topics under discussion.

Interviewees were asked to state why the project had been undertaken. Prompts included questions regarding statutory requirement, commissioned work, annual publication, follow up on national policy, pressure exerted on sponsoring body, regional or national initiative, special interest by an individual, routinely collected data, assisting purchasing decisions, research purposes, and any other reason.

Actions resulting from the project were tested with probing questions regarding qualitative or quantitative changes to existing services, service reviews with or without changes, other research required or actions taken as a result, whether recommendations of the report were implemented, success factors, and barriers. These were followed with questions regarding the way the project influenced purchasing decisions. They were asked to describe the actions resulting from the needs assessment, and to rate their perceptions of the extent to which the assessment had contributed to the purchasing decisions that they were endeavouring to make. They were also asked what other factors influenced the decisions. They were then asked whether community consultation took place, the extent of that consultation, and whether it included Māori.

Tables were drawn to show the stated objectives of HNAs (1997-2000), comparing 1991-2000 data with that of the London Health Authorities. The selection of topics for analysis by HNA was examined and also compared with the London Health Authority research data. Finally, the relationship between the method by which a topic was selected for detailed HNA and the subsequent impact of the needs assessment on decision-making (1997-2000) was also compared with the equivalent London Health Authority data.

Typed transcriptions of the audio recordings were read several times, systematically analysing and interpreting the text, searching for recurring themes and sub-themes 
using a grounded theory inductive approach to thematic analysis. In addition, the author systematically identified recurring themes arising from the literature that were then added to the master coding list. Finally text within the interviews was systematically coded using the master coding list, thereby incorporating both inductive and deductive approaches. This modification of a purist approach to grounded theory is common (Miles, 1979). This approach was taken in order to allow the opportunity for themes to arise inductively from the interview material, yet ensure for comparison purposes that the findings related to the concepts within the existing literature.

The author used NVivo software to aid data management by labelling text with an identified theme(s), or sub-themes. Some 54 coded themes and sub-themes were identified. Salient themes were established by collapsing themes in order to allow reporting of the large volume of material and generalisation to an appropriate theory.

The overall effectiveness of the historical HNAs was reviewed, taking into account the approaches used, the interpretation of data and their effectiveness in terms of influencing policy and implementation of new health services. These results from Part 2 of the research are reported in Chapter 6.

\subsubsection{Part 3. Evaluation of health needs assessments and prioritisation undertaken by the DHBs}

This included (a) health needs assessments; (b) prioritisation frameworks; (c) priorities established by DHBs; (d) the impact of health needs assessments and prioritisation on planning and purchasing; (e) five case study DHBs.

The research evaluation plan contained the performance measures necessary to answer the important questions concerning how well the policy was working to achieve its short term, intermediate and impact outcomes, recognising the external influences on the policy. A measurement plan cannot of necessity measure all aspects of a policy, and therefore some selection was made from identified performance measures in order to 
conserve evaluation resources. The following methods were chosen to answer the research questions posed.

Document analysis (health needs assessment): DHBs were contacted by letter and asked to supply copies of the HNAs conducted by Transitional DHBs before elected boards took office in December 2001. All the DHBs provided hard-copy documents, and many pointed to electronic versions available on their websites. Those DHBs that involved the Public Health Consultancy of the Wellington Clinical School in their HNAs also provided an additional summary document, together with a CD containing summary and technical reports. The Ministry of Health timetable required HNAs to be submitted by 1 November 2001. DHB Crown Funding Agreements for the 2001/02 year included an accountability indicator requiring effective HNA.

\section{Box 2: DHB Accountability Indicator GOV-02: Effective Health Needs Assessment}

DHB Accountability Indicator GOV-02: Effective Health Needs Assessment

A comprehensive health needs assessment report is produced for the DHB population

Associated deliverable

A Health Needs Assessment report (which covers the first two sections of the document Health Needs Assessment for New Zealand: An Overview and Guide, December 2000) is completed by 1 November 2001 for the DHB population which:

- Is consistent with the approach in Health Needs Assessment for New Zealand: An Overview and Guide, December 2000

- $\quad$ Gives particular attention to the New Zealand Health Strategy Population Health Priorities

- Analyses the distribution of diseases, environmental factors and their determinants across their population to identify those groups (which may include Māori, Pacific people and people in lower socio-economic groups) experiencing poorer health outcomes

- Lists the providers, services and numbers of patients receiving services from those providers again giving particular attention to New Zealand Health Strategy priorities

- Considers a range of evidence informed interventions including public health measures, actions on the wider determinants of health and health care services

- Involves participation and appropriate targeted consultation with groups within populations they are responsible for including local Iwi/Māori and where appropriate Pacific communities in respect to the Health Needs Assessment process

- Involves mainstream services provided to Māori (and where appropriate Pacific communities) and services provided by Māori health providers (and where appropriate Pacific providers) are captured in the Health Needs Assessment. (Ministry of Health, 2001a)

DHB health needs assessments were evaluated against this 'gold standard'. A letter was also sent to the Ministry of Health requesting information under the Official Information 
Act 1982 about health needs assessments and any evaluations the Ministry made of them.

Key informant interviews: Key informant interviews were conducted with 20 DHB Planning and Funding Managers to obtain their opinions regarding their HNA, prioritisation, and planning processes. Jackie Cumming, Principal Investigator for the Health Reforms 2001 Research project, sent a letter to the Chair and CEO of all Boards regarding the research. Ethics approval for the project was obtained from the Wellington Human Ethics Committee, acting on behalf of all National Ethics Committees. A copy of the consent is held at the Health Services Research Centre at Victoria University of Wellington.

DHB Planning and Funding Managers were informed that the researcher was studying the health needs assessment and prioritisation process in the context of the strategic planning cycle and written consent was sought for an hour-long interview (see Appendices 9-11, p.311). A semi-structured interview guide was developed, and was peer-reviewed by members of the Health Reforms 2001 Research project team, and by a Planning and Funding manager, to ensure relevance and appropriateness. Feedback suggestions were incorporated into the revised final questionnaire (see Appendix 12, p.320). Interviews were conducted by telephone and taped with permission. Taped interviews were then transcribed and checked for accuracy by interviewees. Amendments were incorporated into revised transcripts. The confidentiality of interviews, documents and the identity of individual interviewees were guaranteed. Interview tapes were stored in a locked facility at the University of Auckland. The transcriptions of the audio recordings were read several times. Texts were analysed closely and systematically, taking the same inductive approach used on the pre-DHB needs assessments, and using the same software to create analytic categories.

Document analysis (prioritisation): DHBs were contacted by letter and each asked to supply a copy of the prioritisation framework agreed by their DHB. All DHBs provided hard-copy versions of documents, and again many pointed to electronic versions on 
their websites. The Ministry of Health required DHBs to prioritise, by 31 May 2002, the need for health services in their districts, taking account of the directions set by the New Zealand Health and Disability Strategies and the Minister's priorities, within the available service funding. Crown Funding Agreements for the 2001/02 year included an accountability indicator for prioritisation, shown in the box below.

\section{Box 3: DHB Accountability Indicator GOV-03: Prioritisation}

DHB Accountability Indicator GOV-03: Prioritisation

Prioritisation of the needs of the DHB community is undertaken in terms of the directions set by the New Zealand Health and Disability strategies within available service funding.

Associated deliverable:

- Undertake a prioritisation round. Identify a list of funding options including planned sources of funding (which may include reprioritisation of current baseline expenditure) and provide the Ministry with a one page summary of the results by 31 May 2002. Include with the summary documentation evidence that shows that the prioritisation measure was met. (Ministry of Health, 2001a)

The commentary provided to DHBs regarding the Prioritisation accountability indicator states that it is expected that Boards will:

- Collaborate with other DHBs in relation to regional and national services

- Use a principle-based framework, that links directly to the principles of the New Zealand Health Strategy

- Involve Māori (and reflect their needs and support capacity building) throughout the development and implementation of the prioritisation process

- Clearly document: Why decisions were made; Who the decision makers were; What the decision making process was; How the community was involved in the decision making process. (Ministry of Health, 2001a)

This research also considers DHB prioritisation frameworks and particularly compares the principles adopted by different DHBs, using a tabulated form of analysis. The research does not take into account the ongoing work of the Ministry of Health in its prioritisation workstream, which was established in late 2002, and was still not complete in early 2004. However it does include consideration of the priorities established by DHBs. The implementation of key health needs assessment recommendations by means of DHB prioritisation, District Strategic Plans (DSPs) and District Annual Plans (DAPs) is also evaluated. 
Document analysis (Impact Factors): Impact Factors measure the impact of HNAs on DHB planning and purchasing. To determine the impact of HNA on planning and purchasing, the researcher calculated an Impact Factor for each DHB, based on document analysis of the HNAs, DSPs and DAPs. The method of deriving Impact Factors is outlined below.

DHB

HNA Key recommendations Hlth of low socio-economic Maori health Avoidable hospitalisations Intersectoral action

Smoking

Alcohol \& drug

Primary care

Rural health

Primary/secondary interface Health information

Water quality

Immunisation rates

Motor vehicle injuries

Cancer

Diabetes

Cardiovascular disease

Child health

Oral health

Respiratory diseases

Disability services

Suicide

\begin{tabular}{|c|c|c|}
\hline Prioritisation & Level of importance in DSP & Level of importance in DAP \\
\hline 1=Key priority & 1=Performance measure & 1=Performance measure \\
\hline 2=Significant priority & 2=Planned initiative (defined) & 2=Planned initiative (defined) \\
\hline 3=Less important priority & 3=Planned (not defined) & 3=Planned (not defined) \\
\hline 4=Not mentioned & 4=Not mentioned & 4=Not mentioned \\
\hline 4 & 3 & 3 \\
\hline 1 & 1 & 1 \\
\hline 4 & 3 & 4 \\
\hline 4 & 2 & 2 \\
\hline 1 & 2 & 2 \\
\hline 2 & 3 & 1 \\
\hline 1 & 1 & 1 \\
\hline 4 & 3 & 3 \\
\hline 4 & 3 & 3 \\
\hline 1 & 3 & 3 \\
\hline 3 & 3 & 4 \\
\hline 2 & 1 & 3 \\
\hline 4 & 4 & 4 \\
\hline 1 & 3 & 3 \\
\hline 1 & 2 & 2 \\
\hline 1 & 2 & 2 \\
\hline 1 & 2 & 2 \\
\hline 1 & 3 & 2 \\
\hline 1 & 2 & 3 \\
\hline 1 & 1 & 1 \\
\hline 2 & 2 & 1 \\
\hline
\end{tabular}

Figure 10: Example of the progression of HNA key recommendations into DHB Plans

Note: The figure shows the progression of HNA recommendations from HNA to Board prioritisation and into the DSP and DAP. The level of importance ascribed by the DHB to each recommendation is shown at each step, by means of a score and colour code, assigned following document analysis.

HNA documents obtained from DHBs were searched for key HNA recommendations, which were generally found within the body of the report, or sometimes in the summary. A list of all key HNA recommendations was generated for each DHB and entered into an Excel spreadsheet. Each key recommendation was then examined to ascertain the level of priority assigned to it by the DHB Board. This information was generally found recorded in the front section of the DSP documents. Referring to the 
spreadsheets (see Figure 10 above), each health need identified as a key HNA recommendation was assigned a score based on the level of importance ascribed to it, in three contexts: its prioritisation by boards; its importance in DSP; and its importance in $D A P$. The importance of these health need priorities was then scored from 1 to 4 , depending on whether they were a key priority $=1$ (red); significant priority $=2$ (orange); less important priority $=3$ (yellow); or not mentioned $=4$ (green). DSPs were then examined to ascertain the level of importance assigned to these priorities in the Strategic Plan, with scores allocated from 1 to 4 , depending on whether there was a performance measure $=1$ (red); planned initiative, defined $=2$ (orange); planned initiative, not defined $=3$ (yellow); or not mentioned $=4$ (green). Finally, Annual Plans were examined to ascertain the level of importance ascribed to these priorities, with scores allocated from 1 to 4 , depending on whether there was a performance measure $=$ 1 (red); planned initiative, defined $=2$ (orange); planned initiative, not defined $=3$ (yellow); or not mentioned $=4$ (green). The results of these analyses were recorded on the Excel spreadsheet, by DHB (see Appendix 13, p.322). An Impact Factor for each DHB was then devised as a measure of the progress of key HNA recommendations, from prioritisation into DSPs and then into DAPs.

There were two options available for analysing the spreadsheet data in order to obtain Impact Factors. The first option involved ranking DHBs using an expert panel of observers, who would be asked to rate DHBs on the basis of inspection of the spreadsheet data, using the colour codings. They would assess whether key recommendations in the needs assessments had maintained importance and flowed through to DSP and DAP, by DHB. For example, a set of red bars straight across the page would indicate a high degree of connection. Conversely, a set of green bars would indicate poor connection, as would bars that traversed from red to green. Various other combinations would also need to be ranked, and overall rankings determined. The expert panel would subjectively rank DHBs, according to panel members' estimates of the effect that HNA recommendations made during the planning process. This option of employing a panel of experts to rank DHBs was discarded in favour of the second option, in view of the subjectivity of the ranking process. 
The second option was to adopt a mathematical approach to the analysis of the Excel spreadsheets. ${ }^{26}$ Reading across the row gave rise to a three-digit number (for example, smoking 122). Each three-digit number was assigned a Value to reflect the impact of the HNA recommendation on the DHB plan. A Value of five reflecting high impact was achieved when the sum of the digits within a three-digit number was 4 or less, and a low Value of one reflecting low impact when the sum was 10 or greater, with intermediate rules for intervening values. This system recognises that the highest Value (5) should be assigned to a line of red bars all the way across, or to minor variations of this. Lesser Values are assigned to lines showing lesser connections. The table used to aid assigning a Value to each line is shown below.

Table 12: Values assigned to three-digit numbers during analysis of impact of HNAs on planning, and associated rules.

\begin{tabular}{|c|c|c|}
\hline Value & Three-digit numbers & Rules \\
\hline $\begin{array}{c}5 \\
\text { Very good }\end{array}$ & $\begin{array}{llll}111 & 112 & 211 & 121\end{array}$ & Sum of digits is 4 or less \\
\hline $\begin{array}{c}4 \\
\text { Good }\end{array}$ & $221212122 \quad 222$ & Sum is 5 or 6 , no 3's or 4's \\
\hline $\begin{array}{c}3 \\
\text { Satisfactory }\end{array}$ & $\begin{array}{llllllllll}123 & 231 & 232 & 113 & 321 & 223 & 331 & 231 & 322 & 213 \\
133 & 132 & 311 & 131 & & & & & & \end{array}$ & Sum is 5,6 or 7, no 4's \\
\hline $\begin{array}{c}2 \\
\text { Poor }\end{array}$ & $\begin{array}{llllllllll}332 & 234 & 241 & 242 & 323 & 141 & 224 & 441 & 324 & 243 \\
333 & 233 & 431 & 432 & 124 & 143 & 144 & 134 & 114 & 421 \\
422 & 341 & & & & & & & & \end{array}$ & Sum is $6,7,8$ or 9 \\
\hline $\begin{array}{c}1 \\
\text { Very poor }\end{array}$ & $244 \quad 442 \quad 433 \quad 344 \quad 444 \quad 434 \quad 343$ & Sum 10 or greater \\
\hline
\end{tabular}

The Impact Factor for a DHB was obtained by averaging all the Values using the mathematical relationship:

$$
\Sigma\left(\mathrm{n}_{1} \mathrm{~V}_{1}+\mathrm{n}_{2} \mathrm{~V}_{2}+\mathrm{n}_{3} \mathrm{~V}_{3}+\mathrm{n}_{4} \mathrm{~V}_{4}+\mathrm{n}_{5} \mathrm{~V}_{5}\right)
$$

Impact Factor $=\quad \sum\left(\mathrm{n}_{1}+\mathrm{n}_{2}+\mathrm{n}_{3}+\mathrm{n}_{4}+\mathrm{n}_{5}\right)$

\footnotetext{
${ }^{26}$ I am indebted to Prof. David Wilkin of the National Primary Care Research and Development Centre, University of Manchester, for suggesting a mathematical approach. Several methods were trialled and one devised by myself was finally chosen.
} 
Where $\mathrm{n}=$ number of key recommendations (three-digit numbers)

$\mathrm{V}=$ value assigned to each three-digit number

Thus an Impact Factor was calculated for each DHB, relating to the impact of the HNA on prioritisation, DSP and DAP, using the document analysis approach described above.

A high Impact Factor meant that the DHB planning process resulted in the HNA having a significant impact on the DHB Strategic Plan and Annual Plan. Conversely, a low Impact Factor meant that the HNA had little impact on the planning process. Impact Factors could then be used for inter-DHB comparisons of the effectiveness or impact of HNA recommendations on the DHB planning process.

Key informant interviews (prioritisation): DHB Planning and Funding Managers were asked at interview how well connected or 'joined up' the following were: HNA and prioritisation; prioritisation and DSP; DSP and DAP; DAP and the budget; and budget and purchasing. Interviewees answered in their own words with replies varying from a short response to a more elaborate explanation. Information from this part of the interviews was used to develop a Connection Score, which was a measure of how well Planning and Funding Managers thought that various parts of the planning cycle were connected.

Interviews with DHB Planning and Funding Managers were analysed for the strength of connections between the key recommendations from HNAs and prioritisation by DHBs, and DSPs and DAPs. Transcripts of the interviews were read several times, taking particular note of the stated views of Planning and Funding Managers regarding the strength of connection between the various stages on the path from HNA to purchasing.

The managers' estimates of the strength of connection were then assigned a score on the following basis: strong connection $=3$; moderately strong connection $=2$; weak connection $=1$; and no connection $=0$. Individual scores were allocated for each of the interfaces between HNA and Board priorities; Board priorities and DSP; DSP and DAP; 
and DAP and purchasing. The transcripts were examined for evidence that interviewees were consistent in the opinions that they expressed. In those cases where information given elsewhere during the interview was at odds, the original score was carefully modified to reflect the overall evidence of the interview. Scores were then added together to obtain an overall Connection Score for the planning process of each DHB.

Information obtained from document analysis (Impact Factors) and key informant interviews (Connection Scores) were then compared regarding the impact of HNAs on prioritisation, DSPs, DAPs and purchasing, using a triangulation process. Regression analysis was used to test the relationship between Impact Factors, Connection Scores, Quality Scores (HNAs), and Board deficits.

Case study DHBs: Five case study DHBs were used to illustrate differences between those DHBs (examples A, B and C) whose HNA had the highest impact on purchasing, and two examples that were found to have less impact (D and E). The interviews for the five DHBs were scrutinised for information regarding the different ways that the planning process from HNA to DAP was managed, looking to find generalisable differences between the two groups of case study DHBs.

The activities undertaken by case study DHBs in HNA, service planning groups, prioritisation and consultation were researched. HNAs, DSPs and DAPs were also studied in depth in order to obtain an overall picture. By comparing the differences in approach between those boards that were more successful in the planning process with those that were less successful, it was possible to ascertain reasons for success, or not. The results from Part 3 of the research, including the case studies, are reported in Chapter 7. 


\subsubsection{Part 4. Evaluation of the effectiveness of government policy regarding health needs assessment, prioritisation, and planning}

Including (a) the context of recent health reforms and (b) relevance and effectiveness of government policy regarding HNA, prioritisation, and planning and purchasing in that context.

Key informant interviews: Two key informants from the Sector Policy Directorate of the Ministry of Health were interviewed (one after leaving the Ministry) regarding policies for HNA and prioritisation. Interviews covered both past and future HNAs, and consent was obtained in the usual way. Interviews were tape-recorded and transcribed as described above, and analysed in a similar manner to those with DHB Planning and Funding Managers. Transcripts were analysed for information regarding the Ministry's intentions for HNA and prioritisation.

Contextual data: A range of general documents was studied in order to understand the broader context of the health reforms regarding HNA and prioritisation and their impact on planning and service purchasing. Documents relating to reforms from 1983 until the present time were reviewed. The results from Part 4 of the research are discussed in Chapters 7 and 8.

\section{Research timeline}

The literature review commenced in early 2000, and was followed by the collection of HNAs conducted by health authorities between 1991 and 2000. The interviews with two Ministry officials were undertaken in late 2001. The 34 interviews with HNA managers and researchers and the analyses of those interviews were completed by early 2002 . Simultaneously, DHB HNAs and supporting material were being gathered, enabling interviews with the 20 DHB Planning and Funding Managers to commence by mid2002, with analysis continuing during the early part of 2003. The case study DHB information was collected and analysed during 2002-3. Meanwhile writing-up of the thesis was continuing, as was collection and analysis of contextual material. Overall, the 
timing of each part of the research ensured that components were undertaken in a rational sequence, which allowed the researcher to consider and interpret events as they occurred. The following Gantt Chart shows the timing relationships of the various components of the research.

Figure 11: Gantt Chart showing the timing relationships of the various components of the research

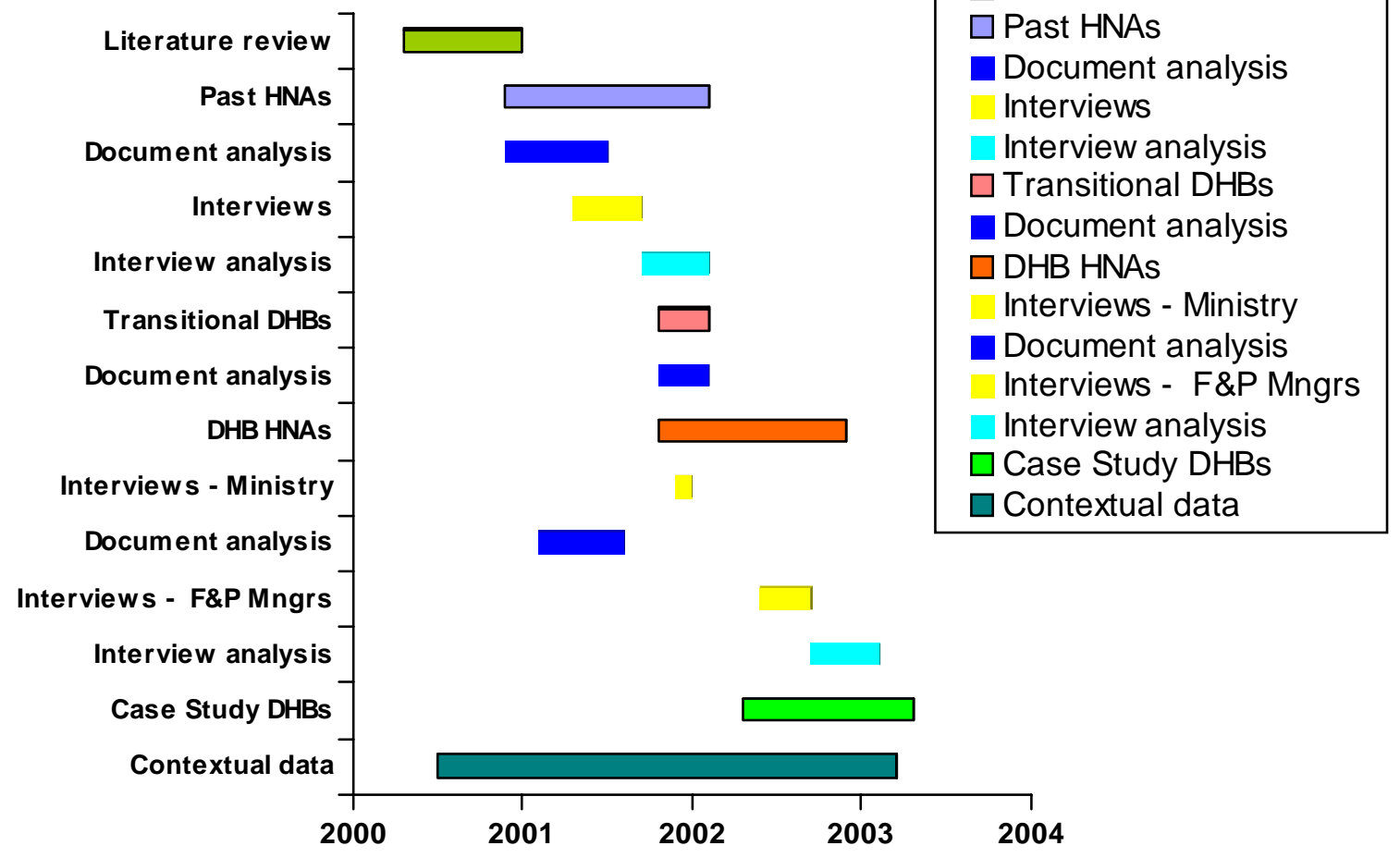


Chapter 6: Past Needs Assessments (1991-2000)

6.1 Introduction

6.2 Overview of HNAs 1991-2000

6.3 Overview of HNAs 1997-2000

6.4 Interview findings regarding HNAs 1997-2000

6.4.1 Planning and conduct of HNAs

6.4.2 Prioritisation

6.4.3 Purchasing decisions

6.4.4 Consultation

6.5 Discussion 


\subsection{Introduction}

The aim of Part 2 of the research was to evaluate the impact of HNAs conducted by the Ministry of Health, Public Health Commission and health authorities throughout New Zealand between 1991 and 2000 to inform decision-making regarding health service purchasing.

The objectives of this part of the study were to:

(a) Identify and catalogue HNA work undertaken by the Ministry of Health, Public Health Commission and health authorities throughout New Zealand between 1991 and 2000.

(b) Evaluate the impact of HNAs on service delivery, decision-making and policy.

(c) Review the overall impact of past HNAs (1991-2000).

HNAs conducted by health authorities prior to December 2000 have not been studied previously. It is useful to examine them to determine their effectiveness in influencing decision-making, service delivery, and health policy, and to consider their lessons for DHBs as health needs assessors.

The period chosen for this research, 1991-2000, covers two and a half years of the former Area Health Boards, and the duration of the RHAs and Health Funding Authority, and ends at 2000 before DHBs were created. The method employed for the research has been discussed earlier (see Chapter 5.3.2, p.133). The criteria for including reports in this study are as follows, and are based on that of Hensher and Fulop (1999), for comparison purposes:

- It should be a 'population needs assessment' in that it explicitly used the word(s) need(s), wants or expectations in the title; and/or it contained a substantial element of analysis of local health or health care needs using epidemiological, demographic, qualitative, geographic, economic or community assessment (e.g. service reviews); 
and/or it reported or analysed local demographic, socio-economic or epidemiological data in the context of health, health status, or requirements for health care. (The word substantial is taken to mean more than half of the document; the terms referred to in this requirement are defined in Table 11); and

- it was undertaken by or on behalf of the Ministry of Health, a health authority or its predecessors. Needs assessments conducted by hospitals (Crown Health Enterprises, Hospital and Health Services) were specifically excluded on the grounds that they played a provider role, rather than that of funder; and

- it was completed between 1991 and 2000.

\subsection{Overview of HNAs 1991-2000}

This section reports on the document analysis of HNAs completed during the period 1991-2000. More than one hundred and fifty New Zealand reports were canvassed for inclusion in the study. Fifty HNAs met the inclusion criteria stated in the methods chapter. Most of those reports omitted were policy statements regarding services to be purchased. Specified health authorities had not published the remainder. Some collections of reports collectively covering the whole of New Zealand were treated as single reports for the purposes of this analysis (for example, demographic and epidemiological profiles of various localities, published by the Health Funding Authority). This was done to avoid over-weighting the group with a large number of reports that were virtually the same.

Two further reports were identified as a result of the interviews. HNAs conducted during the decade 1991-2000 are listed in Appendix 3, p.298, as well as in the References. Table 13 below shows the classification of the HNAs (1991-2000), according to the typology presented earlier (see Chapter 3, p.68). 
Table 13: Classification of HNAs (1991-2000)

\begin{tabular}{|l|c|c|}
\hline Classification & $\begin{array}{c}\text { Number of studies } \\
(\mathbf{n = 5 0 )}\end{array}$ & Percentage of studies \\
\hline Population-based & 29 & $58 \%$ \\
\hline Community-based & 5 & $10 \%$ \\
\hline Epidemiological & 6 & $12 \%$ \\
\hline Comparative & 4 & $8 \%$ \\
\hline Corporate & 6 & $12 \%$ \\
\hline Economic & 0 & $0 \%$ \\
\hline
\end{tabular}

The majority of the reports were population-based HNAs (58\%), with relatively low proportions of the other types. There were no economic HNAs. The predominance of population-based HNAs is not surprising given that HNA activity was new for most health authorities, and that no rational framework had been established, as for example by the NHS in the UK in 1991. The HNAs were analysed for their commissioning health authorities and the results are presented in Table 14.

Table 14: Sources of HNAs (1991-2000)

\begin{tabular}{|l|c|c|}
\hline Source & $\begin{array}{c}\text { Number of studies } \\
(\mathbf{n = 5 0 )}\end{array}$ & $\begin{array}{c}\text { Percentage of } \\
\text { studies }\end{array}$ \\
\hline Department of Health to 1993 & 2 & $4 \%$ \\
\hline Area Health Boards to 1993 & 3 & $6 \%$ \\
\hline Regional Health Authorities 1993-1997 & 17 & $34 \%$ \\
\hline Health Funding Authority 1997-2000 & 7 & $14 \%$ \\
\hline Public Health Commission 1993-1995 & 6 & $12 \%$ \\
\hline National Health Committee 1993-2000 & 2 & $4 \%$ \\
\hline Ministry of Health 1993-2000 & 13 & $26 \%$ \\
\hline
\end{tabular}

Note: The Ministry of Health was established on 1 July 1993, from the former Department of Health. 
The HNAs were analysed for their stated objectives, which are recorded in Table 15.

Table 15: Stated objectives of HNAs (1991-2000)

\begin{tabular}{|l|c|c|}
\hline Objective & $\begin{array}{c}\text { Number of studies } \\
(\mathbf{n = 5 0 )}\end{array}$ & $\begin{array}{c}\text { Percentage of } \\
\text { studies }\end{array}$ \\
\hline Service-specific purchasing decisions & 5 & $10 \%$ \\
\hline General purchasing decisions $^{2}$ & 10 & $20 \%$ \\
\hline Measurement for research purposes $^{3}$ & 13 & $26 \%$ \\
\hline Other primary objective $^{4}$ & 1 & $2 \%$ \\
\hline More than one of the above $^{5}$ & 18 & $36 \%$ \\
\hline Community involvement $^{6}$ & 1 & $2 \%$ \\
\hline Statutory consultation $^{7}$ & 2 & $4 \%$ \\
\hline
\end{tabular}

Key: 1. Reports commissioned to assist decision-making on the purchasing of specific services, for example, maternity; 2. Reports commissioned to assist decision-making regarding the purchasing of a wide range of health services for a population; 3. Measurement for the purposes of research, not for purchasing; 4. Reports commissioned to assist decision-making regarding a specific issue, for example, Health care needs assessment study: South Island West Coast; 5. Reports commissioned to assist decision-making for more than one of the above reasons; 6. Reports commissioned to assist decisionmaking specifically by obtaining the involvement of the community; 7. Reports commissioned because they were required by Statute.

Eighteen reports listed in Table 16 recorded objectives that fitted the "more than one of the above' category, and the objectives of these reports were analysed further:

Table 16: Further breakdown of multiple objectives (refers to 'more than one of the above')

\begin{tabular}{|l|c|}
\hline Objectives & Number of studies $(\mathbf{n}=\mathbf{1 8})$ \\
\hline $\begin{array}{l}\text { Research and general purchasing decisions } \\
\text { desearch, general purchasing decisions and policy }\end{array}$ & 4 \\
\hline Research and other primary objectives & 5 \\
\hline Research and service-specific purchasing decisions & 1 \\
\hline $\begin{array}{l}\text { Research, service-specific purchasing decisions and policy } \\
\text { development }\end{array}$ & 5 \\
\hline $\begin{array}{l}\text { Service-specific purchasing decisions and policy } \\
\text { development }\end{array}$ & 1 \\
\hline
\end{tabular}

Note: The categories of objectives used in this table are those used in Table 16 above, but grouped.

Research was an objective of 17 of the 18 reports in the group. Furthermore, in over half of these reports, policy development was also an objective. Overall, purchasing decisions were the key objective in $30 \%$ of the needs assessments, but this proportion increased to $54 \%$ when reports that had mixed objectives were included. A very low proportion of the reports $(2 \%)$ resulted from a decision by the health authority to seek 
more community involvement in decision-making. The reports were analysed for the agencies to which recommendations were made and the results shown in Table 17.

Table 17: Recommendations made to agencies (1991-2000)

\begin{tabular}{|l|c|c|}
\hline Recommendations made to: & Number (n=50) & $\begin{array}{l}\text { Percentage of } \\
\text { studies }\end{array}$ \\
\hline Ministry of Health & 4 & $8 \%$ \\
\hline Health authority & 15 & $30 \%$ \\
\hline Ministry of Health and health authority & 3 & $6 \%$ \\
\hline $\begin{array}{l}\text { Ministry of Health, health authority, and other } \\
\text { agencies }\end{array}$ & 3 & $6 \%$ \\
\hline National Health Committee and health authority & 2 & $4 \%$ \\
\hline Not clear to whom recommendations apply & 2 & $4 \%$ \\
\hline No recommendations made & 21 & $42 \%$ \\
\hline
\end{tabular}

It would normally be expected that needs assessment exercises would carry recommendations for a health authority or purchaser. Therefore it is surprising that $42 \%$ of reports make no recommendations.

\subsection{Overview of HNAs 1997-2000}

Twenty-five documented studies for the years 1997-2000 were found that met the inclusion criteria. The reports from those four years were classified as follows, using the typology in Chapter 3:

Table 18: Classification of HNAs (1997-2000)

\begin{tabular}{|l|c|c|}
\hline Classification & $\begin{array}{c}\text { Number of studies } \\
(\mathbf{n = 2 5})\end{array}$ & Percentage of studies \\
\hline Population-based & 18 & $72 \%$ \\
\hline Community-based & 1 & $4 \%$ \\
\hline Epidemiological & 1 & $4 \%$ \\
\hline Comparative & 1 & $4 \%$ \\
\hline Corporate & 4 & $16 \%$ \\
\hline Economic & 0 & $0 \%$ \\
\hline
\end{tabular}

Note: Studies are classified by the main approach used.

The distribution of types of report is roughly similar to that over the last decade, with by far the commonest type being the population-based HNAs. This is not altogether 
surprising given their usefulness from a broad purchasing perspective, and that HNA was a relatively new experience for New Zealand. They are also more likely to be the type of report of interest to public health physicians. Twelve reports (48\%) published between 1997 and 2000 contain no recommendations, which is similar to the proportion $(42 \%)$ for the whole decade. There appeared to be general reluctance by health need assessors to make recommendations on the strength of HNAs. This seems remarkable given that assessors were almost certainly in the best position to make those recommendations and that HNAs are essentially practical exercises, not research per se.

It is possible that some of the reasons for conducting HNAs were not recorded in the reports. Such reasons could include gathering information to support purchasing decisions already made, but yet to be made public, and political considerations.

The 25 past HNAs (1997-2000) were divided into three broad groups: (a) HNAs that were predominantly epidemiological exercises; (b) service-specific HNAs; and (c) HNAs with both epidemiological and community data. These groups are now discussed in turn.

\section{(a) HNAs that were predominantly epidemiological exercises}

The following reports, containing mainly epidemiological data, were included in this group: ${ }^{27}$

- Our health our future = hauora pakari, koiora roa: the health of New Zealanders, 1999.

- Taking the pulse: The 1996/97 New Zealand Health Survey.

- Disability in New Zealand - Overview of the 1996/97 Surveys.

- Our children's health: key findings on the health of New Zealand children.

- Progress on health outcome targets, Te haere whakamua ki nga whainga hua mo te hauora, 1997.

${ }^{27}$ Refer to Appendix 3, p.298 for a full list of reports and references. 
- $\quad$ Progress on health outcome targets, Te haere whakamua ki nga whainga hua mo te hauora, 1998.

- $\quad$ The Northern Region Health Survey: 1996/97.

- Report to the Ministry of Health: Health Profile of the Wellington Region.

- The health of the people in the south: West Coast, Canterbury, Otago, Southland.

- Pacific Islands People in the North Health Region.

- Socio-economic inequalities in health care.

- $\quad$ Profile of the (Wellington) Locality.

This is the largest group of HNAs, which is not surprising given the relative ease of collating epidemiological data. They are all population-based and address the broad need for health services for identified populations. It could be argued that these reports are just health profiles, and therefore should not be considered HNAs in any practical sense. However, they all met the chosen definition of HNA and have therefore been included (see Chapter 5.3.2, p.133). The first four are Ministry of Health reports, and utilise information obtained from the New Zealand Health Survey. This large dataset is held by the Ministry of Health. They were not primarily undertaken for HNA purposes, and in some cases were conducted to meet statutory obligations. These reports are important, however, because they inform new health policy and are useful to help identify health service priority areas. The information is useful for such policy documents as the New Zealand Health Strategy (Minister of Health, 2000d) and the New Zealand Disability Strategy (Minister of Health, 2001b). For example, data from the New Zealand Health Survey provided background information to assist decisionmaking regarding the key population priority objectives for New Zealand that are set out in the New Zealand Health Strategy.

The Profile of the Wellington Locality is one of a larger set of 21 uniform reports, each covering a single DHB district within New Zealand. These reports were prepared before the establishment of the DHBs to provide them with data regarding their districts. Only one of the 21 reports was included in the 1991-2000 sample, as they were very similar. They are all listed in Appendix 3. One example of the use of these reports was in the production of the Counties Manukau DHB community health profile published in mid2001 (Jackson, Palmer, Lindsay and Peace, 2001). 


\section{(b) Service-specific HNAs}

The following reports were included in this group: ${ }^{28}$

- Family Health Services in the Midland Region.

- Sexual and Reproductive Health in the Midland Health Region, Volume 2, Part III.

- Pregnancy \& Childbirth in the Midland Region.

- Kia Tu Kia Puawai: Mental Health.

- Case study: Maternity Services and Care in Porirua.

- Review of maternity services in New Zealand.

- Korero Pasifica: Making a Pacific difference: strategic initiatives for the health of Pacific people in New Zealand.

- $\quad$ Aged Residential Care Utilisation in the Mid-North Island.

These HNAs were all service-specific in that they addressed the need for defined services for particular groups of people. Service-specific reports were more likely to make recommendations to purchasers. The reports generally used a combination of population-based and community-based approaches to HNA, as described in the typology in Chapter 3. There was a moderately heavy reliance on population demographic data as a basis for these reports, as well as extensive use of epidemiological data. However some of the HNAs (the first five reports) also included a significant amount of data derived from the community. Some reports contained a significant component of policy recommendations, particularly the last three. The first three reports were high quality in-depth studies from the Health and Disability Analysis Unit in the former Midland Health Authority. Community data from the Midland Health Community Health Survey were included (Health \& Disability Analysis Unit Midland Health, 1993). The Unit was highly skilled and had sufficient resources to support the research. All reports in this group contained significant evidence-based policy recommendations designed to improve health services. The Midland Unit was disbanded at the time of the formation of the Health Funding Authority, and it is the view of many interviewees that a significant resource was lost.

\footnotetext{
${ }^{28}$ Refer to Appendix 3, p.298 for a full list of reports and references.
} 


\section{c) HNAs with both epidemiological and community data}

The following reports were included in this group: ${ }^{29}$

- Kapiti District Health and Disability Report and Plan.

- Porirua City Health and Disability Report and Plan.

- Improving our Health in Wellington.

- Health Care Needs Assessment Study: South Island West Coast.

- Korero Pasifica: Consultation Review: Making a Pacific Difference.

Three of these five reports (excluding Health Care Needs Assessment: Study South Island West Coast Study; Korero Pasifica: Consultation Review Making a Pacific Difference) had a strong component of epidemiological and demographic data regarding their communities of interest. The data detailed in the reports were obtained from community provider focus groups and key informant interviews. However, none of the reports contained data obtained directly from individuals in the community by survey, interview, or other methods. Rather, they took a broad perspective over entire communities, and four of the five were global or population-based assessments. The remaining one (Korero Pasifica) had strong community involvement, using large community meetings to obtain input. All contained clear statements of community needs, but none had prioritised those needs.

The first two reports, conducted prior to the Ministry of Health setting out its policy for HNAs (Ministry of Health, 2000b), nearly met the current policy guidelines. They set out clear objectives and descriptions of their methods, used extensive epidemiological and demographic data, and consulted with provider and community groups by means of focus groups and interviews with individuals. They did not include any consumer survey data, and the consumer interviews were limited. But they should otherwise be regarded as the best of HNAs. They were both conducted immediately before the HNA policy was formulated, and involved Ministry of Health staff, and therefore it is not surprising that they were good.

${ }^{29}$ Refer to Appendix 3, p.298 for a full list of reports and references. 


\subsection{Interview findings regarding 1997-2000 HNAs}

\section{Introduction}

To obtain more in-depth information regarding recent HNAs, interviews were conducted with managers and researchers (often public health physicians) who had been associated with the HNAs conducted over the target period (1997-2000). This period was chosen as it antedated DHBs but was still recent enough to be fresh in the minds of interviewees. Also, it was difficult to locate the sponsors and authors of the reports previous to 1997, as a result of personnel changes in the health sector during recent reforms. Personnel changes affected all agencies including Regional Health Authorities, the Transitional Health Authority, the Health Funding Authority and the Ministry of Health. The design of the interviews is described in the methods section 5.3, p.130.

Interviewees were asked for their views on HNAs conducted between 1997 and 2000 in a semi-structured interview. ${ }^{30}$ Some managers and researchers had been involved in multiple HNAs for the period reviewed. This meant that 34 interviews were conducted to cover the 50 HNAs. The numbers of HNAs conducted by managers and researchers for 1997-2000 HNAs are shown in Table 19.

Table 19: Numbers of HNAs conducted by managers and researchers, for 1997-2000

\begin{tabular}{|l|c|c|}
\hline & Managers & Researchers \\
\hline One HNA & 10 & 11 \\
\hline Two HNAs & 6 & 5 \\
\hline Three HNAs & 1 & 0 \\
\hline Four HNAs & 0 & 1 \\
\hline Total HNAs & 25 & 25 \\
\hline
\end{tabular}

Apart from one researcher who was involved in four HNAs, there was an even distribution of involvement between managers and researchers. Notably, 11 researchers performed only one health need assessment each during the period, as did a similar

${ }^{30}$ Refer Appendix 10, p.313 for the semi-structured guide used for interviews with HNAs participants 1997-2000. 
number of managers. This table does not take account of the fact that some of the researchers involved also participated in other HNAs, but not as a lead assessor.

\section{Stated objectives of HNAs}

The stated objectives for conducting HNAs are listed in Table 20 below. Analysis showed that 12 needs assessments in total (48\%) were intended to assist decisionmaking for health service purchasing (purchasing decisions were involved for six in the category of 'more than one of the above'). This was a similar proportion to that of the reports of the entire decade (54\%). Fully one quarter were undertaken for research purposes alone.

Table 20: Stated objectives of HNAs (1997-2000), comparing 1991-2000 data, and LHA data.

\begin{tabular}{|l|c|c|c|c|}
\hline \multicolumn{1}{|c|}{ Objective } & $\begin{array}{c}\text { Number of } \\
\text { studies } \\
\text { 1997-2000 } \\
\text { (n=25) }\end{array}$ & $\begin{array}{c}\text { Percentage } \\
\text { of studies } \\
\mathbf{1 9 9 7 - 2 0 0 0}\end{array}$ & $\begin{array}{c}\text { Percentage } \\
\text { of studies } \\
\mathbf{1 9 9 1 - 2 0 0 0}\end{array}$ & $\begin{array}{c}\text { Percentage } \\
\text { of studies } \\
\text { LHA }\end{array}$ \\
\hline Service-specific purchasing decisions & 2 & $8 \%$ & $10 \%$ & $31.4 \%$ \\
\hline General purchasing decisions & 4 & $16 \%$ & $20 \%$ & $21.5 \%$ \\
\hline Measurement for research purposes & 6 & $24 \%$ & $26 \%$ & $14.1 \%$ \\
\hline Other primary objective & 0 & $0 \%$ & $2 \%$ & $12.0 \%$ \\
\hline More than one of the above & 11 & $44 \%$ & $36 \%$ & $9.4 \%$ \\
\hline Not stated, not clear & 0 & $0 \%$ & $0 \%$ & $7.3 \%$ \\
\hline Community involvement & 1 & $4 \%$ & $2 \%$ & $2.6 \%$ \\
\hline Statutory consultation & 1 & $4 \%$ & $4 \%$ & $1.6 \%$ \\
\hline
\end{tabular}

LHA, London health authorities. (Fulop and Hensher, 1997) Note: The categories used are those of Hensher and Fulop (1999), for comparison purposes. (See Table 15, p.153, for a description of the various categories).

\section{Selection of topics for health needs analysis}

Interviewees were asked to say why the project was undertaken, in order to ascertain how topics got on to the local or national agenda. They were given a range of categories from which to select. Similar responses were obtained from each pair of interviewees associated with the projects. The results are summarised in Table 21 . In $40 \%$ of cases, local routine data suggested a problem that needed to be assessed, while the second most common reason was response to a national policy initiative (28\%). 
Table 21: Selection of topics for analysis (1997-2000)

\begin{tabular}{|l|c|c|c|}
\hline $\begin{array}{l}\text { How topics got on to the local or national } \\
\text { agenda }\end{array}$ & $\begin{array}{c}\text { Number of } \\
\text { studies } \\
\text { (n=25) }\end{array}$ & $\begin{array}{c}\text { Percentage } \\
\text { of studies }\end{array}$ & $\begin{array}{c}\text { Percentage } \\
\text { of studies } \\
\text { LHA }\end{array}$ \\
\hline Local routine data suggested a problem & 10 & $40 \%$ & $24 \%$ \\
\hline Already chosen as area for change/ review & 2 & $8 \%$ & $19 \%$ \\
\hline Anecdotal evidence of a problem or neglect & 2 & $8 \%$ & $18 \%$ \\
\hline In response to a national policy initiative & 7 & $28 \%$ & $13 \%$ \\
\hline Local pressure external to HA or MOH & 0 & $0 \%$ & $5 \%$ \\
\hline Pressure from an individual within the HA or MOH & 4 & $16 \%$ & $7 \%$ \\
\hline In response to organisational change & 0 & $0 \%$ & $5 \%$ \\
\hline Regional priority or initiative & 0 & $0 \%$ & $2 \%$ \\
\hline Opportunistically undertaken as funds available & 0 & $0 \%$ & $1 \%$ \\
\hline Don't know & 0 & $0 \%$ & $8 \%$ \\
\hline
\end{tabular}

HA, Health Authority; MOH, Ministry of Health; LHA, London health authorities. Note: The categories used are those of Hensher and Fulop (1999), for comparison purposes. (See Table 15, p.153, for a description of the various categories).

Pressure from an individual within the organisation led to $16 \%$ of HNAs, but organisational change, such as redefined boundaries or new organisations, did not lead to any new needs analysis.

\section{HNAs and decision-making}

The relationship between HNAs and decision-making during the period under study, 1997-2000 (Health Funding Authority), was of particular interest in this research. It was hoped to obtain detailed information regarding qualitative and quantitative changes to services, but interviewees were not able to respond with sufficient detail. However, it was possible to obtain clear responses regarding whether any action (change in services) had been taken, and whether a service review had been conducted (without change) in response to the assessment. Actions could include changes to existing services, or the funding of new services. Reviews included service reviews without changes to services, or led to a further round of analysis or research. No action meant that nothing was done at all. This information was cross-tabulated with data regarding the method by which a topic had been selected for detailed needs assessment; the result is shown in Table 22 below. 
Table 22: Relationship between method by which a topic was selected for detailed HNA and subsequent impact of needs assessment on decision-making (1997-2000), compared to LHA data.

\begin{tabular}{|c|c|c|c|c|c|c|c|}
\hline \multirow{2}{*}{$\begin{array}{l}\text { Reason topics were selected and } \\
\text { perceived actions resulting }\end{array}$} & \multicolumn{2}{|c|}{$\begin{array}{c}\text { Action } \\
(\%)\end{array}$} & \multicolumn{2}{|c|}{$\begin{array}{l}\text { Review } \\
(\%)\end{array}$} & \multicolumn{2}{|c|}{$\begin{array}{l}\text { No action } \\
\quad(\%)\end{array}$} & \multirow{2}{*}{$\begin{array}{c}\text { Number of } \\
\text { studies } \\
(\mathbf{n}=25) \\
\text { NZ }\end{array}$} \\
\hline & NZ & LHA & NZ & LHA & NZ & LHA & \\
\hline Local routine data suggested a problem & 70 & 79 & 10 & 10 & 20 & 10 & 10 \\
\hline Already chosen as area for change/ review & 100 & 77 & 0 & 18 & 0 & 5 & 2 \\
\hline Anecdotal evidence of a problem or neglect & 0 & 65 & 100 & 35 & 0 & 0 & 2 \\
\hline In response to a national policy initiative & 28 & 50 & 44 & 50 & 28 & 0 & 7 \\
\hline In response to organisational change & 0 & 50 & 0 & 0 & 0 & 50 & 0 \\
\hline Local pressure external to $\mathrm{HA}$ or $\mathrm{MOH}$ & 0 & 44 & 0 & 44 & 0 & 11 & 0 \\
\hline $\begin{array}{l}\text { Pressure from an individual within the HA } \\
\text { or } \mathrm{MOH}\end{array}$ & 0 & 33 & 66 & 17 & 34 & 50 & 4 \\
\hline Numbers of NZ reports (and \%) & \multicolumn{2}{|c|}{$11(44 \%)$} & \multicolumn{2}{|c|}{$9(36 \%)$} & \multicolumn{2}{|c|}{$5(20 \%)$} & 25 \\
\hline
\end{tabular}

HA, Health Authority; MOH, Ministry of Health; LHA, London health authority. Note: The categories used are those of Hensher and Fulop (1999), for comparison purposes. Action means service change; Review means a service review but no change resulting to services; No action means that nothing was done at all.

In New Zealand, only $44 \%$ of needs assessments resulted in any action. This suggests a heavy preponderance of relative inaction following HNAs, in contrast to the NHS where $66 \%$ of needs assessments were perceived as leading directly to concrete changes in service provision (Hensher and Fulop, 1999). In New Zealand, a further 36\% resulted in a service review but no change. Such a review could represent a good result for a service in that the service may not have required change; or it could reflect the ineffectiveness of the HNA in bringing about change. The data show that no action was taken as a result of up to $20 \%$ of HNAs.

Even allowing for the smaller numbers in this New Zealand study - 25 compared with the 109 similar HNAs included in the UK study sample - it appears that the impact of HNAs in the 1997-2000 period in New Zealand was much less, by even as much as a third (44\% versus $66 \%$ ). The implications for HNA in New Zealand will be discussed later.

As in the UK study, a needs assessment was most likely to lead to policy action when the priority emerged from the analysis of local data or when detailed needs assessment focused on issues of specific local relevance. HNAs initiated in response to a national 
policy initiative were much more likely to result in a service review, or no action. None of the HNAs initiated by an individual within a health authority or the Ministry of Health resulted in any action; this was judged by staff to be either a result of failing to gain sufficient support for the initiative in the first instance, or because they were for research purposes only.

Further descriptive information was gained from the semi-structured interviews with managers and researchers regarding their experiences in conducting HNAs. These results are now described under four main headings: planning and conduct of HNAs; prioritisation; purchasing decisions; and consultation.

\subsubsection{Planning and conduct of HNAs}

The effectiveness of the planning and conduct of HNAs was dependent on clarity of objectives, who initiated the HNA, resourcing, stakeholder involvement, community involvement, methods, and the availability and quality of data.

\section{Clarity of objectives}

The need for "a very clear idea about what questions people wanted answered" was a common response regarding objectives, or lack of them. One participant commented, "An exercise like this requires a very good analysis of the problem before you start getting into need." Yet no significant discussion of the meaning of health need was found in any of the documents during the research.

In some cases there appeared to be a genuine desire to "plug the information gaps" or to conduct "some systematic evaluation and planning." One researcher sought to "get some baseline data so that we could track whether or not the funding decisions that were being made were having an impact on population health status over time." (in other words, health status monitoring). 
Some HNAs had multiple goals:

I wanted to get some key directions that would be publicly available, I wanted people to be better informed about current services and current health issues and I wanted to get some sort of consensus from the major stakeholders about the direction. (manager)

This observation came from a manager who was seeking to prepare a strategic planning document. The need for clarity regarding the objectives of HNAs was mentioned by a number of interviewees who frequently noted that objectives can be confused, especially if there was "strong political interest and public conflict" regarding an issue. A HNA may not be the right approach for solving a purchasing problem:

A HNA might not be the best process or approach in every situation, so in some situations no matter how good one's HNA, it is not going to influence the shape of things for other reasons and so it shouldn't automatically be done. (manager)

\section{Who initiated the HNA?}

Reasons for commencing HNAs included the special interests of individuals, local concerns, regional initiatives and national initiatives. Statutory requirement was uncommon, being present in only one case. Interest from an individual resulted in a HNA in at least five cases. Reasons given included a need for utilisation data (unavailable from any other source); a need for data to form an evidence base for contracting; "needing a product to focus on" (a newly appointed manager); and deep concerns regarding a current lack of specific treatment services (for example, Māori mental health services). Local concerns were also common and examples included disquiet regarding availability of services in a local council area; concern regarding changes to maternity care and the impending closure and replacement of the local maternity unit; and "particular responsibility on the part of the purchaser to identify the needs of the people of ... to purchase services to meet their needs."

Regional concerns related to a high incidence of inappropriate admission to hospital for preventable illness amongst Pacific people; consulting on the siting of the Wellington City tertiary hospital at election time; a lack of regional primary care data; the need for data prior to entering a contracting round; the "obligation of the purchaser to understand 
the health needs of its population as a fundamental driver of the purchasing cycle"; plugging some gaps in the information base; and planning purposes. National initiatives related to measurement of health risk factors; health status and health service utilisation; comparing data with previous national surveys; forming a national resource and initiative to inform the health sector and public about the health of New Zealanders; concern as to the quality, co-ordination and funding of services; and lack of progress towards integration of particular national health services.

HNAs conducted by the Ministry of Health constituted a special case. In a number of instances they were used to develop a health strategy document. For example, the Child Health Strategy commenced with some work in needs assessment.

\section{Leadership}

A significant issue was the need for clear leadership regarding HNA. Several researchers commented on this requirement, suggesting that both leadership and organisational commitment were important. Linked to this was a need for clear governance structures for projects, suggesting that where this had been present a project was more likely to be successful. This appeared to be particularly important for health authority projects, which were more likely to involve the community and health service providers than were Ministry projects.

Several managers and researchers mentioned the importance of a committed team that maintained continuity. Changes of personnel during projects led not only to loss of knowledge and data, but also to disruption of the project. Organisational commitment was seen as vital if the project was to succeed.

\section{Skills}

A number of interviewees commented on the importance of drawing together the right team with the appropriate technical skills to undertake the task. Mostly this required researchers with quantitative analysis skills, and experience in public health. One noted 
regarding the need for specific cultural skills, "In retrospect the team wasn't broad enough ...there was no Māori or Pacific people on the team." Public Health Medicine Registrars were often involved in projects as part of their training, sometimes leading projects without much supervision. This led one to comment, "That was partly kind of being dropped in the deep end."

Several researchers commented about the lack of training resources and information for undertaking HNAs. They felt that it would have been useful if the Ministry had given some guidance to the sector regarding how to conduct a HNA, manage epidemiological information, translate it into assessment of future health needs, and determine appropriate services, locations and facilities. Some surprise was expressed when it was explained that this resource was made available in 2000. Another pointed to the need to share learning and knowledge in order to pull "quite an important document" together.

\section{Resourcing}

Most health authorities, in planning HNAs, appeared to have underestimated the requirements of funding, time and workforce. Comments such as "a small underfunded project on which I spent a great deal of time," "we should have had more time," "very labour-intensive, high resources," "got so few people around who can actually understand what a needs assessment is," "we also don't have the people with the skills to actually undertake the HNAs," and "time, capacity, resources" were common from the HNAs. The effects of constant restructuring in the health sector were considerable:

Because of the reorganising, restructuring that happened in the Ministry, sadly instead of a group of ten people who could contribute to this, there was basically just me, with a little bit of help, and a tiny bit of money to encourage getting some help from a few friends, most of whom worked virtually for nothing on this. (Ministry of Health researcher)

Many of the HNA processes were characterised by changing staff, some leaving early and others coming in part-way through the process, unclear about the history or genesis of the project or even some of its objectives. Those who had left early were often unaware of the outcome of the project, and in several instances had not seen the final report. One manager commented, "What happens when someone leaves is that there is a 
big gap, and then someone else comes along and takes it, and they have no buy-in to a previous document and think that they need to start again." No doubt this led to considerable duplication and loss of direction. The impression gained is one of relative inexperience in the sector, many people on a steep learning curve, many lessons learned on the way, and struggles in the face of under-resourcing.

The other significant issue related to continuity is that over $85 \%(29 / 34)$ of those who were interviewed were now either in different positions within the health service, or had moved out of the health sector altogether. Less than five percent overall were still working in the general area of the HNAs that they were involved in initially. It seems that the opportunity cost of health reforms leading to changes in health employment must be significant, with potential duplication of previous work, loss of data, difficulty in retrieving data from datasets, and loss of 'soft' knowledge within the sector.

A public health physician acknowledging the need for adequate resourcing said that under-resourced assessment "risks being superficial." Others spoke of very small budgets "running away" with them in the middle of the project, leading to compromise of the project at the end. Assessors' comments led to the conclusion that many of them were short of time, funding, and skills to complete the task. A number felt that they were pressured during the HNA and that the quality of the work was compromised. If this was the case, it raises a question regarding the reliability of some HNAs reports.

Contractors were used to a significant extent during HNAs. Their roles ranged from full conduct and reporting on findings, to sub-contracting for survey work and focus groups. AGB McNair ${ }^{31}$ were used for a number of surveys in Auckland, while the Ministry of Health contracted Statistics New Zealand ${ }^{32}$ to conduct the Ministry surveys. The use of contractors for HNAs was not always considered satisfactory. There were concerns

${ }^{31}$ AGB McNair is a private research company that conducts research for a wide range of clients utilising mainly surveys, focus groups, and opinion polls.

${ }^{32}$ Statistics New Zealand is the official New Zealand government body responsible for collecting national statistics, including the national Census. 
regarding costs, quality of work and differing perspectives on how the research should be conducted.

\section{Stakeholder involvement}

The process of strategy development is probably ultimately as nearly as important as the outcome because the reason you embark on the strategy normally is to get some kind of change in the world. Developing a strategy that is going to be effective, the process you go through for development, the way you engage stakeholders actually determines what you are going to be able to do with the final product. (manager)

Stakeholders in a number of HNA projects were members of steering committees. Project managers and researchers recorded their opinions on the usefulness of such committees. Statements such as, "those people should be involved right from the outset" are indicative of the perceived value of including stakeholders. Regarding funders, interviewees expressed the view that the success of the project, particularly regarding implementation of the recommendations, was related to involvement of the funder of health services from the start of the project. One funder similarly commented that it was important to "be able to sit alongside the HNA people and to be working on it together." The same funder also said that the funder should not "influence the HNA to the extent that it becomes totally biased in order to support the funder's particular framework."

There were some interesting examples of stakeholder involvement that had worked well for intersectoral projects. This represents a relatively new finding, as traditionally the health sector has been somewhat isolated from other related sectors in New Zealand. In a project commissioned for a community health partnership, a steering committee with representation from the Ministry of Health, Local Council, and community, including Māori and Pacific, appeared to have worked well, according to various representatives. Another project manager spoke of the benefit of a multi-stakeholder steering group "to generate some sort of cross-provider, cross-sectoral enthusiasm for health improvement strategies." Yet another spoke of the benefit of involving purchasers, the Ministry of Health, Chamber of Commerce, and Pacific people in a project governance group in 
order to "ensure that the process was totally transparent and inclusive of all stakeholders."

Many assessors found the process of managing stakeholders quite difficult. Tensions arose between the advocacy positions of providers and communities, and the views of those within the Ministry. One researcher commented, "You can imagine how difficult it was with different people having ownership and trying to bring it together coherently and then coming up against a Ministry filter which raised a different kind of concern about advocacy and about accuracy of data sources and consistency."

The degree to which the community was involved in HNAs varied considerably according to the nature of the assessment. Community involvement was not required for large-scale epidemiological exercises such as Our Health, Our Future, which analysed and presented mostly mortality and morbidity data.

Other kinds of HNA exercises required community input. But working with communities was regarded as challenging, and required significant investment:

Well, first of all, I think that research like this if it is really going to be useful has to be done with communities and not on communities - it has to be engaging. Secondly, I think people have to be prepared to go out and identify and work with communities and be there for communities if they are really going to do this work effectively; and thirdly, there has to be a significant investment made in the infrastructure in communities for them to be able to do these sorts of things. (academic researcher)

Building trust through a long-term sustained relationship was reported to facilitate working with communities. "I really think I have developed a real sort of trust and identity in terms of actually working with the community and trying to bring some of these things about," reported one researcher. Another commented on

the number of very fine pieces of epidemiological public health work, but there were two factors missing from most of them, one of these being community engagement and community data, and the other being involvement of the purchaser from the outset so that the purchaser could build a relationship with the community and understand the meaning of the data. (academic researcher) 
This is supported by another researcher, who commented, "By going out into that community, meeting with the people and talking in detail about the health issues for them, I mean we actually have some information now...something we haven't had in the past." The benefit was seen as going beyond merely obtaining data, and gathering support for a document, especially where it was leading to a strategic plan. In discussing the importance of the community approach, including consultation with community groups and health professionals, one manager stated: "What we were trying to ensure was that there was active participation, involvement and ownership of the document at the level where it was going to make the greatest difference." Several researchers commented on the value of allowing communities access to their own data. Referring to separating Porirua and Kapiti data in the Porirua Kapiti Healthlinks project, one said, "Getting decent data for each of them was really powerful for each of those communities." Those communities acted upon a significant number of recommendations from the HNA, and it was predicted that the communities would be in a much better position in the future to work with DHBs "to get things that work well in their communities."

It should be pointed out that the research findings reported above refer to obtaining community perceptions of needs during the HNA process, as distinct from community consultation on health authority plans and directions, which is quite a separate process.

\section{The role of $\mathrm{HNA}$ in community development}

Further along the scale of community engagement lies the community development model, the use of which has been uncommon in New Zealand. One researcher observed that "People have to be prepared to go out and work with communities and identify with communities if they are going to do this work effectively." For needs assessment to be of value, he noted, "finding the right communities of action is important so that when we are doing HNAs we can provide information which will activate those communities or give them something to work with." This process "helps communities to engage....and that helps build up their social capital and cohesiveness as communities, which is health-promoting quite apart from what the input might be with respect to service development." 
One example of the use of HNA in a community development model was a mental HNA in a Māori community on the East Coast, which resulted in purchasing "...true intersectoral stuff really, rather than just health service provision. It was a totally new concept where there is now huge community engagement, huge community involvement and community ownership... that community said within a few months they could already see outcomes that they were looking for with their kids." They concluded that we should "...give communities a much greater share of ownership and control, and their own really self-determination about things that they know will work for them."

Yet another researcher considered that the community development approach was useful for "closing the gaps" because it empowered communities. Another manager talked of "having the courage...to give communities a much greater share of ownership and control and their own self-determination about things that will work well for them". Others spoke of the value of community action through partnerships with local and central government.

In Porirua and Kapiti, a community development approach had been used, with a high level of engagement with the community. This was characterised, according to a researcher working in that community, by:

... a long term engagement process, ... not a one-off consultation process. That there is a period of sharing information that needs to go on and sharing it both ways, the challenge often is how to value... people's stories as well as valuing quantitative information and to use the two to inform each other and to challenge each other because it needs to go both ways. I think the value of providers and communities coming up with the things that they think work well, because they are not always what that data shows at all...(researcher)

Community development required a substantial commitment of time and resources and again, as the same researcher commented:

To do the sort of thing that we did in Porirua and Kapiti is quite intensive... and I would have expected that that is something that you would do over a year or so in communities with big outcome disparities rather than something that you would attempt to do across the whole of your District Health Board. (researcher) 
The role of the community as a community development partner was strongly supported by a statement from one experienced researcher:

If you can actually go out and take your researching capacity and sit down with communities and listen to them, there are some wonderfully imaginative, resourceful and capable people out there who in their own way often manage extremely difficult lives with very modest resources to draw on and could be wonderful partners in the health system if we ever bothered to try to realistically engage them in the doing of some of these things. (academic researcher)

Although the use of the community development model was less frequent in New Zealand, the researcher quoted felt that where it had been used, that HNA appeared to have had an impact on forming health service plans.

\section{Methods for HNA}

Researchers conducting HNAs used a wide range of research methods. They ranged through surveys, focus groups, key informant interviews, epidemiological and statistical approaches, to community group meetings, consultations and hui. ${ }^{33}$ Morbidity and mortality data were common starting points. But the views of several researchers regarding the use of such data were encapsulated by one researcher, who said, "It was limited because it was mainly statistical analysis and it would have been better to have done a more comprehensive combination of methods, both quantitative and qualitative."

The lack of methodological models for HNA was considered a problem prior to publication of Health Needs Assessment for New Zealand: Background paper and literature review, and the companion Ministry of Health guideline. (Coster, 2000, Ministry of Health, 2000b)

Researchers commented on the desirability of carefully prepared questionnaires to ensure that data were collected in a form that was usable for analysis. The use of standardised data definitions was also considered to be important.

\footnotetext{
${ }^{33}$ A hui is a consultation with Māori groups, which are often based on Iwi (Māori tribal) groupings.
} 


\section{Data availability, quality and analysis}

Many issues were raised regarding data including availability, quality and analysis. One feature that attracted comment from at least three researchers was the difficulty in obtaining data from the primary care sector. One said, "As usual, no primary health care statistics were easily accessible, and it is a problem that primary health care always gets missed out of needs assessment because of the lack of availability of data." Another said, "Primary health care data is (sic) always difficult or impossible to get... and HNA would be much better rounded if you could get that, and if it were more accessible." Researchers and managers commented that the main issue was the reluctance of primary care organisations (for example, privately owned Independent Practitioner Associations) to allow access to their databases by publicly owned DHBs, which in turn were purchasing services from them. Those in primary care organisations felt that their stake in the sector could be adversely affected by sharing information with the public purchaser. But the purchasers viewed it differently and considered that it was in the public interest for the information to be made available.

Difficulties in accessing data however were not confined to primary care. As one researcher put it:

Well, one of the problems I have with the data is getting access to it (sic) after successive rounds of restructuring and successive rounds of passing on the information from one organisation to the other. Although we now know where the data is (sic)... it is very difficult to get access to the information because there are all sorts of questions about who owns the data and how much access you should have to it (sic). (researcher)

This comment referred particularly to data held initially by an RHA, then the Health Funding Authority, and finally transferred to the Ministry of Health. Another expressed concern related to protective ownership of data, particularly those collected by the Ministry of Health. It was considered that the Ministry should "take a public-good view in relation to the wealth of data that sits in their databases." It was felt important that skilled researchers should have access to these data for research purposes. It was difficult to validate these claims regarding access to Ministry-held data, particularly when specific instances were not made available for checking. The Ministry of Health policy is that data will generally be made available for researchers, although only in 
aggregated form. This may have presented problems for some managers and researchers seeking data with patient identifiers in order to track utilisation of health services, and expenditure, by chronic disease categories.

Lack of health outcome data was a general concern, for example regarding maternity and neonatal health outcomes:

Several of the recommendations in the review related to improving the collection of outcome data in maternity care services, and I think that some of the steps are only just being taken with the reestablishment of maternity and infant mortality committees and the collection of data. It was difficult at the time to make too many judgements about the outcomes of maternity care, other than consumer satisfaction and very broad population indicators... (manager)

However, despite concerns regarding the availability of data, the Ministry of Health 1996/97 New Zealand Health Survey and 96/97 Disability Surveys were both held in high regard as useful sources of information on the health of New Zealanders. It was considered important for health planning purposes that the surveys should be repeated every five years, as planned. It was suggested that linking these surveys to the Census would make the datasets even more valuable, in that the population denominator would be clearly established. The importance of up-to-date accurate datasets that were reliable and valid for the work being conducted was stressed, as was the value of a time series. One respondent observed regarding the value of involving Statistics New Zealand:

Certainly it was extremely valuable to have Statistics New Zealand working with us as an expert data collection machine. They are well oiled, they know what to do and they are thoroughly professional in terms of their sampling, design and so forth. (manager)

Several researchers raised questions about data quality such as 'small number' problems in New Zealand, and the accuracy of data" such as immunisation figures. The collection of ethnicity-linked data was considered imperative, as many data sets did not record patient ethnicity, and this was considered important in order to identify Māori health need. 
The validity of datasets was also considered an issue. The National Health Surveys conducted by the Ministry of Health had sampled 7,500 people nationally. ${ }^{34}$ Ethnicitylinked data analysis was considered unreliable for denominator populations much smaller than the populations of the Regional Health Authority areas (approximately 900,000 people). The Northern RHA, in considering their requirements for the North Health Survey, asked the Ministry to over-sample in the region, to improve the reliability of the survey data.

\subsubsection{Prioritisation}

Health needs assessors noted that a significant number of reports contained no recommendations (document analysis confirmed that recommendations were absent from $42 \%$ of reports). Views regarding the usefulness of recommendations varied, from emphasising the importance of recommendations, through to scepticism. One researcher reported, "We weren't actually convinced that if we made recommendations that people would have acted on them anyway," and others took the somewhat naive view that the data would "stand on its (sic) own and therefore speak for itself". It was disappointing to find so many HNAs without recommendations, prioritisation, or even summaries.

In the course of interviewing, it became apparent that the Porirua and Kapiti projects had prioritised recommendations at two levels, based on expert opinion, with implementation fully dependent on new funding. The prioritisation framework of the Health Funding Authority was not utilised, or at least not overtly. As noted earlier, as a consequence of the needs assessment demonstrating the requirement for better access to health services, the Minister of Health agreed to establish a Health Impact Zone in Porirua. This was considered to be a positive outcome from the Porirua Kapiti Healthlinks project.

Most managers agreed that prioritisation should become standard. Where some attempt had been made at recommendations for prioritising of funding, interviewees were asked

\footnotetext{
${ }^{34}$ The population of New Zealand reached 4 million people in 2003.
} 
whether government priorities had influenced or even pre-determined those recommendations. It should be remembered that the New Zealand Health Strategy and related strategies had not been published at this stage. One or two researchers commented that they felt that recommendations were generally consistent with government priorities, as expressed through public health goals and other Ministry documents. In children's health, for example, priority populations were identified Māori and Pacific children, children with chronic disabilities, and children living in poverty. The Māori Health Team of the Health Funding Authority had developed Eight Māori Health Gain Areas, ${ }^{35}$ and these were consistently used in the prioritisation framework for Māori health service purchasing by the Health Funding Authority. Generally, in making recommendations for service development, managers and researchers were cognisant of government priorities for health services.

\subsubsection{Purchasing decisions}

The place of HNAs in influencing purchasing was contextualised by a manager:

HNAs cannot be done in isolation from the reality of purchasing decisions, and the various factors that impact on purchasing decisions; political factors, ethical and social obligations, shape of existing services, competing interests and all those sort of things. (manager)

It was acknowledged that HNAs had not previously fitted neatly into the decisionmaking cycle of funders and providers and that decisions had often been made on the basis of cost or even political expediency, rather than need. Additionally, it was noted that there were powerful clinical, political and Government bodies that have had a stake. One respondent commented, "There is a political arithmetic that operated alongside needs assessment arithmetic, and I am not sure that you can ever remove that political dimension from it."

\footnotetext{
${ }^{35}$ The Māori Health Gain Areas are: immunisation; hearing; smoking cessation; diabetes; asthma; mental health; oral health; and injury prevention. Minister of Health (2000d) New Zealand Health Strategy. Wellington: Ministry of Health.
} 
A blurred picture emerged of the impact of HNA on health services purchasing. One public health physician reported, "I am not sure that I could point to any particular policy or new purchasing that happened directly as a result." Another reported, "I think that it influenced the purchasing direction substantially." HNAs were also used to a significant extent to justify decisions already made. One manager reported:

I know that the HNA has been used quite substantially within the RHA itself by those who are on the contracting front, and presented to their counterparts in the other RHAs as the evidence for some of the decision-making process, including for decisions made internally beforehand. (manager)

Another said, "There were a number of cases where the work that I was doing has been used for justification." Commented one health needs assessor, "When you are looking at health services planning you should do the HNA at the beginning, rather than at the end when the decision is being made."

Service-level purchasing was a possible action in response to HNAs, particularly where recommendations for action were made - for example, in Porirua and Kapiti new services were implemented to improve changes to public transport to facilitate access to specialist services. In one interesting example of the use of an HNA, a public health physician manager said that a report was tabled in Parliament, causing the Leader of the Opposition to ask why the Government was not doing anything about these "terrible inequalities." Political action following the Child Health Strategy resulted in the then Minister of Health finding an additional \$13 million to go to Well Child health services. One manager commented that "This was a really significant, a dramatic, injection of funding into well child health, which had been unprecedented prior to that, and a significant amount of that of course went into the priority populations."

Following a HNA of rest-home bed requirements for health care for the elderly in the Midland region, there was a change of purchasing direction, with disinvestment in rest home beds, but new investment in dementia beds. This was the only needs assessment in the study where there was evidence of subsequent disinvestment. 
Following a Pacific needs assessment, new funding was committed to the strategic directions that flowed from that assessment, and a new Pacific Health Unit was established in the Ministry of Health. Funding remained to purchase existing services as a result of need demonstrated by the West Coast needs assessment when up to $\$ 3$ million was under threat of withdrawal by the Health Funding Authority.

On the positive side, some health managers felt that their HNAs were of lasting benefit, "probably influencing some purchasing... and that the DHB was using it as a reference source for their needs analysis and strategic planning." Another lasting benefit was summarised thus: "the whole intent was to repeat these processes periodically so that you could...also monitor the impact of what you were doing."

Strategically, HNAs have brought about a "much more co-ordinated approach to health issues," according to one City Councillor. In this case, intersectoral approaches by local government and the health sector have resulted in communities and the Council working together on issues related to policy and service.

Whilst some researchers and managers were unclear regarding the benefit of studies that they had conducted, others felt that there were clear outcomes leading to a different approach to purchasing. One respondent felt that the data collected were invaluable for the development of the New Zealand Health Strategy, and had also informed other strategies. In Porirua, it was felt that one key outcome of the HNA was a new relationship between the community, local government, and central government, which allowed them to work on issues together.

Needs assessments should be used to inform decision-making, or highlight areas for decision-making, and to guide policy and the purchasing of services. Furthermore, the real value of needs assessments lies in subsequent evaluation of health need to ascertain whether health service purchasing has been beneficial to improve health outcomes. (researcher) 
This reflected an interesting view that HNAs have a role not only in the initial assessment of need for services, but for ongoing evaluation of the effectiveness of health service interventions.

With regard to health outcomes, most people questioned on this subject understandably felt that it would not be possible to ascertain whether there was an improvement in outcomes as a result of the HNA. Several managers felt that there was clear evidence of improvement in health outcomes, for example in health care for the elderly, child health outcomes, and Pacific and Māori health outcomes (particularly resulting from improved access to care). Others could record no improvement in health outcome.

Data from Ministry of Health-led HNAs were frequently used to inform and develop health strategies. Such examples included Making a Pacific Difference; Our Children's Health; Review of Maternity Services in New Zealand, and Kia Tu Kia Puawai. Others, for example Taking the Pulse and Our Health, Our Future, were used more widely. Some were used to monitor public health outcome targets. One senior Ministry of Health manager said that his job description required him to implement a strategy that was based on a major HNA, noting that he could name six initiatives that were a direct result of the HNA.

Interviewees identified a number of barriers to implementing findings and recommendations arising from HNAs. These included organisational change during the needs assessment, such as restructuring of the purchaser; lack of 'ownership' of the report (usually due to lack of purchaser involvement at the outset); organisational and workforce capacity; funding constraints or competing priorities; the need for intersectoral action; lack of political commitment; and political action. Where there were competing priorities it is understandable that some or all of the findings and recommendations of a HNA were not implemented. One researcher reported that she had found that one way to improve the chance of recommendations being taken notice of was to "sit down with a decision-making person and present it to them and lead them 
to the recommendations, because they could then see the whole process of research and implementation."

The importance of establishing a firm link between HNA and purchasing was emphasised, with reference to a mental health HNA:

Here was an excellent piece of work but unfortunately it never went anywhere. I think it is a good example that unless there is real commitment at a management and purchasing level to actually make things happen, good work like this can be done and it is just left as an interesting piece of work on a shelf which has never ever changed purchasing patterns. (researcher)

Such comments as "the ability to implement anything becomes quite difficult," and "they make the recommendations but there is no infrastructure to follow it up" were quite typical. One respondent reported, "I don't think that at that time there was any particular concept about the need for an implementation plan." Another said of the Pacific Plan, "It's impact is more in an inspirational sense, you know that these are good ideas and these are the kind of things that we should be doing, but I think that you have to remember that it was ground breaking, and it was the first." And later, "It was driven by a lofty vision, but not perhaps informed by implementation."

In one notable example, managers in the funding organisation vented extreme frustration at the actions of a Government department that blocked the launch of an initiative to deliver services in the community "...right up to the night before the ... were trying to stop the launch of the document" and "just about killed it." In another case, a needs assessment document ready for final public consultation was blocked from publication because final approval could not be obtained, reportedly because of the political sensitivity of the questions regarding new hospital facilities in Wellington, and possibly for reasons linked to the continuing role of the Health Funding Authority.

One researcher commented on the events surrounding the question of expansion of secondary care services at Porirua:

And then it all came to a bit of a head as it has done from time to time, and has done again over the future of the Kenepuru hospital, and people got all excited 
about that at that time, and there were the usual sort of political shenanigans and undertakings given as to how services were going to improve in Porirua and all that sort of stuff but the reality was of course when push came to shove and resources got constrained and the pressures in relation to the medical school and established medical interests and then Wellington Hospital came to bear on the process and in way or another the services in Porirua.... (academic researcher)

The role of the purchaser was considered to be of central importance in implementation, but in several instances it was questioned whether the purchaser even agreed with the recommendations of the needs assessment. One researcher commented that "The detail of the needs analysis and the report was first class, it was just at the end hour there was lack of commitment on the part of the purchaser to honour and endorse the recommendations in the report, and that's where it fell down." The role of the purchaser as a stakeholder in HNAs was stressed by several people, who suggested that the purchaser should be a member of steering committees of projects from the outset.

Implementing new services in response to recommendations from a needs assessment can require, "quite a lot of capacity building that needs to happen. I think if you look at workforce issues and where the gaps are too, I think that is probably quite a biggie really." Others voiced the need for additional financial resources in order to implement findings from HNAs.

\subsubsection{Consultation}

\section{Community}

More than half the HNAs studied involved consultation with communities, and sometimes with providers, especially where HNAs led to policy or strategy documents. Community consultation was not always done well, leading to the criticism:

Classically what happens is that an outside group, or the Health Funding Authority, comes parachuting in... and they do a needs assessment in a relatively short period of time, with all the resources available to them, and then they send a glossy report back to the community and say you people have got a month to provide some submissions on this and then we will decide what to do. I mean it is a totally, a totally inadequate way of actually working with communities and the results of that sort of thing generally are pretty sparse and not very informative. 
(This) leads to people who did it of course to say, 'well there we are, the community is an ignorant bunch, they have got no idea about these things, I don't know why we bother to ask them at all'. (researcher)

In response to concerns regarding the adequacy of consultation processes, the Health Funding Authority developed guidelines for consultation with communities (Health Funding Authority, 2000b). There is no clear evidence in the few HNA documents that were published after the publication of those guidelines that they were used. They were beginning to be used by the Health Funding Authority for community consultation regarding proposed service changes in the last year of the HFA's existence.

Some of the consultations proved to be very difficult. The National Health Committee decided that it would be useful to hold a maternity forum to obtain consumer and stakeholder opinion regarding the Review of maternity services in New Zealand, one of the HNAs in this study. The process was described as "tricky and difficult... and I don't think it entirely met the objectives of what we would have thought when we started planning." Yet it was helpful in that it "determined the somewhat varying ... and entrenched views." The role of consumers in the debate was considered to be very helpful.

Some managers reported bad experiences with community consultation, mostly as a result of community reaction following poor consultation processes in the past:

You know the funders have traditionally had bad press with their engagement in the way they have gone about it in the past. A lot of people who are working for the $D H B$ s now are very, very risk averse when it comes to engaging with the community. I understand that, but at the same time there are a lot of people who do want to engage directly with the community, but they want to have the time to plan it and do it properly and make it meaningful, and that just takes time. (manager)

In at least one case the HNA team ignored the results of consultation, prioritising children's health ahead of the health care needs of the elderly. "We came back from these consultations and decided to disagree with the community and prioritise child health consistently." This example occurred in a Pacific context, where there was a cultural preference for prioritisation of the health needs of the elderly. 


\section{Consultation with Māori}

Māori have a right to partnership and participation in health service planning processes, including consultation. Māori seek participation so that they can contribute their views and perspectives to the discussion, in addition to Māori health data collected through the HNA that is used to inform the debate. The Māori perspective is that "What Māori see very clearly is that participation in the decision-making process means that there is a combination of data plus experience and first hand knowledge." "Hopefully it will improve your ability to make the right kind of decisions," reported another manager.

Consultation with Māori means different things to different people. In the Ministry of Health, it often meant internal consultation with members of staff who were Māori to ensure the acceptability of a proposal to Māori people. In addition, staff of the Ministry were also required to work with Māori communities, to assess the usefulness and appropriateness of their findings for Māori. People talked about the difficulties of obtaining a Māori perspective, observing that there was no one view from Māori communities: "If I talked to more than one person I got three answers." This observation was not intended to be disrespectful, but simply an acknowledgement of the difficulties experienced in consultation with Māori. It signalled that local Māori views needed to be sought wherever possible, as there were no generalisations to be made; diversity of viewpoint was the norm for Māori. Similarly, it was felt that consultation with Pacific communities should be undertaken early and locally.

\subsection{Discussion}

This study had some limitations. It did not evaluate the quality of the HNAs from a technical point of view, or against any 'gold standard'. For each HNA there were only two interviews, generally with one manager and one researcher. The limited number of people interviewed in connection with each study meant that there was no opportunity for triangulation of information, but only for corroboration. Neither was there any opportunity to obtain an assessment of the effectiveness of the HNAs, other than from 
interviewees. Nor does the study evaluate the different contexts in which the HNAs were conducted, and the effects of those contexts on the HNAs.

The strength of this research is that it is the first research conducted on the effectiveness of HNA in New Zealand. The inclusion criteria for HNA reports are the same as those used for the London health authorities study, which allowed comparison between HNAs conducted in the UK and New Zealand. Although the number of HNA reports available for analysis (50) was less than expected, it was still possible to draw conclusions from them. Enough reports (25) were the subject of in-depth interviews to allow conclusions to be drawn regarding approaches to HNA during that period. The research identified a number of significant findings regarding HNA in New Zealand, including some related to Māori, and these are summarised below. These findings should assist DHBs with future HNAs. There was considerable interest in the research and a number of managers and researchers have requested copies of the final report.

The research evaluated HNAs conducted by the Ministry of Health, Public Health Commission, and health authorities including the RHAs and the Health Funding Authority, between 1991 and 2000. It evaluated the impact of HNAs on decisionmaking, service delivery, and health policy. It also considered the barriers to implementation of the findings from HNAs during this period. The research also provided valuable insights into the challenges faced by DHBs as they embarked on HNAs.

In summary, using the typology of the research, HNAs conducted between 1991 and 2000 were population-based (58\%), community-based (10\%), epidemiologically-based $(12 \%)$, comparative $(8 \%)$, corporate $(12 \%)$, and economic $(0 \%)$. The majority of HNAs were undertaken by the Regional Health Authorities (34\%) or the Ministry of Health (26\%). Decisions regarding purchasing health services were the key objective during the decade in $54 \%$ of HNAs, but despite this only $48 \%$ of reports made any recommendations at all, despite all HNAs having an objective(s). 
The more recent HNAs (1997-2000) fell into three broad groups: (a) HNAs that were predominantly epidemiological exercises, all using population-based approaches (12 reports); (b) Service-specific HNAs that used a combination of population-based and community-based approaches (8 reports); and (c) HNAs with both epidemiological and community data that used predominantly population-based approaches (5 reports). A significant number of reports, particularly those from group (a), were also used to inform new health policy, and health strategy documents.

The typology of reports in the sample of HNAs chosen for in-depth interviews (19972000) differed from those over the decade only in there being a greater proportion of population-based HNAs (72\% versus 58\%). During the years 1997-2000 decisions regarding purchasing of health services were the key objective of a similar proportion of HNAs (48\%). Fully one quarter of the reports were for research purposes. During this later period only $44 \%$ of HNAs led to an action regarding health service purchasing, $36 \%$ led to a service review with no change, and in $20 \%$ there was no action taken as a result of the study. A needs assessment was more likely to lead to policy action when the priority emerged from the analysis of local data or when detailed needs assessment focused on issues of local relevance (for example, service planning). These data were also similar to those of Hensher and Fulop (1999). They also found that needs assessment directly supported decision-making and action in two-thirds $(66 \%)$ of the studies examined, compared with New Zealand's 44\%.

Although there was little difference between the stated objectives of HNAs of the 19972000 group and those for the whole decade, there were some significant differences from those of the London health authorities. Those from London were more likely to be undertaken to resolve service-specific purchasing decisions (31.4\% versus $10 \%)$, and less likely to be undertaken for research purposes (14.1\% versus $26 \%$ ), or for other primary objective ( $2 \%$ versus $12 \%)$, or for multiple purposes $(9.4 \%$ versus $36 \%)$. These data suggest that HNAs in the UK were more focused in their objectives, which is not altogether surprising since their health authorities have been doing them for longer and are aware of the advantages of focused HNA for informing purchasing decisions. In this regard, just under two thirds of managers and researchers in New Zealand were 
conducting HNAs for the first time, which makes their efforts quite remarkable. HNAs in New Zealand were twice as likely to be conducted in response to a national health policy initiative.

Overall, the main observed function of HNA in New Zealand was identifying health problems, including health inequalities, and placing them on the policy agenda. This particularly included addressing the health inequalities of Māori and Pacific people. The second observed function was that of monitoring the health status of populations, which was done for a wide range of reasons, including the reallocation of resources. Other reasons included planning detailed changes once an issue had come on to the agenda; post hoc justification for decisions already made; and building ownership of decisions. These are similar to the findings of Fulop and Hensher who studied HNAs conducted by London Health Authorities between 1993 and 1997 (Fulop and Hensher, 1997, Hensher and Fulop, 1999).

It is interesting that HNA in New Zealand did not achieve anything like the impact of the London HNAs on service planning during comparable periods of study. The evidence suggests that HNA in the UK is more successful in linking with health service purchasing. The reasons for this are not altogether clear but there are at least two possible explanations. First, HNAs entered the health planning agenda in the UK a number of years earlier, and therefore managers were more experienced with needs assessment, and presumably with linking HNAs to health purchasing. Secondly, in New Zealand, managers and researchers have been more concerned with identifying populations and health profiles during successive rounds of health reforms than with needs assessment, even though the focus on HNAs for health service purchasing has been steadily growing. Locally, a much broader focus and purpose that are more descriptive and less decision-oriented have been taken. This may be related to the relative strengths of public health function in the two systems.

The present study found that clear objectives, decisive leadership, teamwork, communication, sound study design, adequate resourcing, skilled staff, sufficient time, 
community engagement and consultation (where appropriate), consultation with Māori, ownership by and involvement of stakeholders including funders and linkages with the implementation process were all important in ensuring that the HNA achieves impact on health service purchasing. Apart from the requirement to consult with Māori, these findings were similar to those regarding HNAs conducted by the London health authorities (Fulop and Hensher, 1997, Hensher and Fulop, 1999, London Health Economics Consortium, 1996).

Jordan et al. (2002) also described characteristics that defined an effective HNA in the NHS. They surveyed 62 HNAs conducted between 1993 and 1998. A number of themes emerged as important for the impact of HNAs on policy and planning. These included careful design, decisive leadership, good communication, involvement and ownership of the work by relevant stakeholders, support from senior decision-makers, appreciation of the political dynamics and engagement with local priorities, availability of resources, and an element of chance. In addition, they concluded that HNA did not occupy a central position in health service decision-making, remaining vulnerable to a range of factors over which those responsible for its conduct had little control.

During the period from 1991 to 2000, prioritisation gained increasing importance on the New Zealand health agenda through the work of the National Health Committee, and the Health Funding Authority. It is apparent from the present research that whilst theoretical frameworks for prioritisation were being developed, health authorities had not applied those frameworks and principles to prioritise health need. More commonly, health priorities were based on government public health priorities. The Māori Health operating team in the Health Funding Authority were actively using the eight Māori Health Gain Areas for purchasing priority health services. Linkages between HNAs and prioritisation were yet to be achieved to any significant degree during the 1991-2000 period.

Given that in order to assess health need an understanding of the meaning of need is desirable, it is surprising in the decade of HNAs under review, that no health needs 
assessors held a serious discussion regarding need. No ready explanation can be found for this; it would seem that needs assessors either agreed with the common definition of 'capacity to benefit' from health care services, or had simply not considered the matter. The debate about health inequalities was raised by some of the assessors, especially those from central government, but discussion regarding vertical and horizontal equity was not couched in terms of meeting health need, or requirement for services. It remains to be seen whether DHBs will discuss the meaning of health need.

The prerequisites for successful HNA in New Zealand have been established as a result of this research, and are remarkably similar to those found elsewhere, but with the additional requirements for partnership and participation of Māori people. The achievements of health needs assessors were significant, especially since they were mostly inexperienced with HNA. As a consequence, HNAs made less impact on health service purchasing than those conducted in the UK. This can in part be attributed to the topics selected for HNA in New Zealand. A number of lessons have been learned as a result of HNAs conducted by health authorities and these should assist DHBs with HNA and health service planning and purchasing in the future. 
Chapter 7: District Health Boards Assessing Health Need

7.1 Introduction

7.2 Transitional DHBs health needs assessment plans

7.3 Health needs assessment by DHBs

7.3.1 The Regional Health Needs Assessment Project (RHNAP)

7.3.2 Health needs assessments by other DHBs

7.4 DHB health needs assessment document analysis

7.5 Prioritisation

7.5.1 Context of prioritisation by DHBs

7.5.2 Prioritisation principles established by DHBs

7.6 Planning and Funding Manager interviews

7.6.1 Introduction

7.6.2 DHB health needs assessments

7.6.3 Prioritisation frameworks and prioritisation

7.6.4 Key influences on DSPs

7.6.5 Linkages between DAPs and budgets

7.6.6 Connections between health needs assessments and purchasing

7.7 Impact Factor - a measure of the impact of health needs assessments on planning and purchasing

7.8 Case Studies - DHBs

7.8.1 Case study A (Hutt Valley DHB)

7.8.2 Case study B (Hawke's Bay DHB)

7.8.3 Case study C (Whanganui DHB)

7.8.4 Case study D (West Coast DHB)

7.8.5 Case study E (South Canterbury DHB) 
7.9 Interviews with Ministry officials regarding expectations for health needs assessments and prioritisation

\subsection{Discussion}

\subsubsection{Introduction}

7.10.2 Types of DHB health needs assessments

7.10.3 Impact of DHB health needs assessments

7.10.4 Most effective and least effective health needs assessments 


\subsection{Introduction}

The aim of Part 3 of the research was to evaluate the HNAs and prioritisation undertaken by DHBs (see Chapter 5.3.3, p.138). The objectives were to:

(a) Evaluate the impact of DHBs' HNAs and prioritisation on health service planning and purchasing;

(b) consider five case study DHBs regarding the above; and

(c) consider the consequent relevance and effectiveness of government on HNA, prioritisation, planning and health service purchasing in the context of recent health reforms.

Government has been quite specific in stating its requirements for HNA and for the incorporation of priorities into District Plans, including them both explicitly in the NZPHD Act 2000. For these reasons it is useful to study HNA and prioritisation to determine their impact on decision-making and service delivery, and ultimately to evaluate the effectiveness of the policy.

\subsection{Transitional DHBs health needs assessment plans}

Transitional DHBs, formed in mid-2000 by appointing additional board members to existing Hospital and Health Services boards, were charged with preparing plans for new DHBs, to commence on 1 January 2001. Transitional DHBs were expected to submit their draft plans for the establishment of DHBs to the Ministry of Health, with final plans to be signed off by 1 December 2000. These plans were to be consistent with the New Zealand Health Strategy and guided by policy statements from the Ministry of Health. DHB establishment plans were to include plans for HNAs and prioritisation (Ministry of Health, 2000b). Specific Ministry requirements for HNA are shown below: 


\section{Box 4: Ministry requirements for HNA plans in transitional DHB plans}

- Health needs assessments

- DHBs are expected to analyse the needs of their communities, and consult with them on the ways to meet those needs, under the auspices of the New Zealand Health Strategy and New Zealand Disability Strategy. It is not expected that there would be separate methodologies for each DHB.

- Prioritisation

- DHBs are expected to have the capability to prioritise the needs of their communities, within the constraints of their service funding and the direction of the New Zealand Health Strategy and the New Zealand Disability Strategy.

Source: DHB Establishment Final Planning Guidelines (Ministry of Health, 2000a).

The key milestones set by the Ministry of Health for transitional DHBs are shown in the table below:

Table 23: Key milestones for DHB transition plans

\begin{tabular}{|l|l|}
\multicolumn{1}{|c|}{ Time } & \multicolumn{1}{c|}{ Milestone } \\
\hline 7 September 2000 & First draft of transition plan to the Minister \\
\hline 30 September 2000 & Feedback from the MOH on the first draft of plans \\
\hline 13 October 2000 & Second draft of the plan after review by the MOH \\
\hline 30 October 2000 & Comments to the Minister on the draft plans by the MOH \\
\hline 10 November 2000 & Recommendations to the Minister on plans \\
\hline 1 December 2000 & Transition plans approved \\
\hline
\end{tabular}

The Ministry of Health approved Transition Plans ahead of the establishment of DHBs by the New Zealand Public Health and Disability Act 2000, which commenced on 1 January 2001. The new DHBs were responsible for implementing plans for HNAs in accordance with Ministry guidelines. Advice to DHBs was provided in two publications (Coster, 2000, Ministry of Health, 2000b), along with indicators for HNAs and prioritisation noted in DHB Accountability Indicators 2001/02 (Ministry of Health, 2001b). DHBs conducted HNAs, which were then approved by Community and Public Health Advisory Committees of DHBs and subsequently ratified by incoming DHB Boards in February 2002. 


\subsection{Health needs assessments by DHBs}

DHBs took two different approaches to HNAs. Nine DHBs decided to undertake their own HNAs individually. Meanwhile 12 DHBs joined together in a shared service arrangement known as the Regional Health Needs Assessment Project (RHNAP), and took the collective approach to needs assessment described below.

\subsubsection{The Regional Health Needs Assessment Project (RHNAP)}

The Regional HNA Project (RHNAP) originally grew from the work of Good Health Wanganui (a Hospital and Health Service), which completed in 2000 a 'first phase' HNA jointly with the Wanganui District Council, (Good Health Wanganui, 2000b). Subsequently, six provincial hospitals, ${ }^{36}$ including Good Health Wanganui, contracted the Public Health Consultancy of the Wellington School of Medicine and Health Sciences to conduct a HNA for each of their DHB areas. As the project gained momentum, six other DHBs joined to make a total of $12,{ }^{37}$ covering approximately one third of the New Zealand population. These DHBs commonly had thinly spread populations, especially in their rural areas. The objectives of the RHNAP were:

- $\quad$ To produce relevant and accurate information in a HNA document that can be used for the strategic planning and decision-making process at a local DHB level.

- To develop a 'standard' (repeatable) methodology for doing DHB needs assessments.

- To focus primarily on the main population determinants of health, that is social, economic and cultural factors, with the aim of reducing social and economic inequalities in health.

- $\quad$ To carry out the assessment with the involvement of Mãori at all levels, so that the final document reflects Mãori needs, as defined by Māori.

- To identify gaps in the availability of data and in the capability of providers to supply information relevant to HNA.

\footnotetext{
${ }^{36}$ The term 'Provincial Hospitals' as distinct from metropolitan hospitals appears to relate in the New Zealand context to the New Zealand Provincial Hospital Group of 10 secondary provincial hospitals and health services formed in 1999 to promote common interests from their mainly 180-250 bed hospitals.

${ }^{37}$ The 12 DHBs that participated in the shared service arrangement were: Northland, Tairawhiti, Lakes, Taranaki, Wanganui, Hawke's Bay, MidCentral, Wairarapa, Nelson-Marlborough, West Coast, South Canterbury, and Southland.
} 
- To ensure that the needs assessment be used as a vehicle for consultation and participation with the community and providers, including Māori and Pacific providers. (Public Health Consultancy, 2001, p.4)

The project was set up in advance of the new legislation (Minister of Health, 2000e), as the organisations involved realised the value of gathering information on health needs ahead of time to prepare for decision-making. The rationale for a shared services arrangement for HNA was that scarce resources (experts, tools, information, funding) could be shared, standardised methodology could be used, and useful comparisons could be made between DHBs.

The RHNAP was sponsored by the Public Health Consultancy at the Wellington Clinical School in collaboration with Te Roopu Rangahau Hauora a Eru Pomare (Māori research unit). The project included a Research Advisory Group to provide oversight and direction for the project, advice on methodology, data analysis and interpretation, and critical review of reports (see Figure 12). The Research Advisory Group included Dr Cindy Kiro (social policy), Associate Professor Philippa Howden-Chapman (health and health policy), Dr Tony Blakely (public health physician and epidemiologist), Dr Tim Rochford (Māori health), Des O'Dea (health economist), Dr Fran McGrath (public health physician), Professor Alistair Woodward (epidemiologist), Associate Professor Richard Morgan (geography), and Dr Paul Callister (economist). The Ministry of Health guidelines were used as the basis for the project (Ministry of Health, 2000b).

The Project Co-ordinator worked closely with the Project Steering Group whose members were drawn from the participating DHBs. Each of the DHBs formed a Local Reference Group of between 12 and 25 people with an interest in health, including Māori. Their role was to support the project, ensure local community engagement, promote Māori input and participation, facilitate access to local people and information sources, raise issues with the Project Steering Group, and critique the final report. 


\section{Figure 12: Project Structure of the Regional HNA Project (RHNAP)}

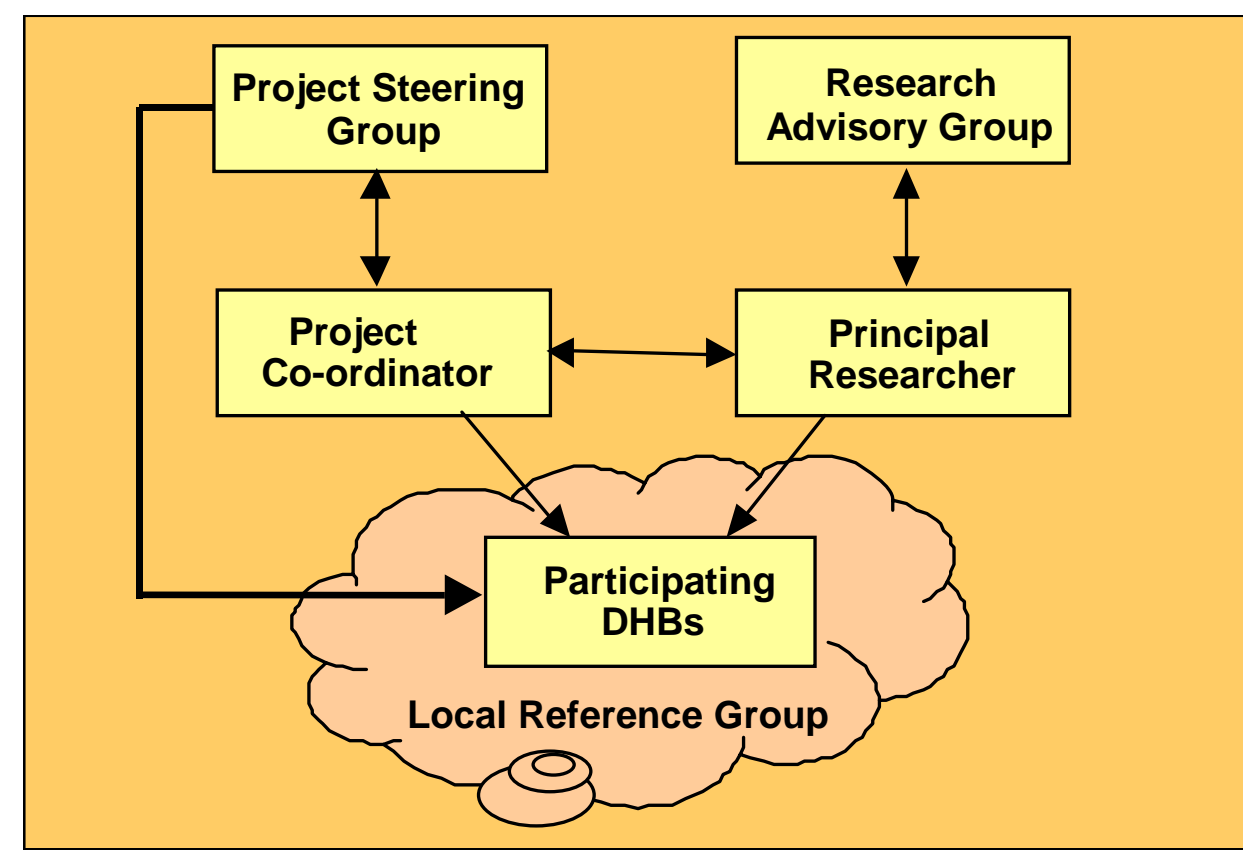

Source: Regional Health Needs Assessment Project, 2001

The definition of HNA adopted by the RHNAP was 'Systematic research effort to gain detailed understanding of the health needs of a population residing in a defined geographical area.' It was considered that a broad definition of health, including socioeconomic determinants of health, should be used, and that needs resulted from health status. The HNAs took account of demographic data, and data on socio-economic status, risk behaviours, health status, and services provision, besides summarising the views of community and 'experts'. Priorities for health service delivery were recommended for each DHB.

Various national databases were used, including 1996 Census data, hospitalisation data, national health surveys, existing reports such as RHAs' needs assessments, local data such as primary care utilisation rates, and qualitative data, in the form of provider surveys, key informant interviews and focus groups. The Research Group identified gaps in knowledge related especially to ethnicity, primary care, Māori health need, and mental health, and noted that demographic data needed updating using Census 2001 data. The outputs and outcomes of the project were: technical and summary reports for 
each DHB; transfer of skills and knowledge to DHBs; a platform for further collaborative work; identification of commonalities; and an impact on DHB decisionmaking. The place of the RHNAP relative to DHBs' responsibilities is illustrated in Figure 13.

Figure 13: District Health Board responsibilities and the district health board planning cycle $^{38}$

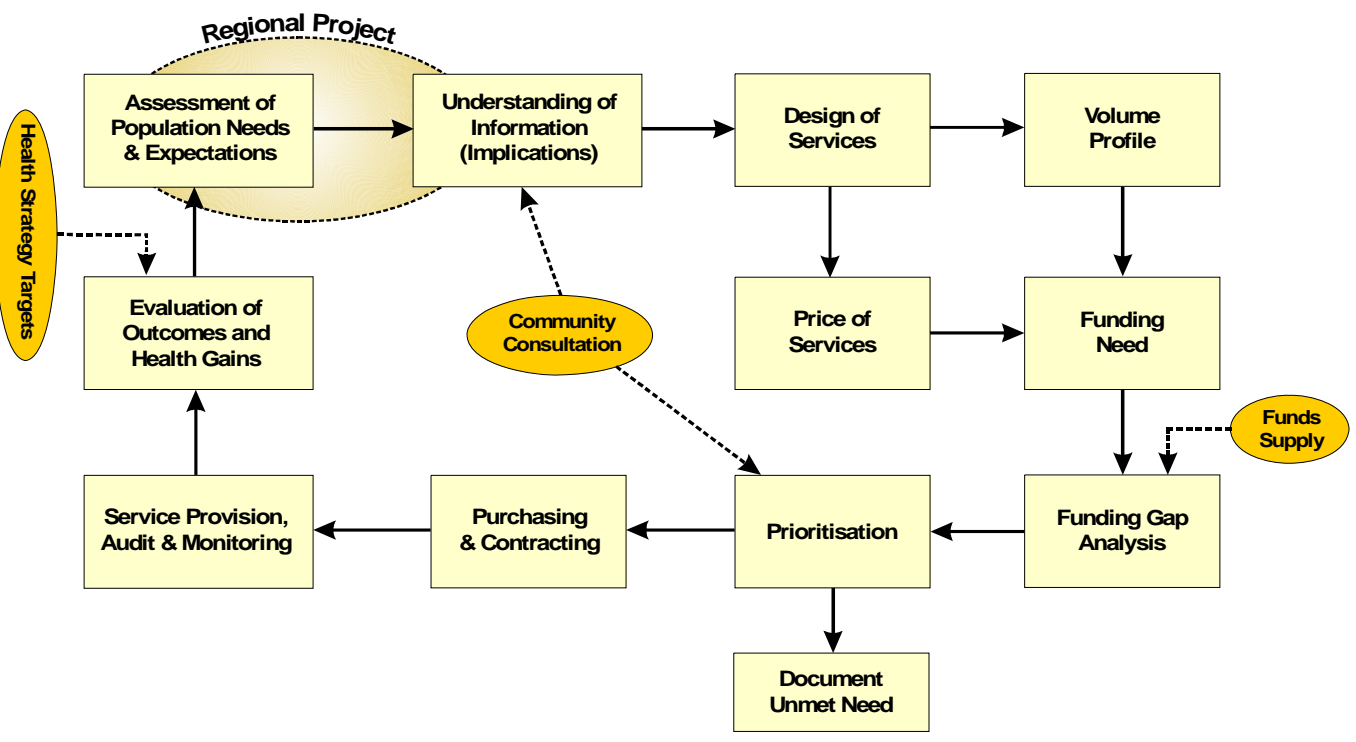

The RHNAP research team also presented its research method and findings to the Health Services Research and Policy Conference held in Wellington in December 2001 (Mitchell, Howden-Chapman, Bolevich and Smith, 2001).

\subsubsection{Health needs assessments by other DHBs}

Auckland, Waitemata, and Counties Manukau DHBs collaborated, using the Northern Districts Shared Services Agency, to establish some of the principles for HNA and prioritisation, and to collect some data jointly. However, the Counties Manukau DHB

\footnotetext{
38 The use of this figure has been kindly agreed to by its author, Zoran Bolevich, Technical Advisory
} Services, 2000. 
subsequently published its health profile independently. The Auckland and Waitemata DHBs used the Agency to collect most of their data and then each published their own report. The Hutt DHB needs assessment is discussed as a Case study in Section 7.8.1. These DHBs mostly used their own staff to gather and analyse data for their HNAs.

\subsection{DHB health needs assessment document analysis}

\section{Meaning of need}

Two thirds of the DHBs (12 of which were in the RHNAP) briefly discussed the meaning of need in published HNA reports. For most, need was formulated at a fairly high level, in terms of Bradshaw's typology of need, as 'capacity to benefit'. It was noted that views on health need vary between individuals, groups and cultures, as need is a value-laden concept. The RHNAP referred to all of Bradshaw's concepts of need: normative, expressed, comparative, and felt needs. Normative need was described as that defined by experts (for example, the 13 priority population health objectives set out in the New Zealand Health Strategy). Expressed need was defined as 'what can be inferred about need from observing how people use services (so measurement of services and their utilisation is (sic) taken to be an indicator of expressed need or demand).' Comparative need 'infers that the needs arising in one location can be deemed to be similar to those in another location if people have the same sociodemographic characteristics (measured by inter-regional comparisons).' Felt need was described in terms of 'what residents in a location say is need, problem or concern for them (measured by qualitative and social research approaches).' (Public Health Consultancy: Wellington School of Medicine and Health Sciences, 2001, p.5). DHBs noted the importance of looking beyond the indicators of health status in assessing health need, and considering wholeness and well-being. The socio-economic determinants of health were also to be considered predictors of levels of health and illness. 
DHBs noted that Māori concepts of health are holistic, with spiritual, emotional, social and bodily dimensions. As health was considered a tāonga, or treasure, under the Treaty of Waitangi, loss of protection and control resulting from breaches of the Treaty in turn was suggested to have resulted in much of the current poor state of Māori health. For Māori health needs to be met, health need had to be interpreted to include the need for Māori to regain control over the factors that influence their health. Only if those requirements were met could an improvement in Māori health status be expected.

In summary, DHB discussions regarding the meaning of need were brief, and all 12 DHBs in the RHNAP project used the same generic material within their HNAs. DHBs did not discuss the meaning of need to any significant extent, and not at all in one third of cases.

\section{Evaluation of quality of DHB HNAs}

The quality of DHB HNAs was of interest as these HNAs were the datasets upon which DHBs were supposedly basing their planning decisions. To evaluate the quality of HNAs, it was first necessary to specify a 'gold standard' against which to measure them. This took the form of a standard, set and made available by the Ministry of Health, to which were added additional criteria derived from international best practice. The Ministry of Health set the standard for DHB HNA with the Operational Policy Framework $^{39}$ 2001/2002, Accountability Indicator GOV-02 Effective Health Needs Assessment, shown in Box 5.

\footnotetext{
39 The Operational Policy Framework is provided to DHBs each year by the Ministry of Health and sets out the operational policies under which they must act. Accountability Indicators refer to specific performance measures that must be met by DHBs.
} 


\section{Box 5: Effective HNA Accountability Indicator for DHBs in $01 / 02$}

\section{GOV-02 Effective Health Needs Assessment}

\section{Objective}

A comprehensive health needs assessment report is produced for the DHB population. The report is to be provided to the Ministry of Health and is to be completed by 1 November 2001.

\section{Measure}

A health needs assessment report (which covers the first two sections of the document Health Needs Assessment for New Zealand: An Overview and Guide, December 2000) is completed by 1 November 2001 for the DHB population which:

- Is consistent with the approach in Health Needs Assessment for New Zealand: An Overview and Guide, December 2000.

- Gives particular attention to the New Zealand Health Strategy Population Health Priorities.

- Analyses the distribution of diseases, environmental factors and their determinants across their population to identify those groups (which may include Māori, Pacific people and people in lower socio-economic groups) experiencing poorer health outcomes.

- Lists the providers, services and numbers of patients receiving services from those providers again giving attention to New Zealand Health Strategy priorities.

- Considers a range of evidence informed interventions including public health measures, actions on the wider determinants of health and health care services.

- Involves participation and appropriate targeted consultation with groups within populations they are responsible for including local Iwi/Māori and where appropriate Pacific communities in respect to the health needs assessment report process.

- Involves mainstream services provided to Māori (and where appropriate Pacific communities) and services provided by Māori health providers (and where appropriate Pacific providers) are captured in the health needs assessment report.

\section{Frequency}

By 1 November 2001.

Source: Ministry of Health, 2001

The accountability requirements for DHB HNAs set out in this accountability indicator were used as the basis for evaluation; and a set of review indicators against which to assess the quality of the needs assessments was developed. The review criteria template, (see Appendix 14, p.332) is in two parts. Part A uses indicators based directly on the Effective HNA Accountability Indicator for DHBs, with a number of review criteria developed for each indicator. Part B includes additional criteria known to be important in needs assessments, such as stakeholder involvement, statement of methodology, 
presence of all significant data (including primary care and mental health data), and the making of recommendations. A separate criterion, the discussion of the meaning of need, was also included, as DHBs could be expected to formulate their interpretation of this pivotal concept. The final set of 23 review criteria was then used as the basis of an evaluation of each of the DHB population HNAs. Each review criterion was allocated a score of 2 if it was met, 1 if partially met, and 0 if it was not met. The needs assessment scores were then added to give an overall quality score for each DHB HNA.

The following table reports the overall Quality scores for individual DHB HNAs. Although the 12 regional DHBs all used the central agency at the Wellington Clinical School, each of these HNAs was individually evaluated. All scored equally at 44 points, and the HNAs differed only in their content. They have been grouped together as 'Regional DHBs' for reporting purposes. 
Table 24: Quality Scores for DHB HNAs

\begin{tabular}{|c|c|c|c|c|c|c|c|c|c|c|c|c|c|c|c|c|c|c|c|c|c|c|c|c|c|}
\hline DHB & 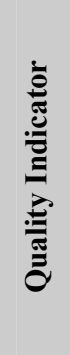 & $\begin{array}{l}* \\
0 \\
0 \\
. \\
0 \\
0 \\
0 \\
0\end{array}$ & 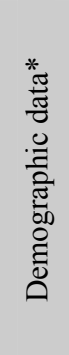 & 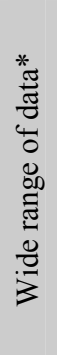 & 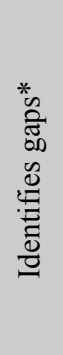 & 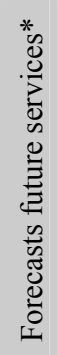 & 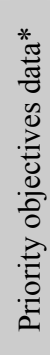 & 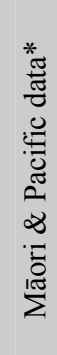 & 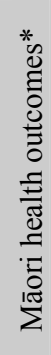 & 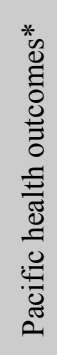 & 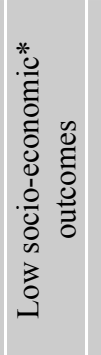 & 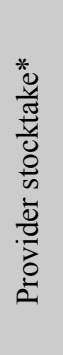 & 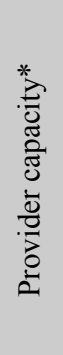 & 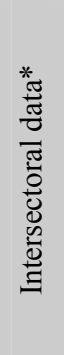 & 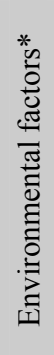 & 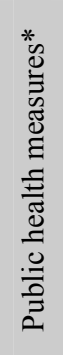 & 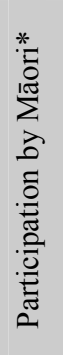 & 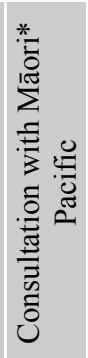 & 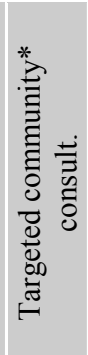 & 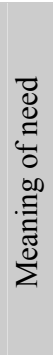 & 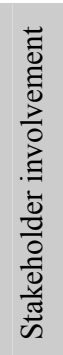 & 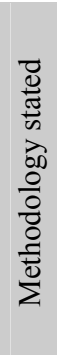 & 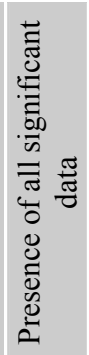 & 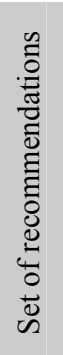 & 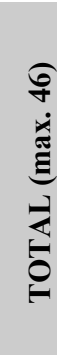 \\
\hline Regional DHBs (12) & & 2 & 2 & 2 & 2 & 1 & 2 & 2 & 2 & 2 & 2 & 2 & 2 & 2 & 2 & 2 & 2 & 2 & 2 & 2 & 2 & 2 & 1 & 2 & 44 \\
\hline Waitemata & & 1 & 2 & 2 & 2 & 2 & 2 & 2 & 2 & 2 & 2 & 2 & 2 & 2 & 2 & 2 & 1 & 1 & 1 & 2 & 0 & 2 & 1 & 2 & 39 \\
\hline Hutt & & 1 & 2 & 1 & 2 & 1 & 2 & 2 & 2 & 2 & 2 & 1 & 2 & 1 & 1 & 1 & 2 & 2 & 2 & 2 & 2 & 2 & 2 & 2 & 39 \\
\hline Auckland & & 1 & 2 & 2 & 2 & 2 & 2 & 2 & 2 & 2 & 2 & 2 & 2 & 2 & 2 & 2 & 1 & 2 & 2 & 0 & 1 & 1 & 1 & 2 & 39 \\
\hline Otago & & 0 & 2 & 2 & 2 & 1 & 2 & 2 & 2 & 2 & 2 & 1 & 1 & 1 & 2 & 2 & 1 & 0 & 2 & 0 & 0 & 1 & 1 & 0 & 29 \\
\hline Counties Manukau & & 0 & 2 & 2 & 2 & 2 & 2 & 2 & 2 & 2 & 2 & 0 & 0 & 0 & 2 & 2 & 0 & 0 & 0 & 0 & 0 & 2 & 1 & 1 & 26 \\
\hline Bay of Plenty & & 1 & 1 & 2 & 2 & 1 & 2 & 2 & 2 & 2 & 2 & 0 & 0 & 0 & 0 & 0 & 0 & 0 & 0 & 0 & 0 & 0 & 1 & 2 & 20 \\
\hline Canterbury & & 0 & 1 & 1 & 2 & 1 & 2 & 2 & 1 & 1 & 0 & 2 & 1 & 0 & 2 & 0 & 0 & 0 & 0 & 0 & 0 & 0 & 2 & 0 & 18 \\
\hline Capital and Coast & & 1 & 2 & 2 & 1 & 0 & 1 & 2 & 1 & 1 & 1 & 0 & 1 & 0 & 1 & 0 & 0 & 0 & 0 & 0 & 0 & 2 & 1 & 0 & 17 \\
\hline
\end{tabular}

Note: Review criteria scores: Criteria not met $=0$; Criteria partly met $=1$; Criteria met $=2$. Maximum possible score $=46 . *=$ minimum requirement of the Ministry of Health set out in the

accountability indicator. The following Regional DHBs participated in the Regional HNA process involving the Wellington Clinical School as a central agency: Northland, Lakes,

Tairawhiti, Taranaki, Whanganui, MidCentral, Wairarapa, Nelson Marlborough, West Coast, South Canterbury, Otago, and Southland. 
The DHBs are ranked according to the Quality Score for HNAs. The Regional DHBs performed better than all the others, with Waitemata, Hutt and Auckland coming close on performance; but surprisingly Bay of Plenty, Canterbury, and Capital and Coast achieved less than half of the maximum Quality Score. The conclusion is that the centrally-driven RHNAP process resulted in health need assessments of better quality than those performed locally by DHBs. As will be seen later however, quality is only one pertinent factor; and other considerations have a greater influence on the ability of boards to achieve connection between HNAs and prioritisation, planning and purchasing.

\section{DHB population health needs and needs for services}

To understand the range and frequency of health needs reported by DHBs, HNA documents were analysed for the key health needs, and needs for services, identified by assessors. In most cases these needs were identified within the documents themselves, but were sometimes recorded in the DAPs.

Some HNAs had to be searched closely to find statements of key health needs and requirements for services. It was much easier to read other HNAs and obtain from them clear recommendations regarding the need for health services. Most HNAs were collections of epidemiological data amounting to a health profile, with little analysis of key problems, and several contained no recommendations at all, although priorities could be determined on close examination.

In addition, HNAs were influenced in their data collection and analysis by the list of government priority health objectives, rather than collecting data de novo. It could be argued that this was a desirable outcome, as DHBs were focusing their efforts in directions required by government. It could also be argued that if government has set the priority objectives correctly, these would feature heavily in HNAs anyway. 
Table 25 tabulates health needs and needs for services by DHB. ${ }^{40}$ Table 26 ranks key health needs and services identified as priorities by DHBs. Almost all boards have recorded significant health need in the government priority areas. This finding suggests either that government priority population health objectives have had a significant impact on the recording of HNAs, or that the government in fact accurately predicted population-based health needs and prioritised accordingly. If the latter is true, it can be argued that HNAs had no value in determining priority health objectives because the government had already done it, and got it 'right'. However, other needs and requirements for services were identified beyond the priority objectives, and the HNAs have therefore provided additional local information, specific to individual boards.

Among other reported health needs and needs for services, Māori health, primary care, rural health, primary/secondary interface, water quality, and health information stand out for the majority of boards. Whilst the first four were not surprising, the last two were. Primary health care is a key focus for the government and the importance attached by the Minister of Health to the Primary Care Strategy is indicative of Government's commitment to the sector. Boards were asked to include environmental health needs in the needs assessments (Ministry of Health, 2000b), and water quality was clearly a significant concern. Therefore it was surprising that not one board rated addressing water quality highly in DAPs. The need to obtain better data, particularly from primary care, was frequently expressed as a need. This is consistent with the observations of the Controller and Auditor-General regarding the quality of primary care data, and the requirement to address this concern:

The arrangements for purchasing primary care are not based on a comprehensive assessment of health needs, and, in our view, fall well short of what is needed to ensure the provision of effective and efficient services (Controller and AuditorGeneral, 2002).

${ }^{40}$ Individual DHB data are also recorded in Appendix 13, p.322 along with the level of importance attached to each item by DHBs in their prioritisation process by boards. 
Table 25: Key health needs and need for services identified by District Health Boards in HNAs

\begin{tabular}{|c|c|c|c|c|c|c|c|c|c|c|c|c|c|c|c|c|c|c|c|c|c|c|c|c|c|c|c|c|c|c|c|c|c|c|c|c|c|c|c|c|c|}
\hline DHB & 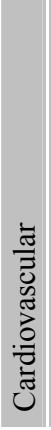 & 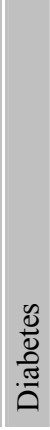 & 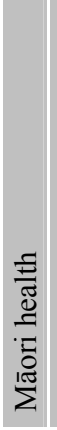 & 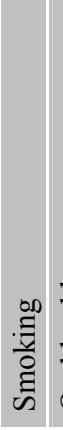 & 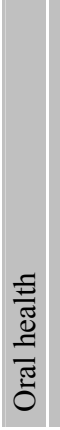 & 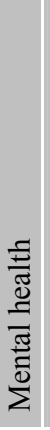 & 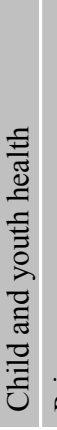 & 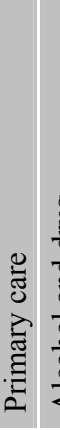 & $\begin{array}{l}\frac{00}{2} \\
\vec{y} \\
\overline{0} \\
\bar{\sigma} \\
\overline{0} \\
\frac{0}{0} \\
\frac{0}{4}\end{array}$ & $\begin{array}{l}\frac{0}{0} \\
: \frac{0}{\Xi} \\
\tilde{n}\end{array}$ & 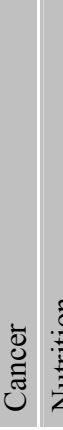 & 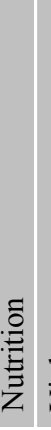 & $\begin{array}{l}0 \\
\frac{0}{0} \\
\frac{0}{0} \\
\\
>\end{array}$ & $\begin{array}{c}2 \\
0 \\
0 \\
0 \\
0\end{array}$ & 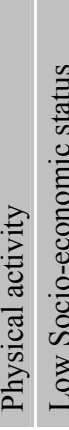 & 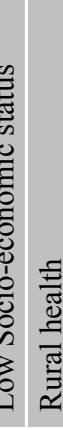 & 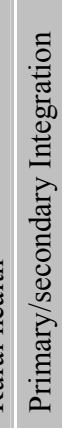 & & 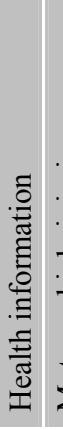 & 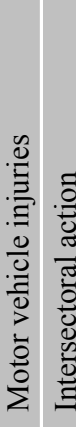 & 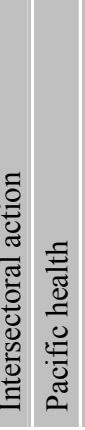 & 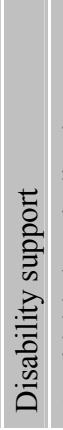 & 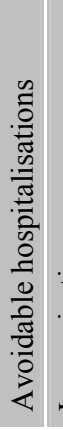 & 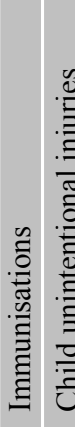 & 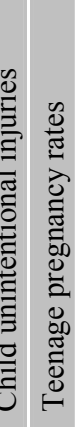 & 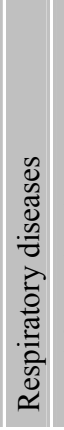 & 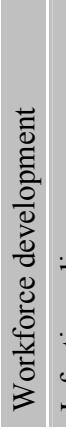 & 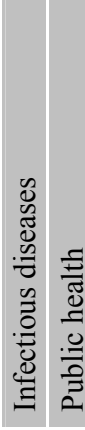 & 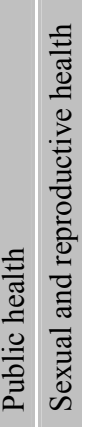 & 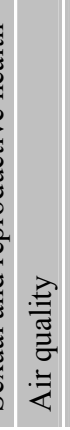 & $\frac{\tilde{\Xi}}{\stackrel{0}{0}}$ & 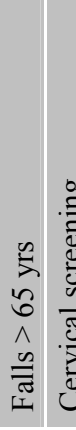 & 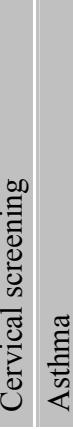 & 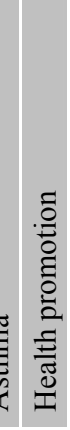 & 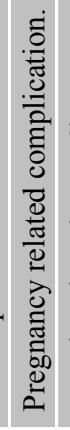 & 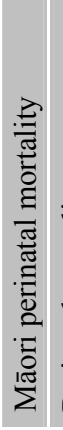 & 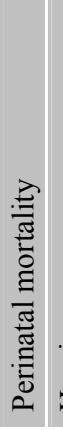 & 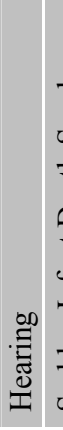 & 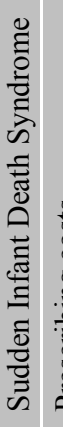 & 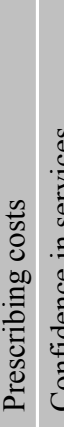 & 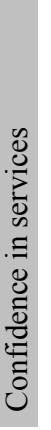 \\
\hline Northland & $*$ & * & $*$ & $*$ & $*$ & $*$ & $*$ & * & * & $*$ & * & $*$ & $*$ & * & & * $\quad *$ & * & $*$ & & & $*$ & $*$ & $*$ & & * & & & & * & & & & * & & & & & & & & \\
\hline Waitemata & $*$ & * & $*$ & $*$ & * & $*$ & * & * & * & $*$ & * & * & * & * & $* \quad *$ & * & * & $*$ & $*$ & * & * & & & $*$ & & & & $*$ & & $*$ & & & & & & & & & & & \\
\hline Auckland & $*$ & * & $*$ & $*$ & * & $*$ & & * & * & $*$ & * & * & * & & * & * & * & & & & * & * & * & * & & * & & $* \quad *$ & * $\quad *$ & & * & & & & & & & & & & \\
\hline Counties Manukau & $*$ & * & $*$ & & * & & $*$ & $*$ & $*$ & & & & & & & & & & & & * & & & & & $*$ & & $* \quad *$ & * & & * & & & & & & & & & & \\
\hline Lakes & $*$ & $*$ & $*$ & $*$ & $*$ & $*$ & $*$ & $*$ & $*$ & $*$ & $*$ & $*$ & $*$ & $*$ & $* \quad *$ & * & & $*$ & $*$ & $* \quad *$ & * & & & & * $*$ & & $*$ & $*$ & & & $*$ & & & & & $*$ & $*$ & & & & \\
\hline Bay of Plenty & $*$ & * & * & $*$ & * & * & * & & * & $*$ & * & * & $*$ & * & $* \quad *$ & * & & & & * & & & & $* \quad *$ & * $*$ & * & & * & * & & & * & & & & & & & * & & \\
\hline Tairawhiti & $*$ & $*$ & $*$ & $*$ & $*$ & $*$ & $*$ & $*$ & $*$ & $*$ & * & $*$ & $*$ & $*$ & * & * & & & $*$ & $*$ & & & * & $* \quad *$ & $* \quad *$ & & $*$ & & & & & $* *$ & * $*$ & k & $*$ & & & & & & \\
\hline Taranaki & $*$ & $*$ & $*$ & $*$ & & $*$ & & $*$ & $*$ & $*$ & $*$ & * & $*$ & $*$ & * & * & $*$ & & $*$ & $* \quad *$ & * & & & & $*$ & $*$ & * & & $* \quad *$ & & & $* \quad *$ & * & & & & & $*$ & & & \\
\hline Hawkes Bay & $*$ & * & * & * & $*$ & $*$ & * & * & * & * & * & * & $*$ & * & & * $\quad *$ & $*$ & & $*$ & & * & & * & $* \quad *$ & * $\quad *$ & & * & & * & & & & $*$ & * $*$ & & & * & $*$ & & & * \\
\hline Whanganui & $*$ & $*$ & & $*$ & $*$ & $*$ & $*$ & & $*$ & & $*$ & $*$ & & $*$ & $*$ & & & $*$ & & & & & & & * & $*$ & & & & & & & & & & & & & & & \\
\hline MidCentral & $*$ & $*$ & $*$ & $*$ & $*$ & $*$ & $*$ & $*$ & $*$ & $*$ & $*$ & $*$ & $*$ & $*$ & $*$ & * & $*$ & $*$ & & & & $*$ & * & & & & & & & $*$ & & & & & & & & & & & \\
\hline Hutt & $*$ & * & * & & & $*$ & * & * & & & * & & & & & & & & & & * & * & & & & * & & & * & & * & & & * & & & & & & & \\
\hline Capital and Coast & $*$ & $*$ & $*$ & $*$ & $*$ & * & $*$ & & & $*$ & & & & & & & & & & & $*$ & $*$ & & & & & & & & & & & & & & & & & & & \\
\hline Wairarapa & $*$ & * & * & $*$ & * & * & $*$ & $*$ & $*$ & $*$ & * & $*$ & $*$ & * & $* \quad *$ & * $\quad *$ & * & $*$ & $*$ & $* \quad *$ & * & & * & $*$ & * $*$ & & * & & & & & $* \quad *$ & * $*$ & * & $*$ & * & & & & & \\
\hline Nelson Marlborough & & $*$ & $*$ & * & $*$ & * & $*$ & $*$ & $*$ & & & $*$ & $*$ & & $* \quad *$ & * $*$ & $*$ & $*$ & $*$ & $* \quad *$ & * & * & * & $*$ & & & & & & * & & & & & & & & & & & \\
\hline West Coast & $*$ & $*$ & $*$ & $*$ & $*$ & & $*$ & $*$ & $*$ & * & $*$ & & & & * & * $*$ & $*$ & $*$ & $*$ & $* \quad *$ & * & $*$ & * & $*$ & & $*$ & & & & & & & & & & & & & & & \\
\hline Canterbury & $*$ & * & * & $*$ & * & * & * & $*$ & * & $*$ & * & * & $*$ & * & * & * & & & & & $*$ & & & & * & & & & & $*$ & * & & & * & & & & & & & \\
\hline South Canterbury & $*$ & $*$ & $*$ & $*$ & $*$ & $*$ & & $*$ & & $*$ & & & & & * & * $\quad *$ & & $*$ & $*$ & $*$ & & & & & & & * & $*$ & & $*$ & & & & & & & & & & $*$ & \\
\hline Otago & $*$ & $*$ & $*$ & $*$ & $*$ & * & $*$ & $*$ & $*$ & $*$ & $*$ & $*$ & $*$ & $*$ & $*$ & & $*$ & & & * & $* *$ & $*$ & & & & & & & & & & & & & & & & & & & \\
\hline Southland & $*$ & & * & * & * & * & * & * & * & $*$ & * & * & * & * & $* \quad *$ & * $*$ & * & * & * & * & $* \quad *$ & & & & & & & & * & & & & & & & & & & & & \\
\hline TOTAL & $\begin{array}{l}1 \\
9\end{array}$ & $\begin{array}{l}1 \\
9\end{array}$ & $\begin{array}{l}1 \\
9\end{array}$ & $\begin{array}{l}1 \\
8\end{array}$ & $\begin{array}{l}1 \\
8\end{array}$ & $\begin{array}{l}1 \\
8\end{array}$ & $\begin{array}{l}1 \\
7\end{array}$ & $\begin{array}{l}1 \\
7\end{array}$ & $\begin{array}{l}1 \\
7\end{array}$ & $\begin{array}{l}1 \\
6\end{array}$ & $\begin{array}{ll}1 & 1 \\
6 & 5\end{array}$ & $\begin{array}{l}1 \\
5\end{array}$ & $\begin{array}{l}1 \\
4\end{array}$ & $\begin{array}{ll}1 & 1 \\
3 & 2\end{array}$ & \begin{tabular}{l|l}
1 & 1 \\
2 & 1
\end{tabular} & $\begin{array}{ll}1 & 1 \\
1 & 1\end{array}$ & $\begin{array}{l}1 \\
1\end{array}$ & \begin{tabular}{|l|}
1 \\
0
\end{tabular} & $\begin{array}{l}1 \\
0\end{array}$ & \begin{tabular}{l|l}
9 & 9
\end{tabular} & \begin{tabular}{l|l}
9 & 8
\end{tabular} & 8 & 8 & \begin{tabular}{l|l}
8 & 7
\end{tabular} & $\begin{array}{ll}7 & 7\end{array}$ & 7 & 6 & & & 5 & 5 & \begin{tabular}{l|l}
4 & 4
\end{tabular} & & & & & & & & & \\
\hline
\end{tabular}


Table 26: Ranked frequency of key health needs and services identified by DHBs as priorities

\begin{tabular}{|l|c|l|c|l|c|}
\hline Health need or service & Rank & Health need or service & Rank & Health need or service & Rank \\
\hline Cardiovascular* & 1 & Low socio-economic status & 9 & Infectious diseases & 14 \\
\hline Diabetes* & 1 & Rural health & 9 & Public health & 15 \\
\hline Māori health & 1 & Primary/secondary integration & 9 & Sexual and reproductive health & 15 \\
\hline Smoking* & 2 & Water quality & 10 & Air quality & 15 \\
\hline Oral health* & 2 & Health information & 10 & Electives & 15 \\
\hline Mental health* & 2 & Motor vehicle injuries & 11 & Falls $>65 y r s$ & 16 \\
\hline Child and youth health* & 3 & Intersectoral action & 11 & Cervical screening & 16 \\
\hline Primary care & 3 & Pacific health & 12 & Asthma & 16 \\
\hline Alcohol and drug* & 3 & Disability support & 12 & Health promotion & 17 \\
\hline Suicide* & 4 & Avoidable hospitalisations & 12 & $\begin{array}{l}\text { Pregnancy related } \\
\text { complications }\end{array}$ & 18 \\
\hline Cancer* & 4 & Immunisations & 12 & Māori perinatal mortality & 18 \\
\hline Nutrition* & 5 & Child unintentional injuries & 13 & Perinatal mortality & 18 \\
\hline Violence* & 6 & Teenage pregnancy rates & 13 & Hearing & 18 \\
\hline Obesity* & 7 & Respiratory diseases & 13 & Sudden Infant Death Syndrome & 19 \\
\hline Physical activity* & 8 & Workforce development & 14 & Prescribing costs & 19 \\
\hline & & & & Confidence in services & 19 \\
\hline
\end{tabular}

Note: Items marked * are the 13 government priority population health objectives set out in the New Zealand Health Strategy.

\subsection{Prioritisation}

Prioritisation is concerned with how we make decisions about what health and disability services or interventions to fund, for the benefit of New Zealanders, within the resources available. (Health Funding Authority, 2000d, p.6)

The history of the prioritisation of health services in New Zealand has been discussed in depth in the literature review (Chapter 2.4, p.38). Prioritisation is necessary to maximise the health of the population, reduce inequalities in health status, and meet health care needs or requirements for services or interventions, within existing resources. Prioritisation decisions involve determining what changes should be made to the current mix of services, interventions, or programmes. In practice, prioritisation focuses on effectively managing what has been purchased (existing services), purchasing new services (new investment), and reallocating funds to alternative (higher priority) services (disinvestments and reprioritisation). Further discourse on the nature of prioritisation is not appropriate here, but the context of prioritisation by DHBs, 
prioritisation frameworks adopted by DHBs and how HNA links into those frameworks are all pertinent.

\subsubsection{Context of prioritisation by DHBs}

'The New Zealand Health Strategy provides the framework within which District Health Boards and other organisations across the health sector will operate.' (Minister of Health, 2000d,p.iii) Priorities for the New Zealand health sector were already set by the 13 priority population health objectives specified in the New Zealand Health Strategy (p.13). The New Zealand Disability Strategy sets out the objectives for making New Zealand more inclusive for disabled people (Minister of Health, 2001b, p.11). It provides objectives related to disability support services, and covers such areas as education, human rights, employment and economic development. Three other key documents are the Primary Health Care Strategy, Māori Health Strategy: He Korowai Oranga and the Pacific Health and Disability Action Plan (Minister of Health, 2001c, Minister of Health, 2001a, Minister of Health, 2002). The current policy environment is one of control by the government over the process and outcomes of prioritisation.

The DHB accountability measure GOV-03 is an operational requirement of the Ministry, and was included in DHB DAPs and Crown Funding Agreements with DHBs. Although this indicator has been included earlier, it is repeated here for completeness:

\section{Box 6: Prioritisation Accountability Indicator for DHBs in 02/03}

\section{GOV-03 Prioritisation}

Undertake a prioritisation round. Identify a list of funding options including planned sources of funding (which may include reprioritisation of current baseline expenditure) and provide the Ministry with a one page summary of the results by 31 May 2003. Include with the summary, documentation that shows that the prioritisation measure was met. 
The Ministry of Health publishes an Operational Policy Framework (OPF) each year, which provides quasi-regulatory and guideline material for DHBs, including information on governance and accountability, the financial operating environment, performance and monitoring, reporting and the Nationwide Service Framework. ${ }^{41}$ The OPF requires that DHBs will, in prioritising and decision-making:

- Collaborate with other DHBs in relation to regional and national services

- Use a principle-based framework that links directly to the principles of the New Zealand Health Strategy

- Through the development and implementation of the prioritisation process involve Māori, consider and respond to their needs, and support Māori capacity building

- Clearly document why decisions were made, who the decision-makers were, what the decision-making process was and how the community was involved in the decision-making. (Ministry of Health, 2002b)

The Ministry of Health issues the Service Coverage Schedule ${ }^{42}$ which states that the level of services to be funded (service mix) will be determined in line with the following requirements, to ensure that prioritisation decisions are made to extract maximum benefit from the funding available:

- Access to services will be determined on a fair and reasonable basis, and generally accepted clinical protocols.

- Priority for access will be granted on the basis of need, ability to benefit and/or an improved opportunity for independence for those with a disability. The responsible funder will, where appropriate, target delivery of services to those groups with poor health status and those likely to benefit.

- The responsible funder will ensure people have reasonable access to services as close as possible to where they live, taking into account the geographic location of where they live and the nature of the service to which access is required.

- When determining the availability of funded services, the responsible funder will consider and accommodate the needs of the people in remote areas in the

${ }^{41}$ The Nationwide Service Framework (NSF) is a collection of definitions, methodologies and processes that allow the sector to use common language when analysing, funding and monitoring services. The NSF includes (but is not limited to) decision-making/prioritisation processes, consultation guidelines, standard service agreement forms, defined service units, national service agreement monitoring and risk monitoring processes.

42 The Service Coverage Schedule (SCS) is a statement of the minimum range and standard of services that the government expects to be available for New Zealanders. It is provided to DHBs by the Ministry of Health. 
most practical, efficient and clinically safe way. (Service Coverage Schedule 2001/02)

Service coverage is included in the Crown Funding Agreement so as to allow the Minister to explicitly agree with DHBs the level of service coverage for which they are held accountable.

Ring-fencing is another mechanism used by government to control the allocation of funds to ensure that certain services are prioritised (see Section 2.4.7, p.48 ). Funding may also be 'tagged' for certain services, without the formal mechanism of ringfencing.

It is against this regulatory and quasi-regulatory framework that DHBs are required to establish and employ principles for prioritising health services. The key to the success of this process is how effectively DHBs link findings from the HNA process into prioritising services for funding. The tightly managed environment governed by the 13 priority population health objectives for DHBs sets a predetermined outcome for prioritisation.

\subsubsection{Prioritisation principles established by DHBs}

Various approaches have been taken to the prioritisation of health services, including those of the National Health Committee (see Chapter 2.4, p.38). The Ministry of Health used a consensus approach with an Expert Advisory Group to establish goals and objectives for priorities for inclusion in the New Zealand Health Strategy. The Advisory Group and the Ministry agreed on 13 priority population health objectives that both the Ministry and DHBs would work towards (Minister of Health, 2000d, p.13). Given the good progress of the HFA in advancing prioritisation (Health Funding Authority, 2000d, Health Funding Authority, 2000e), and that a number of DHBs employed former HFA staff who were well versed in the HFA prioritisation process, it is not surprising that the majority of DHBs adopted principles based on the previous HFA framework. Those principles were effectiveness, cost, equity, Māori health, and acceptability (see 
section 2.4.4, p.42). Analysis of the prioritisation principles chosen by DHBs indicates that a total of 36 different principles were selected by DHBs, with the average number of principles per board being 6.5 (range $4-11$ ). Of the 20 DHBs analysed, 10 included all five of the HFA principles, but only three exclusively so. Boards freely expanded on the range of HFA principles, and at least 10 boards omitted some or all of those principles. This demonstrates that boards were free to choose the principles by which they were to prioritise purchasing of health services. The interviews with Planning and Funding Managers, reported in the next section, will indicate whether the boards proceeded to apply the prioritisation frameworks with the new principles, or not. 


\section{Table 27: Prioritisation principles by DHB}

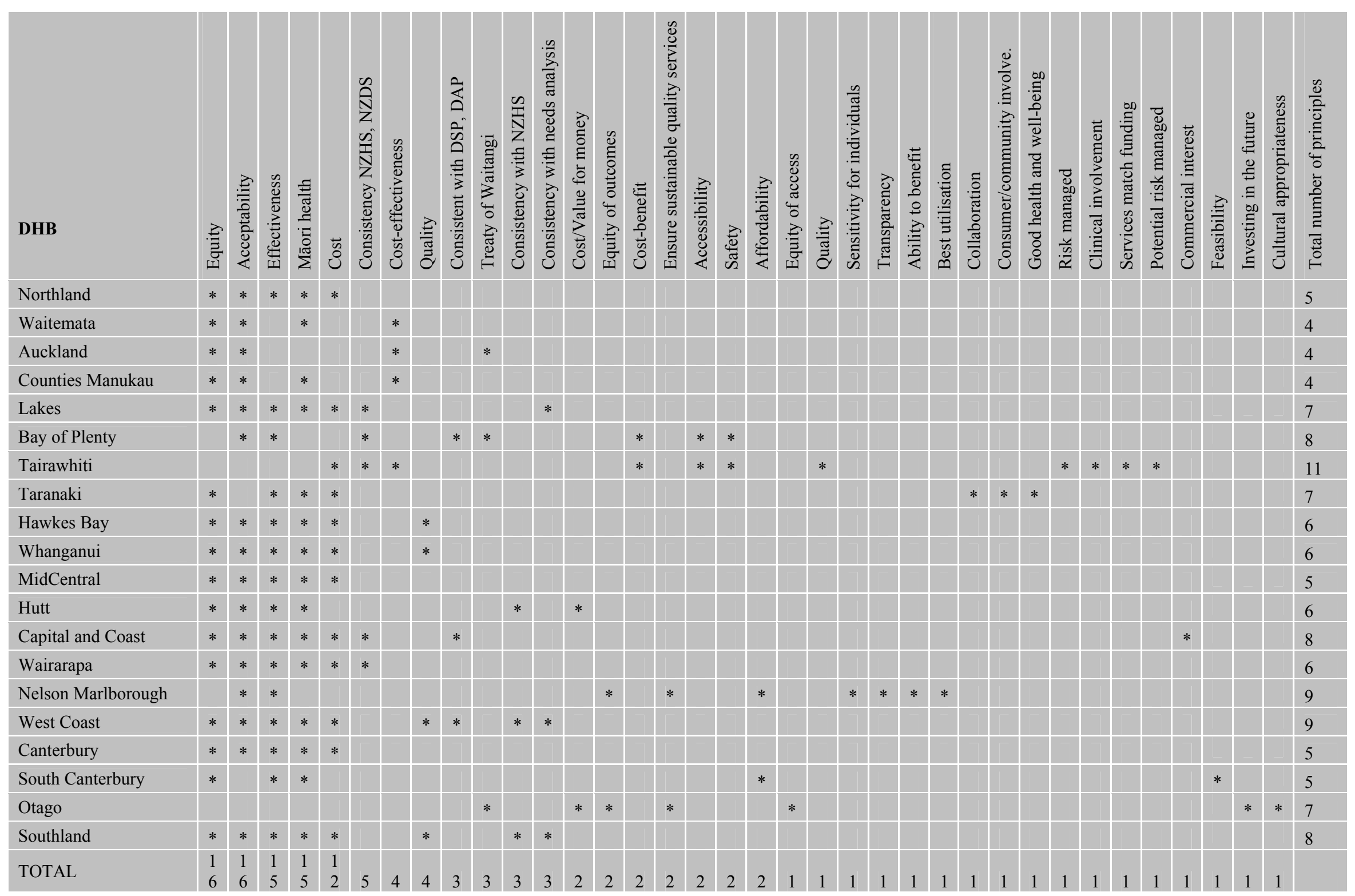




\subsection{Planning and Funding Manager interviews}

\subsubsection{Introduction}

DHB Planning and Funding Managers were interviewed to obtain their insights regarding the HNA process, District Strategic Plans (DSPs), District Annual Plans (DAPs), budgets, and purchasing.

The results are reported under the main headings of HNAs; prioritisation frameworks and prioritisation; key influences on DSPs; linkages between DAPs and budgets; and connections between HNAs and purchasing. Five case studies of DHBs are also presented to illustrate key differences between DHBs in the effectiveness of HNA in their planning processes.

\subsubsection{DHB health needs assessments}

Most DHBs found the HNA process to be valuable. The needs assessments brought together DHB demographic, epidemiological, primary care and provider stocktake data, alongside the views of the community, including Māori, on health services. Managers observed that the HNAs provided a health profile of populations within their districts, giving a snapshot of the health of these communities and a solid base of information on which to plan health services. Secondly, HNAs were considered to be useful baseline datasets for the future. Thirdly, although there was significant variation in the way that DHBs incorporated data into their planning processes, most DHBs felt that the HNAs contributed usefully to the planning process. As will be seen later, the data show that certain models of planning incorporated the analysis of health service requirements into planning better than others.

A few Boards took an ex post rather than ex ante approach to the use of HNA, and began the strategic planning process before HNA data became available. HNA data were used subsequently to validate the approach taken during the strategic planning 
process. Several other DHB managers commented that the HNA data confirmed their prior impressions regarding the health of their DHB population. Almost every DHB said that they intended using the HNA data to track the health status of the DHB population over time. Some used the HNA data to develop performance indicators to measure progress against health targets, in addition to those proposed by the Ministry of Health.

Managers reported that specific problem areas recurring in HNAs were violence and crime; respiratory infections and disease; diabetes; cardiovascular disease; transport; child and youth health; poverty; the poor health status of Māori; poor oral health; smoking; preventable injuries; and avoidable hospitalisations. Others noted a range of other issues such as 'poor health statistics' in the district; high socio-economic deprivation; and high accident rates. A few managers commented that the HNAs did not highlight any new key issues for them.

All DHBs involved in RHNAP found their Local Reference Group was a most valuable source of input to the HNAs. The DHBs conducted local surveys in their districts, using a standardised questionnaire prepared by the RHNAP. Most found the survey data useful, although several DHBs did not, and one thought that the questionnaire was poorly designed. DHBs involved with the project were also responsible for conducting 15-20 key informant interviews in their districts. All these DHBs found the process most useful for obtaining community views on the need for health services, including that of Māori and Pacific people. The DHBs also conducted interviews with community health providers and obtained useful data. Although the DHBs involved viewed the task as "huge, especially the local side," one commented that it "was work we probably needed to do in order to do the rest of our job." Smaller DHBs found the task difficult because of limited local capacity. The RHNAP sent each participating DHB a full Technical Report, a smaller Summary Report and a CD containing all their own data. Most DHBs subsequently placed their HNAs on their websites, with copies available directly from DHB offices. 
DHBs that conducted their own HNAs, ${ }^{43}$ all of them 'larger' DHBs, expressed no regrets about staying outside the provincial DHB process, and claimed a greater sense of 'ownership' of the data and process as a result. Most of them found internal staff to conduct their HNAs, while one or two used external contractors. Many of these DHBs formed steering committees for the HNAs, and most of those that did not wished they had done so (the exception was one whose HNA amounted to an epidemiological health profile).

DHBs involved in the RHNAP expressed considerable satisfaction regarding the process and outcomes of the project. Those involved considered that they had obtained high quality research data and relevant HNA reports, and gave the impression that most of them would use this process again. Shared services arrangements helped to relieve skill shortages and capacity problems. Many of the DHBs in the RHNAP reported that they would not have been able to conduct their own HNAs owing to their lack of skilled staff.

\section{Iwi relationships}

DHBs took various approaches to Māori health need. Many stated that Māori health was a priority for their Board. Others considered Māori health to be an 'overarching' or pervasive consideration. One DHB put it this way:

Māori have poor health and one of our overarching goals is to reduce disparities, so we didn't pick Māori health need as a priority on its own. We said it flowed through everything and within each of the strategies for everything else there has to be an objective to actually address Māori health needs.

Some DHBs found it difficult to obtain the participation and engagement of Māori, mainly because they did not already have a relationship with Iwi, and it took time to establish one. A number of DHBs said in retrospect that they should have engaged with Māori "earlier and better."

43 Waitemata, Auckland, Counties Manukau, Bay of Plenty, Waikato, Hutt, Capital and Coast, Canterbury, and Otago. 
Engagement with Māori groups varied from none to full consultation, according to managers. Some DHBs considered that involvement by Māori staff in the HNA was sufficient to obtain a Māori perspective. The Māori Health Units and managers and Māori members of boards, made an important contribution towards ensuring that a Māori voice was heard. In some boards, plans had to be signed off of by the Māori caucus, before being allowed to proceed to the Board. In one DHB, the Māori Committee was reported to be involved in all the decision-making, with a significant level of engagement. Some boards established Māori governance groups or formed partnership agreements. It appears that Māori participation and engagement with many DHBs was minimal. Māori considered that the needs assessments were not sufficiently in-depth, and a number of DHBs were now embarking on Māori HNAs.

\section{Pacific people}

Six DHBs recognised that they had a significant population of Pacific people. They saw consultation by means of fono ${ }^{44}$ as important, and Pacific staff members assisted in obtaining input from Pacific people. DHBs with a low representation of Pacific people could not easily identify Pacific community groups or Pacific providers. Consultation was therefore found to be more difficult, but was undertaken with individuals instead.

\section{Data issues}

Almost without exception, DHBs found it difficult to obtain and validate some types of data. The most difficult data to obtain were those from primary care and mental health services. Primary care data presented a challenge for almost every DHB, as Independent Practitioner Associations (IPAs) and GPs often would not release data. The data that DHBs obtained were generally of poor quality, so that in most cases it was described as unusable. Several DHBs reported "zero co-operation" from local GPs. Given the importance of primary care, and health prevention and promotion for health service delivery, DHBs found the virtual unavailability of primary care data most unsatisfactory. Some IPAs subsequently agreed that DHBs could access data. It was felt

\footnotetext{
${ }^{44}$ A 'fono' means a meeting. The word derives from the Samoan and Niuean languages.
} 
that Primary Health Organisations should in the future be required to produce data. Some DHBs suggested that it would be useful to obtain primary care data from national GP organisations. Mental health data were considered by all DHBs to be sparse, and many DHBs formed regional groups to obtain and analyse such mental health data as were available. Most of the data related to hospital discharges, and community mental health data were virtually unavailable. Some developments regarding collection of mental health data at national level were under way.

Immunisation data were also considered to be of poor quality. DHBs observed distinct differences between claims data held by the National Health Information Service (NZHIS) and data obtained from GPs. GPs' immunisation data were sometimes preferred, when available, as they were collected closer to the patient and considered to be more accurate.

All DHBs obtained good quality secondary care data from their hospitals and from the National Minimum Dataset (NMDS). Generally, data standardisation presented few problems, and where there were issues data were reworked to meet requirements. For example, Statistics New Zealand age bands, which did not match those of the NMDS, were reworked by a Ministry project was initiated for the purpose. Every DHB commented on the poor quality ethnicity information in datasets. This was viewed as a major hindrance, although few made any suggestions for improving ethnicity data collection.

Many DHBs commented that Census 1996 data used at the time of the HNAs were now out of date (Census 2001 data were released three months too late for the HNAs), and expressed a preference for having current data available in future. A number of DHBs said that they were now updating their data using the Census 2001 dataset. They noted that current demographic data would allow them to prepare more accurate projections in their three-year plans. Smaller DHBs noted that some of their data may be inaccurate because of small denominators, and the potential impact of small number changes on the numerator data. 
To obtain data on socio-economic determinants of health, managers held discussions with local Councils, CYFS, Police and the Fire Brigade. Interviewees commented that discussions regarding the exchange of data also frequently led to agreements about how DHBs and local councils would work together in the future.

\section{Timeframes}

Most DHBs found that the timeframe for completing the DHB HNA short. Some were able to start collecting data early, in one case a whole year ahead, and found that to have been beneficial. Because the task of data collection and collation was so big, most needed to allot additional personnel and resources to the project to complete their needs assessments by the Ministry of Health deadline of 1 November 2001. Although a period of 11 months was allowed from the commencement of the New Zealand Public Health and Disability Act 2000 on 1 January 2001 in which to complete HNAs, most DHBs found that in practical terms they had six months. The Ministry of Health reported that even then some DHBs did not complete their HNAs by the deadline.

\section{Community consultation}

Community consultation during the HNA process varied considerably, probably because of variables such as the philosophical approach of DHB boards and staff, and the availability of resources. DHBs were not required to consult the community during the HNA process, but were advised to do so. One Board regarded the process as an epidemiological exercise, and published an otherwise excellent set of data without community input. The provincial DHBs all consulted with and obtained data from the community and from community health providers, and found that they both provided valuable information. Other DHBs involved the community through focus groups, and even in working groups. Some DHBs expressed regrets that they had not involved the community in the HNA process, particularly regarding the "thinking and needs analysis design." Māori were generally included in key informant interviews to obtain the views of Iwi, Hapu, and urban Māori (hapu refers to sub-tribes of Māori). 


\section{Future work}

Most DHBs were considering, or had already commenced, collecting data on Disability Support Services at the time of interview (July-September 2002). The Wellington Clinical School was conducting a scoping exercise for DHBs on Disability Support Services HNAs. Other DHBs stated their intention to commence work in this area within the next 12 months. Some DHBs intended to develop Māori Health Plans, Primary Care Plans, and were considering youth and adolescent health and oral health projects in the future.

All DHBs saw HNAs as "living documents," providing a useful baseline to track the progress of health status and health need within the DHB population. All recognised that they were required by statute to repeat the HNA in three years' time.

\section{Costs of HNAs}

The cost of conducting HNAs varied from one DHB to another. The DHBs were asked to provide an all-inclusive estimate of cost, including staff time, external contractor costs, and printing and distribution. Their estimates, ranging from $\$ 30,000$ to $\$ 300,000$, are shown below.

Table 28: Cost of HNAs, by DHB, 2001.

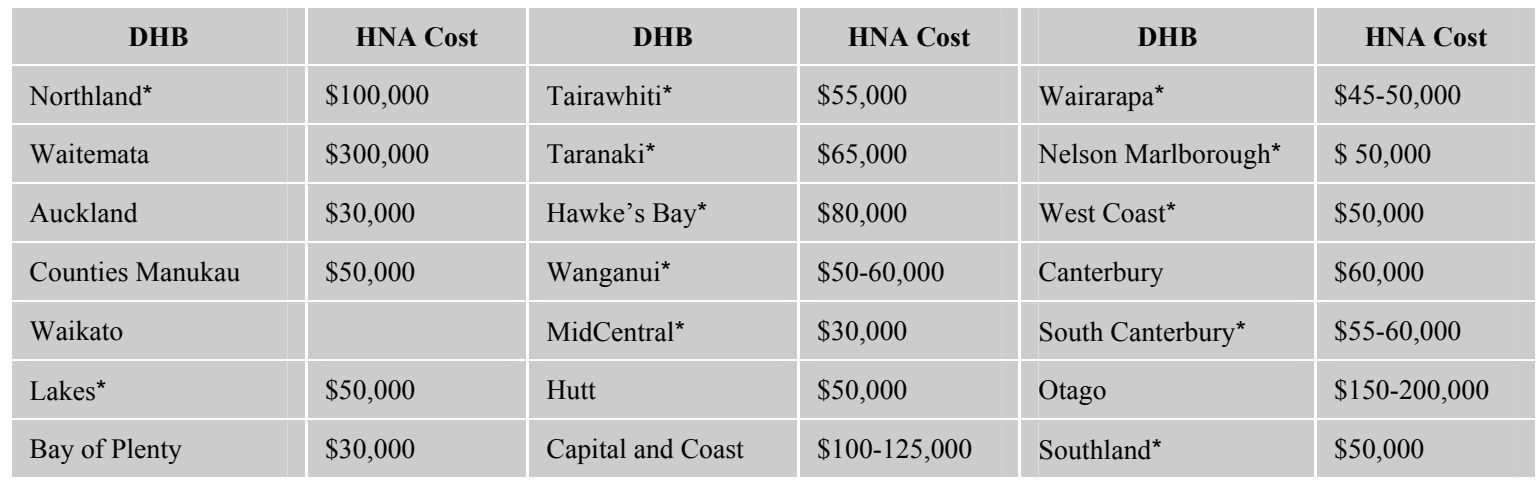

Source: DHB Planning and Funding Managers. HNA = Health needs assessment. *refers to those DHBs involved with the RHNAP. Note: The Waikato DHB did not participate in the first round of the Health Reforms 2001 research. It agreed to join the study in late 2003. 
The mean cost to DHBs of conducting HNAs was $\$ 75,000$. Three DHBs that were highly effective (see Impact Factors, p.229) averaged a cost of just $\$ 61,700$, showing that large expenditure was not necessary to achieve results. The DHBs that participated in the RHNAP had a mean HNA cost of $\$ 57,290$, whereas the larger DHBs that did not participate had a mean HNA cost of $\$ 80,930$. This may be a reflection of size. However, these mean costs are not statistically different using the 2-tailed students $t$-test $(p=0.24)$. Some of the excessive costs incurred by DHBs could have been avoided by using the centralised process, which also produced high-quality HNAs. All DHBs were required to work to the same minimum requirements set out by the Ministry. The graph below shows the HNA costs plotted as a function of the populations of DHBs, to test the relationship.

Figure 14: HNA costs as a function of DHB population

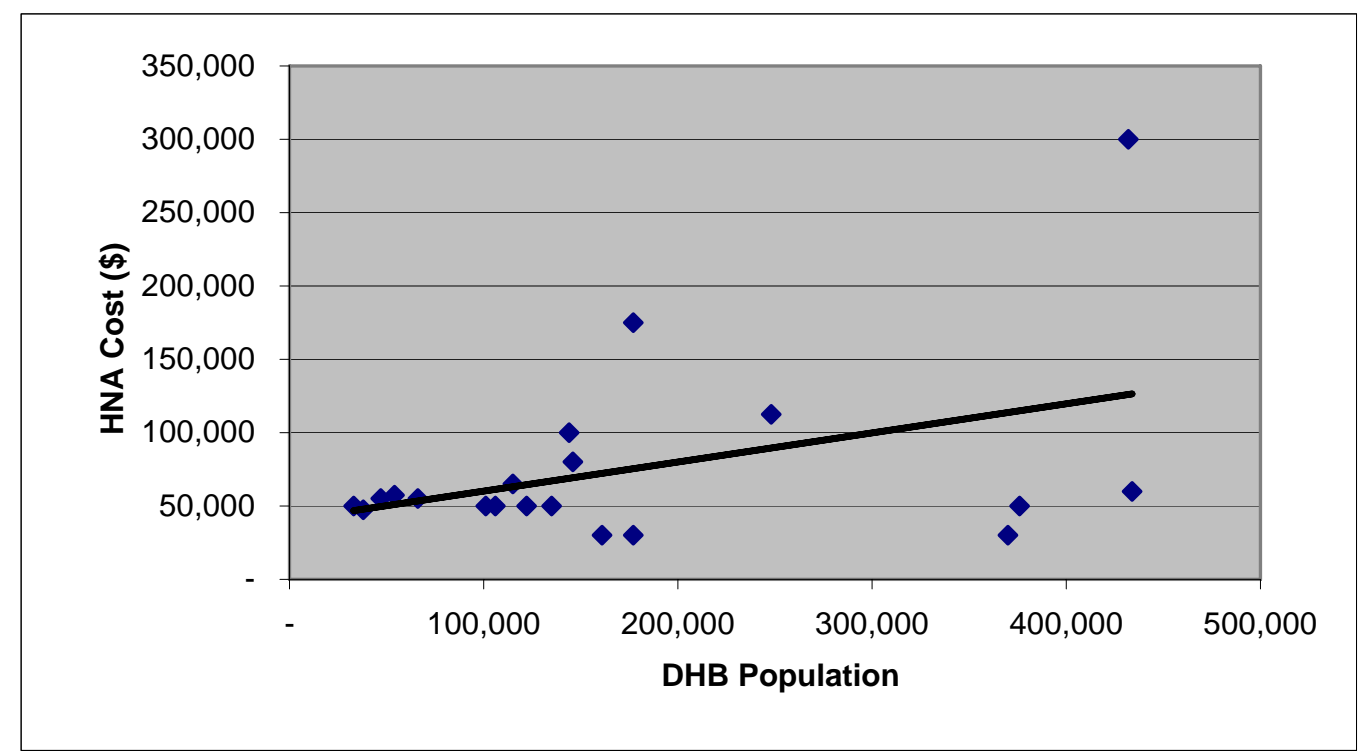

HNA=Health needs assessment; DHB = District Health Board; DHB population data refers to Census 2001 data.

This figure shows no statistically significant correlation at the $p=0.05$ level between the costs of conducting HNA (y) and DHB population size (x) (equation $\mathrm{y}=0.20 \mathrm{x}+$ $\left.40,285, \mathrm{p}=0.07, \mathrm{r}^{2}=0.17\right)$, although that relationship becomes close. 
The mean costs of conducting HNAs in DHBs accounted for $2.8 \%$ of Governance costs. $^{45}$ This compares with the $0.9-1.8 \%$ of UK health authorities' total management costs which were accounted for by needs assessment activity (Hensher and Fulop, 1999). It should be noted that needs assessment activity in the UK is different in that it is more focused on particular services. It is also difficult to be certain that management costs in different countries are in fact comparable.

\subsubsection{Prioritisation frameworks and prioritisation}

Planning and Funding Managers reported that DHBs took different approaches to prioritisation, particularly regarding involving the local community. It has been noted that some DHBs chose not to involve the community in the process, while others had significant community involvement. Government strategies, particularly the New Zealand Health Strategy with its the 13 priority objectives, were reported to be a powerful influence on all DHBs in the prioritisation process. Inevitably, DHBs found that they could not find the resources to address all 13 priority objectives, so they prioritised from the list, sometimes taking into account the HNA. Many had not completed their prioritisation framework when they undertook strategic planning, although some subsequently completed the framework and consulted on it alongside the DSP.

Most DHBs based their prioritisation frameworks on the Health Funding Authority (HFA) model. Some DHBs modified the framework so as to recognise the Government's 13 priority health objectives (for example, by adding 'consistent with the New Zealand Health Strategy and the New Zealand Disability Strategy.') But not all followed the HFA priorities, and instead used other criteria such as equity of access, equity of outcome, value for money, investing in the future, cultural appropriateness,

\footnotetext{
${ }^{45}$ Mean HNA cost is $\$ 75,000$, DHB Governance budget totals $\$ 56$ million ex GST, 21 DHBs, equates to $2.8 \%$.
} 
Treaty of Waitangi, and assurance of sustainable quality services. ${ }^{46}$ Others fitted in local priorities and objectives around innovation of service and equity of cost. Some developed variations using decision trees and flow charts for the DHB management to follow in prioritising. It was apparent that these would be applied only to allocation of new funding or funding that became available through management disinvestment in existing contracts.

A key finding from the interviews was that Funding Management Committees held significant power in a number of Boards. These Committees consisted of the Chief Executive Officer and a few key managers who made major purchasing decisions and therefore determined the final shape of DAPs prior to its ratification Board. The minutes of these Committees, if any were taken, were not available in the public domain. It is understood that Chairs of Boards were sometimes members of these Committees.

DHBs received Service Coverage Schedules from the Ministry of Health, setting out the Ministry's expectations regarding the levels of health services DHBs should provide. Some DHBs endeavoured to reduce deficits by reducing access to services (for example, one DHB sought to reduce access to fertility and sexual health services before the 2002 General Election, and was promptly told that this could not be done). Subsequently the Minister of Health advised DHBs that they were not to reduce health services without Ministerial approval. One DHB commented:

Government is pretty clear on what they want the money spent on, so it would be an interesting exercise if any District Health Board actually took the money and chose to spend it on something else because they undertook a prioritisation exercise and found something of higher value that they should be spending the money on instead. (DHB manager).

${ }^{46}$ DHB prioritisation frameworks have been analysed earlier and showed significant variability. Actual DHB priorities have also been analysed and are shown in Appendix 13, p.322. 
A number of other DHBs raised the issue of disinvestment, but quickly pointed out the difficulties of disinvesting in services because of Ministry of Health constraints. Another, commenting on the freedom of DHBs to make changes to services, noted, "a prioritisation framework is relevant where you have choices - and where there is not a choice, it is not relevant."

For most DHBs there was a sense that they were 'going through the motions' regarding the use of the prioritisation principles and frameworks, but that the real prioritisation was done at management and board level, by applying some or all of the 13 priority objectives of the NZHS.

Generally speaking, prioritisation was applied only to new funding, which formed but a small percentage of DHB budgets. Most DHBs stated that more than $99 \%$ of their budgets were pre-determined by existing contracts with health providers. New funding related generally to demographic changes, primary care, referred services and mental health budgets. Some DHBs were more creative and took a line-by-line approach to budget review, looking for non-performing contracts, and economies of scale by amalgamating contracts that were duplicating services. One said that it intended to be "extremely ruthless in renegotiation of contracts," to find funds to implement new initiatives in the DAP. Boards were given little time to develop prioritisation frameworks and DSPs, consult with communities and write DAPs. In summary, Boards did not have the time, commitment or resources to address reprioritisation.

DHBs were asked whether they were able to allocate resources according to local needs and values. They commented that devolved contracts had a long lead, generally because contracts had been signed for three-year terms, presenting a barrier to responding to health need. This meant that they could only respond to need at the margin, as contracts became due for review. Boards were generally not prepared to terminate contracts prematurely, unless there were performance issues. The environment for disinvestment was constrained by Government requirements, plans for reducing DHB deficits, and their limited freedom to reconfigure services. The problem was expressed this way: 
I think that the most effective way for the short or even the medium term is to ensure that any new funding that is made available, including any funding that is freed up, goes into priority areas, rather than be able to make huge strides as far as disinvestment occurs. (DHB manager)

A number of DHBs indicated that more money needed to go into primary care, and they were identifying possible sources within the provider arm (hospital services). For most, the reality of the Government requirement to eliminate DHB deficits, which were generated mainly by the provider arm, meant that a major focus remained on managing that part of the business. Some focused on the hope (and for some, expectation!) that the Population-Based Funding Formula (PBFF) would rescue them and that the situation would be much improved after $2004 .{ }^{47}$ Others were uncertain whether they would be better off with PBFF; and in fact some DHBs were to find that they were worse off as a result of falling populations. The effect of Inter District Flows (IDFs) had yet to be taken into account. ${ }^{48}$

DHBs noted that they had received price path adjusters of $1.7 \%$, mainly to account for demographic growth. ${ }^{49}$ Some boards received new funding for primary care; the other main source of new funding was that for mental health volumes, related to the implementation of the Mental Health Blueprint. Some also received additional funding for adolescent oral health. Almost all new funding was tagged, leaving DHBs with little scope for shifting resources within their budgets and therefore for reprioritisation.

47 The Population-Based Funding Formula (PBFF) is a formula for distributing health funding among DHBs. It takes account of gender, ethnicity, socio-economic deprivation and rurality. Some boards receive 'one-line adjusters' for the provision of tertiary services. The funding received by DHBs changes with the population and demographic mix.

${ }^{48}$ IDFs take account of the costs of delivering health services by a DHB for patients from another DHB. An IDF occurs when the DHB that has provided the service is paid by the DHB where the patient normally resides.

${ }^{49}$ Price path adjuster is the Ministry of Health's term for additional funding lines (price paths) provided in addition to existing funding, for example to accommodate demographic growth. 
There was little evidence that DHBs applied health economic principles to prioritisation. Only one board had undertaken any form of cost-utility analysis (CUA) - Hutt DHB considered quality of life and marginal cost. ${ }^{50}$

DHBs have an interest in PHARMAC, in that it manages the DHBs' pharmaceutical budget on behalf of DHBs. PHARMAC uses a prioritisation process that includes health economic analysis, by means of CUA. So in that limited sense, DHBs indirectly use CUA to prioritise pharmaceutical purchasing.

Boards were clearly experiencing difficulties reallocating existing funding within their budgets. One DHB manager reported that they were able to realign budgeted volumes to actual volumes and thereby reduce medical case weights ${ }^{51}$ because admission durations were falling, freeing some funding for the 02/03 budget. All DHBs were finding it difficult to eliminate DHB budget deficits within the three-year period required by the Ministry of Health. (This observation preceded the announcement in December 2002 that DHBs would be given additional funding to address deficits, over a three-year period. In the event, the extra funding did not allow DHBs to resolve deficits - planned deficits totalled $\$ 185.9 \mathrm{M}$ for the 2002/03 year.)

\subsubsection{Key influences on DSPs}

The factors quoted by DHBs as key influences on DSPs were legislation, Ministry guidelines, historical factors, HNAs, community preferences, and the financial constraints of the funding environment, including DHB deficits. Government strategies and the 13 priority objectives strongly determined the direction of DSPs, all of which had to be signed off by the Ministry of Health. DHBs received strong messages that the

\footnotetext{
${ }^{50}$ Cost Utility Analysis (CUA) compares the costs and effectiveness of interventions in terms of their usefulness in producing life years gained (PLYG), the quality of these life years (QALYs), or disability free years (DALYs).

51 'Medical case weights' are weightings assigned at discharge diagnosis, based on length of stay averaged for similar admissions. Medical case weights fall as the duration of inpatient stay reduces.
} 
Government's priority objectives had to be reflected in DSPs, and it appears that this was an overwhelming influence on decision-making. DHBs could select priority areas from those objectives to reflect local priorities. Among other factors quoted as important was the need to break even within three years, to develop primary care, and to improve access for rural communities. Some DHBs reported that community consultation had little influence on their decision-making, whereas consultation with Māori resulted in change for most boards. Pressure from stakeholders (other than Board Members) was uncommon and reported to be manageable. One DHB reported that the HNA process “didn't have a huge impact on our planning process."

\section{Community consultation on District Strategic Plans}

Managers reported that Boards used a wide variety of means to consult with their communities. Examples included public meetings, hui and marae visits, fono, websites, written and oral submissions, (sometimes using feedback forms), letter-box drops, access radio, advertisements in newspapers, presentations to local and regional councils, and distributing drafts to key community groups for feedback. One DHB held an 'invitation week' where members of the public and providers could come to the DHB to give their views to HNA managers.

Generally DHBs had an external note-taker or a staff member taking notes during community consultation at public meetings. Feedback was analysed, submissions summarised in tables, and where it was considered appropriate feedback was incorporated into DSPs. In some cases, feedback included concerns about transport, housing, water and food, and these issues were referred to appropriate agencies. DHBs commented that feedback generally confirmed the existing understandings of DHB managers. In several instances significant health service needs were identified, including access for deaf people and refugees, and these were incorporated into plans.

DHBs noted that low socio-economic groups generally did not have a direct impact on the HNA or the DSP itself. They commented that their input was difficult to obtain as members of such groups generally had no telephone, no newspaper, no transport, and 
rarely attended public meetings. However it was thought that input as to their needs was derived indirectly, from data rather than consultation. DHBs also felt that they obtained a perspective on the needs of this group through community contacts, but considered that this was not a substitute for engaging directly.

\subsubsection{Linkages between DAPs and budgets}

The Ministry of Health directed that for each board the DAP was to be based on the DSP. A number of DHBs identified a strong connection between their DAPs and DSPs, but others noted weak connections. Generally DHBs started with their budgets then aligned DAPs to ensure consistency, taking into account new plans. As one DHB manager put it, "I think that the budget is driving the DAP". A number of DHBs stated that in the first year DAPs bore less relationship to DSPs than they considered desirable, or likely in subsequent years. DHBs were required to show a three-year pathway for eliminating their deficits.

DHBs noted that any greater separation of HNA and planning was likely to lead to loss of key recommendations from the needs assessments. Some felt that HNAs almost required an advocate to ensure their 'voice' was heard in the process. One interviewee put it like this:

I believe that the HNA is our most valuable document, and it should be the baseline reference for anything else that we do. I think that the danger that we run is that in running from HNA to DSP to DAP to budgets and purchasing we lose the key components within the HNA. I think that we must talk up the HNA more so that the other documents that tend to water down some of the key statistics within the HNA...to my mind we are losing some of the key components of the HNA. And they need to stay connected.

Another small DHB said that there was so much health need that required addressing, that the health need assessment did not help particularly:

I think that if you are in a DHB that had some things that were glaringly obvious, it would be quite well joined-up. If you were in a DHB like us where there are so many things that need to be fixed up, it assists the planning process but it doesn't provide the answers...It does work out that the priority areas we get fit in with the priority areas of Government, so I mean all our ducks are lining up in a row. 
Another DHB commented regarding prioritisation and the DSP: "I don't personally feel they are joined-up. I think that prioritisation is a process sitting outside the strategic plan document itself, but didn't influence it." DHBs faced a number of challenges in connecting HNA, prioritisation, DSP, DAP and the budget for the 02/03 year.

\subsubsection{Connections between health needs assessments and purchasing}

\section{Introduction}

To ensure that DHBs met the health needs of their populations by funding and purchasing health services, they were required to connect HNA and prioritisation to the DHB Plans during the planning process. Planning and Funding Managers were asked how well the various components of the pathway were 'joined-up'. They gave enough information to conduct an analysis of the strength of that connection. It was also possible to triangulate this information with information from documents including HNAs, DSPs, DAPs and prioritisation.

\section{Analysis of connections from HNA to purchasing}

The method for analysing Planning and Funding manager interviews for the strength of connection between HNA, prioritisation, DSPs, and DAPs has been described previously (see Chapter 5.3.3, p.138). Each step in the process from HNA to purchasing was assessed by Planning and Funding Managers, and the researcher allocated a score, with a maximum of 3 , on the basis of managers' assessments. This gave rise to (total) Connection Scores that ranged from 8 to 14, with a possible maximum of 15 points. 
Table 29: Connection Scores for the steps from HNA to Purchasing (assessed using data from the DHB Planning and Funding Managers' interviews).

\begin{tabular}{|c|c|c|c|c|c|c|}
\hline DHB & $\begin{array}{c}\text { HNA \& } \\
\text { Prioritisation }\end{array}$ & $\begin{array}{c}\text { Prioritisation } \\
\text { \& DSP }\end{array}$ & DSP \& DAP & DAP \& Budget & $\begin{array}{l}\text { Budget \& } \\
\text { Purchasing }\end{array}$ & $\begin{array}{c}\text { Connection } \\
\text { Score }\end{array}$ \\
\hline Northland & 3 & 2 & 3 & 2 & 3 & 13 \\
\hline Waitemata & 3 & 3 & 2 & 2 & 3 & 13 \\
\hline Auckland & 1 & 2 & 2 & 3 & 2 & 10 \\
\hline Lakes & 2 & 2 & 3 & 3 & 2 & 12 \\
\hline Bay of Plenty & 3 & 3 & 2 & 2 & 3 & 13 \\
\hline Tairawhiti & 1 & 3 & 2 & 3 & 3 & 12 \\
\hline Whanganui & 3 & 2 & 2 & 2 & 3 & 12 \\
\hline MidCentral & 1 & 3 & 2 & 2 & 3 & 11 \\
\hline Hutt & 3 & 3 & 2 & 3 & 3 & 14 \\
\hline Capital and Coast & 1 & 3 & 0 & 2 & 3 & 9 \\
\hline Wairarapa & 3 & 3 & 3 & 2 & 3 & 14 \\
\hline Nelson Marlborough & 0 & 3 & 3 & 1 & 3 & 10 \\
\hline Southland & 3 & 3 & 1.5 & 3 & 3 & 13.5 \\
\hline Mean & 1.8 & 2.4 & 2.12 & 2.25 & 2.75 & \\
\hline
\end{tabular}

(3=strong, $2=$ moderate, $1=$ =weak, $0=$ none. HNA=HNA; DSP= District Strategic Plan; DAP= District Annual Plan.)

No significant difference was found between the mean Connection Scores of the 12 DHBs participating in the RHNAP process and of those who conducted their own HNA process: mean score $11.71(\mathrm{SD}=1.74,95 \% \mathrm{CI}=10.73-12.69)$; and for the 8 non-RHNAP DHBs, mean score $10.75(\mathrm{SD}=2.11,95 \% \mathrm{CI}=9.29-12.21)$.

Table 30 shows that for all DHBs, the strongest overall correlation (mean score 2.75) is between budget and purchasing. The strength of connection between HNA and prioritisation yields the lowest mean score (1.8). This reflects how difficult DHBs had found prioritising services on the basis of health need. It appears that the difficulty arose 
because Ministry directives prevented DHBs from disinvesting in services, and that boards considered prioritisation therefore largely irrelevant, except as a means of allocating 'new money'.

The size of the DHB appears to bear no relationship to the success of the process, as medium-sized and small DHBs feature among the more successful, and large and small DHBs among those the less successful (see Table 30). It is important to remember that Connection Scores are derived from self-reporting by DHB Planning and Funding Managers, and that they may have either overestimated or underestimated the degree to which the components are connected.

\subsection{Impact Factor - a measure of the impact of health needs assessments on planning and purchasing}

To determine the impact of HNA on planning and purchasing, an Impact Factor was calculated for each DHB, based on the analysis of documents from the HNAs, DSPs and DAPs. The Methods chapter outlines the method for deriving Impact Factors (see Chapter 5.3.3, p.138).

Impact Factors and Connection Scores were then compared to triangulate data. Quality scores are also recorded for comparison purposes (see Table 25). Raw data are listed in Table 30 below and are plotted in Figure 15. 
Table 30: Impact Factors, Connection Scores, HNA Quality Scores and DHB deficits as a percentage of budget.

\begin{tabular}{|c|c|c|c|c|c|c|}
\hline DHB & $\begin{array}{c}\text { Impact } \\
\text { Factor } \\
(\max =5)\end{array}$ & $\begin{array}{c}\text { Connection } \\
\text { Score } \\
(\max =15)\end{array}$ & $\begin{array}{c}\text { HNA } \\
\text { Quality } \\
\text { Score }\end{array}$ & $\begin{array}{c}\text { Planned } \\
\text { Deficit 02/03 }\end{array}$ & $\begin{array}{c}\text { Budget } \\
02 / 03\end{array}$ & $\begin{array}{c}\text { \% Deficit } \\
\text { /Budget }\end{array}$ \\
\hline Hutt & 5 & 14 & 39 & $\$ 0.1 \mathrm{~m}$ & $\$ 172.8 \mathrm{~m}$ & $0 \%$ \\
\hline Hawke's Bay* & 4.07 & 14 & 44 & $\$ 6.3 \mathrm{~m}$ & $\$ 124.5 \mathrm{~m}$ & $5.1 \%$ \\
\hline Whanganui* & 3.77 & 12 & 44 & $\$ 4.5 \mathrm{~m}$ & $\$ 93.6 \mathrm{~m}$ & $4.8 \%$ \\
\hline Canterbury & 3.5 & 8 & 18 & $\$ 11.5 \mathrm{~m}$ & $\$ 700.3 \mathrm{~m}$ & $1.6 \%$ \\
\hline $\begin{array}{l}\text { Nelson } \\
\text { Marlborough* }\end{array}$ & 3.5 & 10 & 44 & $\$ 1.3 \mathrm{~m}$ & $\$ 164.0 \mathrm{~m}$ & $0.8 \%$ \\
\hline Lakes* & 3.44 & 12 & 44 & $\$ 4.3 \mathrm{~m}$ & $\$ 115.4 \mathrm{~m}$ & $3.7 \%$ \\
\hline Wairarapa $^{*}$ & 3.44 & 14 & 44 & $\$ 0.4 \mathrm{~m}$ & $\$ 48.8 \mathrm{~m}$ & $0.8 \%$ \\
\hline Auckland & 3.32 & 10 & 39 & $\$ 61 \mathrm{~m}$ & $\$ 873.7 \mathrm{~m}$ & $7.0 \%$ \\
\hline Southland* & 3.28 & 13.5 & 44 & $\$ 5.3 \mathrm{~m}$ & $\$ 109.4 \mathrm{~m}$ & $4.8 \%$ \\
\hline Otago & 3.05 & 9 & 29 & $\$ 10.8 \mathrm{~m}$ & $\$ 317.1 \mathrm{~m}$ & $3.4 \%$ \\
\hline Tairawhiti* & 3.04 & 12 & 44 & $\$ 0.8 \mathrm{~m}$ & $\$ 65.8 \mathrm{~m}$ & $1.2 \%$ \\
\hline $\begin{array}{l}\text { Counties } \\
\text { Manukau }\end{array}$ & 3.0 & 10 & 26 & $\$ 21.7 \mathrm{~m}$ & $\$ 360.7 \mathrm{~m}$ & $9.3 \%$ \\
\hline Northland* & 2.86 & 13 & 44 & $\$ 3.5 \mathrm{~m}$ & $\$ 190.0 \mathrm{~m}$ & $1.8 \%$ \\
\hline $\begin{array}{l}\text { Capital and } \\
\text { Coast }\end{array}$ & 2.8 & 9 & 17 & $\$ 0.7 \mathrm{~m}$ & $\$ 414.1 \mathrm{~m}$ & $0.2 \%$ \\
\hline Taranaki* $^{*}$ & 2.75 & 10 & 44 & $\$ 4.2 \mathrm{~m}$ & $\$ 125.3 \mathrm{~m}$ & $3.4 \%$ \\
\hline West Coast* & 2.75 & 8 & 44 & $\$ 2.5 \mathrm{~m}$ & $\$ 57.0 \mathrm{~m}$ & $4.4 \%$ \\
\hline Waitemata & 2.67 & 13 & 39 & $\$ 12.0 \mathrm{~m}$ & $\$ 425.1 \mathrm{~m}$ & $2.8 \%$ \\
\hline MidCentral $^{*}$ & 2.3 & 11 & 44 & $\$ 9.3 \mathrm{~m}$ & $\$ 243.0 \mathrm{~m}$ & $3.8 \%$ \\
\hline Bay of Plenty & 2.04 & 13 & 20 & $\$ 6.5 \mathrm{~m}$ & $\$ 233.6 \mathrm{~m}$ & $2.8 \%$ \\
\hline $\begin{array}{l}\text { South } \\
\text { Canterbury* }\end{array}$ & 1.41 & 11 & 44 & $\$ 0.9 \mathrm{~m}$ & $\$ 70.4 \mathrm{~m}$ & $1.3 \%$ \\
\hline
\end{tabular}

Note: DHBs in the RHNAP process are marked with an asterisk. 
Figure 15: Relationship between Connection Scores and Impact Factors for DHB HNAs

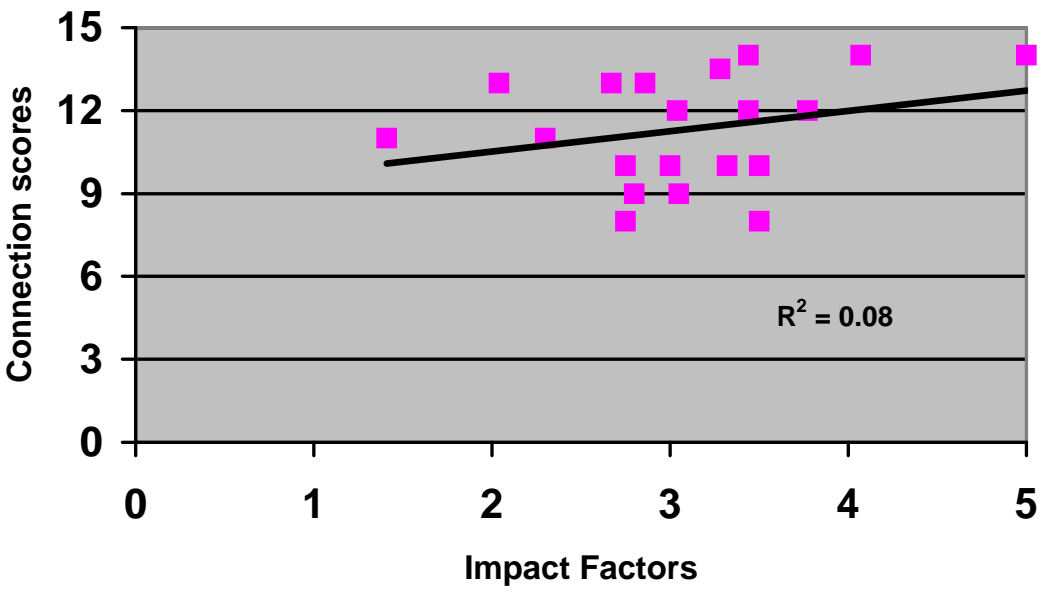

Figure 15 shows that there is no significant relationship at the $p=0.05$ level between Connection Scores $(y)$ and Impact Factors $(x)$ (equation $y=0.73 x+9.05, p=0.24, r^{2}=$ 0.08). Because Impact Factors are more objective since they were derived by document analysis, they have been preferred for inter-DHB comparisons rather than Connection Scores, which are based on the opinions of Planning and Funding Managers.

Analysis using a Pearson's (product moment) correlation also demonstrated no statistically significant association at the 0.05 level between any of the variables (HNAs, Quality Scores, Connection Scores and Impact Factors), see Table 31 below.

Table 31: Pearson Correlations for Impact Factors, Connection Scores, HNA Quality and DHB Deficits.

\begin{tabular}{|l|l|c|c|c|c|}
\hline & & IMPACT F & CONNECTION & HNA QUALITY & DHB DEFICIT \\
\hline IMPACT F & Pearson Correlation & 1 & .227 & .122 & -.021 \\
\hline & Sig. (2-tailed) &. & .237 & .608 & .930 \\
\hline & N & 20 & 20 & 20 & 20 \\
\hline & Pearson Correlation & .277 & 1 & .410 & -.134 \\
\hline & Sig. (2-tailed) & .237 &. & .073 & .575 \\
\hline & N & 20 & 20 & 20 & 20 \\
\hline & Pearson Correlation & .122 & .410 & 1 & .028 \\
\hline & Sig. (2-tailed) & .608 & .073 &. & .908 \\
\hline & N & 20 & 20 & 20 & 20 \\
\hline & Pearson Correlation & -.021 & -.134 & .028 & 1 \\
\hline & Sig. (2-tailed) & .930 & .575 & .908 &. \\
\hline
\end{tabular}


Planning and Funding Managers maintained that DHBs had difficulty incorporating HNAs into the planning process because of DHB deficits and because it is difficult to make disinvestments or change funding allocations. If this were so, it would be expected that DHBs with large deficits would find that HNAs had little impact on planning. To test this, Connection Scores and Impact Factors were plotted against the size of DHB deficits, expressed as a percentage of the total DHB budget for the 2002/03 year.

Figure 16: Connection Scores and Impact Factors as a function of DHB deficits \% of budget, Yr 2002/03

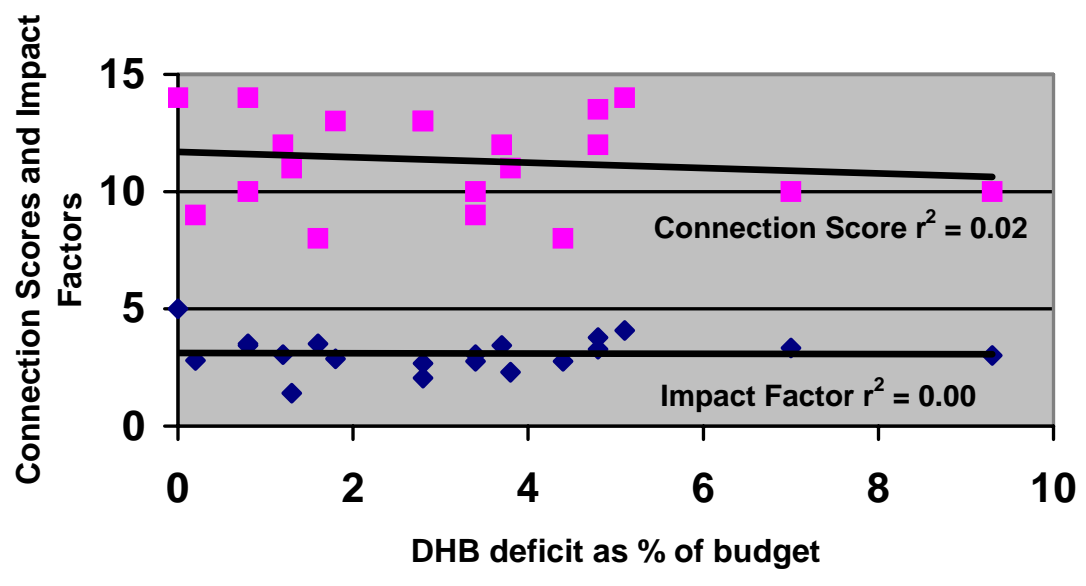

Figure 16 shows no statistically significant relationship at the $p=0.05$ level between the Connection Score (y) and the size of the DHB deficit as a percentage of total DHB budget $(\mathrm{x})$ (equation $\mathrm{y}=-0.11 \mathrm{x}+11.68, \mathrm{p}=0.57, \mathrm{r}^{2}=0.02$ ). Similarly there is no statistically significant relationship at the $\mathrm{p}=0.05$ level between the Impact Factor (y) and the size of the DHB deficit as a percentage of total DHB budget (x) (equation $\mathrm{y}=$ $\left.-0.007 \mathrm{x}+3.12, \mathrm{p}=0.93, \mathrm{r}^{2}=0.00\right)$.

The data shows that there was no statistically significant relationship at the $p=0.05$ level between either Connection Scores, or Impact Factors, and DHB deficits expressed as a percentage of the total DHB budget for the 2002/03 year, and therefore does not 
support Planning and Funding Managers assertions regarding the positive influence of DHB deficits on decision-making.

The next question is whether or not the quality of HNAs bears any relationship to their impact on priorities, planning and purchasing. To test that relationship, Impact Factors are plotted against Quality Scores.

Figure 17: Relationship between Impact Factors and HNA quality scores

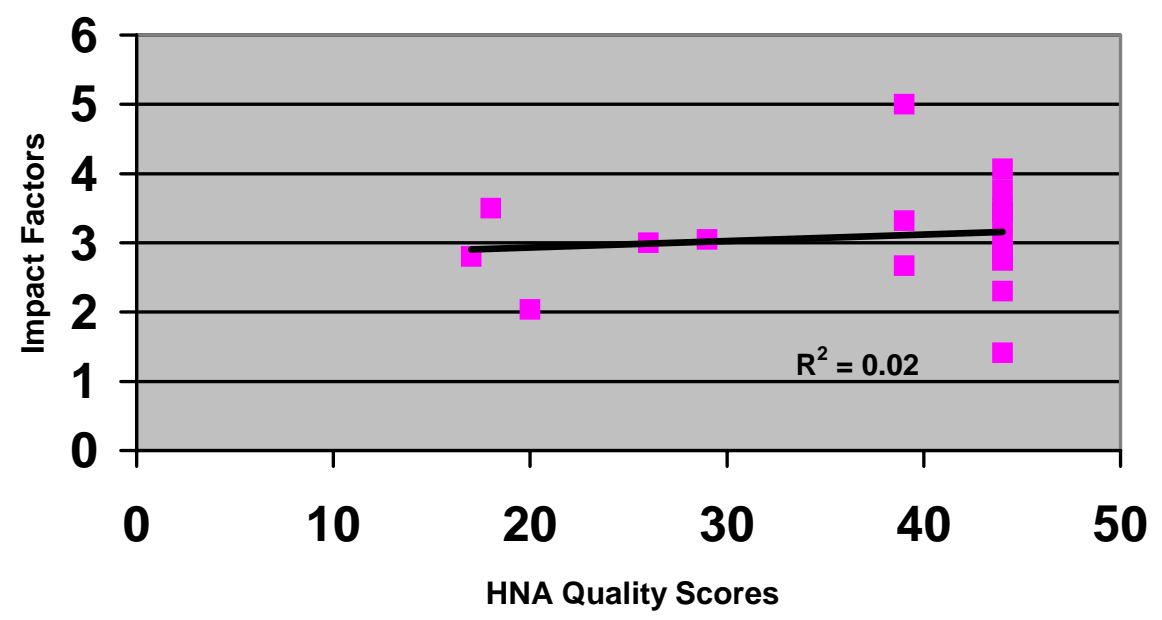

The analysis indicates that there is no statistically significant relationship at the $p=0.05$ level between the Impact Factors (y) and the Quality Score of HNAs (x) (equation y = $\left.0.009 x+2.74, p=0.61, r^{2}=0.02\right)$. Furthermore, no statistically significant difference was found between the Impact Factors of those in the RHNAP process (which had HNA Quality Scores of 44: mean $3.05(\mathrm{SD}=0.71,95 \% \mathrm{CI}=2.65-3.45)$ and those which were not: mean 3.17 ( $\mathrm{SD}=0.86,95 \% \mathrm{CI}=2.57-3.77)$. Similarly, no relationship was found between HNA Quality Scores and Connection Scores. 


\subsection{Case Studies}

It is evident that the HNAs of some DHBs had a significantly higher impact on the planning process than others. As has already been demonstrated, impact bears no relationship to the quality of HNAs, or to deficits, and so the question arises of why some DHBs do better than others. Such factors as timing, the experience of planning managers, the internal dynamics of the organisation, and the planning process itself, may affect the outcomes of planning (these factors relate to the links of HNA to a discernable policy/decision process). This hypothesis is now explored. Rather than examine the planning processes of every DHB, three case study DHBs with highimpact HNAs have been chosen alongside two with low-impact HNAs, to see what factors were operating. The DHBs whose HNAs had the greatest impact were Hutt Valley, Hawkes Bay, and Whanganui. The case study DHBs chosen from among those with the least impact of HNA were West Coast (sixth lowest) and South Canterbury.

It should be noted that the case study DHBs chosen for this research are not the same as those chosen by the Health Reforms 2001 project. The latter case studies were chosen to be representative of size, urban and rural, and geographical location, while for this research they are representative of high and low Impact Factors.

Interview transcripts with Planning and Funding Managers from the case study DHB were read and re-read, searching for evidence in the various steps of the process from HNA to budgeting. Table 32 shows the HNA, prioritisation and consultation activities and approaches undertaken by case study DHBs. Under each of these headings, activities and sub-activities were recorded to form a picture of the various approaches taken by the DHBs.

It is concluded that DHBs that participated in the RHNAP produced better-quality HNAs than did other DHBs, but there were no significant differences between the two groups in effectiveness of the HNAs. 
Table 32: Activities undertaken by Case study DHBs in HNA, prioritisation and consultation.

\begin{tabular}{|c|c|c|c|c|c|c|c|c|c|c|}
\hline \multicolumn{2}{|c|}{ DHB } & \multicolumn{6}{|c|}{ HNA } & \multicolumn{3}{|c|}{ Planning } \\
\hline \multirow{2}{*}{ DHB } & \multirow{2}{*}{$\begin{array}{l}\text { Impact } \\
\text { Factor }\end{array}$} & \multirow{2}{*}{ RHNAP } & \multirow{2}{*}{$\begin{array}{l}\text { Local } \\
\text { Advisory } \\
\text { Group }\end{array}$} & \multirow{2}{*}{$\begin{array}{c}\text { Commun } \\
\text { ity } \\
\text { Surveys }\end{array}$} & \multirow{2}{*}{$\begin{array}{c}\text { Key } \\
\text { Informant } \\
\text { Interviews }\end{array}$} & \multicolumn{2}{|c|}{ Involvement } & \multirow{2}{*}{ Model } & \multirow{2}{*}{$\begin{array}{l}\text { Planning } \\
\text { Groups }\end{array}$} & \multirow{2}{*}{$\begin{array}{l}\text { Consulta } \\
\text { ion }\end{array}$} \\
\hline & & & & & & Community & Māori & & & \\
\hline Hutt Valley & 5 & & & & & $\checkmark$ & $\checkmark$ & MSM & 7 & $\checkmark$ \\
\hline $\begin{array}{c}\text { Hawke's } \\
\text { Bay }\end{array}$ & 4.07 & $\checkmark$ & $\checkmark$ & $\checkmark$ & $\checkmark$ & $\checkmark$ & $\checkmark$ & MSM & 11 & $\checkmark$ \\
\hline Whanganui & 3.77 & $\checkmark$ & $\checkmark$ & & $\checkmark$ & $\checkmark$ & $\checkmark$ & CRPM & 1 & $\checkmark$ \\
\hline West Coast & 2.75 & $\checkmark$ & $\checkmark$ & $\checkmark$ & $\checkmark$ & $\checkmark$ & $\checkmark$ & CRPM & 1 & $\checkmark$ \\
\hline $\begin{array}{c}\text { South } \\
\text { Canterbury }\end{array}$ & 1.41 & $\checkmark$ & $\checkmark$ & & $\checkmark$ & $\checkmark$ & $\checkmark$ & CRPM & 1 & $\checkmark$ \\
\hline
\end{tabular}

RHNAP = Regional HNA Project; $\mathrm{MSM}=$ mixed scanning model; $\mathrm{CRPM}=$ comprehensive rational planning model; Planning Groups = Service Planning Groups focused on priority service areas with representation from community, Māori, and DHB staff. Note: Hutt Valley, Hawkes Bay, and Wairarapa DHBs were the only DHBs to use a mixed scanning approach.

\subsubsection{Case study A (Hutt Valley DHB)}

\section{Mixed scanning model}

Using an approach that was implicitly based on a bounded rationality planning/policy model, ${ }^{52}$ management identified priority service areas for in-depth planning, based on key objectives for priority attention set out in the New Zealand Health Strategy. This was a crucial step. HNA data were then collected and provided to seven service planning groups in March 2001. ${ }^{53}$

52 The term bounded rationality is used to designate rational choice that takes into account the cognitive limitations of both knowledge and cognitive capacity. Planning using bounded rationality implies that rational individuals make decisions which appear to them to be the best in the circumstances, based on their knowledge, however limited or expansive it is. The downside is that a better option may be passed over because of lack of information.

${ }^{53}$ A service planning group or service development group refers to a group of managers, providers, Māori providers, Iwis and community representatives given the task of planning a defined service or programme. 
Table 33: Hutt Valley DHB service planning groups, and specific service plans (November 2001)

\begin{tabular}{|c|c|}
\hline Service Planning Groups (March 2001) & Specific Service Plans (November 2001) \\
\hline Healthy Communities & - Healthy Communities \\
\hline Child \& Family & $\begin{array}{l}\text { Maternity } \\
\text { - } \\
\text { - Oral Health }\end{array}$ \\
\hline Youth & - Youth \\
\hline Chronic Diseases & $\begin{array}{l}\text { - Cardiovascular } \\
\text { - } \text { Respiratory } \\
\text { - } \text { Cabetes } \\
\text { - Surgical }\end{array}$ \\
\hline Primary Care & - Primary Care \\
\hline Mental Health & - Mental Health \\
\hline Disability Support & - Disability Support \\
\hline
\end{tabular}

Source: (Hutt Valley District Health Board, 2002, p.5)

The key tasks of the service planning groups for this DHB included:

- Reviewing demographic data relating to the service and population.

- Reviewing service access and use and identifying service gaps.

- Assisting in identifying the health and disability support needs of the Hutt Valley community, by:

- Learning about broad health and disability support needs and priorities through community consultation.

- Highlighting the areas of unmet need and ascertaining whether effective strategic responses exist.

- Identifying where issues relate to several sectors and developing intersectoral strategies to address such gaps, including the identification of the organisations that need to be involved and how this will occur.

- Identifying where issues relate to several sectors and developing intersectoral strategies to address such gaps, including identification of the organisations that need to be involved and how this will occur.

- Identifying programmes that are specific to Màori and Pacific people.

- Developing a comprehensive understanding of health services currently provided.

- Identifying opportunities to improve health outcomes in the local population.

- Developing service plans for the areas falling within the scope of each service planning group. (Hutt Valley District Health Board, 2002, p.6) 
Each service planning group generally included several representatives from the Māori and Pacific communities, senior medical staff, a GP, community representatives and where possible providers from non-governmental organisations. ${ }^{54}$ Community members of the service planning groups were selected by an open community process, where anyone could be nominated. One Māori representative was generally from an Iwi, and the other from a local Māori provider. Over 120 people in seven groups were involved in the process. The DHB worked with each group over four months to conduct HNAs and develop service plans for seven key service areas for the next five years, focusing on priorities and key issues for that area. Planning and Funding staff did most of the work, collecting demographic data and current spending information, and provider stocktake information. The exercise was described as a logistical "nightmare" with large volumes of minutes, background papers, and data gathered for analysis. Each meeting had a facilitator and backup assistant, a preset agenda, and a process for identifying issues, gathering information, interpreting it and obtaining priorities.

Each service planning group prepared a draft service plan (including needs analysis) for the Hutt Valley DHB Board to review. This was done in November 2001. The final outcomes of this assessment were reflected in high-level service planning summaries (each two to three pages long) in the draft service plan. The summaries included a strategy tree for each service plan and prioritised the strategies. ${ }^{55}$

\footnotetext{
${ }^{54}$ Non-governmental organisations refer to private organisations such as mental health service providers, rest home trusts, and Primary Health Organisations.

${ }^{55}$ A strategy tree was a diagram that set out a structured approach to strategy determination. The strategy tree components were the broad goals in each planning area, linked to key national expectations; the objectives to pursue to meet the goals; the strategies (or actions) that were to be followed to meet the objectives; and the performance targets and indicators to measure whether the selected strategies had been effective in addressing the goals. Hutt Valley District Health Board (2002) Towards a Healthier Community: The Five Year District Strategic Plan 1 July 2002 to 30 June 2007. Hutt Valley District Health Board, Hutt Valley. Further details of the actual service plans are available at www.huttvalleydhb.org.nz
} 
The result was 13 needs assessments and service plans, which were consulted on as part of the district strategic planning process. Although there was not one to one correspondence, the $13 \mathrm{HNA}$ and service plans covered all 13 Government priority objectives. In addition, the service plans included performance indicators so that the DHB could establish whether it was making a difference to performance, and could also monitor progress.

DHB staff commented that if they were doing the exercise again, they would use the same model, but run the group processes using more of a workshop approach involving fewer meetings, and less checking of drafts by the groups.

\section{Prioritisation}

Service planning groups identified 41 areas for future investment. These were then prioritised using the decision-making principles determined by the Board:

- Effectiveness (the likelihood and the importance of the benefits that will come from the initiative).

- Equity (whether the initiative is aimed at those most disadvantaged currently so as to try to give all people equal chances of long, healthy lives).

- Acceptability (whether the community will accept the initiative).

- Consistency with the New Zealand Health Strategy (whether the initiative supports the Government's top priorities for health).

- Cost (how much per person the initiative will cost, and what savings it may produce by reducing illness and preventing the need for treatment). (Summarised from(Hutt Valley District Health Board, 2002, p.10))

The proposed areas of spending were scored according to these principles, and the resulting prioritised list of initiatives included in the Draft Five-year District Strategic Plan, which then went to public consultation.

The application of cost-utility analysis to all classes of DHB spending was considered, but because there was limited analytical capacity within the DHB and also a lack of clear information in some cases, it was decided that it would be very difficult to apply that form of analysis across different programmes. It was therefore applied only to 
selected interventions for specific classes of individuals. The process adopted considered cost offsets, benefits to individuals, quality of life, length of life, marginal cost, who benefits, equity issues, and Māori health issues. Two experts in cost-utility analysis and prioritisation independently critiqued the Hutt Valley DHB (HVDHB) prioritisation methodology in February 2002 (Hefford, 2002). In general, both reviewers supported the approach and methodology and thought that HVDHB had developed a robust and pragmatic approach to prioritisation given the available resources. Most of the changes they suggested were concerned with improving the clarity of the methodology.

Proposed initiatives were presented initially to the Community and Public Health Advisory Committee and subsequently to the Board, which reviewed the recommendations, and decided what weighting should be given to the various prioritisation principles. Māori participated through consultation with community, including hui, and the contribution of three Māori board members.

After considering service plans, and prioritising, the DHB produced a list of new initiatives deemed to be of greatest benefit to the community as a whole - "the most effective way of spending any new funding." The DHB then invited public comment on whether the prioritisation principles it applied seemed valid, and whether the ranking of 20 (out of 41) new initiatives for spending \$6 million of new funding (should it be made available by Government) was correct (Hutt Valley District Health Board, 2002). It was intended that the Board review the prioritisation methodology in March 2003.

At interview, the DHB's Planning and Funding Manager noted that freedom to allocate resources on the basis of local needs and values was constrained by the Crown Funding Agreement, the DAP, and Government requirements for service coverage. The DHB observed that the Government had two sets of priorities: those set out in the New Zealand Health Strategy; and those that are manifest in the allocation of funding. These priorities, plus historical spending patterns reflected in existing contracts, determined most of the allocation of budgets under the DAP. 


\section{Consultation}

Consultation with the community in the DSP process involved the distribution of Towards a Healthier Community: A Draft 5-Year Strategic Plan for Consultation (12pp) to all homes in the district, together with freepost feedback sheets; sending full DSPs to main stakeholders and providers; presentations to key groups; and public meetings. The Planning and Funding Manager made the following comment regarding the usefulness of the HNA:

I think one of the reasons why it was useful is that we did it, not as an isolated exercise, but as an integral part of the planning process. So it was built into the service planning right from the start, even to the point were we would have a meeting with a service planning group and they would say that 'this was useful information but it is all very well what we need to know is this and this.' We would go away and find out those things and bring them back to the group, so it was a live interactive process, rather than go away and do a needs assessment, write an epic, and distribute it to people. (manager)

In the view of the DHB, the process allowed Māori and low socio-economic groups (including lobbyists acting on their behalf, and the Union Health Clinics) to have an influence. The consultation process was therefore regarded as broad reaching.

\section{Comment}

HVDHB was the only DHB to achieve the highest possible Impact Factor. It did this by firstly determining the high priority areas for attention, and then ensuring that they remained pre-eminent throughout the planning process.

Self-reporting by the DHB Planning and Funding Manager indicated good connection between the needs assessment and the prioritisation process, and with the DSP. But only a moderately strong connection with the DAP was reported, because there was not "enough time to bed the strategic plan into peoples consciousness while they were putting together their individual team business plans." There were apparently strong connections with the rest of the planning and purchasing pathway. Regarding the HNA and planning process, the Planning and Funding Manager noted that "It would be about the need to pull planning and needs assessment together." This DHB used bounded 
rationality and a mixed scanning approach together with service planning groups to develop funding priorities for health services, and achieved strong connections between the components of the planning process.

In summary, Hutt Valley DHB assumed bounded rationality and took a mixed-scanning approach to planning. They focused HNAs on a mix of priority government objectives and service areas considered to be high priority in their districts, such as youth, maternity care, primary care, diabetes and disability support services. They also formed service planning groups to develop service plans for the selected priority service areas. This resulted in the HNA having the highest impact for all DHBs on the planning and purchasing process.

\subsubsection{Case study B (Hawke's Bay DHB)}

This DHB was involved with the RHNAP process using the Public Health Consultancy of the Wellington Clinical School. The DHB found three main difficulties with this process, yet recognised considerable benefit. The first difficulty they experienced was in agreeing their approach to HNA. Discussions ranged from the more traditional HFA approach, which they considered was a demand-rationing tool, to broader approaches including service-by-service analysis. The second difficulty was obtaining appropriate Māori involvement and agreement between Māori when there were so many Iwi in the district. The third related to the selection of key informant interviewees and managing geographical representation to obtain a broad range of people, including principals of schools, consumers, Māori health, rural kaumatua, and some providers. ${ }^{56}$

\section{Mixed scanning model}

This DHB also chose to use implicitly, a bounded rationality and a mixed scanning approach for the strategic planning process. Management chose priority areas for planning, but the basis on which they did so is not clear (that is, it is hard to be fully

\footnotetext{
${ }^{56}$ Kaumatua are Māori elders who are able to speak on behalf of Māori Iwi.
} 
transparent in a mixed scanning approach, as there is some 'prior' screening of topics/needs). Eleven service planning groups were established in March 2001 to plan services including primary care, maternity, diagnostic specialist, mental health, disability support services, and others. Each of the service planning groups had up to 10 members, with representatives of providers, consumers, rural communities, and Māori, as well as a child advocate in each group. Public advertisement invited involvement in service planning groups, attracting 250 people replies. DHB staff were also represented by one staff member from the Planning and Funding Department and one from the provider arm in each group. The HNA was due for completion at the same time as the Board's service plans, and initially the groups had to begin work without full HNA results being available. However, the views and opinions of the members of the service planning groups regarding health and service need were reportedly 'validated' by the needs assessment data as they came through. The groups met up to eight times each, and developed service plans that were then consulted on with the community in October 2001.

Representation from the community and NGOs was deliberately dominant in the groups. The rationale for this was made clear:

Hawke's Bay had a pretty public life over the last 10 years with the redevelopment of our facilities and we have learnt things about consultation and involvement of the community. We very explicitly decided that we were going to cut it from the start. (manager)

Planning group members were expected to work up to 20 hours a month for an eightmonth period, but in the event not all groups needed to commit this much time. Recompense for their time "turned it away from a process of community people helping out of a charitable sense, to one where they felt a level of responsibility for making sure they got it right and so the commitment was really good." A Local Reference Group (including representation from Housing New Zealand, WINZ ${ }^{57}$ and Māori Development Organisations) was established to facilitate the project within the DHB, ensure

\footnotetext{
${ }^{57}$ Work and Income New Zealand (WINZ) is the state agency that provides assistance to those seeking work, and income support for those unemployed.
} 
community engagement, facilitate Māori input and participation, access local people and information sources, raise issues, and provide input into the service planning process. In general, the DHB considered that it would not do the HNA and planning process much differently in future, except that they would encourage the RHNAP to coordinate more with inter-sectoral groups.

The DHB reported that consultation with the community during the HNA process occurred implicitly with the involvement of over 80 community representatives in the service planning groups. It also was also reported to have been undertaken explicitly during the interviews with key informants and in consultation on the draft needs assessment. Key informants included Māori health providers and kaumatua, who were all interviewed by Māori. A kaumatua also accompanied each of the Māori interviewers. Provisional needs assessment information was fed back to groups, and feedback from the groups to the needs assessment process.

\section{Target strategies}

As a result of the strategic planning process, eight 'target strategies' were chosen and incorporated into the annual plan. The eight strategies targeted children, Māori health, lower socio-economic groups, avoidable hospitalisations, the impact and incidence of smoking-related diseases (diabetes, cardiovascular, cancer) the prevalence of mental illness, the rate of sexually transmitted infection and teenage pregnancy, and community confidence in the delivery of health services. It is not clear how these target strategies were identified.

The DHB also established some delivery strategies: a major technology programme, infrastructure development for primary health organisations, and intersectoral cooperation. The HNA process made it possible to prioritise the Government's health priority objectives using data from the assessment. The target strategies prepared by the DHB each had indicators against which to measure progress over the next ten years. However, the DHB observed that fiscal constraints meant that the HNA and prioritisation was not used as much as managers considered desirable, particularly 
because no untagged funding was forthcoming from government. The DHB had a significant deficit, but planned to break even within three years, disinvesting where there was duplication of services. The Board had specified that it did not want any service cuts, but would agree to reconfiguration of services.

\section{Prioritisation}

The prioritisation framework was based on that used by the former HFA. The criteria were appropriateness, accessibility, equity, quality and affordability. The DHB intended to prioritise during service reconfiguration, but had not done so to date. A review of diabetes services was under way and the DHB planned to apply the prioritisation framework to those services. Māori were reported to participate in the assessment of competing claims and establishment of priorities at management level through the Funding Management Committee, and at Board level by means of representation on the Board.

\section{Consultation}

Consultation with the community was described as a "double hit." First, full public consultation on service plans was undertaken in Hawke's Bay by means of public meetings and hui. The public was asked whether any significant issues were missing from the plans, and was advised that the DHB would return to consult on the DSP. Feedback from public consultation was recorded and analysed, and, if it met DHB expectations and understandings, was incorporated into the DSP. Secondly the DHB consulted on the DSP, and followed a similar process, including the incorporation of feedback.

\section{Connection on the pathway}

The DHB felt that HNA and prioritisation were connected very well, as were prioritisation and the DSP. "Because of financial constraints" the DAP and DSP were related "not as well as they should be, but they are as far as possible." The budget closely reflected the DAP. 
The challenges of resolving the deficit within three years led to these observations:

The disappointing thing in this whole process is that we have been set up as DHBs, we have got good buy-in by the communities, we know where we need to make the difference but there is such little amount of flexibility that basically everything we can find is already pre-committed or needs to be used for the deficit, so it is quite difficult. ... I think that a linkage up between the HNA processes that DHBs are undertaking, the way the Government sets priorities and the way that the Crown budgeting process is undertaken, probably needs to be strengthened. (manager)

The Board also utilised a Funding Management Committee (FMC) consisting of senior management. This committee was responsible for prioritising health service purchasing in accord with the Board-approved Annual Plan. The influence of the FMC was significant in terms of the actual allocation of funding. The FMC could not allocate funds outside of the DAP, and spending also had to be signed off by the CEO. Allocations greater than $\$ 3 \mathrm{M}$ required Board sign-off.

\section{Comment}

This DHB demonstrated the use of bounded rationality and a mixed scanning approach to planning, using previously identified priority areas for focused planning. This approach was applied with service planning groups involving community, stakeholders, and DHB staff to plan for the DSP. There was consultation during the process, as well as on the DSP. Involvement of staff from the funder, and board members, led to a demonstrable buy-in by community, stakeholders, management, and DHB Board to service plans. The connections between the various elements on the pathway from HNA to purchasing were considered to be strong at all stages, even when the steps did not occur in order. The exception was a weaker - only moderately strong - connection from the DAP to the DSP, which was attributed to funding constraints and a deficit that required 'managing out' over a three-year period. 


\subsubsection{Case study C (Whanganui DHB)}

This DHB is another of the 12 provincial DHBs that used the RHNAP process. Good Health Wanganui, ${ }^{58}$ predecessor to the Whanganui DHB, initially established a needs assessment process with An Assessment of the Health Needs of the Good Health Wanganui Region (Good Health Wanganui, 2000a). Early in the Transitional DHB planning process, the DHB formed a relationship with the Wellington Clinical School and agreed to contract them to conduct HNA. Eleven other DHBs joined the process, subsequently co-ordinated by Technical Advisory Services, a shared services support agency for the Central Region.

\section{Comprehensive rational planning model}

Whanganui DHB followed the comprehensive rational planning model outlined by the Ministry of Health in the recommended planning cycle for DHBs (Ministry of Health, 2000 b, p.6). Health needs were identified in the needs assessment, key priorities established, and then planning proceeded to the DSP and then DAP.

\section{Health Needs Assessment}

The Wellington Clinical School conducted the HNA. A local reference group in Whanganui consisted of community and provider representatives, including local Māori. Engagement with local Iwi was considered sub-optimal, and efforts were subsequently made to improve these relationships. There was no specific consultation with the wider community during the needs assessment process, but 14 key informant interviews were conducted. Data shortages were encountered in primary care and mental health.

58 Whanganui was the original name of the region, but the spelling changed during renaming of the Wanganui settlement, presumed to be around the early 1900s. Whanganui is returning to more common use. This reflects a widespread difficulty with pronouncing and transliterating a particular Maori consonant. 


\section{Prioritisation}

The DHB adopted a prioritisation process using the five principles established by the former HFA, and adding quality as a further principle. Whanganui DHB, like most boards, did not apply the principles when writing the DSP, as the prioritisation framework and principles were to be consulted on simultaneously with the DSP. An exception related to an additional $2 \%$ of government funding to cover population growth in the district. Decisions on purchasing of services using new funds involved using a flow chart to assist decision-making. It required consideration of certain requisite information - whether a proposal was in line with the HNA, DSP, DAP, and government strategy and policy, and also with the results of the consultation process including with Māori Iwi.

\section{Connection on the pathway}

This DHB was clear on the strength of the connection it perceived between components on the pathway from needs assessment through the planning process - the Connection Score was 12, or moderately good. Document analysis gave an Impact Factor of 3.77, which suggested that the actual impact of the HNA on the planning process was consistent with self-reported data.

\section{Comment}

The Whanganui DHB used the comprehensive rational planning model proposed by the Ministry of Health, and achieved a high Impact Factor. Experienced staff and good timing contributed towards the successful outcome. Resources were readily available and the deficit was not regarded as a significant factor. Community involvement was adequate, but the DHB commented that it would have liked more community and Māori involvement. It is clear from the experience of this DHB that the comprehensive rational model can also be applied to incorporate the HNA effectively during the planning process. 


\subsubsection{Case study D (West Coast DHB)}

\section{Health Needs Assessment}

The West Coast DHB has the smallest population of any DHB, at 30,303 people (Census 2001 data), but it was the second smallest in budgetary terms, with an annual budget of $\$ 57 \mathrm{M}$. For the $02 / 03$ year a deficit of $\$ 2.5 \mathrm{M}$ was projected, improving to $\$ 1.2 \mathrm{M}$ in the following year. The West Coast DHB district is the longest geographical region in New Zealand, taking seven hours to drive from top to bottom (Karamea to Haast) and is predominantly rural. For these and other reasons, the DHB suffers from significant diseconomies of scale, a factor that the DHB considers is only partly provided for in the Ministry of Health's funding formula. The Impact Factor for this DHB was 2.75, placing it in the group whose HNA resulted in a low impact on planning. But the Impact Factor was by no means the lowest; it recorded the sixth lowest score.

At the time of the study, some senior management undertook several roles: the Planning and Funding Manager was also General Manager for Mental Health and Primary Services, and also acting Chief Financial Officer for part of the time the HNA was being conducted. The manager observed that not much managerial time was devoted to the HNA exercise, mainly because of competing demands.

The services of the RHNAP were utilised for the HNA, and this was considered to have been most useful. The Board undertook key informant interviews and a provider stocktake. Attention was drawn to the difficulty of obtaining meaningful data when the denominator was small. The DHB consulted with the community during the HNA by holding focus groups with stakeholders, mental health groups, disability groups and others. The assessment revealed no single new key issue. The DHB knew that the key issues were related to access to primary care services, particularly for those with low socio-economic status. The HNA highlighted deficiencies in data regarding mental health, the health status of Māori, and primary health care. The HNA process was felt to be of value to the DHB: 
Well I actually think I am really glad that whoever in Wellington thought about doing this HNA did so, because although I don't think ours was the best of documents, in the end it was a really good start. It is changing the culture of the DHBs to moving away from being provider focused to looking more towards population health and primary health, and that has got to be good.

\section{Prioritisation}

The following comment on the capacity of the DHB to respond to local needs and values is of interest:

On the face of it, health reforms were giving more autonomy to areas to meet the needs of the population. For us on the West Coast, because our total funding is so small, there is little room to move within that funding and we have to meet so many national service frameworks for service delivery, that it is virtually impossible. We can only do it around the edges.

The DHB was quite clear that prioritisation should reflect local needs and values. As for a prioritisation framework, "We borrowed someone else's just to tick the box... It was one done for South Canterbury Health which was done from the Shared Service Agency, which was probably from the HFA one." In practical terms, was it really useful? - "No, not at all useful," but this is at odds with the comment quoted above, indicating inconsistency of the interviewee.

\section{Iwi influence}

The influence of Māori was limited:

We certainly had quite good Māori involvement in the setting of our Strategic Plan which our Annual Plan flows from, but a lot of that to be honest is words, and in real actual terms the influence is probably zero, because of our funding constraints. (manager)

This response is surprising, given that local Māori had a relationship with the DHB and had sought recognition as tangata whenua. ${ }^{59}$ The explanation given for lack of responsiveness seems very convenient.

\footnotetext{
59 Tangata whenua means 'people of the land' and refers to local Māori with tribal connections to the area.
} 


\section{Consultation}

Consultation on the DSP was extensive, with over 30 public meetings, including four hui. The DHB said that HNA and prioritisation, and the DSP, are connected well "on paper", but in practice "not at all". The DAP and DSP were said to be well connected, but the connection was lost between the DAP and the budget. The reasons for this were not altogether clear. It appears that not enough consideration had been given to aligning the budget with the DAP, and that the budget was historically based. Whilst the DSP and prioritisation were consulted on, it appears that the linkages between them were weak.

\section{Comment}

The West Coast DHB achieved an Impact Factor of 2.75, sixth lowest amongst the DHBs. In general terms, this case study raises significant issues: available managerial capacity; health planning experience; timing of the planning process (there was an overall shortage of time and the planning process was short-circuited); and local constraints on the reconfiguring of services - reconfiguration of hospitals in Buller and Reefton has been vigorously resisted, creating obstacles. The DHB originally intended to follow the comprehensive rational model devised by the Ministry of Health (Ministry of Health, 2000b, p.6), but did not do so.

The West Coast DHB had a significant budget deficit, but was actually over-funded by $7 \%$ according to the Population Based Funding Formula, partially offset by a rural premium. Perceptions regarding the deficit size may have influenced planning, but as this research has shown, the impact of HNA on planning for DHBs overall was not linked to deficit size.

\subsubsection{Case study E (South Canterbury DHB)}

The South Canterbury DHB provided health services for a usually resident population of 52,785 . It had an annual budget of $\$ 70.4 \mathrm{M}$ and virtually no deficit. The Impact Factor was 1.41, significantly lower than for any other DHB. 


\section{Comprehensive rational planning model}

The intention was to use the comprehensive rational planning model, but difficulty arose when the DHB was late joining the RHNAP. The time available for planning was consequently shortened, and the outcome was that the DAP was based around the budget, and the DAP and DSP were written simultaneously. The DHB notes that this "obviously was not how you would plan to do it, but the timing this year was very poor". The reality for this board was that the budget was settled first, then the Annual Plan, ensuring that planning was consistent with the available funding.

\section{Health Needs Assessment}

The DHB used the RHNAP; its needs assessment was of a high quality, and was similar in presentation and content to those of other DHBs in the provincial group. The DHB said that it did not have the capacity to conduct needs assessment, and therefore it had outsourced it. Key informant interviews and a provider stocktake were also completed locally, but not outsourced. Māori input was obtained through Māori board members and from one hui. Mental health and primary care data were difficult to obtain.

\section{Prioritisation}

The prioritisation framework and principles were consulted on by the DHB, but were not used in the planning process. The DHB had virtually no deficit, and had no difficulty in meeting its budgetary requirements. Prioritisation appeared to have been undertaken starting with the 13 key priority objectives, and then considering the HNA for any further health service requirements it had uncovered.

\section{Connection on the pathway}

Connections on the comprehensive planning model pathway were significantly disorganised. This must have had a major impact on the planning process, which appears to have been strongly budget-driven. Nevertheless, this is one of the few smaller DHBs that have achieved near-zero or zero deficits, which is highly 
commendable. Maybe there are some lessons to be learnt from this DHB regarding budgetary control.

\section{Comment}

Several factors could have contributed to the difficulties that South Canterbury DHB had in bringing the HNA to bear on planning. The first was timing: the time available to complete the process was curtailed; and secondly, it was not possible to undertake comprehensive rational planning as the planning process had become back to front. The lack of experienced of staff was probably also a contributory factor.

\subsection{Interviews with Ministry officials regarding expectations for health needs assessments and prioritisation}

Interviews were conducted in December 2001 with two Ministry officials who were involved in the policy setting process for HNA and prioritisation. They willingly shared their insights for this research, which are summarised below.

The impetus for establishing DHBs in their present form came from the government's wish for a democratic approach to decision-making in health care. It was intended that boards should focus not only on hospitals, but also on primary care, community services, and the overlap with other non-health areas such as education and housing. The Ministry noted that considerable effort had been made to encourage DHBs to take an intersectoral approach. To do this effectively, DHBs needed to understand the health needs of communities. Within the Ministry there was a push for needs assessment. There was an intellectual precedent for needs assessments as the RHAs previously undertook them. There was also external support for better use of HNA, as previously HNAs had produced "very large, very nice, booklets, which were then not used..." Others had commented, "If you want this to bite, then you will need to place this in the legislation." Therefore the Ministry included HNA in the legislation, alongside the New 
Zealand Health Strategy, in a legally binding framework that made it mandatory. DHBs could therefore spend money on needs assessment, as it was explicitly included in the legislation. It was considered important to preserve the cycle of HNA, planning and purchasing of services, monitoring, and feeding back to needs assessment so as to improve the process effectiveness of health service purchasing.

The Ministry of Health's accountability indicators for DHBs also reflected the importance of HNA and prioritisation, specifically requiring DHBs to work intersectorally and with communities, and especially to listen to voices that had not been heard previously. DHBs were expected to send their HNAs to the Ministry of Health by 1 November 2001. One stated view was that this was a check on the process. Another interviewee stated that the Ministry did not intend this as another "hoop" or "compliance activity" for DHBs, but later contradicted this by saying that it was to see that they were performing the process properly, and therefore was "sort of a disciplinary or control thing". One suggested a less benign interpretation, which was a need to ensure that needs assessments were not being used to "set up Government... by means of a strong incentive to demonstrate significant levels of unmet need as a basis for lobbying."

HNAs were to reflect local need for health services. It was expected that the HNA and subsequent prioritisation would give DHBs the evidence base on which to make their planning decisions, which should also be consistent with the New Zealand Health Strategy. The HNA was designed to give DHBs information as a basis for prioritising their activities, and it was recognised that these might differ from the priorities of the Ministry. It was also recognised that there was a possibility that DHBs might come up with a pattern of services at odds with current provision, and that this represented a political risk to government if major changes to services were made too quickly, rather at the margin. Monitoring of performance by the Ministry would also need to take account of local priorities. 
Another key issue for the Ministry was the importance of incorporation of the findings from HNAs into DHB planning processes and purchasing. It was observed that previously needs assessments had traditionally been performed by one part of the organisation (such as the public health department), but they had not included finance departments, contract departments and the policy section. "The whole DHB needs to get behind it and be guided by it." The Ministry advised DHBs that DSPs and DAPs should refer explicitly to needs assessment work. The Ministry facilitated this by providing a template to DHBs setting out its expectations for the content of DSPs and DAPs.

It was noted that the development process for the New Zealand Health Strategy (NZHS) initially resulted in 61 objectives, reduced to 13 priority population health objectives in the final document. The Ministry assumed that the HNA would allow DHBs to prioritise those 13 objectives appropriately for local conditions, recognising that there would be differences across the country. Some DHBs, faced with a priority population health objective in such areas as nutrition and family violence, would need to give those areas priority particularly if they had no health programmes in these areas.

One unexpected benefit of the HNAs was that the Ministry found that they had 21 reports with extensive and detailed data from the DHBs. The Ministry proposed that in due course these data would inform another iteration of the NZHS, although there was no timetable set for doing this.

Variation in the quality of HNAs was expected, depending on whether DHBs had employed HFA-trained people with experience in HNA.

It was intended that DHBs should use needs analysis to decide what services to deliver. Initially, changes would be expected to occur at the margin. Ultimately the test for DHBs would be whether any services came to an end because of needs assessment. It was considered undesirable if they were basically seen as a planning-for-growth initiative. Are DHBs willing to disinvest where the HNA indicates this is desirable? 
And will they use PBMA to disinvest? Will DHBs follow through and shift resources to areas of higher priority from those of lower priority, and in accord with data from HNAs? In some cases this would mean a shift from delivering services in the hospital to delivering them via primary care. Attention was drawn to an internal conflict of interest: DHBs responsible for the vitality of their hospitals could be confronted by needs assessments that demonstrated a need to transfer resources out of the hospital they own. It was noted that the former RHAs and HFA could genuinely take a detached view of need and could in principle shift resources unfettered by other considerations. Examples included the closure of smaller hospitals such as Dannevirke hospital that was undertaken by the Central RHA in 1997 in response to reduced need for services, and a requirement that more complex procedures be conducted at Palmerston North Hospital (Coster, 1999).

There was also some suspicion that DHBs could manipulate HNAs to achieve their own ends: "Do you fiddle the needs assessments to get easy answers, or do you produce the needs assessment and face the ambiguity of not implementing it?" Achieving a balance in priorities between national consistency on the one hand and local responsiveness on the other was considered to be one of the conflicts at the heart of the current model. This led to a discussion of the contradictory signals given by the present government: previously supportive of a shift from the RHAs to the HFA, they then noted the "folly" of four RHAs having four different sets of priorities. The present Government's position is that there is now a return to a national health system, but that, in addition, it is giving control to local people. It may be that local health needs and priorities will have primacy until such time as there is an awkward public issue for central government, at which point central control is likely to step back in. The challenge for DHBs was seen as how to respond to local needs, given that they can deviate from national uniformity, but only up to the point where deviations become a source of political risk to the government. The importance of building consensus and carrying the public with DHBs in the process was noted, particularly because shifting resources resulted in winners and losers, with excessive media noise resulting in a return to national uniformity. So far, the only example of return to national uniformity as a result of variation between DHBs is that of the criteria for access to surgical procedures, particularly those related to orthopaedic 
surgery. The policy shift from a centralised, HFA to DHBs was concerned with local ownership, and responsibility and decision-making, all within parameters set by Government. This represents an important paradigm shift from the previous HFA where control and decision-making were centralised.

HNAs were to be regularly updated and the NZPHD Act 2000 required DHBs to conduct three-yearly needs assessments. The Ministry expected that additional needs assessments would also be required where there was evidence for further research (currently DHBs are undertaking such work in Disability Support Services, Māori Health, Primary Care and Mental Health). HNAs were to be an integral part of the health planning process, but were not to become an industry in themselves, and so far that has remained that way.

Although HNA and prioritisation were separate processes, the Ministry considered it important that they remained linked, but that the focus should be on prioritisation. It was considered too easy to talk about needs assessment rather than focus on the harder issue of prioritisation. "Talking about needs assessment is easier than talking about prioritisation, because prioritisation equals core health services, equals rationing, equals difficult, so the use of the term HNA is almost a bit of linguistic sanitisation for resource allocation." It was also suggested that some components in the national health strategy were included from pure political expediency and were very hard to justify on any grounds of evidence. It was hoped that DHBs would be more evidence-based in their approach!

\subsection{Discussion}

\subsubsection{Introduction}

One of the key research questions for this thesis was:

\section{What was the impact of the HNA policy on District Health Boards?}

It involved the following sub-questions: 
1. What kinds of HNAs have been conducted by health authorities in New Zealand, and to what extent have they corresponded with official HNA policy goals?

2. What impact have HNAs had on DHB planning processes?

3. Which DHB HNA programmes have been the most effective and least effective, and why?

4. How does the New Zealand experience with HNAs compare with those of other systems and countries?

5. What are the policy implications for health planning, HNA etc? Should the current expectations for, and approach to, HNA be changed, and if so how?

These questions were posed because the New Zealand Public Health and Disability Act 2000 specifically provided for DHBs to conduct population-based HNAs for their districts every three years. The assessment of need had to be taken into account in the district strategic planning process, which was outlined as a comprehensive rational planning model by the Ministry of Health (Ministry of Health, 2000b, p.6). DAPs were required to be consistent with DSPs. This planning cycle was reinforced by the Operating Policy Framework for DHBs provided by the Ministry of Health. The question arises whether such a policy was effective in ensuring that the health care needs of the population were taken into account in the planning process - was the policy effective in securing its intended outcome? What were the policy implications for health planning? Should there be a change of approach? There was some surprise among managers that government should be so specific in stating its requirements for HNA and the incorporation of priorities into DHB planning, so the topic was considered worthy of research. It has subsequently come to light that the focus on HNA was part of the method by which the Labour Government tried to distinguish the 2000 health care system from its predecessor. 


\subsubsection{Types of DHB health needs assessment}

This section covers the types of HNAs undertaken in DHBs in New Zealand and the extent to which they corresponded with expectations, given official HNA policy goals.

The type of HNA conducted by health authorities depends to a certain extent on the definition of need employed. Definitions of need have been much debated (Bradshaw, 1972b, Bradshaw, 1972a, Buetow and Coster, 2001, Carpinter, 1989, Coster, 2000, Liss, 1993, Ministry of Health, 2000b, Wright, 1998). Two thirds of DHBs discussed the meaning of need and defined need as 'capacity to benefit' from intervention (Bradshaw, 1972b). Alternative definitions were not seriously considered. Perhaps in future DHBs will want to debate further the meaning of need and how it applies to their populations.

DHBs have undertaken two forms of population-based HNA to date. Firstly, the Ministry requirement was for population-based HNA for the population normally resident in the geographical district. Most DHBs undertook this approach in accordance with the government policy and gathered demographic and epidemiological data on the population of the district, (Ministry of Health, 2000b). However, three DHBs used bounded rationality and a mixed scanning model, and commenced with priority population health objectives and priority service areas. They formed service planning groups aligned to these and conducted a combination of population-specific and epidemiological HNAs relevant to those areas. This was significantly different from the Ministry's recommended approach regarding the way that HNAs were to be incorporated into DHB planning cycles, yet this resulted in the HNAs having a relatively high impact on planning for these DHBs. The Ministry of Health approved approach assumed that DHBs had no prior knowledge of needs in their districts.

Secondly, DHBs conducted additional population-based but specific HNAs in Māori Health, and also service specific needs assessments in Mental Health, and Disability Support Services. These studies resulted from Ministry of Health requirements for Service Plans for these areas, which required Ministry approval. 
One such service review was the Review of Far North Health Services by an Independent Review Team (IRT) appointed by the Northland District Health Board, with input from the Ministry of Health and the Far North District Community (Clarke, Coster, Reid and Scott, 2002). ${ }^{60}$ The IRT used HNA data from the DHB to define need for health interventions in primary and secondary care, and prioritised disinvestment in some health interventions in order to reconfigure services. Additional data were also collected from the community, including Māori, regarding the requirement for health services. In this case, the HNA data were used in the context of a particular decision. The change process to initiate the reconfiguration required specific data on inpatient and outpatient hospital service loads (case-weighted inpatient and daypatient data) and data on the community's need for health interventions. Health interventions were then prioritised on the basis of effectiveness, equity, value-for-money and binding budget constraints, although this process was not explicitly stated. The result of the Review was that the Northland DHB has planned a reconfiguration of services to meet the reprioritised requirements for health services, and is currently seeking capital approval for the project from the Ministry of Health.

Only recently has there been evidence of other kinds of HNAs originating in DHBs themselves, encompassing such areas as Māori health, mental health, and primary care. DHBs were previously responding to the Ministry-driven agenda regarding populationbased HNAs, and DHBs had almost certainly not considered undertaking any other specific needs assessments, for two reasons. Firstly they had no time, and secondly DHBs had a wealth of information derived from data already collected, except in the areas mentioned. However, DHBs wishing to develop services in those areas are now embarking on new HNAs. It is likely that DHBs will undertake a wider range of focused HNAs in the future that consider specific requirements for services.

${ }^{60}$ The author was a member of this review team. 


\subsubsection{Impact of DHB health needs assessments}

HNA was included in the NZPHD 2000 legislation to ensure that DHBs had a legal basis to fund and regularly update needs assessment. Needs assessments have given the government significant datasets upon which to base future planning, and should have given DHBs a new locus for autonomy (that is, the ability to determine requirements for health services locally), whilst simultaneously informing the national debate on future health services. However, while democratically elected boards were mandated to assess need and prioritise services, the government maintained significant control of national and local agendas, using such levers as Ministry directives, Minister's 'start here' list, NZHS, key priority population health objectives, 38 Health Strategies for boards to implement, the DHB Operational Policy Framework, Service Coverage Agreements, letters of intent and oversight of District Plans. Locally, boards acknowledge local priorities, pressure groups, and political factors, all of which have some influence.

The research shows that the impact of HNAs was variable, with Impact Factors ranging from 1.41 to 5.0 (out of a maximum of 5), mean 3.28, and median of 3.04. Reference to Figure 15 (p.230) suggests that Impact Factors fall into three groups - two DHBs at the top for Impact Factors and Connection Scores; a large middle group of 15 DHBs; and a small group of three DHBs at the bottom for Impact Factor. The group at the upper end were very effective in achieving high Impact Factors and high Connection Scores for their HNAs in their planning process. The larger middle group of DHBs were only moderately effective with their Impact Factors; half of the group had a high Connection Score, but the other half scored low, suggesting some variation between groups in the way that Managers assessed the degree of connection. The smaller group of three DHBs at the lower end had low Impact Factors, yet were satisfied that good connections had been made.

On the basis of the interviews with Planning and Funding Managers, and the data presented in Table 30, p.229, the conclusion drawn is that HNAs were not particularly effective in influencing DHB planning processes. Nor does it appear that the government's expectations outlined in the Ministry officials' interviews regarding HNA have been met. This is not to deny that considerable effort has been made by DHBs to 
date, in difficult circumstances, to achieve cohesiveness in planning. For HNAs to have impact, better connection will need to be achieved in future. The Ministry of Health will need to require boards to take a greater interest in their HNAs and to incorporate the requirements for health services identified through the HNA, into DAPs. Boards will also have to be more proactive in ensuring that identified health need is addressed in plans. Boards will also benefit from a more conscious effort being made at all steps of the planning process to address HNA findings and include them in health service development plans. Boards would also benefit from a service planning group approach, following a mixed scanning based planning and prioritisation process.

Variations in the use of HNA by DHBs contributed to their variable effectiveness. Some DHBs appeared to have used the needs assessment at the DAP stage, but not for the DSP. Others were more consistent in its use, and yet others apparently made little use of the needs assessment. This variation in the planning process, and resulting discontinuity between identified health needs and service priorities in many DHBs, is evidenced by the findings, shown in Appendix 13, p322.

DHBs found that 'global' data collections were useful for obtaining a 'picture' of the health of district populations, and particularly baseline observations; but they were treated as descriptive only and not a guide to action. Wright $(1998$, p.6) points to HNAs as a systematic method of identifying unmet health and health care needs of a population; and he suggests that besides epidemiological data they should also involve qualitative approaches to determining priorities, incorporating clinical, costeffectiveness and patient perspectives. This is based on the assumption that health status alone does not describe health need, which in turn does not describe the requirements for specific health services.

Finance Management Committees (consisting for example of Chair, CEO, GM Finance, and Chief Operating Officer [Hospital]) often made the final decisions on DHB spending, budgets, and deficit reduction, at the end of a long planning and funding process. At this point, it was noted by managers, needs assessment and prioritisation 
had little impact, particularly since Planning and Funding Managers were no longer involved in the decision-making process. Only if needs assessment was used more extensively in the planning process (e.g. by service planning groups) was there any likelihood of impact at the final stages.

The overall national cost of DHB HNAs was $\$ 1.575 \mathrm{~m}$, equivalent to some DHB deficits (03/04). Although many Planning and Funding Managers indicated that HNAs were very worthwhile, particularly for data gathering purposes, HNAs do not appear to represent overall value-for-money in the present context, considering the relatively few outcomes of the HNAs (compared to their potential) as a function of the cost of obtaining the data. The main reasons are insufficient impact on the DHB planning process, lack of prioritisation of services, and a consequent inability to address health need as 'capacity to benefit'. Clearly, HNAs in New Zealand do not occupy a central position in the health planning process. The expense will only be warranted if DHBs make more effective use than at present of the HNA and prioritisation processes during planning in the future.

There is a growing body of research evidence suggesting that bounded rationality and a mixed scanning approach allow HNA to exert the greatest impact on planning and purchasing. This relates to a much wider body of research in public policy that tends to show that comprehensive rational planning approaches rarely 'work'. Kilduff et al (1998) suggest that the starting point for needs assessment should be internally identified areas of concern. Hawe (1996) argues that where service delivery decisions are to be made, HNAs should be targeted selectively to provide the information most likely to affect decision-making. Hensher and Fulop (1999) found that HNAs in London Health Authorities appeared to be guided by a mix of deliberate issue selection and analysis and incrementalist bargaining. Jordan et al (2002), in a study of 67 health authorities in the UK, found a need for careful selection of topics for HNAs. The findings supported the 'mixed scanning approach' where HNA was used to investigate areas previously identified as priorities through a wide-ranging scanning process. 
This present research found that for DHBs planning health service purchasing for the population of their districts, bounded rationality with prior topic selection of priorities was more likely to allow HNA to have an impact on planning and purchasing than more comprehensive global approaches. In future, DHBs should take more focused approaches to HNA, in terms of district-wide planning and in refining topic selection for more detailed activity. This latter amounts to a mixed scanning approach, and involves a two stage process consisting of firstly selection of likely topics, and secondly more detailed work on priorities arising from the first part in order to formulate a working list. Clearly, DHBs will also have to continue conducting population-based HNAs, as they are required to do so by legislation.

In summary, population-based HNAs by DHBs were: of lower impact or effectiveness than expected, without realistically delivering value-for-money; of variable use and quality; health profiles more than definitions of requirements for services in many cases; and largely ignored by managers during the final process of decision-making. The context for this lack of effectiveness included an inability to terminate existing health service contracts, and an unwillingness to reprioritise using cost-effectiveness to guide decision-making. In addition, any reduction of health services required the specific approval of the Minister of Health. These issues represented significant problems for the use of HNAs in the future and wider problems for any notion of comprehensive rational planning by DHBs.

\subsubsection{Most effective and least effective health needs assessments}

There were significant differences between the effectiveness of DHBs' HNAs in influencing the planning process. This section draws on those experiences and presents a catalogue of features that were significant factors in those differences.

Two DHBs that used a mixed scanning model of planning were among the top group of three for positive impact of HNAs. One other DHB also used a mixed scanning approach and was highly rated. The findings suggest the need for careful initial selection of priority topics, using a wide-ranging scanning process, for planning and 
action from the range of priorities identified by the government and the DHB as important. During the scanning phase these DHBs took care to ensure that planning and prioritisation took place in areas that were important and productive. Priority areas were chosen from Government priorities, and further work areas selected by the DHB Boards/management, taking account of national priorities. Projects formed in these areas were then populated with HNA data and became the subject of planning, service evaluation, and prioritisation.

Where DHBs used mixed scanning approaches, more focused collection of reliable and valid HNA data allowed insights into problems, and the identification of solutions. Needs assessment was used to investigate areas identified as priorities by a more wideranging process. It could be argued that needs assessment had a greater impact in these more focused DHBs because there was already a determination to make a change or to do something, prior to or even irrespective of the needs assessment. However, there was no evidence from the interviews with the Planning and Funding Managers that this was the case.

However, a number of other boards achieved relatively high Impact Factors using the comprehensive rational model described by the Ministry (2000b, p.6). HNAs gave rise to key recommendations that were then prioritised by boards and carried forward into DSPs and DAPs. It is apparent that both the mixed scanning and comprehensive rational planning models can achieve a high impact of the HNA on the planning and purchasing process. However, the mixed scanning approach appears to have been particularly successful and is therefore recommended to be given serious attention by all boards when preparing District Plans.

Those DHBs that used a mixed scanning approach also all used service planning groups to plan services for each of the priority planning areas. These have been described previously (for example see Case study A, Hutt Valley DHB, p.234). Service planning groups were not used by the other DHBs involved in the 'comprehensive planning model' advised by the Ministry of Health. 
The research has shown that large amounts of data need not necessarily be collected to plan health services successfully. There were two issues. Firstly, 'global' or populationbased/comprehensive approaches were broad in scope, but more focused approaches were found to be successful in achieving the connections between the components of the planning pathway. Secondly, there was a lack of focus on the 'requirements for services' - that is to say the HNAs were just 'health profiles' or health assessments. There appears to be no reason as to why such data cannot be made available through a central process in the future, using a central resource similar to the RHNAP project. Such a resource would be of assistance to DHBs undertaking more focused HNAs, and allow ongoing monitoring of the health of the population of New Zealand. Furthermore, the data could be collected on a five-yearly basis, to coincide with the five-yearly Census, thus ensuring current data. For the above reasons, the author suggests that DHBs do not undertake 'global' population-based HNAs in the future, but rather focused HNAs instead, and that data gathering for five-yearly health assessment be conducted centrally, fitting with the Census cycle.

Those DHBs that did well in the planning process were more likely to have experienced planning managers compared to those that did less well, insofar as the impact of HNAs on DHB plans was concerned. On the other hand, there were some experienced planning managers in DHBs where the HNA did not achieve as much impact over the planning outcomes. These observations need to be interpreted carefully, as they are based on the planning manager experience among the 'top five' DHBs compared to that of the 'bottom five'. As indicated above, there are a number of other factors that are important in achieving impact of HNAs on DHB planning processes.

It should also be remembered that DHBs were new at the time when HNAs and DHB planning were first conducted. Boards took time to establish functionality, with new responsibilities, structures, and organisational cultures to establish, especially alongside significant personnel changes. The closure of the HFA meant that some former HFA managers became co-workers in DHBs, with resulting tensions. Some hospital managers also changed roles and took on new responsibilities for non-hospital services, including primary care, with which they had little or no previous experience. This 
represented a significant culture change for these previously hospital-focused managers. Whilst difficult to quantify, organisational and functional change no doubt had a significant effect on the ability of management to plan and manage in the new environment, and to incorporate HNA into the planning process. That the DHBs did only moderately well using needs assessment in planning can in small part be attributed to organisational change and the newness of DHBs.

Timing was also a significant factor. Those boards in the more effective group reported that they were able to undertake steps in an appropriate sequence to achieve their goal. One DHB (South Canterbury) joined the RHNAP project late, which had significant adverse effects on planning timeframes and its ability to impact the planning process. Most DHBs were focused on their DAPs, given that management of their budgets and deficits was clearly linked to the DAP. For most boards, the DSP was developed alongside the DAP, and in some cases, subsequent to the DAP.

Most DHBs found it difficult to complete HNAs and planning within the time allowed by the Ministry of Health, particularly as the planning process was a new experience for many. Some DHBs did not establish clear objectives, which would have facilitated the process.

In summary, key differences between the most and the least effective HNAs were the use of 'bounded rationality' and a 'mixed scanning approach'; the use of service planning groups; the experience of managers; timing factors and timeframe; and organisational process. Attention will have to be given to these factors if HNA is to become more relevant to the planning process than it is at present. 


\section{Chapter 8: Conclusion}

\subsection{Summary of research findings}

\subsection{Conclusions}

\subsection{Research process}

8.3.1 Strengths and limitations of the research

\subsection{Recommendations for policy}

\subsection{Future research}




\subsection{Summary of research findings}

This research focused on the impact of HNAs on planning processes before and after the creation of DHBs, particularly regarding DHB planning and purchasing.

The findings of the research are presented below in relation to the research questions:

\section{What kinds of HNAs have been conducted in health authorities in New Zealand, and to what extent have they corresponded with official HNA policy goals?}

\section{Pre-DHBs (1991-2000)}

This part of the research evaluated HNAs conducted by the Ministry of Health, Public Health Commission, and health authorities including the RHAs and the Health Funding Authority, between 1991 and 2000. It evaluated the impact of HNAs on decisionmaking, service delivery, and health policy. It also considered what barriers there might be to the implementation of the findings from HNAs during this period. HNAs in New Zealand evolved over the last 22 years from a more or less infrequent activity in Area Health Board planning, to become a regular activity within RHAs and the HFA, and are now mandated by the NZPHD Act 2000 for DHBs purchasing health services for their populations.

According to the typology adopted for the purpose of the research (see Chapter 3, p.68), of the HNAs conducted between 1991 and 2000, 58\% were population-based, 10\% community-based, $12 \%$ epidemiologically-based, $8 \%$ comparative, $12 \%$ corporate, and none economic. The majority of HNAs were undertaken by the Regional Health Authorities (34\%) or the Ministry of Health (26\%). Help with decisions regarding the purchasing of health services was the key objective during the decade in $54 \%$ of HNAs, but despite this, only $48 \%$ of reports made any recommendations at all, despite all the HNAs having objectives. Objectives of HNAs were seldom clearly stated at the outset 
but were generally included within the body of the report somewhere, either directly or by inference.

The types of HNA reports in the sample chosen for in-depth interviews (1997-2000) differed from those over the decade only in there being a greater proportion of population-based HNAs (72\% versus 58\%) in the sample. During the years 1997-2000, decision-making regarding the purchase of health services was the key objective of a similar proportion of HNAs (48\%). Fully one quarter of the reports were for research purposes. During this period only $44 \%$ of HNAs led to an action regarding health service purchasing, 36\% led to a service review with no change, and no action was taken as a result of $20 \%$ of the studies (that is, nothing happened as a result of those studies). A needs assessment was more likely to lead to policy action when the priority emerged from the analysis of local data or when detailed needs assessment focused on issues of local relevance (for example, service planning). These data were similar to those of Hensher and Fulop (1999). However, they found that needs assessment directly supported decision-making and action in two-thirds (66\%) of the London studies examined, compared with New Zealand's 44\%.

Although there was little difference between the stated objectives of HNAs of the 19972000 group and those for the whole decade, there were some significant differences from those of the London health authorities studied by Hensher and Fulop. Those from London were more likely to be undertaken to resolve service-specific purchasing decisions (31.4\% versus 10\%), and less likely to be undertaken for research purposes ( $14.1 \%$ versus $26 \%$ ), or for other primary objective ( $2 \%$ versus $12 \%$ ), or for multiple purposes $(9.4 \%$ versus 36\%). These data suggest that HNAs in England were more focused in their objectives, which is not altogether surprising since their health authorities have been undertaking HNAs since the internal market reforms of the NHS in 1991. 


\section{Post-DHBs (2001-)}

DHBs each conducted a population-based or 'global' HNA as required by the Ministry of Health. Only recently, some have begun collecting more focused needs assessment data on Mäori health needs, mental health service needs and/or Disability Support Services. None of these were completed in time to be included in the research. However, this is the beginning of the evolution of HNAs in the right direction towards more focused approaches. This thesis evaluated the DHB's population-based HNAs. HNAs conducted pre-DHBs corresponded with official HNA policy goals, although they were not articulated well under the Area Health Boards. Within DHBs, HNAs have mostly corresponded with Ministry of Health policy goals, except that they were more like 'health profiles' than HNAs. Three DHBs used a bounded rationality and mixed scanning approach as opposed to comprehensive rational planning from a zero-base, and formed service planning groups for defined priority health objectives and service areas (discussed in more detail later). These DHBs undertook some prior screening of issues/priorities and identified key services and/or areas for further work. They then formed service planning groups for each of these, populated the groups with a mix of staff, providers, Māori and community representatives, and provided focused HNA data relevant for the service to each of those planning groups. It should be noted that the service planning groups and HNA were not separated but integrated. Although this approach was different from the comprehensive rational approach prescribed by the Ministry, it was highly effective, and consequently has implications for planning policy.

The study found that clear objectives, decisive leadership, teamwork, communication, sound study design, adequate resourcing, skilled staff, sufficient time, community engagement and consultation (where appropriate), consultation with Māori, 'ownership' by and involvement of stakeholders (including funders), and linkages with the implementation process were all important in ensuring that HNA influenced health service purchasing. Apart from the requirement for consultation with Māori, these findings were similar to those regarding HNAs conducted by the London health authorities (Fulop and Hensher, 1997, Hensher and Fulop, 1999, London Health Economics Consortium, 1996). 
The DHBs did not debate the meaning of health need to any significant extent in their HNAs, and not at all in one third of cases. The New Zealand definition of HNAs refers to 'capacity to benefit from health care services', and this may have influenced DHBs to produce 'health profiles' rather than HNAs. It is probable that in view of the information provided to DHBs regarding the definition of need as 'capacity to benefit', that DHB managers decided that they need not debate the meaning of 'need'. However, in order to better focus HNA on the issue of 'need', the author suggests that Frankel's reference to 'requirements for health services' is a more suitable wording for this part of the New Zealand definition of HNA (Frankel, 1991a, p.257). Furthermore, as 'effectiveness' is included within the term 'cost-effectiveness' there seems to be no reason that both terms should be included separately in the definition of HNA. The revised definition would then be: 'Health care needs assessment is the assessment of a population's requirements for particular health care services, prioritised according to relative cost-effectiveness, and funded within available resource.'

In general, DHBs met the Ministry of Health's minimum requirements for HNAs as set out in accountability indicator GOV-02 (see Box 2). However, there was considerable variation in the quality of HNAs: when HNAs were evaluated using the 'gold standard' Ministry requirements, augmented by other criteria based on the international literature, Quality Scores ranged widely between 44 (for those in the RHNAP process) down to 17 for one DHB, out of a possible maximum score of 46.

The degree of engagement with and participation by Māori varied considerably, from none to full consultation. In some cases, the involvement of Māori staff of the DHBs was considered sufficient to obtain a Māori perspective. Some DHBs had difficulties securing the participation and engagement of Māori because a relationship with local iwi was not in place, and took time to establish. A number of DHBs stated that, in retrospect, they would have preferred to engage with Māori earlier and better. This would have assisted in obtaining a better understanding of Māori health needs. 
Almost without exception, DHBs found difficulty in obtaining and validating data. Data most difficult to obtain were primary care (sometimes because IPAs and GPs would not release data), mental health services, and Māori health data (owing mainly to a lack of ethnicity coding). Every DHB commented on the poor quality of ethnicity data generally, especially related to primary care.

Most DHBs found that the process and outcomes of the HNA were valuable, although some DHBs commented that the HNA did not highlight a single new issue. HNAs were seen to provide a base of information on which to plan health service delivery. Almost every DHB saw the HNA as a means of reviewing the progress of the board, and planned to track changes in the health status of the population over time, (that is, to use the results of the HNA for ex post monitoring, rather than for needs assessment and service planning).

\section{What impact have HNAs had on DHB planning processes?}

Every DHB reported that they found the process and outcomes of the HNA were useful, although some DHBs commented that the HNA did not highlight a single issue that they had not known before. HNAs were seen to provide a solid background of information on which to plan health service delivery. Almost every DHB saw the HNA as a means of reviewing the progress of the Board, and planned to track changes in health status of the population over time (that is, using the HNA for ex post monitoring, rather than prospective need assessment).

The impact of HNAs on the planning process was assessed in two ways. Firstly, an Impact Factor was developed on the basis of documentary analysis of the connections that DHBs achieved between the priorities revealed in their HNAs, the priorities agreed to by boards, and the importance subsequently attached to those priorities in the DSP and DAP. A mathematical approach was used to determine Impact Factors for each board, using document analysis to collect data. Secondly, a Connection Score was formulated on the basis of the Planning and Funding Manager's assessment of the connections between HNA, board priorities, DSP and DAP for each DHB. 
The research showed that the impact of HNAs was highly variable, with Impact Factors ranging from 1.41 to 5.0 (the maximum being 5), with a mean of 3.28 , and a median of 3.04. Reference to the plot in Figure 15, p.230, which demonstrates the relationship of Connection Scores to Impact Factors, suggests that DHBs fell into three groups - two DHBs with Impact Factors and Connection Scores at the top; a large middle group of 15 DHBs; and a small group of three DHBs at the bottom for Impact Factors. Overall, the Planning and Funding Managers' self-assessed Connection Scores did not suggest particularly good connections across the planning process. The conclusion drawn is that the HNAs were not particularly effective in influencing the DHB planning processes, except for those of two DHBs that did exceptionally well.

Analysis of the prioritisation principles chosen by DHBs found that a total of 36 different principles were selected by the DHBs, the average number of principles adopted by each board being 6.5 (range $4-11$ ). Of the 20 DHBs analysed, 10 included all five of the principles set down by the former HFA (equity, acceptability, effectiveness, Māori health, and cost), but only three boards did so exclusively. Boards freely expanded on the range of HFA principles, and at least 10 boards omitted some or all of those principles. Interviews with Planning and Funding Managers indicated that the boards did not proceed to apply their prioritisation frameworks with their new principles to any significant degree. Even if boards had applied the prioritisation frameworks, there would be conceptual difficulties in working with 6-7 principles at any one time. Issues for consideration include how to ascribe explicit meanings and values to principles, quantifying those values, and then undertaking an analysis that accurately reflects the different weightings that elected boards wished for prioritisation. There was considerable variation among the DHBs' prioritisation frameworks, and if the frameworks are used in the current forms there may be significant variation between DHBs regarding access to health services as a result. Boards agreed on priorities for services based on the New Zealand Health Strategy and other strategies, the Ministry's priority service areas, the Minister of Health's 'start here' list, the Operational Policy Framework from the Ministry of Health, and lastly the boards' own preferences. Funding management committees held the real decision-making power in a number of Boards. These committees consisted of the CEOs, a few key managers and sometimes 
the Chair, and they determined the final shape of Annual Plans. DHBs received strong messages that the Government's priority objectives had to be reflected in DSPs, and it appears that this was an overwhelming influence on decision-making.

Almost all DHBs considered that prioritisation should apply only to new funding where it was not tagged, and all stated (accurately or not) their perception that over $99 \%$ of their budgets were pre-determined by existing contracts with health providers that they had inherited. It is very likely that this will change in the future as contracts come to an end and boards allocate resources on the basis of the DSP. Some DHBs undertook a line-by-line approach to budget review, seeking to identify non-performing contracts, and opportunities to amalgamate contracts to achieve economies of scale. Those boards that did so reported that they were able to release only a small amount of funding through this process.

There were high expectations that the first round of HNAs and prioritisations would make a significant impact on DHB planning and purchasing. That this did not happen can be attributed to global population-based approaches to HNA (that is, lack of prior filtering/prioritisation and lack of focus for HNAs); the comprehensive rational planning model required by the Ministry of Health; the extent of Ministry of Health directives; major organisational and staff changes occurring simultaneously within DHBs; lack of experience with the new planning processes; lack of use of/familiarity with prioritisation/re-prioritisation, including health economic approaches; general lack of experienced planning staff; a compressed timeframe; and timing/sequencing of planning for DSPs and DAPs which should have been conducted in that order, but were not necessarily so. Ministry staff who were interviewed considered that the tension between national consistency in priorities on the one hand and local responsiveness on the other was one of the conflicts at the heart of the current model of devolved purchasing with national upward accountability of DHBs.

DHB managers estimated that the overall national cost of HNAs was $\$ 1.575$ million during 2001/02. Considering the relatively few outcomes of the HNAs compared to 
their cost, HNAs do not appear to represent high value-for-money. The main reasons are that they have insufficient impact on the DHB planning process; that many HNAs amounted to 'health profiles' or health assessments rather than HNAs; and a failure to prioritise services results in a consequent inability to address health need as 'capacity to benefit'. ${ }^{61}$ Clearly, HNAs in New Zealand do not occupy a central position in the health planning process. The expense will only be warranted if DHBs can make more effective use of HNA and prioritisation during planning processes than they do at present.

Health assessment data should therefore be collected centrally on behalf of DHBs. This thesis research showed that on grounds of both cost and quality that the centralised Wellington Clinical School project was most effective. DHBs would benefit from a centralised resource for health assessment data. Use of outdated data during the last round caused many DHBs to rework their data in order to reflect Census 2001 data. Health assessment data collections should therefore be done on a five-yearly cycle, to match Census data collections.

\section{Which DHB HNA programmes have been the most effective and least effective, and why?}

DHBs that used a mixed-scanning model of planning were amongst the group whose HNAs had the most positive influence on planning. This finding suggests the need for careful selection of priorities for planning and action, using a wide-ranging scanning process, from those regarded by the Government and DHB as important. Whilst the comprehensive rational planning model recommended by the Ministry of Health was also successful, it was less likely to be so than mixed scanning approaches. Given the constraints, the comprehensive HNA / rational planning model is not very helpful, and wastes time and resources.

\footnotetext{
${ }^{61}$ A health profile is a description of the health status of the population, using epidemiological methods. This does not equate to a health needs assessment, which has a greater focus on the health needs of the population.
} 
Global population-based approaches to HNAs in districts were more like 'health profiles' or health assessments than HNAs. Internationally, it is now uncommon to find comprehensive HNAs conducted on the whole population of a district or geographical area, as it has been found that focused needs assessments (which are service/clientspecific) are more likely to be useful in planning processes.

The following features determined which DHBs exhibited better connections between their HNAs, priorities, DSP, DAP, and purchasing were: the planning model they chose (mixed scanning was particularly effective); their experience in health planning; the use of service planning groups (sometimes called service development groups); the involvement of stakeholders (including the community); and the timing of the processes. The size of the DHB's population had no effect on the success in the process; nor had the size of the deficit as a proportion of total budget.

The quality of the HNA itself did not correlate with its impact on the outcomes of the planning process. As indicated in the discussion in section 7.10.4, DHBs that did well in the planning process were more likely to have experienced planning managers compared to those that did less well. This is true regarding the 'top five' and the 'bottom five' DHBs, but there were a number of exceptions to the rule, and clearly planning experience is only one of the factors that determine the outcome of the planning process. A number of lessons have been learned as a result of HNAs conducted by health authorities and these should assist DHBs with HNA and health service planning in the future.

\section{How does the New Zealand experience with HNAs compare with those of other systems and countries?}

New Zealanders commonly have an ideal of an egalitarian society and are generally familiar in health with the meaning of need and have a concern for health inequalities, especially for Māori and disadvantaged groups. Therefore it was surprising to find a low consciousness of the different definitions of 'health need' among DHBs, as evidenced from the HNA reports. Maybe managers decided that it was easier not to debate the 
meaning of need than to enter into a time-consuming discussion where it might be difficult to achieve agreement. The Ministry of Health guidance and literature review probably pre-empted that discussion.

There are significant differences between the typical use of HNA in New Zealand and its international use, where assessment is most commonly focused on specific services. Most DHBs in New Zealand avoided the issue of need by just describing the health of the population. The effectiveness of existing DHB HNAs was limited because the prevailing approach focused on health profiles, and took little account of requirements for health services.

Frankel suggests that ambiguous references to HNA can be avoided by using the term 'health care requirements', rather than 'health needs', to refer to the desirability of providing particular services (Frankel, 1991a). By this subtle change in terminology, he makes plain the link to the purchasing of health services. Levels of health care requirement depend not just on the prevalence of a condition, but on the prevalence of the condition where treatment would be indicated, is acceptable and desired by the person potentially receiving the treatment, and is agreed by experts and the community to be an acceptable use of the health budget. The term 'health care requirements' focuses thinking beyond 'health need' and may be useful to help managers conceptualise the need for services. DHBs ignored this distinction, almost certainly because the Ministry had framed the discussion around 'health need', rather than 'health care requirements', in its advice to DHBs. But the guidance had made it clear that need equates to 'capacity to benefit' (from a service).

The process by which an issue is selected for the HNA, and the context in which it is undertaken, can be crucial to its subsequent influence on action (Hensher and Fulop, 1999, Jordan, Wright, Ayers, Hawkings, Thomson, Wilkinson and Williams, 2002). Hensher and Fulop (1999) surveyed 217 HNAs in 14 London Health Authorities and concluded that the place of needs assessment in practice is consistent with the "mixed scanning model' of decision-making. Mixed scanning approaches involve scanning the 
environment to choose appropriate areas in which to begin gathering intelligence on the basis of some prior knowledge or experience. Care must be taken during this phase 'to identify those issues that impose a significant disease burden and from which service change might plausibly achieve substantial benefit, and to minimise the adverse impact of high profile or special interest issues...' (Hensher and Fulop, 1999, p.94-5). This means early problem definition and a prior theory of improvement being possible. During the 'scanning' phase care needs to be taken to focus planning and prioritisation activity on areas where the management time required will be productive. The choice of areas for study by needs assessors can be influenced by pressure groups, government priorities, and political factors. Some of these factors may lead to bargaining in order to achieve resolution. Such an approach is more consistent with the incrementalist, bargaining model of decision-making. In New Zealand, the choice of areas to focus planning and prioritisation activity will be determined by such factors as the NZHS priority population health objectives, the Minister's 'start here' list, local board priorities, local pressure groups, and other political factors, and a sensible DHB would start in these areas.

In England, Australia and New Zealand, similar themes emerged as important considerations for the effective conduct of HNAs: clear objectives; rigorous methodological design; involvement and buy-in by stakeholders; planning model; and adequate timescales. Timing of HNAs to fit into the DHB planning process and the experience of planning managers were also key factors in ensuring that HNAs had an impact.

As discussed in Chapter 2.7, p.61, international experiences showed that HNAs did not occupy a central position in health service decision-making and had only a small impact on planning and purchasing. In reality, the greater part of public health service purchasers' budgets are already committed, leaving only a small proportion of funding available for developing new or different services according to need. But this should not be an impediment to effective HNAs if they are targeted on expenditure that can change, and supports the case for focused HNAs. The New Zealand experience is no different, and if this is to change, then some hard decisions will have to be taken 
regarding the place of population-based HNAs. Either a greater commitment must be made to incorporating the findings of HNAs into DHB plans, or the activity should be curtailed, at least in its present form. Focused approaches have been shown to be the most useful, and should be the norm for HNA and planning even if DHBs, government, and the Ministry still require 'global' health profiles as data gathering exercises to inform future policy. Such profiles could be compiled and collated centrally on a fiveyearly basis, to coincide with the Census cycle, as discussed in section 7.10.4, p.262.

The international trend, as discussed in Chapter 2.12, is for HNAs to be focused, rather than broad-brushed, global population studies. There is no international evidence that global population-based approaches are more effective than focused approaches, and it is now uncommon to find HNAs conducted on the whole population of a geographical area and for all services/needs. In England and Australia needs assessment activity is focused; and it needs to become more focused in New Zealand if it is to fulfil its potential. For example, it is assumed in England that Primary Care Trusts have access to a health profile of their populations, but this is not equated with HNA. New Zealand could improve the effectiveness of HNA by ensuring that focused HNA data are incorporated into a planning process that uses service planning groups. Such groups would be formed following a mixed scanning phase where deliberate scanning is used to ensure that issues for closer focus are chosen appropriately.

\section{What are the policy implications for health planning, HNA etc? Should the current expectations for, and approach to, HNA be changed, and if so how?}

The current planning model is not working. The comprehensive rational planning model is unrealistic and fails to recognise the inability of planners to collect and process the necessary information in the real world. There are significant constraints on DHBs as indicated above, and the comprehensive HNA/rational planning model is not helpful, and consumes time and resources. Therefore, given that there is a high degree of central Ministry control over decision-making by boards, the need for population-based HNAs being conducted by boards is questionable. Instead, the opportunity exists to refocus the use of HNA by replacing the current planning process used by most DHBs to more focused activity on issues of national and/or local concern. Mixed scanning is not 
intrinsic to New Zealand's public health system, but if the present 'over determined' system is to change, then the mixed scanning model of selection of priority areas for planning offers DHBs a new approach to more closely align planning with local need.

A way will have to be found of conducting transparently the first stage of choosing a small number of areas/priorities for focused HNAs from the larger range examined in a mixed scanning model. As long as a reasonable case for particular foci chosen can be made, then DHBs should be well placed with their deliberations. Choosing should also be easier as time passes and DHBs gain local knowledge and experience. Once this is done, boards can then introduce more robust prioritisation processes to follow HNAs, including health economic approaches that consider equity, effectiveness, value-formoney, and binding budget constraint. This approach will focus on resource shifts at the margin in high/low priority areas.

The health planning workforce is mobile and requires augmentation with trained health planners. There is also a shortage of health economists who can provide expert assistance with prioritisation using health economic tools, with estimations of costeffectiveness of different health interventions relevant to health programmes and services. Such cost-utility analyses could be done centrally for New Zealand, similar to those conducted by the National Institute of Clinical Effectiveness (NICE) in England. Further recommendations are discussed in section 8.3.

\subsection{Conclusions}

This thesis considers the impact of health needs assessment on planning and purchasing in the public health sector in New Zealand. The research design and organisation (see Figure 9, p.132) illustrates the two arms of the study, being the impact of 1991-2000 and DHB HNAs. It is timely to draw together the conclusions of the thesis to illuminate our understanding of health needs assessments from 1991 onwards and why they have apparently not had more impact in New Zealand. 
The research found for 1991-2000 that NZ HNAs were twice as likely to be used for research purposes (26\% vs $14.1 \%$ ), four times as likely to be used for multiple purposes (36\% vs 9.4\%), and only a third as likely to be used for resolving service-specific purchasing decisions (10\% vs 31.4\%), compared to those in the more experienced London Health Authorities. Furthermore HNA in England was 50\% more likely to support decision-making and action (66\% vs $44 \%$ of HNAs). During this period in NZ just under two thirds of managers and researchers were conducting HNAs for the first time.

The main reason for low impact of HNAs during 1991-2000 appears to relate to the lack of service planning and purchasing specificity. Instead, a rather more diffuse research and population-based approach was taken to answering some broad policy questions, particularly regarding inequalities. As noted previously, these data suggest that HNAs in England were more focused in their objective, which again was not surprising given their experience in the NHS reforms of 1991.

The HNAs conducted by DHBs were all population-based, by virtue of the requirements of the NZPHD Act 2000. They had less impact on planning and purchasing than had been expected (median Impact Factor 3.28, range 1.41-5.0) but DHB Planning and Funding managers rated the HNA connections with the planning process slightly higher (median Connection Score 11, range 8-13.5, max. possible 15). The main reasons behind the relative lack of impact of the HNAs are organisational (need for clear objectives, decisive leadership, teamwork, communication, sound study design, adequate resourcing, skilled staff, sufficient time, and ownership amongst others by internal stakeholders). Organisational values were not tested in the research but circumstantial evidence suggests that these may have been important. The relationship between organisation, information and outcomes of research into policy will be discussed shortly. DHBs found that HNAs provided a solid background of research information on which to plan service delivery. As stated earlier, almost every DHB saw the HNA as a means of reviewing the progress of the board, and planned to track changes over time (that is, using the HNA for ex post monitoring, rather than for 
prospective needs assessment). This approach does not auger well for implementation of current HNA research into practice.

The question that must now be asked is, Why is it that despite similar barriers to HNA in England are NZ HNAs having less impact? referring to HNAs both pre- and postDHBs. The HNAs conducted in England are different from those in NZ. They are more focused, more often service-specific, and are conducted by more experienced managers and analysts. Organisational values and factors may also have a part to play. There is a culture of use of HNAs in policy-making and planning in England, where research data is implemented into policy/planning. So far in NZ we have failed relatively in implementing the findings of research into planning and purchasing. Information is not the problem; it is what we do with it that is.

This leads to a brief comment regarding planning approaches, which have been discussed in depth earlier. Both the comprehensive rational planning model, and the 'mixed scanning' approach were used by those DHBs that were more successful in achieving an 'impact' of health needs assessment on the planning process. However, the finding that 'mixed scanning' appears to have been particularly successful for planning means that DHBs should consider this approach in the future. Furthermore, the finding that focused health needs assessments undertaken in the context of working groups planning specific services had impact, means that DHBs should also consider using this approach in future planning of services.

Two other comments need to be made, regarding the interpretation of the meaning of need, and the conceptual frameworks for prioritisation. The theoretical model of health needs assessment adopted in New Zealand assumes 'need' as 'capacity to benefit' and that 'health care need' refers to the need for health care services. The evolving actual model of needs assessment is one of population-based 'health profiles', which do not fully take account of need, or demand, or ability to supply. Absent from the stocktakes of morbidity and mortality, and of provision of services, are the views of consumers regarding requirements for services. DHB health needs assessments were mainly based 
on epidemiological data from existing sources (for example hospital discharge data), which reflect current usage rather than identify need. Effectively, a gap exists between what is known about current service provision and community need. This is a problem for the existing model. As was argued earlier in the thesis, the theoretical New Zealand model for health needs assessments would be improved by using the term 'requirements for particular health services', which includes the notion that the community wants the service, 'experts' agree that it should be provided, and that the purchaser can provide the service for the person(s) concerned.

The conceptual frameworks for prioritisation by most DHBs were based on the HFA principles of effectiveness, cost, equity, Maori health and acceptability. However, wide variation exists between DHBs in the actual prioritisation principles selected (see Table 27, p.210). A total of 36 different prioritisation principles were selected by boards with the range being 4-11, and the mean being 6.5. If DHBs actually apply their prioritisation frameworks under the current model for prioritisation of services, it will lead to considerable inter-DHB variation regarding access by individuals to health services, with resulting inequities. Although most DHBs clearly recognised government health priorities, they based annual plans around existing budgets, rather than using their prioritisation process. The Ministry of Health acknowledged the potential difficulties and subsequently the National Health Committee has been piloting a prioritisation methodology to provide a common platform between DHBs that incorporates health economic approaches.

The aspirations for health needs assessment and prioritisation have not been realised within the present reforms that are intended to allow communities greater say in their health services through democratically elected health boards, public board meetings and use of health needs assessment and prioritisation of services in the planning process. Only modest incorporation of HNA findings occurred, with scant evidence of reprioritisation to meet unmet need. The research identified a number of barriers that contributed to the reduced impact of health needs assessment on planning and purchasing. But the reforms are only recent and it therefore cannot be expected that strong connections will be achieved in the first planning cycle, yet there are sufficient 
concerns regarding population-based approaches that suggest that they are unworkable. The context is also one of significant central control by the Ministry with national prioritisation of health services through national strategies, priority population health objectives, and Ministers 'start here' lists, as well as Ministry directives meaning that local responsiveness is difficult. A shift of the locus of control towards DHBs would provide greater opportunity for local responsiveness, yet key government priority objectives could still be achieved.

This thesis contributes to our knowledge of the impact of health needs assessment on planning and purchasing by identifying a number of factors that are significant barriers to the process (see p.273). The research finds that the tension between nationally set priorities determined by the Ministry of Health and local priorities determined by DHBs are at the heart of the current model of 'devolved purchasing' with upwards accountability. This research also contributes to our knowledge by the strong implication that mixed scanning approaches to planning health services were uniformly more effective than comprehensive rational planning models. Apart from the present statutory requirement for population-based health needs assessments by DHBs in New Zealand, the research found that planners would be better positioned in planning services by focusing on defined priority services (such as mental health, Maori health, primary care, disability support services), populating the planning project with relevant health needs assessment data, followed by prioritisation and the balance of the planning process. What has been learned is that large population-based health needs assessments in general do not work, and that focused health needs assessments are more effective for planning purposes. It was found that prioritisation/reprioritisation is difficult, even with new funding. Although health economic approaches are clearly needed, only one DHB undertook cost-effectiveness studies and it is concluded that at the present time such approaches will require greater support to DHBs before they are widely adopted.

The predominant disciplines utilised in health needs assessment are those of epidemiology and social science research. Whilst the use of epidemiology needs no further description, the place of social science research is less well understood. It allows us to enlarge our understanding of human behaviour and society. It provides hard data 
for planning, evidence of need and for resources, and can be used to inform planning and policy making. Health needs assessment is situated in the wider context of the relationship between research and policy/planning. Needs assessors doubtless believe, as social scientists, that the utility of their social research rests on its potential as a rational guide to policy/planning. Weiss (1997) states that this is buttressed by interest in the status and rewards that accrue, desire for influence within the organisation and the enthusiasm for policy shifts in the direction of their own beliefs. However, if the findings of health needs assessment are not attention seeking, social scientists soon find that initial interest of policy makers wanes due to competition for attention. Conversely if needs assessment indicates that a change in direction is required, implementation of the research threatens the status quo, and the research findings risk rejection, particularly by those who have a vested interest in existing arrangements. That, combined with healthy scepticism from operational managers based in hospitals, can lead to undermining of the standing of needs assessment research findings. This may explain why Planning and Funding managers reported difficulty in connecting the findings of health needs assessment through to the chain of the planning process.

No social science research is value-free and acceptance of the findings of health needs assessment is to a certain extent dependent on similar value sets of decision-makers and researchers. It was not possible to explore this dimension during the research, but differences in values may have been involved in the planning process. Another difficulty is that the findings of health needs assessments are made at the start or research end of the decision-making process rather than at the policy/planning end. The dilutional effect by the end of a planning cycle invariably means that the chances of successful implementation of research into planning or policy are greatly reduced. This is particularly the case in comprehensive rational planning where needs assessment and the Annual Plan are at opposite ends of the pathway. The 'mixed scanning' approach shortens the process by acquiring social research to order to address a priority area. Some observers have called this a decision-driven model of research. Such research generated in these circumstances is more likely to influence the final policy/planning, compared to more nebulous approaches. 
In New Zealand, the factors that are obstacles to transforming social research results into policy/planning decisions are: differences in experiences and perspectives between needs assessors and policy makers/planners, divergence in values, the unstable and ambiguous nature of social policy research, and the distance that decision-making lies away from initial health needs assessment. These represent organisational or institutional barriers to prioritisation, rather than inadequate information.

These two perspectives on the barriers to prioritisation were debated by Klein and Williams at the 1998 International Conference on Priorities in Health Care. Allan Williams argued that inadequate information was the main problem, while Rudolf Klein argued that the main problem was inadequate institutions. In New Zealand, health needs assessments by DHBs produced a plethora of information and it could be argued that lack of CUA information held up prioritisation. On the other hand that seems unlikely, as DHBs were not clamouring for health economic data. More likely, the problem lies within organisations/institutions, particularly the DHBs, but also the Ministry. Klein argues that institutions matter more than information and 'given conflicting values, the process of setting priorities for health care must inevitably be a process of debate' (p.20). He notes the importance of getting the institutional setting of the debate right, the value of all voices being heard, that there is 'no one value or principle from which we can derive our health care priorities' (p.21) and that the problems of policy making do not stem from lack of information, but from 'lack of consensus about how to use and interpret it' (p.22). DHBs found it hard to engage in the debate around prioritisation, frequently citing that they had no new money of any proportion to prioritise. This was not a statement of lack of information but a reflection of organisational unwillingness, or inability to undertake prioritisation. This was independent of any prioritisation framework, or principles, selected by DHBs. It means, as Klein states, that there is a need to 'strengthen our institutional capacity to analyse evidence, to clarify policy choices and to promote informed debate' (p.24). It seems that our collective ability to make use of the information that is available is inadequate. The argument therefore is that we need to strengthen DHBs as decision-making institutions rather than clamour for more information. 


\subsection{Research process}

Qualitative research has much to offer. Its methods can, and do, enrich our knowledge of health and health care. (Mays and Pope, 2000, p.52)

\subsubsection{Strengths and Limitations of the research}

\section{(a) Strengths}

The use of both key informant interviews and document analysis using a range of documents provided depth and breadth to the study, and made it possible to triangulate results. Interviews obtained valuable insights that would not have been elicited by a questionnaire. Perceptions and understandings were explored in a non-threatening environment where interviewees could speak freely. Managers were remarkably frank and open, and provided explanations and meaning to some of the findings from the document analysis. Surveying 20 of the DHBs in New Zealand yielded a full range of DHB perspectives. ${ }^{62}$ Analysing documents pertaining to the HNAs, prioritisation frameworks, board priorities, DSPs and DAPs of the DHBs also gave important insights into DHB planning processes and the role of HNAs in those processes.

Triangulation of a large quantity of data brought new perspectives to understanding the processes within DHBs. Document analysis suggested that some interviewees had downplayed their DHB's relative success in making needs assessment influence the planning process. Conversely, document analysis also provided a more sober view of the achievements of several DHBs than that offered by some interviewees.

Interviews with managers and researchers involved with HNAs from 1997 to 2000 provided additional insights regarding the documentary analysis of HNAs from the latter part of the 1991-2000 decade. Interestingly, both sets of interviews gave rise to similar findings, which suggests that we need to become better students of history and

\footnotetext{
${ }^{62}$ Note that Waikato DHB did not agree to be part of the Health Reforms 2001 research until late in 2003.
} 
learn from the lessons of the past regarding HNAs. All the respondents in these studies were participants in HNAs, not merely observers, which brings validity to their observations and experiences. They shared knowledge as 'insiders' and their narratives shed light on the realities of HNA and the harsher realities of the DHBs' planning process. Their willingness to share is testimony to their commitment to the health service in New Zealand, and their recognition that evaluative research can make an important contribution to shaping and reshaping the way that we deliver health services. There is no doubt that insights gained from such analysis of people's experiences of health planning can be used to inform policy, and as Kearns proposes, 'New theory and research will emerge from a close attentiveness to narratives found in the context of every day life.' (Kearns, 1997, p.273)

The use of five Case Studies, representing higher and lower HNA Impact Factors, proved valuable in considering the planning processes used and the outcomes achieved. This led to a wider analysis of planning models and allowed conclusions to be drawn on the usefulness of approaching health planning from the perspective of bounded rationality.

The approach to the research questions posed in this thesis was largely qualitative, but quantitative methods were used in several areas, especially to calculate Impact Factors, and multivariate analysis was used to test the relationships between Impact Factors, Connection Scores, Quality Scores and DHB deficits. The case for judiciously combining qualitative and quantitative approaches in health services research is argued by Barbour (1999). In this research, qualitative work was enhanced by using quantitative techniques to analyse data and triangulate findings from separate qualitative studies.

\section{(b) Limitations}

The views expressed by the Planning and Funding Managers on the connections between HNA, the priorities of boards, DSPs and DAPs are subjective. Only one person in each DHB was targeted for interview; personal bias could have been introduced and 
their views may not have been representative. However, sometimes two, three or even four persons were actually present at the interview, and a group view was thus obtained. Furthermore, these managers were all members of the senior management teams at their DHBs, so it was appropriate to attribute some reliability to their views.

The research analysed the impact of the first population-based HNAs conducted by the new DHBs. The analysis could not take account of the possibility that, with experience, DHBs may be able to improve connections between HNAs and the planning process.

The research did not evaluate the accuracy of the data in the HNAs. It evaluated the quality of HNAs against the standards required by the Ministry of Health, as specified in advice to DHBs and in the Health Needs Assessment for New Zealand: Overview and Guide (Ministry of Health, 2000b). Arguably, the overall accuracy of the needs assessments was an important area for evaluation, but this was beyond the scope of the research. However, as much of the data for HNAs were provided to DHBs from national datasets, it was reasonable to assume that they were of high quality. Furthermore, the research has demonstrated that 'quality' was not associated with the likelihood of being used.

The other important weakness lay in the fact that HNA, DSP and DAP documents for the 20 DHBs were analysed by just one researcher to obtain data for the Impact Factors, where ideally several people should have evaluated them independently and then reached a consensus. There might have been opportunities for differences in interpretation. On the other hand, whether or not health needs and priorities were expressly mentioned in each document is reasonably unambiguous, so the analysis should be very repeatable. Even if there were minor differences, they would be unlikely to have any influence on the overall conclusions drawn from this research. And, of course, research for the purposes of a thesis had to be done independently. 


\subsection{Recommendations for policy}

The following brief recommendations for policy are made on the basis of the results of this research:

1. Health care needs assessment at DHB level should be redefined as follows: "Health care needs assessment is the assessment of a population's requirements for particular health care services, prioritised according to relative cost-effectiveness, and funded within available resource."

In New Zealand, each DHB should be concerned to provide or fund an appropriate level of service to achieve the improvement, promotion and protection of health, and provide support for those in need of health services, including those who are disabled. The focus should be on the population's need for specific services rather than on the general health status of the population. Confusion arises when the term 'need' collapses these two concepts (Frankel, 1991b). Is the population in need, or the service needed? A more strongly service-based interpretation is required where the use of the term 'need' causes us to pay attention to the requirements for health care. This modification of the previous definition (Coster, 2000, Ministry of Health, 2000b) clearly links HNA to resource allocation. The term 'effectiveness' is included within 'cost-effectiveness' and the latter term only need be used within the definition.

2. Global population-based approaches to health assessment should be conducted centrally in order to improve quality and reduce costs to DHBs. They should be undertaken every five years to fit with the five-yearly Census cycle so that current census data can be used. Health profiles can be updated annually as new epidemiological data becomes available.

The New Zealand experience showed that most of the HNAs amounted to health profiles, consisting of health assessment data. The research showed that populationbased HNAs did not significantly impact DHB planning and purchasing, certainly not to 
the degree that was expected, and did not represent value-for-money. New Zealand has the opportunity to refocus the use of HNA by changing the current planning process used by most DHBs to one of more focused activity, as discussed above. Health profiles for DHBs should be produced through a central process, and be updated on a regular basis as new epidemiological data comes to hand. The population denominator data can also be updated regularly with the five-yearly Census data. This information can then be regularly provided to DHBs, to use in association with focused HNA activity. Health assessment data can be used for monitoring the impact of service changes brought about by HNA, planning, and prioritisation.

To implement these recommendations, DHBs and the Ministry will need to agree to an amendment to the NZPHD Act 2000 regarding changing the frequency of district wide population-based HNAs to five yearly. Public Health Intelligence in the Ministry of Health could provide the necessary data to DHBs for the five-yearly HNAs.

3. HNAs conducted by DHBs should be focused on specific services, population groups, or areas to allow planners to respond to specific needs identified. Planners should note that both mixed scanning and comprehensive rational models were used by the DHBs whose HNAs achieved a significant impact on planning, but that mixed scanning was more consistently successful, and is therefore recommended.

This thesis research, and international experience, have shown that large populationbased HNA data collections are not as effective in influencing planning processes as more focused data collections. The application of bounded rationality in a mixedscanning approach allowed planners to respond to identified need more effectively. In the DHBs that used this approach, the influence of HNAs on planning and purchasing was observed to be greater than it was in others.

Planning should ideally commence with a set of priorities developed by the DHB after scanning the environment for priority areas, such as NZHS, NZ Disability Strategy, Primary Health Care Strategy, Minister's 'start here' list, Ministry directives, and any 
local priorities. The process for the future may be: prioritisation $->$ focused HNAs in priority areas -> requirements for particular health care services -> reprioritisation -> resource priorities -> plans etc. HNA data can then be collected using a focused approach, to provide data relevant to the priority service planning area to be considered by the service planning group.

Each year DHBs should establish 3-4 focused service planning groups to review priority service areas. The deliberations of these groups would contribute to the ongoing planning process, and to the 3-5 yearly District Strategic Plans. The implementation issues for DHBs in respect to this recommendation are not difficult at all, as DHBs are already undertaking this approach (2004). Focused service planning groups are now located in many DHBs in such areas as primary health care, Māori health, and mental health services. There is no doubt that this focused approach ha become an ongoing activity and that DHBs in the future will develop further service specific planning groups, based on a more 'mixed-scanning' approach to HNA.

4. Sufficient time should be allowed by DHBs during the planning of DSPs and DAPS to allow service planning groups to consider the requirements for health services emerging from HNAs, to prioritise them, and to incorporate them into plans.

Experience has shown that it is necessary to allow sufficient time to the consider requirements for health services that are indicated by any HNAs undertaken, to prioritise them in service planning groups, and to prepare DSPs and DAPs on the basis of the results. In the future, HNA and prioritisation are likely to be conducted on an ongoing basis by DHBs, where focused activity is undertaken on three or four service areas each year, according to need. This means that there is a regular cycle of HNA and planning being undertaken by DHBs. Many of the Planning and Funding Managers reported that timeframes for preparation of plans were tight, and that outcomes would have been better if more time had been allowed. Smaller DHBs have capacity issues in this regard. 
5. Prioritisation and reprioritisation should be regular activities in DHBs, using nationally agreed core principles (such as effectiveness, equity, value-for-money, and binding budget constraint). ${ }^{63}$ PBMA provides a feasible approach to analysing programmes for prioritisation. Prioritisation frameworks should contain a workable number of prioritisation principles rather than the 6-7 principles on average agreed by boards.

In order to ensure fairness and equity of access to health services, a national set of prioritisation principles should be agreed, with boards being able to respond to these according to local priorities. Otherwise, it is possible that significant variation in access to health services may result. Such prioritisation principles should be few enough to ensure that prioritisation frameworks are workable, yet also ensure that desirable principles are included. The Ministry of Health has recently proposed the set of four prioritisation principles referred to in the Recommendation 7 above, and these seem appropriate.

Health economic approaches to prioritisation will require DHBs to develop a greater understanding of effectiveness and cost-effectiveness, including cost / QALY, than exists presently. At present the necessary expertise is not widely available, so it will be necessary to use a central technical resource. In some cases it may be possible to draw on data from NICE, ${ }^{64}$ English National Service Frameworks (NSFs), and WHO$\mathrm{CHOICE}^{65}$ at programme and sub-programme level. In New Zealand, data sources such as the Health Technology Assessment service in Christchurch, and the Ministry toolkits available on the Ministry of Heath website, should also be of use. PHARMAC has a considerable capability for health economic analyses, and this resource should be drawn

63 Principles proposed by the Ministry of Health and DHBs Prioritisation Group (unpublished, but presently being trialled in several DHBs, see earlier discussion).

64 National Institute for Clinical Excellence (NICE) (2003) Technology Appraisals (Completed Appraisals). http://www.nice.org.uk/cattal.asp?c=153, accessed 26 April 2003.

${ }^{65}$ CHOsing Interventions that are Cost-Effective World Health Organisation (2003) WHO-CHOICE. http://www3.who.int/whosis/menu.cfm?path=evidence,cea\&language=english, accessed 26 April 2003. 
on. It is understood that the Ministry of Health will recommend increased resourcing of health economic studies in the near future. The health economic workforce is inadequate in New Zealand at present and additional resources will be required to train more health economists including for DHBs and the Ministry of Health.

\section{The Ministry of Health should consider reducing the central control of prioritisation} if HNAs are to be useful. This may be less of a problem if HNAs are more focused.

The roles and responsibilities of the Ministry of Health include central accountability to government for performance of the health system within the available funding. The Ministry is accountable for the government's priorities in health being delivered by DHBs, as well as partly by the Ministry itself. While the centre has accountability to government, DHBs also have local accountability for governing and funding of local health services. Therefore, the system is not a pure model, but is one of dual accountability of both the Ministry of Health and the DHBs.

Priority setting is a key responsibility of the centre that is also responsible for ensuring a level of national consistency. This is an appropriate model where there is central funding and national strategies, but at the same time it should permit greater opportunity for priority setting to reflect local priorities than occurs presently. The dynamic of central control versus local autonomy has presented an interesting challenge for DHBs. However, DHBs found that they did not have much flexibility to respond to the needs and priorities of their communities. Some rebalancing of the locus of prioritisation may provide DHBs with greater opportunities for marginal change to allow local priority setting to reflect local priorities. 
7. Objectives, methods, recommendations and requirements for particular health services should be clearly presented in all HNAs.

Many HNAs did not include these elements, or they were unclear. Objectives should be clearly stated to obtain stakeholder buy-in. The methodologies used, the sources of data, and its reliability should be explained. Recommendations and requirements for particular health services that emerge from the HNA should be clearly recorded for use in the next stage of prioritising by service planning groups.

8. Training sessions should be provided for Planning and Funding Managers where models of best practice are shared.

Those DHBs that did well in the planning process were more likely than others to have experienced planning managers. It appears that the presence of experienced managers is likely to promote an effective planning process, in which HNAs influence DHB plans. On the other hand, there were some experienced planning managers in DHBs that were less successful in this respect, and clearly there were other factors contributing to the outcome. It would be particularly useful if training sessions could be provided for Planning and Funding Managers where models of best practice from 'successful' DHBs were shared to improve planning outcomes.

9. In health, we should be more careful to learn from the lessons of the past ahead of major policy change.

Significant lessons were available from those who conducted HNAs during the years 1991-2000. If we were better students of history, then the narratives and lessons of those health needs assessors would have helped to avoid some of the pitfalls of DHB HNAs. Hopefully, we will learn from the present findings. 


\subsection{Future research}

This thesis has focused on the impact of HNAs (particularly those undertaken by DHBs) on planning and purchasing in the public health sector in New Zealand. It is important that the Ministry of Health and DHBs take up the recommendations above and implement them to improve the impact of HNAs. DHBs are soon to enter the second round of HNAs conducted on a three-yearly cycle, and ideally the recommendations should be implemented in time for the 2005/06 DAP planning process (the 2004/05 planning round is near completion). Future research should be directed towards evaluating the effectiveness of policy shifts in HNA, prioritisation and planning by DHBs.

There are a number of research questions that need answering once the next planning round is complete: Has the HNA guidance changed? How do boards fairly and transparently choose services for detailed review during the next cycle? Has HNA changed/developed? Has it become more focused? Are HNAs having more impact? What are the outcomes? Are resources shifting within DAPs? Are the government priorities still dominant? Do DHBs still say that they have no room to move? How do we know whether the DHB autonomy/decision-making model is working? These will be the subjects of ongoing research.

Summarising, the main areas for future research are the locus of control - central versus local - in health service planning, the impacts of HNAs on DHB planning and purchasing, how priority areas for HNA are chosen, the effect of central levers used to determine health priorities, reasons for the unresponsiveness of the DHBs regarding prioritisation, and the fairness of the health system, particularly regarding equity and access to services, according to need. 


\section{Appendix 1: District Health Boards, Populations and Geographical}

\section{Areas}

Table A1: District Health Boards, populations and geographical areas

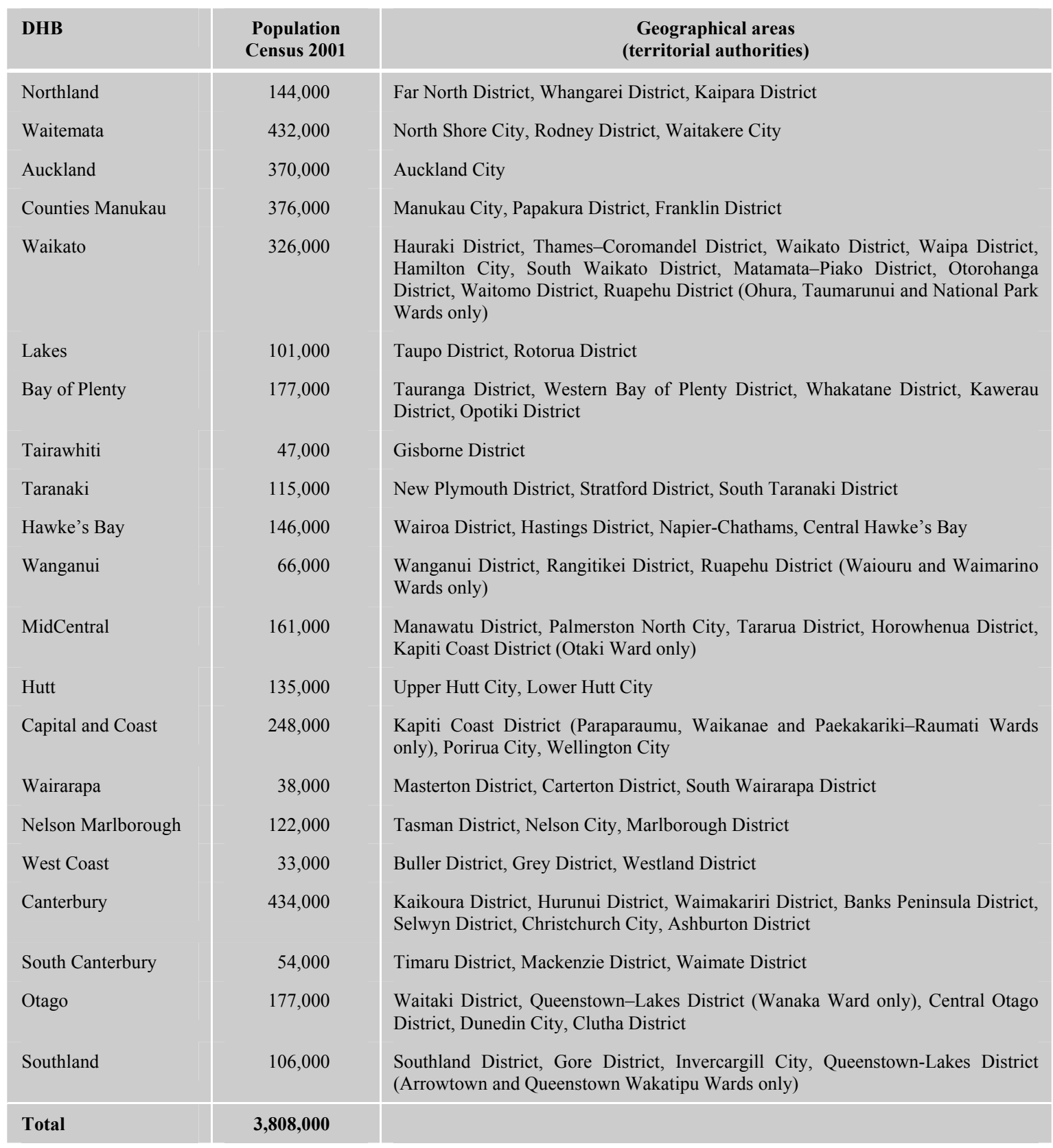

Source: Statistics New Zealand, Census 2001 data. Totals have been rounded to the nearest 1000 . 
Appendix 2:

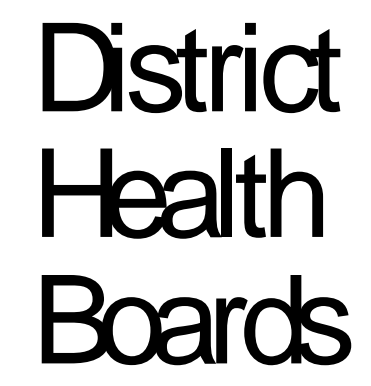

District

Health

Boards Map

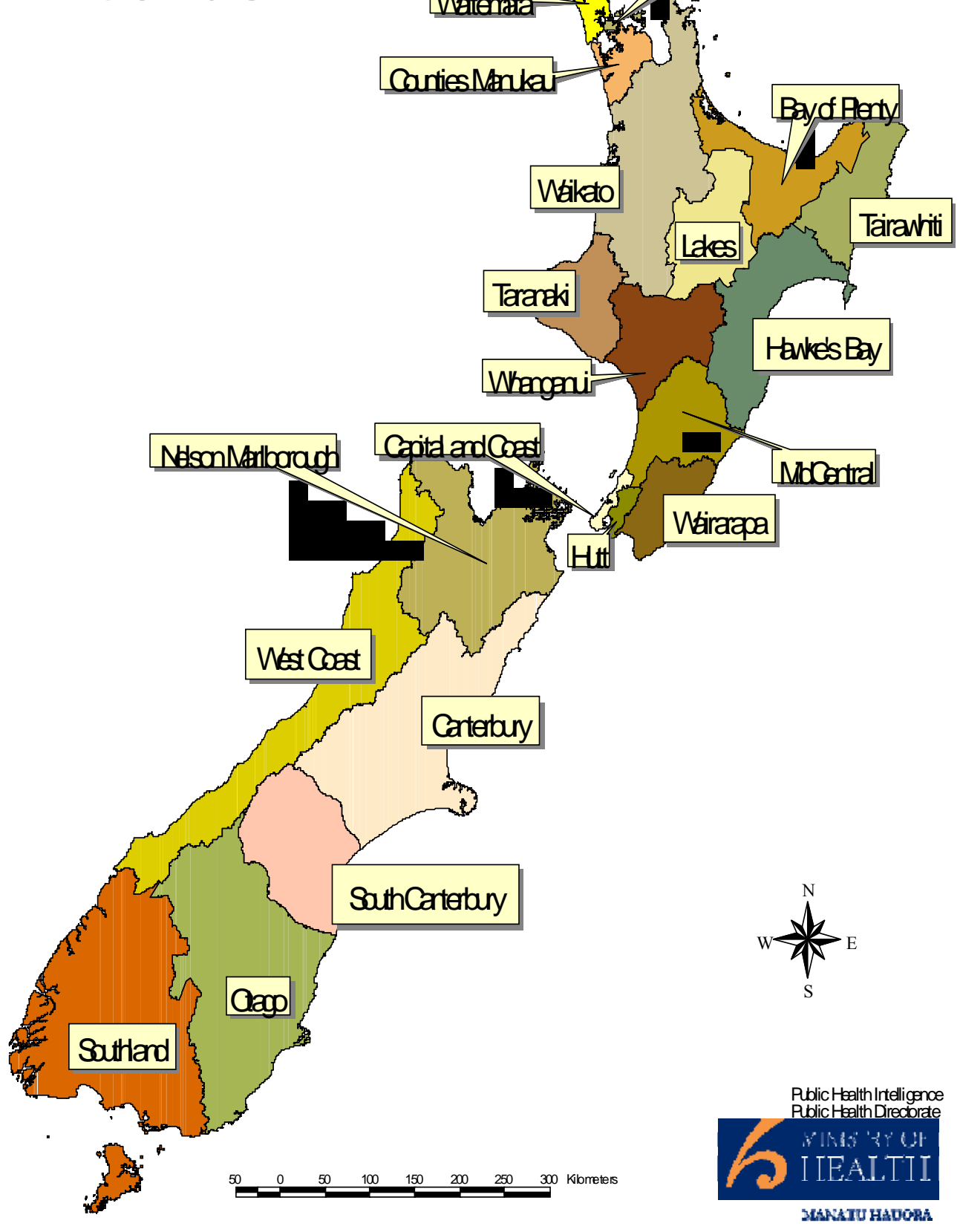




\section{Appendix 3: Health Needs Assessments (1991-2000)}

These have been conducted by the Ministry of Health and the Health Funding Authority and predecessors over the decade, $1991-2000$. Key to Classification: 1 = Population-based; 2 = Community-based; 3 = Epidemiological; 4 = Comparative; 5 = Corporate; Key to Objectives: $\mathrm{a}=$ Service-specific purchasing decisions; $\mathrm{b}=$ General purchasing decisions; $\mathrm{c}=$ Measurement for research purposes; $\mathrm{d}=$ Other primary objective; $\mathrm{e}=$ More than one of the above; $\mathrm{f}=$ Community involvement; $\mathrm{g}=$ Statutory consultation;

Key to Recommendations: $\mathrm{aa}=$ Ministry of Health; $\mathrm{bb}=$ Health Authority; $\mathrm{cc}=$ Ministry of Health and Health Authority; dd = Ministry of Health, Health Authority and other agencies; ee = National Health Committee and Health Authority; ff = Not clear to whom recommendations apply; gg $=$ No recommendations made. $\bullet=$ Interviewed (1997-2000)

Table A2: Past New Zealand HNAs (1991-2000)

\begin{tabular}{|c|c|c|c|c|c|c|}
\hline \multirow[t]{5}{*}{$\begin{array}{c}\text { Inter- } \\
\text { viewed } \\
(1997- \\
2000)\end{array}$} & $\begin{array}{l}\text { Classifi- } \\
\text { cation }\end{array}$ & Title & Author and year & Service area & $\begin{array}{l}\text { Objecti } \\
\text { ves }\end{array}$ & $\begin{array}{c}\text { Recom } \\
\text { mendati } \\
\text { ons }\end{array}$ \\
\hline & 1 & 1992 Health Status Review & (Bay of Plenty Area Health Board, 1992) & $\begin{array}{l}\text { Demographic and epidemiological } \\
\text { approach to health status of various groups }\end{array}$ & $\mathrm{c}$ & gg \\
\hline & 2 & $\begin{array}{l}\text { Adolescent sexual practices: a study of sexual } \\
\text { experiences and service needs among a group of New } \\
\text { Zealand adolescents }\end{array}$ & (Brander, 1991) for Department of Health & $\begin{array}{l}\text { Sexual practice, experiences and service } \\
\text { needs in adolescents }\end{array}$ & $\mathrm{c}$ & gg \\
\hline & 2 & $\begin{array}{l}\text { Women living with HIV/AIDS: issues and needs } \\
\text { confronting women with HIV/AIDS }\end{array}$ & $\begin{array}{l}\text { (Brander and Norton, 1993) for Ministry of } \\
\text { Health }\end{array}$ & $\begin{array}{l}\text { Needs of women with HIV/AIDS and those } \\
\text { caring for them }\end{array}$ & e & aa \\
\hline & 1 & $\begin{array}{l}\text { Strong links: building better services to meet the health } \\
\text { and disability support service needs of people in } \\
\text { Porirua }\end{array}$ & (Central Regional Health Authority, 1994) & $\begin{array}{l}\text { Community health services - organisation } \\
\text { and administration }\end{array}$ & $\mathrm{b}$ & $\mathrm{bb}$ \\
\hline
\end{tabular}




\begin{tabular}{|c|c|c|c|c|c|c|}
\hline $\begin{array}{c}\text { Inter- } \\
\text { viewed } \\
(1997- \\
2000)\end{array}$ & $\begin{array}{l}\text { Classifi- } \\
\text { cation }\end{array}$ & Title & Author and year & Service area & $\begin{array}{l}\text { Objecti } \\
\text { ves }\end{array}$ & $\begin{array}{c}\text { Recom } \\
\text { mendati } \\
\text { ons }\end{array}$ \\
\hline & 1 & $\begin{array}{l}\text { Poutama whirinaki = Interwoven paths: the report of } \\
\text { the Central RHA Wanganui Needs Assessment }\end{array}$ & (Central Regional Health Authority, 1996) & $\begin{array}{l}\text { Health services needs and demands, health } \\
\text { status and socioeconomic factors }\end{array}$ & e & $\mathrm{bb}$ \\
\hline & 4 & $\begin{array}{l}\text { Report on resource equity for the people of the Lakes } \\
\text { Sub-Region }\end{array}$ & $\begin{array}{l}\text { (Cheung and Health \& Disability Analysis } \\
\text { Unit, 1996) for Midland Health }\end{array}$ & $\begin{array}{l}\text { Health resource need of the Lakes region, } \\
\text { access to and availability of health services }\end{array}$ & $\mathrm{b}$ & $\operatorname{gg}$ \\
\hline & 3 & $\begin{array}{l}\text { Prophylactic treatment for severe haemophilia A: an } \\
\text { assessment of the costs and benefits. }\end{array}$ & $\begin{array}{l}\text { (Coopers \& Lybrand, 1995) for Ministry of } \\
\text { Health }\end{array}$ & An assessment of the costs and benefits & $\mathrm{a}$ & aa \\
\hline & & $\begin{array}{l}\text { Strategic directions for the mental health services for } \\
\text { Pacific Island people }\end{array}$ & $\begin{array}{l}\text { (Crawley, Pulotu-Endemann and Stanley- } \\
\text { Findlay, 1995) for North Health }\end{array}$ & $\begin{array}{l}\text { Needs and directions for mental health } \\
\text { services for Pacific Island peoples }\end{array}$ & $\mathrm{a}$ & $\mathrm{bb}$ \\
\hline & 2 & $\begin{array}{l}\text { An investigation into the special health care needs of } \\
\text { refugees for the Auckland Area Health Board }\end{array}$ & $\begin{array}{l}\text { (Deloitte Ross Tohmatsu, 1991) for } \\
\text { Auckland Area Health Board }\end{array}$ & Special health care needs of refugees & $\mathrm{e}$ & $\mathrm{bb}$ \\
\hline \multirow[t]{3}{*}{$\bullet$} & 1 & $\begin{array}{l}\text { Health care needs assessment study: South Island West } \\
\text { Coast }\end{array}$ & (Dunt, 1999) for Health Funding Authority & Health care needs assessment & $\mathrm{b}$ & $\mathrm{bb}$ \\
\hline & 1 & $\begin{array}{l}\text { The people of the Midland Health Region, Volume 2: } \\
\text { Health Status, Part 1: Infants and Children }\end{array}$ & $\begin{array}{l}\text { (Health \& Disability Analysis Unit Midland } \\
\text { Health, 1995) for Midland Regional Health } \\
\text { Authority }\end{array}$ & $\begin{array}{l}\text { Health status of infants and children in the } \\
\text { Midland region- detailed analysis }\end{array}$ & e & $\mathrm{bb}$ \\
\hline & 1 & $\begin{array}{l}\text { The people of the Midland Health Region, Volume 2: } \\
\text { Health Status, Part II: The Health Status of Young } \\
\text { People }\end{array}$ & $\begin{array}{l}\text { (Health \& Disability Analysis Unit Midland } \\
\text { Health, 1996) for Midland Regional Health } \\
\text { Authority }\end{array}$ & $\begin{array}{l}\text { Health status of young people in the } \\
\text { Midland region- detailed analysis }\end{array}$ & e & $\mathrm{bb}$ \\
\hline$\bullet$ & 1 & Family Health Services in the Midland Region & $\begin{array}{l}\text { (Health \& Disability Analysis Unit Midland } \\
\text { Health, 1997a) for Midland Regional } \\
\text { Health Authority }\end{array}$ & $\begin{array}{l}\text { Analysis of family health services- detailed } \\
\text { analysis }\end{array}$ & $\mathrm{e}$ & $\mathrm{bb}$ \\
\hline - & 3 & $\begin{array}{l}\text { Sexual and Reproductive Health in the Midland Health } \\
\text { Region, Volume 2, Part III }\end{array}$ & $\begin{array}{l}\text { (Health \& Disability Analysis Unit Midland } \\
\text { Health, 1997b) for Midland Regional } \\
\text { Health Authority }\end{array}$ & $\begin{array}{l}\text { Sexual and reproductive health in the } \\
\text { Midland region- detailed analysis }\end{array}$ & $\mathrm{e}$ & ee \\
\hline - & 3 & Pregnancy \& Childbirth in the Midland Region & $\begin{array}{l}\text { (Health \& Disability Analysis Unit Midland } \\
\text { Health, 1998) for Midland Regional Health } \\
\text { Authority }\end{array}$ & $\begin{array}{l}\text { Pregnancy and childbirth in the Midland } \\
\text { region- detailed analysis }\end{array}$ & $\mathrm{e}$ & $\mathrm{bb}$ \\
\hline$\bullet$ & 4 & $\begin{array}{l}\text { The health of the people in the south: West Coast, } \\
\text { Canterbury, Otago, Southland }\end{array}$ & (Health Funding Authority, 1998a) & $\begin{array}{l}\text { Epidemiology, health status, needs } \\
\text { assessment data, population characteristics } \\
\text { and health services need }\end{array}$ & e & $\operatorname{gg}$ \\
\hline • & 5 & Kia Tu Kia Puawai: Mental Health & (Health Funding Authority, 1999) & Māori Mental Health & e & gg \\
\hline - & 1 & $\begin{array}{l}\text { Disability in New Zealand: Overview of the 1996/97 } \\
\text { Surveys }\end{array}$ & $\begin{array}{l}\text { (Health Funding Authority and Ministry of } \\
\text { Health, 1999) }\end{array}$ & $\begin{array}{l}\text { National population-based data on } \\
\text { disability and unmet health need }\end{array}$ & e & aa \\
\hline
\end{tabular}




\begin{tabular}{|c|c|c|c|c|c|c|}
\hline $\begin{array}{c}\text { Inter- } \\
\text { viewed } \\
(1997- \\
2000)\end{array}$ & $\begin{array}{c}\text { Classifi- } \\
\text { cation }\end{array}$ & Title & Author and year & Service area & $\begin{array}{l}\text { Objecti } \\
\text { ves }\end{array}$ & $\begin{array}{c}\text { Recom } \\
\text { mendati } \\
\text { ons }\end{array}$ \\
\hline • & 4 & $\begin{array}{l}\text { Aged Residential Care Utilisation in the Mid-North } \\
\text { Island }\end{array}$ & (Health Funding Authority, 2000a) & Aged Residential Care Utilisation & a & $\mathrm{bb}$ \\
\hline$\bullet$ & 1 & Improving our health in Wellington & (Health Funding Authority, 2000c) & $\begin{array}{l}\text { Health status of people of the greater } \\
\text { Wellington population and publicly funded } \\
\text { personal health services }\end{array}$ & $\mathrm{b}$ & gg \\
\hline \multirow[t]{3}{*}{$\bullet$} & 1 & $\begin{array}{l}\text { Report to the Ministry of Health: Health Profile of the } \\
\text { Wellington Region }\end{array}$ & $\begin{array}{l}\text { (HealthSearch, 1998) for Ministry of } \\
\text { Health }\end{array}$ & $\begin{array}{l}\text { Examines patterns of mortality and } \\
\text { hospitalisation in the Wellington region }\end{array}$ & e & gg \\
\hline & 1 & $\begin{array}{l}\text { A healthy future: report on Wairoa District health and } \\
\text { disability support services }\end{array}$ & $\begin{array}{l}\text { (Lane, 1994) Central Regional Health } \\
\text { Authority }\end{array}$ & $\begin{array}{l}\text { Organisation and delivery of community } \\
\text { health services, disabled and health } \\
\text { priorities }\end{array}$ & $\mathrm{b}$ & $\mathrm{cc}$ \\
\hline & 5 & Management Review of Tararua Health Services & $\begin{array}{l}\text { (Manawatu-Wanganui Area Health Board, } \\
\text { 1991) }\end{array}$ & $\begin{array}{l}\text { Health needs of the people of the Tararua } \\
\text { region, health services, and plans }\end{array}$ & $\mathrm{b}$ & $\mathrm{bb}$ \\
\hline \multirow[t]{2}{*}{$\bullet$} & 1 & Profile of the (Wellington) Locality & $\begin{array}{l}\text { (Maori Health Operating Group-Health } \\
\text { Funding Authority, 1999a, Maori Health } \\
\text { Operating Group-Health Funding } \\
\text { Authority, 1999b, Maori Health Operating } \\
\text { Group-Health Funding Authority, 1999c, } \\
\text { Maori Health Operating Group-Health } \\
\text { Funding Authority, 1999d, Maori Health } \\
\text { Operating Group-Health Funding } \\
\text { Authority, 1999e, Maori Health Operating } \\
\text { Group-Health Funding Authority, 1999f, } \\
\text { Maori Health Operating Group-Health } \\
\text { Funding Authority, 1999g, Maori Health } \\
\text { Operating Group-Health Funding } \\
\text { Authority, 1999h, Maori Health Operating } \\
\text { Group-Health Funding Authority, 1999i, } \\
\text { Maori Health Operating Group-Health } \\
\text { Funding Authority, 1999j, Maori Health } \\
\text { Operating Group-Health Funding } \\
\text { Authority, 1999k) }\end{array}$ & $\begin{array}{l}\text { Demographic profile, epidemiology and } \\
\text { health status of people in the locality }\end{array}$ & e & gg \\
\hline & 1 & Progress on Health Outcome Targets 1996 & (Ministry of Health, 1996) & $\begin{array}{l}\text { Epidemiological reports on progress } \\
\text { towards health outcome targets }\end{array}$ & c & aa \\
\hline • & 1 & $\begin{array}{l}\text { Making a Pacific difference: strategic initiatives for the } \\
\text { health of Pacific people in New Zealand }\end{array}$ & (Ministry of Health, 1997b) & $\begin{array}{l}\text { Ethnology and health need for Pacific } \\
\text { peoples in New Zealand }\end{array}$ & a & $\mathrm{ff}$ \\
\hline ○ & 5 & $\begin{array}{l}\text { Korero Pasifika: consultation review making a Pacific } \\
\text { difference }\end{array}$ & (Ministry of Health, 1997a) & $\begin{array}{l}\text { Community consultation workshop reports, } \\
\text { health status, consumer satisfaction, health }\end{array}$ & $\mathrm{f}$ & aa \\
\hline
\end{tabular}




\begin{tabular}{|c|c|c|c|c|c|c|}
\hline $\begin{array}{l}\text { Inter- } \\
\text { viewed } \\
(1997- \\
2000)\end{array}$ & $\begin{array}{l}\text { Classifi- } \\
\text { cation }\end{array}$ & Title & Author and year & Service area & $\begin{array}{l}\text { Objecti } \\
\text { ves }\end{array}$ & $\begin{array}{c}\text { Recom } \\
\text { mendati } \\
\text { ons }\end{array}$ \\
\hline & & & & planning and promotion, ethnology & & \\
\hline ○ & 1 & $\begin{array}{l}\text { Progress on health outcome targets, Te haere } \\
\text { whakamua ki nga whainga hua mo te hauora, } 1997\end{array}$ & (Ministry of Health, 1998b) & $\begin{array}{l}\text { Epidemiological reports on progress } \\
\text { towards health outcome targets }\end{array}$ & c & gg \\
\hline$\bullet$ & 1 & $\begin{array}{l}\text { Progress on health outcome targets, Te haere } \\
\text { whakamua ki nga whainga hua mo te hauora, } 1998\end{array}$ & (Ministry of Health, 1999c) & $\begin{array}{l}\text { Epidemiological reports on progress } \\
\text { towards health outcome targets }\end{array}$ & $\mathrm{c}$ & $\operatorname{gg}$ \\
\hline$\bullet$ & 1 & $\begin{array}{l}\text { Our children's health: key findings on the health of } \\
\text { New Zealand children }\end{array}$ & (Ministry of Health, 1998a) & Health needs of children & $\mathrm{c}$ & gg \\
\hline 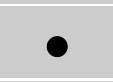 & 1 & $\begin{array}{l}\text { Our health our future = hauora pakari, koiora roa: the } \\
\text { health of New Zealanders, } 1999\end{array}$ & (Ministry of Health, 1999a) & $\begin{array}{l}\text { Mortality, morbidity, socioeconomic and } \\
\text { health status indicators for New Zealanders }\end{array}$ & c & gg \\
\hline \multirow[t]{3}{*}{$\bullet$} & 1 & $\begin{array}{l}\text { Taking the pulse: The 1996/97 New Zealand Health } \\
\text { Survey }\end{array}$ & (Ministry of Health, 1999d) & $\begin{array}{l}\text { National review of health status and health } \\
\text { service utilisation (extensive) }\end{array}$ & $\mathrm{c}$ & gg \\
\hline & 2 & Otara Community Primary and Public HNA & (Mitchell, 1995) for Ministry of Health & Community needs assessment & $\mathrm{b}$ & $\mathrm{bb}$ \\
\hline & 5 & $\begin{array}{l}\text { Disability support services: priorities: a Consensus } \\
\text { Development Conference report to the National } \\
\text { Advisory Committee on Core Health and Disability } \\
\text { Support Services }\end{array}$ & $\begin{array}{l}\text { (National Advisory Committee on Core } \\
\text { Health and Disability Support Services, } \\
\text { 1993) }\end{array}$ & $\begin{array}{l}\text { Disability support services and priorities }-\mathrm{a} \\
\text { consensus conference report }\end{array}$ & g & ee \\
\hline \multirow[t]{2}{*}{$\bullet$} & 5 & Review of maternity services in New Zealand & (National Health Committee, 1999) & $\begin{array}{l}\text { Review of needs, quality and standards of } \\
\text { maternal health services }\end{array}$ & e & $\mathrm{cc}$ \\
\hline & 5 & $\begin{array}{l}\text { Hauora Wahine Māori: A discussion document on } \\
\text { Māori women's health; Hauora Tane Māori: A } \\
\text { discussion document on Māori men's health }\end{array}$ & (North Health, 1996) & $\begin{array}{l}\text { Epidemiological data and discussion on } \\
\text { health issues for Māori women and men }\end{array}$ & d & gg \\
\hline$\bullet$ & 1 & Pacific Islands People in the North Health Region & (North Health, 1997) & $\begin{array}{l}\text { Population characteristics of Pacific people } \\
\text { in the North Health region }\end{array}$ & e & gg \\
\hline \multirow[t]{2}{*}{ • } & 1 & Socio-economic inequalities in health care & (North Health, 1998) & $\begin{array}{l}\text { Epidemiological analysis of health and } \\
\text { socio-economic analysis }\end{array}$ & c & gg \\
\hline & 1 & Māori in the North Health Region & (Northern Regional Health Authority, 1995) & Demographic and health status analysis & e & gg \\
\hline$\bullet$ & 1 & The Northern Region Health Survey: 1996/97 & $\begin{array}{l}\text { (Parr, Whittaker and Jackson, 1998) for } \\
\text { North Health }\end{array}$ & Health status and need survey & e & gg \\
\hline$\bullet$ & 2 & Case study: Maternity Services and Care in Porirua & $\begin{array}{l}\text { (Porirua Community Health Group and } \\
\text { Porirua Health Partnership, 1997) for } \\
\text { Central Regional Health Authority }\end{array}$ & Maternity service need in Porirua & g & $\mathrm{bb}$ \\
\hline$\bullet$ & 1 & Kapiti District Health and Disability Report and Plan & $\begin{array}{l}\text { (Porirua Kapiti Healthlinks Project, 2000a) } \\
\text { for Ministry of Health }\end{array}$ & All health services & $\mathrm{b}$ & dd \\
\hline$\bullet$ & 1 & Porirua District Health and Disability Report and Plan & $\begin{array}{l}\text { (Porirua Kapiti Healthlinks Project, 2000b) } \\
\text { for Ministry of Health }\end{array}$ & All health services & $\mathrm{b}$ & dd \\
\hline
\end{tabular}


Appendix Three

\begin{tabular}{|c|c|c|c|c|c|c|}
\hline \multirow[t]{9}{*}{$\begin{array}{c}\text { Inter- } \\
\text { viewed } \\
(1997- \\
2000)\end{array}$} & $\begin{array}{l}\text { Classifi- } \\
\text { cation }\end{array}$ & Title & Author and year & Service area & $\begin{array}{l}\text { Objecti } \\
\text { ves }\end{array}$ & $\begin{array}{c}\text { Recom } \\
\text { mendati } \\
\text { ons }\end{array}$ \\
\hline & 1 & $\begin{array}{l}\text { Our health, our future = hauora pakari, koiora roa: The } \\
\text { State of the public health in New Zealand } 1993\end{array}$ & (Public Health Commission, 1993) & $\begin{array}{l}\text { New Zealand epidemiological data - major } \\
\text { resource }\end{array}$ & $\mathrm{c}$ & gg \\
\hline & 3 & $\begin{array}{l}\text { Sudden Infant Death Syndrome (SIDS): The Public } \\
\text { Health Commission's Advice to the Minister of Health } \\
\text { 1993-1994 }\end{array}$ & (Public Health Commission, 1994d) & Review of sudden infant death syndrome & $\mathrm{e}$ & $\mathrm{cc}$ \\
\hline & 3 & $\begin{array}{l}\text { Alcohol: The Public Health Commission's Advice to } \\
\text { the Minister of Health 1993-1994 }\end{array}$ & (Public Health Commission, 1994a) & $\begin{array}{l}\text { Review of alcohol prevention and treatment } \\
\text { needs }\end{array}$ & e & dd \\
\hline & 1 & $\begin{array}{l}\text { Our health, our future = hauora pakari, koiora roa: The } \\
\text { State of the public health in New Zealand } 1994\end{array}$ & (Public Health Commission, 1994c) & $\begin{array}{l}\text { New Zealand epidemiological data - major } \\
\text { resource }\end{array}$ & $\mathrm{c}$ & gg \\
\hline & 1 & Whakapiki mauri = Māori health advancement & (Public Health Commission, 1995) & $\begin{array}{l}\text { Health status indicators for Māori, public } \\
\text { health and socioeconomic factors }\end{array}$ & c & gg \\
\hline & 1 & The health of Pacific Islands people in New Zealand & (Public Health Commission, 1994b) & $\begin{array}{l}\text { Ethnology, health status and socioeconomic } \\
\text { factors for Pacific peoples }\end{array}$ & $\mathrm{c}$ & gg \\
\hline & 1 & $\begin{array}{l}\text { Whangarei area health and disability support services } \\
\text { needs assessment }\end{array}$ & (Scanlen, 1995) for North Health & $\begin{array}{l}\text { Epidemiological, health services and } \\
\text { community consultation regarding health } \\
\text { need assessment in Whangarei area }\end{array}$ & $\mathrm{b}$ & $\mathrm{bb}$ \\
\hline & 4 & $\begin{array}{l}\text { Primary mental health care: a discussion paper on } \\
\text { current issues and service provision }\end{array}$ & (Simpson, 1993) for Department of Health & $\begin{array}{l}\text { Community mental health service needs } \\
\text { and demands }\end{array}$ & $\mathrm{a}$ & ff \\
\hline
\end{tabular}




\section{Appendix 4: HNA Ethics Approval}

\section{Gregor Coster}

From: Maggie Teleki-Rainey<maggie.teleki-rainey@vuw.ac.nz>

To: $\quad<$ g.coster@auckland.ac.nz>

Cc: Claudia Scott <claudia.scott@vuw.ac.nz>

Sent: $\quad$ Saturday, 14 July 2001 11:27

Subject: Human Ethics Application - Approval

\section{To : Gregor Coster}

On behalf of : Gordon Anderson, Associate Dean, Graduate Studies and Research

Please note that the Human Ethics Committee has reviewed and approved your application

for the project "The Usefulness of Health Needs Assessment in influencing heath service delivery and policy: the last decade".

Regards.

Maggie.

cc : Linda Bowden, Vice-Chancellor's Office

Maggie Teleki-Rainey

Administration Assistant to the Associate Deans

Faculty of Commerce and Administration

Victoria University of Wellington

Murphy Building - MY 314

PO Box 600

Wellington

Phone : (04) 4635943

Fax : (04) 4635360

E-Mail : Maggie.Teleki-Rainey@vuw.ac.nz 


\section{Appendix 5: Invitation letter to 1997-2000 interview participants}

Professor Gregor Coster

105 Ngapuhi Road

Remuera

Auckland 1005

DATE

\section{NAME \& ADDRESS}

Dear xxx

\section{Re: Telephone interview}

I am presently undertaking a Ph.D. degree in Public Policy the Victoria University of Wellington. I am conducting some research on the usefulness of HNAs in influencing health service delivery and policy over the last decade. The HNAs that I am particularly interested in presently are those that were conducted and published during the last four years, in the public sector. I think that there are some lessons to be learned and some benefits to gain in studying these HNAs, prior to evaluating the HNA processes of District Health Boards.

I am planning to interview two people associated with each identified HNA conforming to the inclusion criteria of the study. In most cases, this will involve a person who was responsible for, or who had a major role to play in the project, and a manager who may have commissioned the project.

During the course of researching, I have found the following project(s), which meet the criteria, in which you appear to have to have had a significant role:

INSERT PROJECT NAME(s) 
I am writing to ask if you would agree to be interviewed about this project. I would like to ascertain your views in regard to the assessment and its overall effectiveness in achieving its objectives. Interviews will take approximately 20 minutes by telephone or in person, and if you give your consent I would like to audiotape the interview. I enclose an information sheet regarding the research and a consent form for signing. I have received ethics approval from the Victoria University of Wellington Human Ethics Committee to satisfy the requirements of privacy and other ethical considerations. This is a prerequisite for interviewing anyone for a project under the University's name.

I would be most grateful if you could contact me at the above address, or call me on (09) 373-7599 ex 6518, Mob 021998265 or fax me on (09) 367-7131. My email address is g.coster@auckland.ac.nz. Thank you very much for taking the time to read this letter. I look forward to the possibility of interviewing you.

Kind regards

Yours sincerely

Gregor Coster

My supervisors are:

Nicholas Mays

Social Policy Branch

Treasury

1 The Terrace

Wellington

Tel (04) 4715162
Prof Claudia Scott

Head of Department

Department of Public Policy

Victoria University of Wellington

PO Box 600, Wellington

Tel (04) 4721000

For any queries regarding ethical concerns please contact Prof Gordon Anderson, Associate Dean, Graduate Studies and Research, Victoria University of Wellington. 


\section{Appendix 6: Information Sheet (Past Interviews 1997-2000)}

Participant Information Sheet for a study on HNA.

I am undertaking a $\mathrm{PhD}$ at the Victoria University of Wellington. As part of this research, I am studying the usefulness of HNAs in influencing health service delivery and policy, particularly over the last four years.

I am planning to interview two people associated with each identified HNA conforming to the inclusion criteria of the study. In most cases, this will involve a person who was responsible for, or who had a major role to play in the project, and a manager who may have commissioned the project.

Participants will be asked a number of questions, using a semi-structured interview format, regarding how needs assessments entered on the policy agenda, the objectives, the actions that resulted and an assessment of their effect in influencing purchasing decisions. Estimates of the cost of studies will also be sought.

Interviews will generally be conducted as taped telephone interviews, although if the opportunity offers they may be conducted as taped face to face interviews. It is anticipated that interviews will take 20-25 minutes.

Should participants feel the need to withdraw from the project, they may do so at any time without question. Just let me know at the time.

Responses collected by tape will be listened to and analysed into groups of responses. The analysis of the responses will form the basis of my report. Only grouped responses will be presented in the report and it will not be possible for you to be identified 
personally. My supervisors will have access to all data including names. The data will otherwise remain confidential.

This work will form part of a thesis to be submitted for assessment at the Victoria University of Wellington. It will be deposited in the University Library. It is possible that a paper will be submitted to a peer-reviewed journal. Tapes and notes will be destroyed at the end of the project.

If you have any questions or would like to receive further information please contact me on (09) 3737599 (ext 6518) or my supervisor, Prof Claudia Scott on (04) 472 1000, or at Victoria University, PO Box 600, Wellington.

Gregor Coster 


\section{Appendix 7: Consent form (Past Interviews 1997-2000) \\ VICTORIA UNIVERSITY OF WELLINGTON \\ CONSENT TO PARTICIPATION IN RESEARCH}

Title of project: The usefulness of HNAs in influencing health service delivery and policy: the last decade.

I have been given and have understood an explanation of this research project. I have had an opportunity to ask questions and have them answered to my satisfaction. I understand that I may withdraw myself (or any information I have provided) from this project (before data collection and analysis are complete) without having to give reasons or without penalty of any sort.

I understand that any information that I provide will be kept confidential to the researcher and supervisors. The published results will not use my name, and that no opinions will be attributed to me in any way that will identify me. I understand that the tape recording of interviews will be electronically wiped at the end of the project unless I indicate that I would like them returned to me.

I would like the tape recordings of my interview returned to me at the conclusion of the project.

I understand that the data I provide will not be used for any other purpose or released to others without my written consent. 
I would like to receive a summary of the results of this research when it is completed.

I agree to take part in this research.

Signed:

Name of participant

(please print clearly)

Date: 


\section{Appendix 8: Semi-structured interview guide for interviews with} HNA participants (1997-2000)

\begin{tabular}{|c|c|}
\hline Questions & Probes \\
\hline $\begin{array}{l}\text { 1. How many HNAs did your organisation conduct between } 1997- \\
2000 \text { ? }\end{array}$ & If more than one: What were these? \\
\hline 2. What was your involvement or role in the ....(HNA of interest)? & $\begin{array}{l}\text { Manager? } \\
\text { Public health physician? } \\
\text { What was your contribution? }\end{array}$ \\
\hline 3. Why was this project undertaken? & $\begin{array}{l}\text { Statutory requirement? } \\
\text { Commissioned work? Annual publication? } \\
\text { Follow up on some national policy? } \\
\text { Pressure exerted on the sponsoring body? } \\
\text { Regional initiative? Local concern? Some evidence of a } \\
\text { problem? } \\
\text { Special pressure or interest from an individual within the } \\
\text { health authority? } \\
\text { Routinely collected data? } \\
\text { Assist a purchasing decision? } \\
\text { Purely for research purposes? Any other reasons? }\end{array}$ \\
\hline \multicolumn{2}{|l|}{ 4. How did your organisation undertake this project? } \\
\hline $\begin{array}{l}\text { 5. From your perspective, what were the key outcomes of this } \\
\text { project? }\end{array}$ & $\begin{array}{l}\text { Has there been any improvement in health outcomes? What is } \\
\text { the evidence? }\end{array}$ \\
\hline 6. What actions resulted from this project? & $\begin{array}{l}\text { Were there any qualitative changes to existing services? } \\
\text { Quantitative changes? } \\
\text { Was it a service review resulting in no change to the service? } \\
\text { Any other research required? } \\
\text { What other actions were taken as a result of the needs } \\
\text { assessment? } \\
\text { Were the recommendations of the report implemented? } \\
\text { If so, how successfully? If not, what were the barriers? }\end{array}$ \\
\hline 7. To what extent did the project influence purchasing decisions? & $\begin{array}{l}\text { How useful was it? } \\
\text { Did it add anything to the previous knowledge? } \\
\text { Was the exercise just used as a management tool? } \\
\text { Had the decision to fund particular services already been } \\
\text { made? } \\
\text { Did government policy settings around priority objectives } \\
\text { determine the outcome anyway? } \\
\text { Do you thing that the needs of low socio-economic groups } \\
\text { were allowed for? } \\
\text { Did Māori have a say? }\end{array}$ \\
\hline \multicolumn{2}{|l|}{ 8. To what extent were health needs prioritised? } \\
\hline \multicolumn{2}{|l|}{ 9. What lessons can be learnt from this HNA exercise? } \\
\hline $\begin{array}{l}\text { 10. Is there anything further on which you would like to comment or } \\
\text { elaborate? }\end{array}$ & Usefulness of HNAs? \\
\hline $\begin{array}{l}\text { 11. What are the three most important points you would like me to } \\
\text { take away from this interview? }\end{array}$ & \\
\hline
\end{tabular}




\title{
Appendix 9: Invitation letter to Planning and Funding Managers
}

\author{
General Practice \& Primary Health Care \\ Te Tari Tawaiora
}

General Manager Planning,

Purchasing and Population Health

Nelson Marlborough DHB

Dear

\section{Re: Health Reforms Research - Interview}

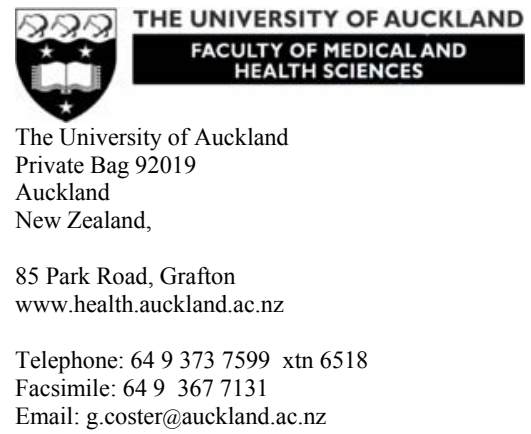

You may be aware that a national team of researchers has been funded to research the current health reforms and their implementation. The aims of the research are to analyse the new governance, purchasing and accountability arrangements which develop under the District Health Board model. Ms Jackie Cumming, Principal Investigator, has already sent a letter to the Chair and CEO of your Board in regard to the research, a copy of which is attached.

As part of the study, the research team is studying the HNA and prioritisation process in the context of the strategic planning cycle. I am writing to seek your agreement for an hour long interview (it may not take an hour) in your role as General Manager Planning, or equivalent. The interview will be conducted by telephone and with your permission will be taped and later transcribed, with the transcript returned to you for checking for accuracy. As noted in the attached letter to Chairs and CEOs, we will ensure the confidentiality of interviews, documents, and of individual interviewees.

We hope that the research will identify innovative practices that we can share with other boards, and we intend to release general information to you as the research progresses. 
Reporting of the results will focus on general themes, drawn from the information we gain from all DHBs, and from the case studies.

I hope that you are agreeable to participating in this research, and in anticipation, I will ask my PA, Diane Nicholson, to contact you in the next few days to arrange a suitable time for interview. For your interest, I enclose a copy of the questions that will be asked at the interview.

I hope that you will be willing to participate and look forward to our interview. My contact details are:

Phone: 093737599 Xtn 6518

Fax: $\quad 093677131$

Email: g.coster@auckland.ac.nz

Yours sincerely

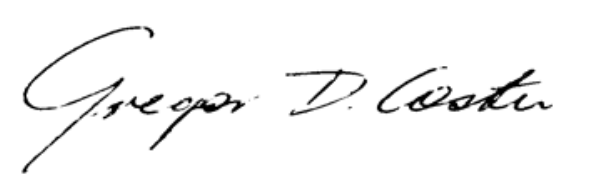

Gregor D Coster

Elaine Gurr Professor of General Practice 


\section{Appendix 10: Information Letter for Planning and Funding Managers}

11 April 2002

«Title» «FirstName» «LastName»

«JobTitle»

«Company»

«Address1»

«Address2»

«City»

cc. «DHBceo»

cc. Mr Julian Inch, DHB NZ

Dear «FirstName»

\section{Re: Health Reforms Research}

As you may be aware, a national team of researchers has been funded to research the current health reforms and their implementation, over the next few years. The research was discussed at the DHB NZ Board meeting in December 2001, and has the broad support of the Board.

The aims of the research are to analyse the new governance, purchasing and accountability relationships which develop under the District Health Board (DHB) model, and to assess the strength and weaknesses of the model compared with alternative models of health care organisation, principally the market model established in the later 1990s. The research is also focusing on the establishment of DHBs, the implementation of the various national Strategies, and capacity in the sector to manage health care under the new model. An information sheet on the research is attached.

I am writing to you to provide information on the project and to seek the involvement of your DHB in the research. The ways in which your DHB might be involved are set out below. 
Phases of the research

The research involves four phases. A first phase is to establish the objectives of the reforms, and experience with establishing the model to date. This involves a number of activities:

We are currently undertaking a series of key informant interviews with Ministers of the Crown and key government officials about their expectations of the reforms and experience to date. We will also undertake questionnaires or interviews with other key stakeholder organisations, such as IPAs, DHBNZ, HealthCare Aotearoa, Māori/iwi, non-government organisations, and professional organisations in May/June 2002 on similar themes.

We are aiming to interview by phone all Chief Executive Officers (CEOs) of DHBs, in May/June 2002; focusing on expectations and experiences to date of the reforms.

A questionnaire will be sent out to all board members of DHBs in May 2002.

Planning and Funding Managers will also be interviewed in May/June 2002 in relation to priority setting processes undertaken in the development of strategic and annual plans.

We will interview Chairs of DHBs later in 2002.

This research process will be repeated again in early 2004 in order to see how implementation has progressed.

A second phase of the research involves the collection of documentation on the reforms, including central government policies and statements, strategies, performance management documentation, annual plans, etc. Much of this material is available publicly, including on your websites, and through other public sources, but we are likely to seek other information from your board.

A third phase of the research involves looking at a number of other issues, such as the performance of the Ministry of Health in supporting the reformed sector, identifying the 
public's perception of the reforms, and seeing what implications the reforms have for the wider public sector. Many of these issues will be researched using the methods described above.

\section{Case studies of five districts}

Phases one to three will consider a range of issues across all DHBs, and involve a range of key stakeholders. A fourth phase of the research is to focus on a number of case study districts. Here the research will especially look at decision making (including priority setting), governance issues (i.e., the functioning of the board, accountability to local populations and to central government, and including contracting with providers), and Māori and Pacific health progress. This phase will involve a number of key informant interviews with senior DHB staff, including hospital managers; with a range of providers with whom DHBs contract for services; and with other key stakeholders in each district. It will also involve document analysis (e.g., minutes of meetings, strategies, annual plans, and internal documentation where available), and observation of board meetings and any other meetings your DHB advises as useful for this research. This will commence in mid-late 2002; and will also be repeated in early 2004.

We are writing to five DHBs in the districts we wish to involve as case studies, seeking their participation in the research. These DHBs are Waitemata, Tairawhiti, Manawatu, Capital and Coast Health and Canterbury DHBs. They have been chosen because they are of interest for the following reasons; that is they, a) represent both small (Tairawhiti), medium (Manawatu) and large (Capital and Coast, Waitemata, Canterbury) DHBs in terms of population size; b) cover DHBs which have a sizeable percentage of the population which is Māori (Capital and Coast, Waitemata, Manawatu, and in particular Tairawhiti); c) cover rural and provincial (Tairawhiti, Manawatu, Canterbury), and urban districts (Capital and Coast, Waitemata, Canterbury); d) are reasonably accessible to the research team (Capital and Coast, Waitemata, Manawatu, Canterbury), and e) are those districts where some of the research team members have key contacts and good working relationships. 
Involving your $D H B$ in the research

All DHBs will be involved in some phases of the research, and we hope that you will participate in phone interviews, and encourage board members to respond to our questionnaire which will be sent out in May. We will be phoning CEOs before the end of April to arrange interview times with respect to the reforms implementation process to date.

Your district is one which we would like to include as a case study district. As noted above, this will involve intensive research in your district, including interviewing key personnel. «Researcher», a member of the research team, will contact you soon to explain the proposed case studies in more detail and to invite you to participate.

\section{Confidentiality and ethics}

In discussing your role in the district case studies, we aim to work through issues relating to access to documentation and confidentiality. It won't be possible for us to keep secret the identity of the case study districts, but we always ensure confidentiality of documents, and of individual interviewees. We hope that the research will identify innovative practices that we can share these with other boards, so we aim to work through the release of specific information on your board's activities with you as the research progresses.

Our reporting of the results of the research will focus on general themes, drawn from both the information we gain from all DHBs and from the case studies. We will always provide drafts of material to you prior to its release.

We are currently discussing the different phases of the research with the Wellington Ethics Committee and will work with them on all ethical issues. 


\section{Formative approach}

A key aspect of the research is its formative approach, where the researchers will be feeding back findings to interested parties as we progress. It is our intention for the research to enable all stakeholders to learn from the general experiences we identify, and for these results to be fed back to the sector in a timely fashion. This will enable early identification of issues for discussion within and between DHBs and other stakeholders, and with the Ministry of Health, Treasury and State Services Commission. As noted above, we hope that the research will identify innovative practices that can be shared with all DHBs and other stakeholders. We hope that all DHBs will see some value in this for them over the next few years.

\section{Independence}

We reiterate the independence of the research team from any government agencies. We also note that any assessment of performance will relate only to the broad DHB model of health service delivery for New Zealand, not to the performance of individual DHBs.

Although Ministry of Health staff are involved in the research for evaluation training purposes (see Research Information Sheet), they will be responsible to me as the Principal Investigator, they will sign confidentiality agreements, and they will not pass information between this project and performance management teams at the Ministry of Health.

We look forward to working with you on this exciting project and will be in touch very shortly.

Yours sincerely

Jackie Cumming

Principal Investigator

Health Reforms Research Team 


\section{Appendix 11: Consent form for Planning and Funding Manager Interviews}

\section{CONSENT FORM}

Health reforms 2001: Assessing governance, purchasing and accountability in the New Zealand health sector.

I agree to be interviewed for this research project .

口 I agree to the tape-recording of the interview, understanding that copies of the tapes will be kept in a locked cabinet, and erased 3 years after the completion of the research.

口 I wish to have a transcript of the interview sent to me for checking, and I will return the corrected transcript to the Research Team within four weeks of receiving it.

口 I understand that tapes will be heard and transcripts will be read only by the Research Team.

口 I understand that I may withdraw information from the Project at any time.

Signed: 
Name:

Date:

Return to:

Professor Gregor Coster

Department of General Practice \& Primary Health Care

Private Bag 92019

Auckland

Fax: 093677131

(This Consent Form was the standard form used by the Health Reforms 2001 Research Group for this research project.) 


\section{Appendix 12: Semi-structured interview guide for interviews with DHB funding and planning managers}

\begin{tabular}{|c|c|}
\hline Questions & Probes \\
\hline \multicolumn{2}{|l|}{ What was your particular role in the HNA and priority setting process? } \\
\hline What difficulties did the DHB find in conducting the HNA? & $\begin{array}{l}\text { Workplan timeframe } \\
\text { High workload and stress? } \\
\text { Capacity issues? } \\
\text { Resources? } \\
\text { Access to data? } \\
\text { Data standardisation? }\end{array}$ \\
\hline Did your Board use an external contractor for the HNA? & $\begin{array}{l}\text { If WCS: how responsive to local need do } \\
\text { you think that your HNA was? }\end{array}$ \\
\hline \multicolumn{2}{|l|}{ What do you estimate the cost to your Board for the HNA exercise? } \\
\hline \multicolumn{2}{|l|}{ What would you do differently if you did the HNA again? } \\
\hline \multicolumn{2}{|l|}{ Did you consult with the community during the HNA? } \\
\hline \multicolumn{2}{|l|}{ What level of participation by Māori was there in the HNA? } \\
\hline \multicolumn{2}{|l|}{ What level of participation did Pacific have in the HNA? } \\
\hline \multicolumn{2}{|l|}{$\begin{array}{l}\text { Did your HNA throw up a single key issue that was helpful in your } \\
\text { planning process? }\end{array}$} \\
\hline \multicolumn{2}{|l|}{ What gaps did you find in the data? } \\
\hline Does your Board plan to do any other HNAs? & $\begin{array}{l}\text { e.g. to review progress? } \\
\text { Disability Support Services (e.g. WCS } \\
\text { process?) }\end{array}$ \\
\hline \multicolumn{2}{|l|}{$\begin{array}{l}\text { To what extent is it possible for the DHB to make resource allocation needs } \\
\text { based on local needs and values? }\end{array}$} \\
\hline \multicolumn{2}{|l|}{$\begin{array}{l}\text { Can you make an estimate of the percentage of resources that are already } \\
\text { committed on a year to year basis? }\end{array}$} \\
\hline $\begin{array}{l}\text { Which services received significant increases in funding? (in the Annual } \\
\text { Plan/DSP)? }\end{array}$ & $\begin{array}{l}\text { New services? } \\
\text { Justification of increase (i.e. reference to } \\
\text { explicit principles or 'on it own merits') } \\
\text { Identify budget lines? }\end{array}$ \\
\hline Which services had their funding cut significantly? & $\begin{array}{l}\text { Any disinvestments? (in the Annual } \\
\text { Plan/DSP) } \\
\text { Justification of decreases (i.e. reference to } \\
\text { explicit principles or 'on its own merits') } \\
\text { Identify budget lines? }\end{array}$ \\
\hline $\begin{array}{l}\text { What factors were most influential in shaping the coming years allocation } \\
\text { of resources? }\end{array}$ & $\begin{array}{l}\text { Historical factors? } \\
\text { HNA process? } \\
\text { Government strategies? } \\
\text { Stakeholder pressures? } \\
\text { Community input? } \\
\text { Cost-utility analysis? } \\
\text { Māori health need? }\end{array}$ \\
\hline
\end{tabular}




\begin{tabular}{|c|c|}
\hline $\begin{array}{l}\text { What is the process for deciding resource allocation? (referring here to } \\
\text { prioritisation framework) }\end{array}$ & $\begin{array}{l}\text { by principles/values established a priori (if } \\
\text { so, what are they?) } \\
\text { by principles/values established during the } \\
\text { process } \\
\text { no guiding principles/values, decisions } \\
\text { made on a case-by-case basis. } \\
\text { For a) and b), were these principles } \\
\text { related to the HFA criteria? (i.e. } \\
\text { effectiveness, cost, equity, Máori needs } \\
\text { and acceptability). }\end{array}$ \\
\hline \multicolumn{2}{|l|}{$\begin{array}{l}\text { Was there Māori participation in the assessment of competing claims (or the } \\
\text { establishment of priorities), and if so, what form did it take? }\end{array}$} \\
\hline \multicolumn{2}{|l|}{$\begin{array}{l}\text { What priority is being given to Pacific needs in terms of allocation of } \\
\text { resources/funding? }\end{array}$} \\
\hline $\begin{array}{l}\text { Thinking about the DSP, what were the factors affecting the direction the } \\
\text { Plan took? }\end{array}$ & Any other influences? \\
\hline How did you consult with the community on the DSP? & How many meetings? \\
\hline \multicolumn{2}{|l|}{ How was community feedback incorporated into the Plan? } \\
\hline \multicolumn{2}{|l|}{ Did you write your DAP before the DSP? } \\
\hline Did you find your HNA a very useful process? & $\begin{array}{l}\text { Living document? } \\
\text { Allowed influence by low socio-economic } \\
\text { groups, either directly or indirectly? } \\
\text { Allowed influence by Māori? }\end{array}$ \\
\hline Are there any key things that can be done to improve the use of the HNA? & Intersectoral partnerships? \\
\hline $\begin{array}{l}\text { Overall, thinking about the HNA process, how well joined-up are the } \\
\text { following? }\end{array}$ & $\begin{array}{l}\text { HNA and prioritisation? } \\
\text { Prioritisation and DSP? } \\
\text { DAP and DSP? } \\
\text { DAP and budget? } \\
\text { Budget and purchasing? }\end{array}$ \\
\hline $\begin{array}{l}\text { Finally, have you got any other general comments that you would like to } \\
\text { make? }\end{array}$ & \\
\hline
\end{tabular}




\section{Appendix 13: DHB needs assessment recommendations, and}

\section{priorities in district strategic and annual plans}

Nelson Marlborough DHB

HNA Key recommendations Intersectoral action

Smoking

Alcohol \& drug

Maori health

Health informantion

HIth status low socio-economic

Primary care

Avoidable admissions

Rural health

Nutrition

Physical activity

Diabetes

Oral health

Mental health

Disability support

Integrated services

Child health

Motor vehicle injuries

Immunisation rates

Violence

Water quality

Air quality

\section{Bay of Plenty DHB}

HNA Key recommendations Maori health

Unintentional injuries

Motor vehicle injuries

Suicide

Violence

Cancer

Cardiovascular disease

Diabetes

Sexually transmitted disease

Teenage fertility

Respiratory disease

Digestive diseases

Musculoskeletal diseases

Oral health

HIth of low socio-economic

Smoking

Nutrition

Alcohol \& drug

Falls >65yrs

SIDS

Mental health

Child health

Immunisation

Obesity

Physical activity

\begin{tabular}{|c|c|c|}
\hline Prioritisation & Level of importance in DSP & Level of importance in DAP \\
\hline $1=$ Key priority & 1=Performance measure & 1=Performance measure \\
\hline 2=Significant priority & 2=Planned initiative (defined) & 2=Planned initiative (defined) \\
\hline 3=Less important prior & 3=Planned (not defined) & 3=Planned (not defined) \\
\hline 4=Not mentioned & 4=Not mentioned & $4=$ Not mentioned \\
\hline 1 & 1 & 1 \\
\hline 1 & 1 & 1 \\
\hline 3 & 3 & 2 \\
\hline 1 & 1 & 1 \\
\hline 3 & 2 & 1 \\
\hline 1 & 1 & 1 \\
\hline 2 & 3 & 1 \\
\hline 3 & 3 & 3 \\
\hline 2 & 3 & 2 \\
\hline 1 & 1 & 1 \\
\hline 1 & 1 & 1 \\
\hline 1 & 1 & 1 \\
\hline 1 & 1 & 1 \\
\hline 1 & 1 & 1 \\
\hline 1 & 2 & 1 \\
\hline$\overline{1}$ & 1 & $\overline{1}$ \\
\hline 3 & 3 & 2 \\
\hline 3 & 4 & 4 \\
\hline 1 & 3 & 1 \\
\hline 3 & 4 & 3 \\
\hline 4 & 3 & 4 \\
\hline 4 & 4 & 4 \\
\hline
\end{tabular}

Prioritisation

Level of importance in DSP Level of importance in DAP

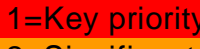

$\begin{array}{ll}\text { 1=Performance measure } & \text { 1=Performance measure } \\ \text { 2=Planned initiative (defined) } & \text { 2=Planned initiative (defined) }\end{array}$

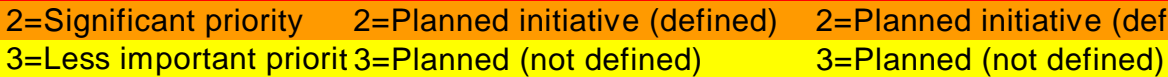

$4=$ Not mentioned $\quad 4=$ Not mentioned $\quad 4=$ Not mentioned

\begin{tabular}{|c|c|c|}
\hline 4=Not mentioned & 4=Not mentioned & 4=Not mentioned \\
\hline 1 & 1 & 1 \\
\hline 3 & 3 & 3 \\
\hline 3 & 4 & 4 \\
\hline 1 & 3 & 1 \\
\hline 1 & 4 & 3 \\
\hline 1 & 4 & 3 \\
\hline 2 & 4 & 4 \\
\hline 1 & 4 & 1 \\
\hline 2 & 4 & 4 \\
\hline 2 & 4 & 4 \\
\hline 2 & 4 & 1 \\
\hline 2 & 4 & 4 \\
\hline 2 & 4 & 4 \\
\hline 1 & 4 & 1 \\
\hline 1 & 3 & 3 \\
\hline 1 & 4 & 4 \\
\hline 1 & 4 & 4 \\
\hline 2 & 3 & 4 \\
\hline 3 & 4 & 4 \\
\hline 3 & 4 & 4 \\
\hline 2 & 3 & 1 \\
\hline 1 & 1 & 1 \\
\hline 1 & 4 & 1 \\
\hline 1 & 4 & 4 \\
\hline 1 & 3 & 4 \\
\hline
\end{tabular}




Taranaki DHB
HNA Key recommendations
Maori health
Children's hearing
Intersectoral action
Smoking
Alcohol \& drugs
Workforce development
Primary care
Rural health
Primary /secondary interface
Public health
Health information
Diabetes
Cancer
Cardiovascular disease
Respiratory disease
Suicide
Nutrition/Obesity/Inactivity
Mental health
Motor vehicle injury
Teenage pregnancy
Sexually transmitted disease
Violence
Cervical \& breast screening
Falls $>65 y e a r s$

\section{Lakes DHB}

HNA Key recommendations

Maori health

Expected baseline services

Deprivation

Smoking

Nutrition/obesity

Physical activity

Suicide

Alcohol \& drug

Cancer

Cardiovascular disease

Diabetes

Oral health

Violence

Child health

Mental health

Primary care

Motor vehicle injuries

Unintentional injury

Workforce development

Teenage pregnancy

Perinatal mortality

Water quality

Intersectoral action

Health information

Communicable diseases

\begin{tabular}{|c|c|c|}
\hline Prioritisation & Level of importance in DSP & Level of importance in DAP \\
\hline 1=Key priority & 1=Performance measure & 1=Performance measure \\
\hline 2=Significant priority & 2=Planned initiative (defined) & 2=Planned initiative (defined) \\
\hline 3=Less important prior & t 3=Planned (not defined) & 3=Planned (not defined) \\
\hline 4=Not mentioned & 4=Not mentioned & 4=Not mentioned \\
\hline 2 & 2 & 1 \\
\hline 2 & 2 & 1 \\
\hline 2 & 3 & 3 \\
\hline 1 & 2 & 2 \\
\hline 2 & 3 & 2 \\
\hline 1 & 2 & 1 \\
\hline 2 & 3 & 1 \\
\hline 2 & 3 & 3 \\
\hline 2 & 3 & 1 \\
\hline 2 & 3 & 3 \\
\hline 2 & 2 & 2 \\
\hline 2 & 3 & 1 \\
\hline 2 & 3 & 3 \\
\hline 2 & 3 & 3 \\
\hline 2 & 3 & 1 \\
\hline 2 & 3 & 1 \\
\hline 1 & 2 & 2 \\
\hline 2 & 3 & 1 \\
\hline 2 & 4 & 4 \\
\hline 2 & 4 & 4 \\
\hline 2 & 4 & 4 \\
\hline 2 & 3 & 3 \\
\hline 2 & 3 & 1 \\
\hline 2 & 3 & 3 \\
\hline
\end{tabular}

\begin{tabular}{|c|c|c|}
\hline Prioritisation & Level of importance in DSP & Level of importance in DAP \\
\hline 1=Key priority & 1=Performance measure & 1=Performance measure \\
\hline 2=Significant priority & 2=Planned initiative (defined) & 2=Planned initiative (defined) \\
\hline 3=Less important priori & t 3=Planned (not defined) & 3=Planned (not defined) \\
\hline 4=Not mentioned & 4=Not mentioned & 4=Not mentioned \\
\hline 1 & 1 & 1 \\
\hline 1 & 1 & 1 \\
\hline 1 & 1 & 1 \\
\hline 2 & 2 & 1 \\
\hline 2 & 2 & 2 \\
\hline 2 & 2 & 3 \\
\hline 2 & 3 & 2 \\
\hline 2 & 2 & 1 \\
\hline 2 & 2 & 2 \\
\hline 2 & 2 & 3 \\
\hline 2 & 2 & 1 \\
\hline 2 & 2 & 1 \\
\hline 2 & 4 & 2 \\
\hline 2 & 2 & 1 \\
\hline 2 & 2 & 1 \\
\hline 2 & 2 & 1 \\
\hline 3 & 3 & 1 \\
\hline 2 & 4 & 1 \\
\hline 3 & 2 & 2 \\
\hline 3 & 2 & 3 \\
\hline 3 & 2 & 1 \\
\hline 3 & 3 & 1 \\
\hline 2 & 3 & 1 \\
\hline 3 & 2 & 1 \\
\hline 3 & 2 & 2 \\
\hline
\end{tabular}


Wairarapa DHB

HNA Key recommendations

Low socio-economic status

Maori health

Smoking

Nutrition

Obesity

Physical activity

Suicide

Alcohol \& drugs

Cancer

Cardiovascular disease

Diabetes

Oral health

Violence

Mental health

Child health

Asthma

Childrens unintentional injuries Teenage pregnancy rates Pregnancy related complication Avoidable hospitalisations Maori post-natal mortality Immunisation rates

Workforce development

Health information

Primary health care

Primary/secondary integration Intersectoral action

\section{South Canterbury DHB}

\section{HNA Key recommendations}

Low socio-economic status

Maori health

Cardiovascular disease

Diabetes

Smoking

Oral health

Rural health

Prescribing costs

Maori provider development Motor vehicle injuries

\begin{tabular}{|c|c|c|}
\hline Prioritisation & Level of importance in DSP & Level of importance in DAP \\
\hline 1=Key priority & 1=Performance measure & 1=Performance measure \\
\hline 2=Significant priority & 2=Planned initiative (defined) & 2=Planned initiative (defined) \\
\hline 3=Less important priol & 3=Planned (not defined) & 3=Planned (not defined) \\
\hline 4=Not mentioned & 4=Not mentioned & 4=Not mentioned \\
\hline 2 & 3 & 2 \\
\hline 2 & 2 & 1 \\
\hline 2 & 1 & 2 \\
\hline 2 & 3 & 2 \\
\hline 2 & 3 & 2 \\
\hline 2 & 3 & 2 \\
\hline 2 & 3 & 1 \\
\hline 2 & 3 & 2 \\
\hline 4 & 4 & 2 \\
\hline 3 & 3 & 2 \\
\hline 1 & 1 & 1 \\
\hline 2 & 1 & 1 \\
\hline 2 & 3 & 2 \\
\hline 1 & 1 & 1 \\
\hline 1 & 2 & 2 \\
\hline 1 & 1 & 1 \\
\hline 2 & 3 & 4 \\
\hline 2 & 1 & 2 \\
\hline 2 & 4 & 1 \\
\hline 2 & 1 & 1 \\
\hline 2 & 3 & 4 \\
\hline 2 & 1 & 1 \\
\hline 2 & 3 & 1 \\
\hline 2 & 3 & 1 \\
\hline 2 & 2 & 1 \\
\hline 2 & 3 & 1 \\
\hline 2 & 2 & 2 \\
\hline
\end{tabular}

\begin{tabular}{|c|c|c|}
\hline Prioritisation & Level of importance in DSP & Level of importance in DAP \\
\hline 1=Key priority & 1=Performance measure & 1=Performance measure \\
\hline 2=Significant priority & 2=Planned initiative (defined) & 2=Planned initiative (defined) \\
\hline 3=Less important prior & 3=Planned (not defined) & 3=Planned (not defined) \\
\hline 4=Not mentioned & 4=Not mentioned & 4=Not mentioned \\
\hline 4 & 3 & 3 \\
\hline 4 & 3 & 1 \\
\hline 4 & 3 & 3 \\
\hline 4 & 3 & 1 \\
\hline 4 & 3 & 3 \\
\hline 4 & 3 & 3 \\
\hline 4 & 3 & 1 \\
\hline 4 & 4 & 4 \\
\hline 4 & 3 & 1 \\
\hline 4 & 4 & 4 \\
\hline
\end{tabular}


Whanganui DHB

HNA Key recommendations

Smoking

Nutrition/Obesity/Inactivity

Alcohol \& drug issues

Mental health

Cardiovascular disease

Child health

Oral health

Diabetes

Cancer

Respiratory disease

Youth health

Injury prevention

Substandard water supplies

\section{Waitemata DHB}

HNA Key recommendations

Air quality

Waste water

Food borne infectious diseases

Smoking

Nutrition

Obesity

Exercise

Suicide

Alcohol \& drugs

Cancer

Cardiovascular

Diabetes

Oral health

Violence

Mental health

Immunisation

Maori health

Pacific health

Lower socio-economic status

Health information

Primary care

Motor vehicle injuries

Primary/secondary integration

Child health

\begin{tabular}{|c|c|c|}
\hline Prioritisation & Level of importance in DSP & Level of importance in DAP \\
\hline 1=Key priority & 1=Performance measure & 1=Performance measure \\
\hline 2=Significant priority & 2=Planned initiative (defined) & 2=Planned initiative (defined) \\
\hline 3=Less important priority & 3=Planned (not defined) & 3=Planned (not defined) \\
\hline 4=Not mentioned & 4=Not mentioned & 4=Not mentioned \\
\hline 1 & 1 & 2 \\
\hline 1 & 2 & 1 \\
\hline 1 & 2 & 2 \\
\hline 1 & 2 & 1 \\
\hline 1 & 2 & 4 \\
\hline 1 & 2 & 1 \\
\hline 1 & 2 & 1 \\
\hline 1 & 2 & 1 \\
\hline 1 & 2 & 4 \\
\hline 1 & 2 & 3 \\
\hline 1 & 2 & 2 \\
\hline 1 & 2 & 3 \\
\hline 4 & 4 & 4 \\
\hline
\end{tabular}

\begin{tabular}{|c|c|c|}
\hline Prioritisation & Level of importance in DSP & Level of importance in DAP \\
\hline 1=Key priority & 1=Performance measure & 1=Performance measure \\
\hline 2=Significant priority & 2=Planned initiative (defined) & 2=Planned initiative (defined) \\
\hline 3=Less important priority & 3=Planned (not defined) & 3=Planned (not defined) \\
\hline 4=Not mentioned & 4=Not mentioned & 4=Not mentioned \\
\hline 4 & 4 & 4 \\
\hline 4 & 4 & 4 \\
\hline 4 & 4 & 4 \\
\hline 2 & 1 & 3 \\
\hline 2 & 3 & 3 \\
\hline 2 & 3 & 3 \\
\hline 2 & 3 & 3 \\
\hline 2 & 3 & 3 \\
\hline 2 & 3 & 3 \\
\hline 2 & 4 & 4 \\
\hline 1 & 1 & 1 \\
\hline 2 & 1 & 1 \\
\hline 2 & 3 & 1 \\
\hline 4 & 3 & 1 \\
\hline 1 & 2 & 1 \\
\hline 2 & 2 & 1 \\
\hline 1 & 2 & 1 \\
\hline 1 & 3 & 1 \\
\hline 4 & 3 & 2 \\
\hline 1 & 3 & 1 \\
\hline 1 & 3 & 1 \\
\hline 4 & 4 & 4 \\
\hline 1 & 3 & 1 \\
\hline 2 & 3 & 1 \\
\hline
\end{tabular}




\section{Tairawhiti DHB}

HNA Key recommendations

Hth of low socio-economic

Maori health

Smoking

Nutrition/obesity

Suicide

Alcohol \& drug

Cancer

Cardiovascular disease

Diabetes

Oral health

Violence

Child health

Mental health

Unintentional injury

Asthma

Avoidable hospitalisations

Pregnancy complications

Falls $>65 y$ rs

Teenage fertility

Rural health services

Health workforce

Health information

Primary care

Immunisation

Motor vehicle injuries

\section{Counties Manukau DHB}

HNA Key recommendations

Cardiovascular disease

Chronic Obstr. Resp. disease

Diabetes

Infectious disease

Oral health

Child \& Youth health

Elective surgery

Maori health

Mental health (incl A \& D)

Pacific health

Primary care

Public health

\section{Prioritisation}

1=Key priority

Level of importance in DSP Level of importance in DAP

2=Significant priority 2=Planned initiative (defined)

3=Less important priorit 3=Planned (not defined)

4=Not mentioned 4=Not mentioned
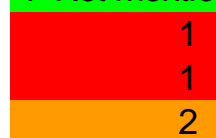

2

2

2

2

2

2

2

2

2

2

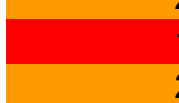

2

2

3

2

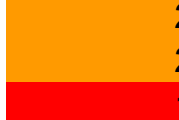

3
3
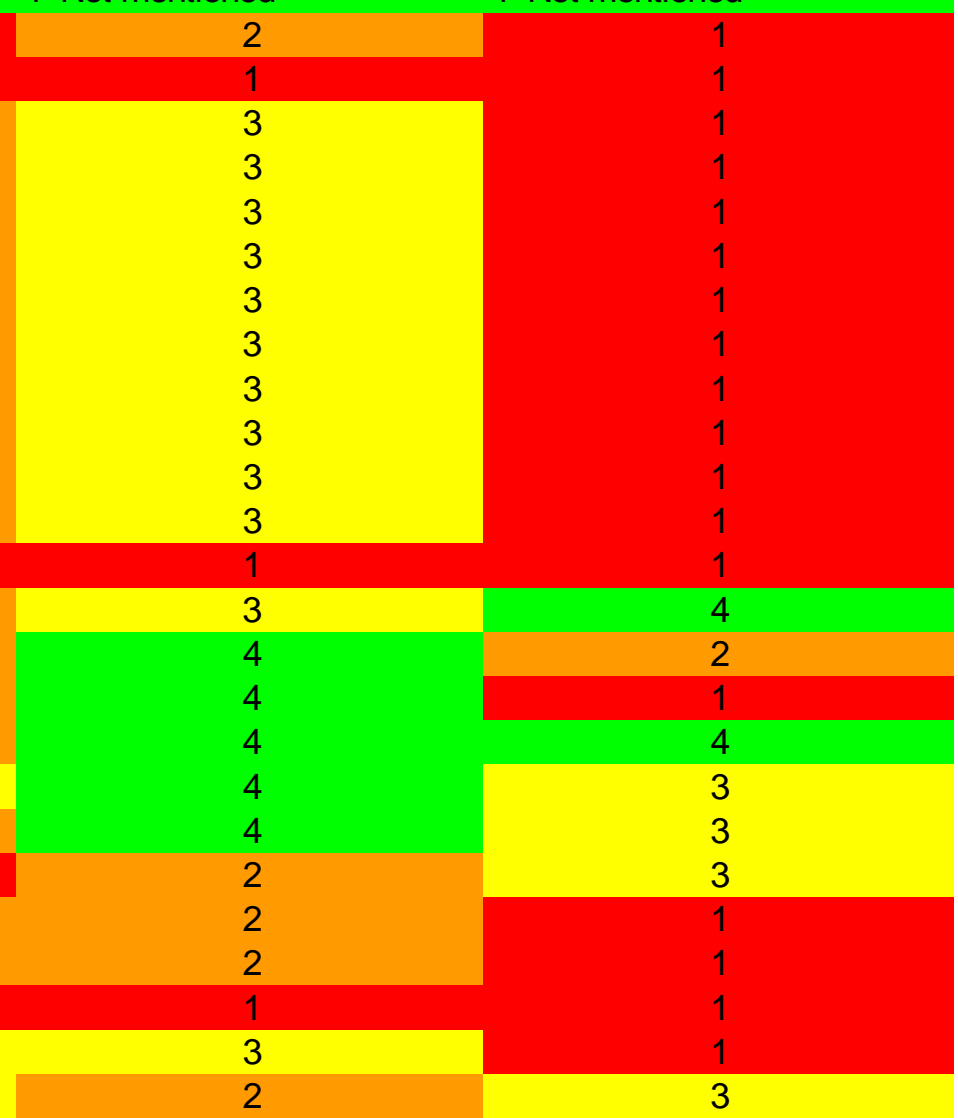

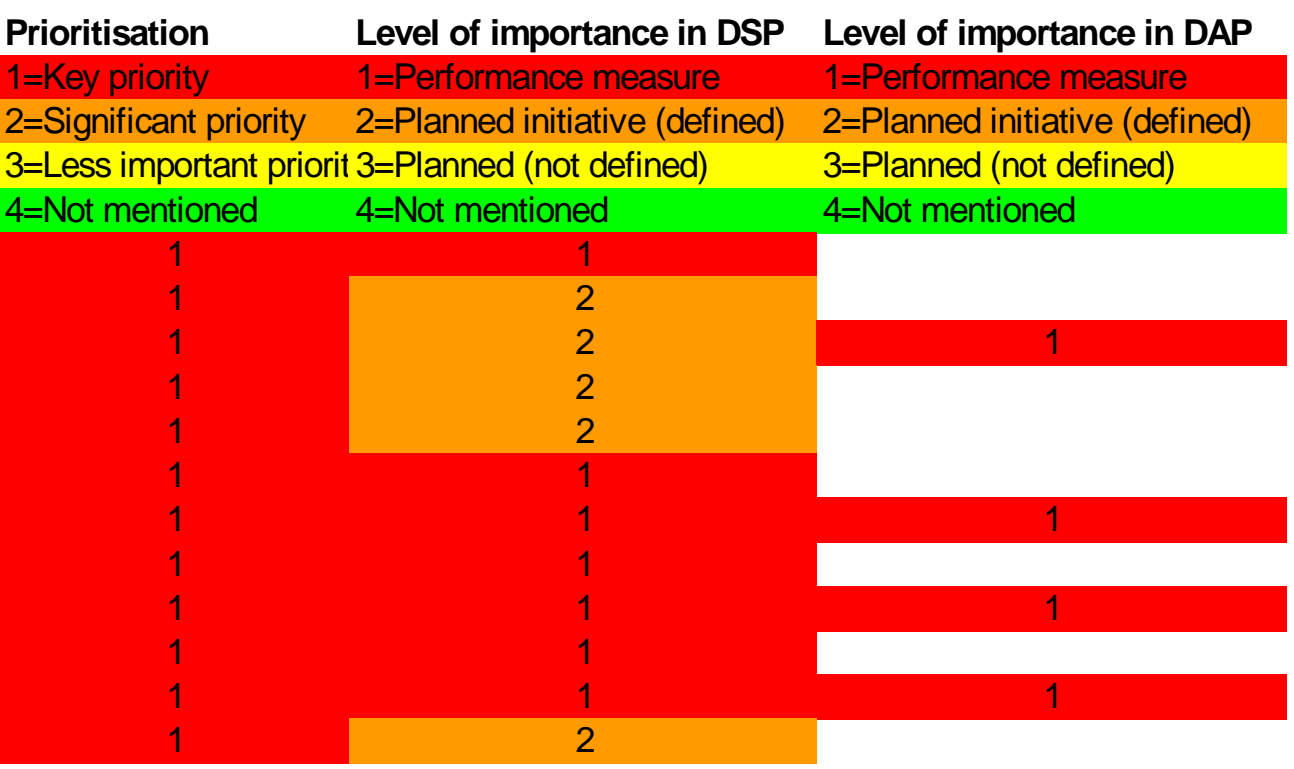




Hutt DHB
HNA Key recommendations
Healthy communities
Maternity
Child \& family
Youth
Cardiovascular
Respiratory
Diabetes
Cancer
Surgical services
Primary care
Mental health
Disability support
Mäori health
Pacific health

Canterbury DHB

HNA Key recommendations Smoking

Nutrition

Obesity

Physical activity

Suicide

Alcohol \& drugs

Cancer

Cardiovascular disease

Diabetes

Oral health

Violence

Child \& youth health

Mäori health

Pacific health

Electives

Emergency services

Rural health

Older persons

Teenage pregnancy

Palliative care

Air quality

Primary health

Mental health

Population health

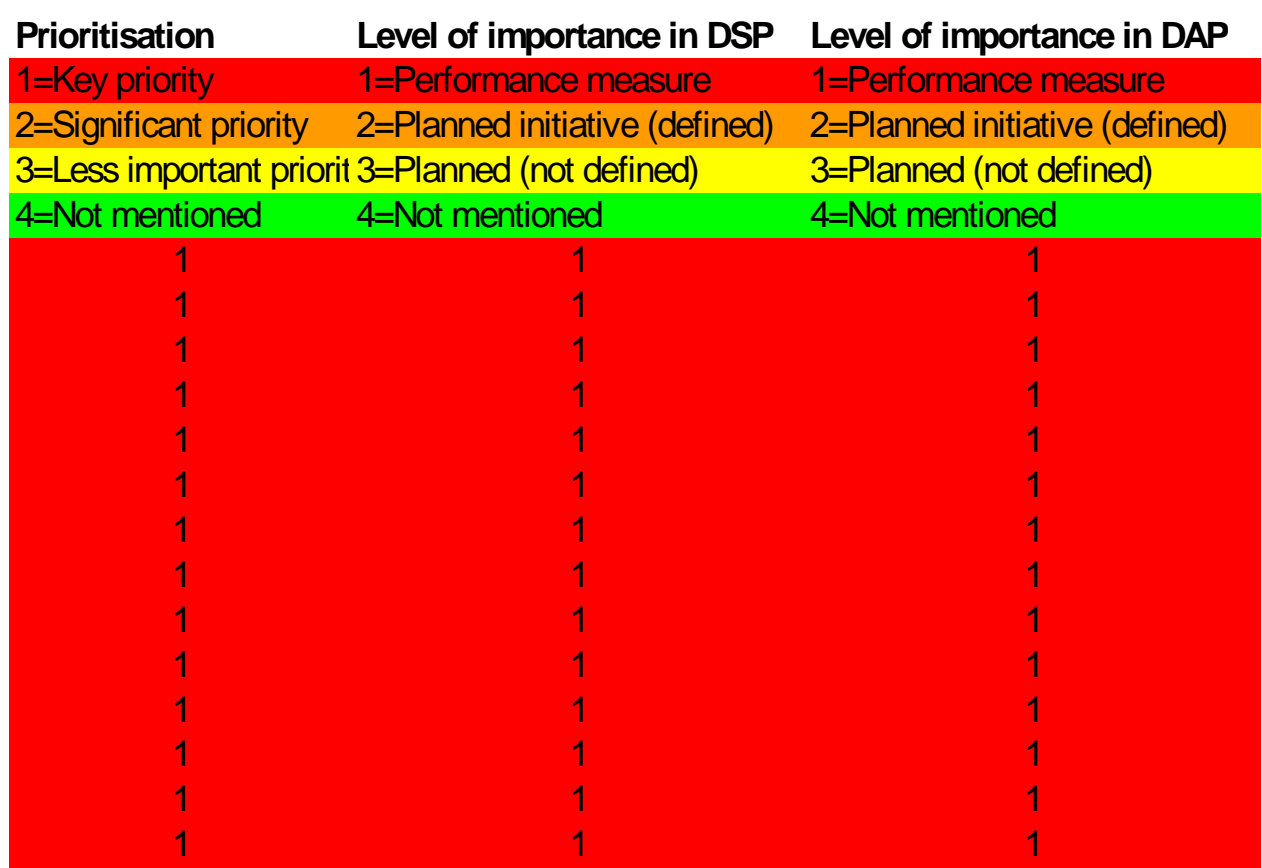

$\begin{array}{lll}\text { Prioritisation } & \text { Level of importance in DSP } & \text { Level of importance in DAP } \\ \text { 1=Key priority } & \text { 1=Performance measure } & \text { 1=Performance measure } \\ \text { 2=Significant priority } & \text { 2=Planned initiative (defined) } & \text { 2=Planned initiative (defined) } \\ \text { 3=Less important priorit 3=Planned (not defined) } & \text { 3=Planned (not defined) } \\ \text { 4=Not mentioned } & \text { 4=Not mentioned } & \text { 4=Not mentioned }\end{array}$

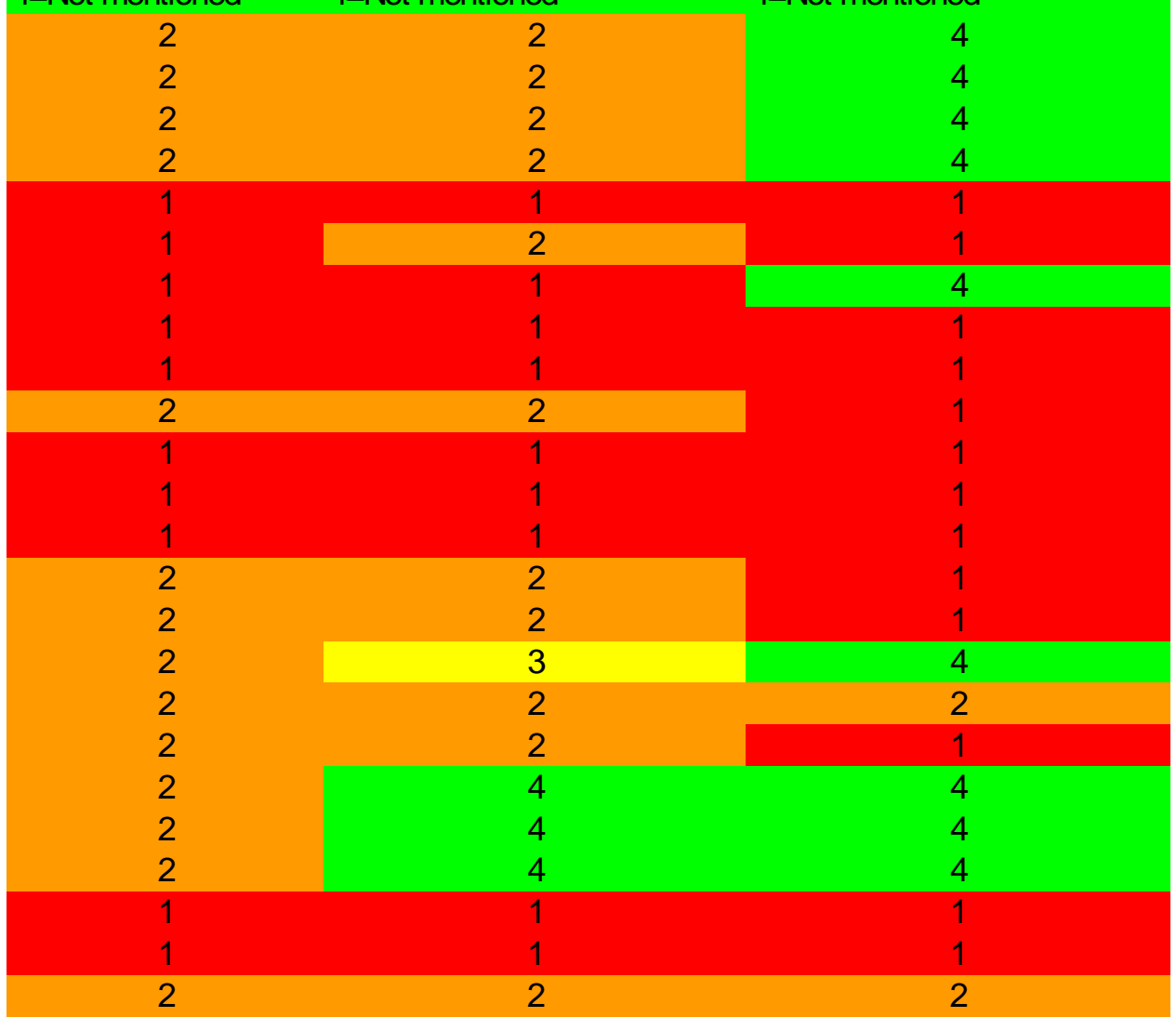




\section{Mid-Central DHB}

HNA Key recommendations

Smoking

Nutrition

Obesity

Physical activity

Suicide

Alcohol \& drugs

Cancer

Cardiovascular disease

Diabetes

Oral health

Violence

Child health

Mental health

Mäori health

Rural health

Environmental health

Avoidable hospitalisations

Primary care

Disability support

Integration

\section{Southland DHB}

HNA Key recommendations

Rural health

Water supplies

Mäori popln health gain

Low socio-economic

Smoking

Alcohol \& drug

Intersectoral action

Primary health

Public health

Data collection

Suicide

Cancer

Cardiovascular disease

Diabetes

Oral health

Child health

Mental health

Service integration

Rationalisation

Pacific popln health gain

Wakatipu district services

Nutrition

Obesity

Physical activity

Violence

\begin{tabular}{|c|c|c|}
\hline Prioritisation & Level of importance in DSP & Level of importance in DAP \\
\hline 1=Key priority & 1=Performance measure & 1=Performance measure \\
\hline 2=Significant priority & 2=Planned initiative (defined) & 2=Planned initiative (defined) \\
\hline \multicolumn{2}{|c|}{ 3=Less important priorit 3=Planned (not defined) } & 3=Planned (not defined) \\
\hline 4=Not mentioned & 4=Not mentioned & 4=Not mentioned \\
\hline 2 & 3 & 3 \\
\hline 2 & 3 & 3 \\
\hline 2 & 3 & 3 \\
\hline 2 & 3 & 3 \\
\hline 2 & 3 & 4 \\
\hline 2 & 3 & 4 \\
\hline 2 & 3 & 1 \\
\hline 2 & 3 & 1 \\
\hline 2 & 3 & 1 \\
\hline 2 & 4 & 4 \\
\hline 3 & 4 & 4 \\
\hline 2 & 4 & 2 \\
\hline 2 & 3 & 1 \\
\hline 2 & 3 & 1 \\
\hline 2 & 3 & 1 \\
\hline 3 & 4 & 4 \\
\hline 4 & 3 & 1 \\
\hline 1 & 3 & 1 \\
\hline 1 & 3 & 1 \\
\hline 1 & 3 & 2 \\
\hline
\end{tabular}

\begin{tabular}{|c|c|c|}
\hline Prioritisation & Level of importance in DSP & Level of importance in DAP \\
\hline 1=Key priority & 1=Performance measure & 1=Performance measure \\
\hline 2=Significant priority & 2=Planned initiative (defined) & 2=Planned initiative (defined) \\
\hline 3=Less important prio & 3=Planned (not defined) & 3=Planned (not defined) \\
\hline 4=Not mentioned & 4=Not mentioned & 4=Not mentioned \\
\hline 1 & 1 & 1 \\
\hline 4 & 4 & 4 \\
\hline 1 & 1 & 1 \\
\hline 4 & 4 & 4 \\
\hline 2 & 2 & 4 \\
\hline 2 & 2 & 2 \\
\hline 4 & 4 & 4 \\
\hline 4 & 4 & 1 \\
\hline 1 & 1 & 2 \\
\hline 4 & 3 & 4 \\
\hline 2 & 2 & 1 \\
\hline 2 & 2 & 4 \\
\hline 2 & 2 & 1 \\
\hline 2 & 2 & 1 \\
\hline 2 & 2 & 1 \\
\hline 2 & 2 & 1 \\
\hline 1 & 2 & 1 \\
\hline 1 & 1 & 1 \\
\hline 1 & 1 & 1 \\
\hline 1 & 1 & 1 \\
\hline 1 & 1 & 1 \\
\hline 2 & 2 & 4 \\
\hline 2 & 2 & 4 \\
\hline 3 & 2 & 4 \\
\hline 2 & 2 & 4 \\
\hline
\end{tabular}




\begin{tabular}{|c|c|c|c|}
\hline \multirow[t]{4}{*}{ Auckland DHB } & Prioritisation & Level of importance in DSP & Level of importance in DAP \\
\hline & 1=Key priority & 1=Performance measure & 1=Performance measure \\
\hline & 2=Significant priority & 2=Planned initiative (defined) & 2=Planned initiative (defined) \\
\hline & 3=Less important priorit & 3=Planned (not defined) & 3=Planned (not defined) \\
\hline HNA Key recommendations & 4=Not mentioned & 4=Not mentioned & 4=Not mentioned \\
\hline Cardiovascular disease & 1 & 2 & \\
\hline Cancer & 1 & 2 & \\
\hline Diabetes & 1 & 3 & \\
\hline Smoking & 1 & 2 & \\
\hline Nutrition & 2 & 2 & \\
\hline Physical exercise & 1 & 2 & \\
\hline Mental health & 1 & 2 & \\
\hline Alcohol \& drug & 1 & 2 & \\
\hline Youth suicide & 1 & 2 & \\
\hline Family violence & 1 & 2 & \\
\hline Infectious diseases & 1 & 2 & \\
\hline Immunisation rates & 1 & 2 & \\
\hline Resp. dis. Admission rates & 1 & 2 & \\
\hline Integration & 4 & 3 & \\
\hline Oral health & 1 & 2 & \\
\hline Mäori health & 4 & 2 & \\
\hline Pacific health & 4 & 2 & \\
\hline Refugee health & 4 & 2 & \\
\hline Primary care & 4 & 2 & \\
\hline Public health & 4 & 2 & \\
\hline Disability services & 4 & 3 & \\
\hline Electives & 1 & 2 & \\
\hline \multirow[t]{4}{*}{ Northland DHB } & Prioritisation & Level of importance in DSP & Level of importance in DAP \\
\hline & 1=Key priority & 1=Performance measure & 1=Performance measure \\
\hline & 2=Significant priority & 2=Planned initiative (defined) & 2=Planned initiative (defined) \\
\hline & 3=Less important priorit & 3=Planned (not defined) & 3=Planned (not defined) \\
\hline HNA Key recommendations & 4=Not mentioned & 4=Not mentioned & 4=Not mentioned \\
\hline Low socio-economic & 1 & 3 & 1 \\
\hline Mäori health & 1 & 2 & 1 \\
\hline Smoking & 2 & 3 & 3 \\
\hline Nutrition & 2 & 3 & 4 \\
\hline Obesity & 2 & 3 & 4 \\
\hline Suicide & 2 & 2 & 1 \\
\hline Alcohol \& drugs & 2 & 2 & 1 \\
\hline Cancer & 2 & 4 & 1 \\
\hline Cardiovascular disease & 2 & 4 & 1 \\
\hline Diabetes & 2 & 4 & 1 \\
\hline Oral health & 1 & 2 & 1 \\
\hline Violence & 2 & 4 & 3 \\
\hline Mental health & 1 & 2 & 1 \\
\hline Avoidable hospitalisation & 2 & 3 & 1 \\
\hline Injuries & 2 & 4 & 3 \\
\hline Cervical screening & 2 & 4 & 4 \\
\hline Water quality & 2 & 4 & 4 \\
\hline Intersectoral action & 1 & 3 & 1 \\
\hline Primary care & 1 & 3 & 1 \\
\hline Rural health & 1 & 2 & 2 \\
\hline Disease management & 1 & 3 & 1 \\
\hline Public health & 1 & 3 & 3 \\
\hline Child \& youth health & 1 & 2 & 1 \\
\hline Disability services & 3 & 2 & 1 \\
\hline
\end{tabular}


Otago DHB

HNA Key recommendations Smoking

Nutrition

Obesity

Physical activity

Suicide

Alcohol \& drugs

Cancer

Cardiovascular disease

Diabetes

Oral health

Reducing violence

Mental illness

Child health

Mäori health

Pacific health

Disability services

Integration

Primary care

Intersectoral

\section{Capital \& Coast DHB}

HNA Key recommendations

Cardiovascular disease

Diabetes

Child health

Suicide

Smoking

Oral health

Mental health

Disability

Maori health

Pacific health

\begin{tabular}{|c|c|c|}
\hline Prioritisation & Level of importance in DSP & Level of importance in DAP \\
\hline 1=Key priority & 1=Performance measure & 1=Performance measure \\
\hline 2=Significant priority & 2=Planned initiative (defined) & 2=Planned initiative (defined) \\
\hline 3=Less important pric & 3=Planned (not defined) & 3=Planned (not defined) \\
\hline 4=Not mentioned & 4=Not mentioned & 4=Not mentioned \\
\hline 1 & 2 & 4 \\
\hline 1 & 2 & 4 \\
\hline 1 & 2 & 4 \\
\hline 1 & 2 & 4 \\
\hline 3 & 2 & 3 \\
\hline 3 & 3 & 3 \\
\hline 3 & 4 & 1 \\
\hline 3 & 4 & 1 \\
\hline 3 & 1 & 1 \\
\hline 3 & 2 & 1 \\
\hline 3 & 3 & 3 \\
\hline 2 & 2 & 1 \\
\hline 1 & 2 & 1 \\
\hline 1 & 1 & 1 \\
\hline 2 & 1 & 2 \\
\hline 1 & 2 & 1 \\
\hline 1 & 2 & 1 \\
\hline 1 & 3 & 1 \\
\hline 2 & 3 & 1 \\
\hline
\end{tabular}

\begin{tabular}{|c|c|c|}
\hline Prioritisation & Level of importance in DSP & Level of importance in DAP \\
\hline 1=Key priority & 1=Performance measure & 1=Performance measure \\
\hline 2=Significant priority & 2=Planned initiative (defined) & 2=Planned initiative (defined) \\
\hline $3=$ Less important prior & 3=Planned (not defined) & 3=Planned (not defined) \\
\hline 4=Not mentioned & 4=Not mentioned & 4=Not mentioned \\
\hline 1 & 3 & 1 \\
\hline 1 & 3 & 1 \\
\hline 1 & 3 & 1 \\
\hline 1 & 3 & 4 \\
\hline 1 & 3 & 4 \\
\hline 1 & 3 & 1 \\
\hline 1 & 3 & 1 \\
\hline 1 & 3 & 1 \\
\hline 2 & 3 & 1 \\
\hline 2 & 3 & 1 \\
\hline
\end{tabular}


Appendix Thirteen

\begin{tabular}{|c|c|c|c|}
\hline \multirow[t]{4}{*}{ Hawke's Bay DHB } & Prioritisation & Level of importance in DSP & Level of importance in DAP \\
\hline & 1=Key priority & 1=Performance measure & 1=Performance measure \\
\hline & 2=Significant priority & 2=Planned initiative (defined) & 2=Planned initiative (defined) \\
\hline & 3=Less important priori & t 3=Planned (not defined) & 3=Planned (not defined) \\
\hline HNA Key recommendations & 4=Not mentioned & 4=Not mentioned & 4=Not mentioned \\
\hline Maori health & 2 & 1 & 1 \\
\hline Intersectoral action & 2 & 3 & 2 \\
\hline Smoking & 1 & 1 & 2 \\
\hline Hlth status low socio-economic & 1 & 1 & 2 \\
\hline Health edn \& promotion & 2 & 3 & 1 \\
\hline Primary care & 2 & 2 & 1 \\
\hline Rural health & 2 & 3 & 2 \\
\hline Primary/secondary interface & 2 & 3 & 2 \\
\hline Health information & 2 & 2 & 1 \\
\hline Nutrition/obesity & 2 & 3 & 4 \\
\hline Suicide & 2 & 2 & 1 \\
\hline Alcohol \& drug & 2 & 3 & 2 \\
\hline Cancer & 2 & 1 & 2 \\
\hline Cardiovascular & 1 & 1 & 1 \\
\hline Diabetes & 2 & 1 & 1 \\
\hline Oral health & 1 & 1 & 1 \\
\hline Violence & 1 & 2 & 3 \\
\hline Child health & 1 & 1 & 1 \\
\hline Mental health & 2 & 1 & 1 \\
\hline Non-intentional injury & 1 & 1 & 3 \\
\hline Asthma & 1 & 1 & 1 \\
\hline Hearing & 1 & 1 & 1 \\
\hline Immunisation & 1 & 1 & 1 \\
\hline Post natal mortality & 2 & 4 & 4 \\
\hline Teenage pregnancies & 2 & 1 & 2 \\
\hline Sexually transmitted disease & 2 & 1 & 2 \\
\hline Workforce development & 2 & 2 & 1 \\
\hline Avoidable hospitalisations & 2 & 1 & 1 \\
\hline Confidence in health services & 1 & 2 & 1 \\
\hline
\end{tabular}

\section{West Coast DHB}

HNA Key recommendations Hlth of low socio-economic Maori health

Avoidable hospitalisations Intersectoral action

Smoking

Alcohol \& drug

Primary care

Rural health

\begin{tabular}{|c|c|c|}
\hline Prioritisation & Level of importance in DSP & Level of importance in DAP \\
\hline 1=Key priority & 1=Performance measure & 1=Performance measure \\
\hline 2=Significant priority & 2=Planned initiative (defined) & 2=Planned initiative (defined) \\
\hline 3=Less important priori & 3=Planned (not defined) & 3=Planned (not defined) \\
\hline 4=Not mentioned & 4=Not mentioned & 4=Not mentioned \\
\hline 4 & 3 & 3 \\
\hline 1 & 1 & 1 \\
\hline 4 & 3 & 4 \\
\hline 4 & 2 & 2 \\
\hline 1 & 2 & 2 \\
\hline 2 & 3 & 1 \\
\hline 1 & 1 & 1 \\
\hline 4 & 3 & 3 \\
\hline
\end{tabular}




\section{Appendix 14: Review criteria template for evaluation of the Quality of DHB HNAs}

\begin{tabular}{|c|c|c|c|c|}
\hline 1 & Is consistent with the approach in HNA for New Zealand: An Overview and Guide, Dec. 2000 & $\begin{array}{l}\text { Not } \\
\text { met }\end{array}$ & $\begin{array}{l}\text { Partly } \\
\text { met }\end{array}$ & $\begin{array}{l}\text { Fully } \\
\text { met }\end{array}$ \\
\hline 1.1 & The objectives of the HNA exercise are clearly laid out & 0 & 1 & 2 \\
\hline 1.2 & Data are presented on the base socio-demographic and geographical characteristics of the population & 0 & 1 & 2 \\
\hline 1.3 & Wide range of data regarding expressed demand for health care services is presented & 0 & 1 & 2 \\
\hline 1.4 & Data analysis identifies gaps where there are needs for services & 0 & 1 & 2 \\
\hline 1.5 & Forecasting needs for future services is undertaken & 0 & 1 & 2 \\
\hline 2 & Gives particular attention to NZHS population health priorities & & & \\
\hline 2.1 & Epidemiological data related to each of the 13 priority population health objectives are provided & 0 & 1 & 2 \\
\hline 2.2 & Data are reported for Māori and Pacific peoples separately & 0 & 1 & 2 \\
\hline 3 & Identifies those groups expressing poorer health outcomes & & & \\
\hline 3.1 & Identifies Māori experiencing poorer health outcomes & 0 & 1 & 2 \\
\hline 3.2 & Identifies Pacific people experiencing poorer health outcomes & 0 & 1 & 2 \\
\hline 3.3 & Identifies low socio-economic people experiencing poorer health outcomes & 0 & 1 & 2 \\
\hline 3.4 & Identifies other groups experiencing poorer health outcomes & 0 & 1 & 2 \\
\hline 4 & Lists providers, services and number of patients receiving such services giving particular attention to NZHS priorities & & & \\
\hline 4.1 & A stocktake of the numbers, availability and distribution of health care providers is presented & 0 & 1 & 2 \\
\hline 4.2 & Information regarding the capacity of services provided by health care providers is presented & 0 & 1 & 2 \\
\hline 4.3 & Intersectoral data regarding service providers related to health are presented & 0 & 1 & 2 \\
\hline 5 & Considers public health measures and actions on the wider determinants of health & & & \\
\hline 5.1 & Environmental factors causing morbidity are identified & 0 & 1 & 2 \\
\hline 5.2 & Need for public health measures and actions are identified & 0 & 1 & 2 \\
\hline 6 & Involves participation and targeted consultation with community groups and Māori (\& Pacific people where appropriate) & & & \\
\hline
\end{tabular}




\begin{tabular}{|c|c|c|c|c|}
\hline 6.1 & Participation by Māori in the process is clearly identified & 0 & 1 & 2 \\
\hline 6.2 & Targeted consultation with Māori and Pacific peoples has taken place & 0 & 1 & 2 \\
\hline 6.3 & Appropriate targeted consultation with community groups has taken place & 0 & 1 & 2 \\
\hline \multicolumn{5}{|c|}{ Part B Evaluation of other features of the HNAs } \\
\hline & & No & Partly & Yes \\
\hline 7 & Discussion of the meaning of need & 0 & 1 & 2 \\
\hline 8 & Was there stakeholder involvement? & 0 & 1 & 2 \\
\hline 9 & Was the methodology stated (including sources of data)? & 0 & 1 & 2 \\
\hline 10 & Presence of all significant data? (includes primary care, mental health etc) & 0 & 1 & 2 \\
\hline 11 & Is there a set of recommendations arising from the HNA? & 0 & 1 & 2 \\
\hline 12 & Participation in the Wellington Clinical School regional HNA process? & No & & Yes \\
\hline
\end{tabular}




\section{References}

Abramson, J.H. (1988) 'Community-orientated primary care - Strategy, approaches, and practice: A review', Public Health Reviews, 16: 35-98.

Ajwani, S., Blakely, T., Robson, B., Tobias, M. and Bonne, M. (2003) Decades of Disparity: Ethnic mortality trends in New Zealand 1980-1999. Ministry of Health and University of Otago, Wellington.

Annett, H. and Rifkin, S. (1988) Improving Urban Health. Geneva: World Health Organisation.

Anonymous (2002) Community needs assessment of lower Manhattan residents following the World Trade Center attacks - Manhattan, New York City, 2001. Atlanta: Center for Disease Control.

Ashton, T. (1996) Contracting for health services in New Zealand: early experiences. In Proceedings of International Health Economics Association Inaugural Conference 1996, Vancouver.

Ashton, T. (1999) 'The Health Reforms: To Market and Back?' in J. D. Boston, P. Dalziel and S. S. John (eds), Redesigning the Welfare State in New Zealand Problems, Policies, Prospects. Auckland: Oxford University Press.

Ashton, T., Cumming, J. and Devlin, N. (1999) Prioritising health and disability support services: principles, processes and problems. Wellington: National Health Committee.

Ashton, T., Cumming, J. and Devlin, N. (2000) 'Priority-setting in New Zealand: translating principles into practice', Journal of Health Services Research and Policy, 5: $170-5$.

Audit Commission (1993) Their health, your business: the new role of the District Health Authority. HMSO, London.

Barbour, R.S. (1999) 'The case for combining qualitative and quantitative approaches in health services research', Journal of Health Services Research \& Policy, 4: 39-43.

Batterham, R. and Jordan, H. (1997) Current accepted practice in the assessment of community health needs. Melbourne: Support and Evaluation Resource Unit: Centre for Health Program Evaluation, University of Melbourne.

Bay of Plenty Area Health Board (1992) 1992 Health Status Review. Tauranga: Bay of Plenty Area Health Board.

Beech, R., Guilliford, M., Mays, N., Melia, J. and Roderick, P. (1997) 'Renal Disease', in A. Stevens and J. Raftery (eds), Health care needs assessment: the epidemiologically based reviews. Vol. 1. Oxford: Radcliffe Press. 
Benveniste, G. (1989) Mastering the Politics of Planning: Crafting Credible Plans and Policies that Make a Difference. San Francisco: Jossey-Bass.

Bohmer, P. (1996) Programme budgeting and marginal analysis for the allocation of resources within services for respiratory disease. Christchurch: Southern Regional Health Authority.

Bowling, A. (1997) Research Methods in Health: Investigating Health and Health Services. Buckingham: Open University Press.

Bradshaw, J. (1972a) 'The concept of social need', New Society, March: 640-3.

Bradshaw, J. (1972b) 'A taxonomy of social need', in G. McLaghlan (eds), Problems and Progress in Medical Care: Essays on Current Research. London: Oxford University Press.

Brambleby, P. (1995) 'A survivor's guide to programme budgeting', Health Policy, 33: 127-45.

Brander, P. (1991) Adolescent sexual practices: a study of sexual experiences and service needs among a group of New Zealand adolescents. Wellington: Department of Health.

Brander, P. and Norton, V. (1993) Women living with HIV/AIDS: issues and needs confronting women with HIV/AIDS. Wellington: Ministry of Health.

Brewer, J. and Hunter, A. (1989) Multimethod Research: A Synthesis of Styles. Newbury Park, CA: Sage.

Bromley Health (1995) Healthy Crays Project. Bromley: Bromley Health Authority.

Buetow, S. and Coster, G. (2001) 'Looking forward to health needs assessments: a new perspective on 'need", New Zealand Medical Journal, 114: 92-4.

Buetow, S. and Kerse, N. (2001) 'Does reported health promotion activity neglect people with ill-health?' Health Promotion International, 16: 73-8.

Burgess, R.G. (1984) In the field: An introduction to field research. London: Routledge.

Campbell, S., Roland, M. and Buetow, S. (2000) 'Defining quality of care', Social Science and Medicine, 51: 1611-25.

Carpinter, A. (1989) Needs assessment demystified. A practical guide to identifying need and deciding what to do about it. Draft. Wellington: Rainbow Research.

Cashman, S.B., Fulmer, H.S. and Staples, L. (1994) 'Community health: beyond care for individuals', Social Policy, 24: 52-62.

Central Regional Health Authority (1994) Strong links: building better services to meet the health and disability support service needs of people in Porirua. Wellington:

Central Regional Health Authority. 
Central Regional Health Authority (1996) Poutama whirinaki=Interwoven paths: the report of the Central RHA Wanganui Needs Assessment. Wellington: Central Regional Health Authority.

Chalmers, I., Dickerson, K. and Chalmers, T.C. (1992) 'Getting to grips with Archie Cochrane's agenda', British Medical Journal, 305: 786.

Chambers, R. (1981) 'Rapid rural appraisal: Rationale and repertoire', Public Administration \& Development, 1: 95-100.

Cheung, J. and Health \& Disability Analysis Unit (1996) Report on resource equity for the people of the Lakes Sub-Region. Hamilton: Midland Health.

Clarke, D., Coster, G., Reid, P. and Scott, J. (2002) Far North Services Review (by The Independent Review Team). Report to the Ministry of Health, Northland DHB and the Far North Community, Auckland.

Cohen, D. (1994) 'Marginal analysis in practice: an alternative to needs assessment for contracting health care', British Medical Journal, 309: 781-4.

Congdon, P. (2001) 'Health status and healthy life measures for population health need assessment: modelling variability and uncertainty', Health and Place, 7: 13-25.

Controller and Auditor-General (2002) Report of the Controller and Auditor-General on Purchasing Primary Health Care Provided in General Practice. Wellington: Office of the Contoller and Auditor-General.

Coopers \& Lybrand (1995) Prophylactic treatment for severe haemophilia A: an assessment of the costs and benefits. Auckland: Ministry of Health.

Coster, G. (2000) Health Needs Assessment for New Zealand: Background paper and literature review. Wellington: Ministry of Health.

Coster, G. and Buetow, S. (2002) 'Challenges for District Health Boards as needs assessors', New Zealand Medical Journal, 115: 298-300.

Coster, G. and Gribben, B. (1999) Primary care models for delivering population-based health outcomes. Wellington: National Advisory Committee on Health and Disability.

Coster, H.M. (1999) Community Responses to Changes in Delivery of Rural Health Services in Balclutha and Dannevirke. MA Thesis, University of Auckland. Auckland.

Crampton, P. (1999) Third sector primary health care. Wellington: National Advisory Committee on Health and Disability.

Crampton, P. and Laugesen, M. (1995) The use of indices of need in resource allocation formulae for primary health care. Wellington: Health Services Research Centre, Victoria University of Wellington. 
Crawley, L., Pulotu-Endemann, F.K. and Stanley-Findlay, R.T.U. (1995) Strategic directions for the mental health services for Pacific Island people. Wellington: Ministry of Health.

Crown Company Monitoring Advisory Unit Health Group (2000) External Accountability and Reporting by DHBs in a Unitary Structure. Wellington: Crown Company Monitoring Advisory Unit.

Cumming, J., Ashton, T., Barnett, P., Scott, P., Kiro, C., Cunningham, C., Bolton, A., Russell, M., Tenbensel, T., Powell, M. and Coster, G. (2001) Assessing governance, purchasing and accountability in the NZ health care sector. Wellington: HRC Grant. Health Services Research Centre, Victoria University of Wellington.

Cumming, J., Hawkins, L. and Jensen, K. (1996) Resource allocation in the reformed New Zealand health sector. In Proceedings of the Economics and Health Conference 1995, School of Health Services Management, University of New South Wales Sydney.

Cumming, J. and Salmond, G. (Eds.) (1998) Reforming New Zealand health care. New York: Addison Wesley Longman.

Davidoff, F., Haynes, B., Sackett, D. and Smith, R. (1995) 'Evidence based medicine: a new health services research journal to help doctors to identify the information they need', British Medical Journal, 310: 1085-6.

Davis, P., Street, A. and Posnett, J. (1995) Programme budgeting: a disease based approach to describing health care activity and expenditure. Consortium report $P 2002 b$. York: York Health Economics Consortium, University of York.

Delamont, S. (1992) Fieldwork in educational settings. Methods, pitfalls and perspectives. London: The Falmer Press.

Deloitte Ross Tohmatsu (1991) An investigation into the special health care needs of refugees for the Auckland Area Health Board. New Zealand: Deloitte Ross Tohmatsu.

Denzin, N.K. (1970) The research act: A theoretical introduction to sociological methods. Chicago: Aldine Publishing Co.

Department of Health (1993a) Assessment of geographical access to primary care providers: Area Health Board report: data tables on Auckland Area Health Board. Wellington: Department of Health.

Department of Health (1993b) Assessment of geographical access to primary care providers: Area Health Board report: data tables on Bay of Plenty Area Health Board. Wellington: Department of Health.

Department of Health (1993c) Assessment of geographical access to primary care providers: Area Health Board Report: data tables on Hawkes Bay Area Health Board. Wellington: Department of Health. 
Department of Health (1993d) Assessment of geographical access to primary care providers: Area Health Board Report: data tables on Manawatu-Wanganui Area Health Board. Wellington: Department of Health.

Department of Health (1993e) Assessment of geographical access to primary care providers: Area Health Board Report: data tables on Nelson-Marlborough Area Health Board. Wellington: Department of Health.

Department of Health (1993f) Assessment of geographical access to primary care providers: Area Health Board report: data tables on Northland Area Health Board. Wellington: Department of Health.

Department of Health (1993g) Assessment of geographical access to primary care providers: Area Health Board Report: data tables on West Coast Area Health Board. Wellington: Department of Health.

Department of Health (1993h) Assessment of geographical access to primary care providers: Area Health Board Report: data tables on Canterbury Area Health Board. Wellington: Department of Health.

Department of Health (1993i) Assessment of geographical access to primary care providers: Area Health Board Report: data tables on Otago Area Health Board. Wellington: Department of Health.

Department of Health (1993j) Assessment of geographical access to primary care providers: Area Health Board Report: data tables on Southland Area Health Board. Wellington: Department of Health.

Department of Health (1993k) Assessment of geographical access to primary care providers: Area Health Board Report: data tables on Taranaki Area Health Board. Wellington: Department of Health.

Department of Health (19931) Assessment of geographical access to primary care providers: Area Health Board Report: data tables on Waikato Area Health Board. Wellington: Department of Health.

Department of Health (1993m) Assessment of geographical access to primary care providers: Area Health Board Report: data tables on Wellington Area Health Board. Wellington: Department of Health.

Department of Health (1993n) Public Health Common Data Set. University of Surrey, Surrey.

Dingwall, R., Murphy, E., Watson, P., Greatbatch, D. and Parker, S. (1998) 'Catching goldfish: quality in qualitative research', Journal of Health Services Research \& Policy, 3: 167-72.

Dixon, J. and Welch, H.G. (1991) 'Priority setting: lessons from Oregon', Lancet, 337: 891-4. 
Donabedian, A. (1980) Explorations in quality assessment and monitoring. Vol 1. The definition of quality and approaches to quality assessment. Ann Arbor, Michigan: Health Administration Press.

Donaldson, C. and Mooney, G. (1991) 'Needs assessment, priority setting, and contracts for health care: an economic view', British Medical Journal, 303: 1529-30.

Donaldson, C., Walker, A. and Craig, N. (1995) Programme Budgeting and Marginal Analysis: A Handbook for applying economics in health care purchasing. Glasgow: Scottish Needs Assessment Programme, Scottish Forum for Public Health Medicine.

Donaldson, L.J. (1998) A first class service: quality in the new NHS. Department of Health, London.

Drummond, M. (1995) 'The United Kingdom National Health Service reforms: where are we now?' Australian Health Review, 18: 28-42.

Dunt, D. (1999) Health care needs assessment study: South Island West Coast. Melbourne: Coast Health Care and the Health Funding Authority.

Durie, M. (1994) Whaiora: Maori Health Development. 2nd edition. Auckland: Oxford University Press.

Ealing Health Agency (1995) Locality Commissioning. Ealing:

Eddy, D.M. (1994) 'Clinical decision making: from theory to practice. Principles for making difficult decisions in difficult times', The Journal of the American Medical Association, 271: 1792-8.

Erickson, P., Kendall, E., Anderson, J. and Kaplan, R. (1989) 'Using composite health status measures to assess the nation's health', Medical Care, 27: S66-76.

Etizioni, A. (1967) 'Mixed scanning: a third approach to decision-making', Public Administration Review, 27: 385-92.

Ferguson, B. and Ryder, S. (1991) Future role of the District Health Authority: assessing needs for services and setting priorities. York:: CHE Discussion Paper 87.

Fisher, B. and Gillam, S. (1999) 'Community development in the new NHS', British Journal of General Practice, 49: 428-30.

Fisher, B., Neve, H. and Heritage, Z. (1999) 'Community development, user involvement, and primary health care', British Medical Journal, 318: 749-50.

Fletcher, P.C. and Hirdes, J.P. (2001) 'Assessing the health and functional status of older women with breast cancer using the minimum data set-home care (MDS-HC)', Canadian Journal of Public Health, 92: 457-9.

Fontana, A. and Frey, J.H. (1994) 'Interviewing: the art of science', in N. K. Denzin and Y. S. Lincoln (eds), Handbook of Qualitative Research. Thousand Oaks, CA: Sage. 
Frankel, S. (1991a) 'The epidemiology of indications', Journal of Epidemiology and Community Health, 45: 257-9.

Frankel, S. (1991b) 'Health needs, health-care requirements, and the myth of infinite demand', Lancet, 337: 1588-90.

Frankel, S., Ebrahim, A. and Smith, G.D. (2000) 'The limits to demand for health care', British Medical Journal, 321: 40-5.

Freeman, R., Gillam, S., Shearin, C. and Pratt, J. (1997) Community Development and Involvement in Primary Care. A guide to involving the community in COPC.

London: Kings' Fund.

Fuller, J., Bentley, M. and Shotton, D. (2001) 'Use of community health needs assessment for regional planning in country South Australia', Australian Journal of Rural Health, 9: 12-7.

Fulop, N. and Hensher, M. (1997) A survey of needs assessment activity in London Health Authorities. London: Kings' Fund.

Garr, D., Rhyne, R. and Kukulka, G. (1993) 'Incorporating a community-orientated approach in primary care', American Family Physician, 47: 1699-702.

Giacomini, M.K. and Cook, D.J. (2000) 'Users' guides to the medical literature: XXIII. Qualitative research in health care A. Are the results of the study valid?' The Journal of the American Medical Association, 284: 357-62.

Gibbs, A., Fraser, D. and Scott, J. (1988) Unshackling the Hospitals. Report of the Hospital and Related Services Taskforce. Wellington:

Gillam, S. (1992) 'Assessing the health care needs of populations - the general practitioner's contribution (editorial)', British Journal of General Practice, 42: 404-5.

Gillam, S.J. and Murray, S.A. (1996) Needs assessment in general practice. London: The Royal College of General Practitioners.

Good Health Wanganui (2000a) An assessment of health needs for the Good Health Wanganui Region, Phase 1. Wanganui: Good Health Wanganui and the Wanganui District Council.

Good Health Wanganui (2000b) An Assessment of the Health Needs of the Good Health Wanganui Region. Wanganui.

Government Committee on Choices in Health Care (1992) Choices in health care: a report by the Government Committee on choices in health care. Rijswijk, The Netherlands: Ministry of Health, Welfare and Cultural Affairs.

Ham, C. (1993) 'Priority setting in the NHS: reports from six districts', British Medical Journal, 307: 435-8. 
Hammersley, M. and Atkinson, P. (1983) Ethnography: Principles in practice. London: Routledge.

Hanlon, P., Murie, J., McEwen, J., Moir, D. and Russell, E. (1998) 'A study to determine how health needs assessment is being used to improve health', Public Health, 112: 343-6.

Hanrahan, M.C. (2002) 'Identifying the needs of Innu and Inuit patients in urban health settings in Newfoundland and Labrador', Canadian Journal of Public Health, 93: $149-52$.

Harris, A. (Ed.) (1997) Needs to know: a guide to needs assessment for primary care. London: Churchill Livingstone.

Harris, A. and Marshall, T. (1997) 'The language of needs assessment', in A. Harris (eds), Needs to know: a guide to needs assessment in primary care. London: Churchill Livingstone.

Hawe, P. (1996) 'Needs assessment must become more change-focussed', Australian and New Zealand Journal of Public Health, 20: 473-8.

Health \& Disability Analysis Unit Midland Health (1993) Midland Health Community Health Survey 1993. Midland Regional Health Authority, Hamilton.

Health \& Disability Analysis Unit Midland Health (1995) The people of the Midland Health Region, Volume 2: Health Status, Part 1: Infants and Children. Midland Health, Hamilton.

Health \& Disability Analysis Unit Midland Health (1996) The people of the Midland Health Region, Volume 2: Health Status, Part II: The Health Status of Young People. Midland Health, Hamilton.

Health \& Disability Analysis Unit Midland Health (1997a) Family Health Services in the Midland Region. Midland Health, Hamilton.

Health \& Disability Analysis Unit Midland Health (1997b) Sexual and Reproductive Health in the Midland Health Region. Midland Health, Hamilton.

Health \& Disability Analysis Unit Midland Health (1998) Pregnancy \& Childbirth in the Midland Region. Midland Health Funding Authority, Hamilton.

Health Funding Authority (1998a) The health of the people in the south: West Coast, Canterbury, Otago, Southland. Dunedin: Health Funding Authority.

Health Funding Authority (1998b) How shall we prioritise Health and Disability Services? A discussion paper, internal paper (May). Wellington: Health Funding Authority.

Health Funding Authority (1998c) Prioritisation Methodology and Process. Wellington: Health Funding Authority. 
Health Funding Authority (1998d) Priority setting and resource allocation. Paper prepared for the Health Funding Authority Board. Wellington: Health \& Disability Analysis Unit, Midland Division of the Health Funding Authority.

Health Funding Authority (1999) Kia Tu Kia Puawai. Wellington: Health Funding Authority.

Health Funding Authority (2000a) Aged Residential Care Utilisation in the Mid-North Island. Health Funding Authority, Hamilton.

Health Funding Authority (2000b) Health Funding Authority Consultation Obligations and Guidelines. 1st edn. Wellington: Health Funding Authority.

Health Funding Authority (2000c) Improving our health in Wellington. Wellington: Health Funding Authority.

Health Funding Authority (2000d) Overview of the Health Funding Authority's prioritisation decision making framework. Wellington: Health Funding Authority.

Health Funding Authority (2000e) Procedure for prioritising new service initiatives. Wellington: Health Funding Authority.

Health Funding Authority and Ministry of Health (1999) Disability in New Zealand: Overview of the 1996/97 Surveys. Wellington: Health Funding Authority and Ministry of Health.

HealthSearch (1998) Report to the Ministry of Health: Health Profile of the Wellington Region. Wellington.

Hefford, M. (2002) personal communication.

Hensher, M. and Fulop, N. (1999) 'The influence of health needs assessment on health care decision-making in London health authorities', Journal of Health Service Research \& Policy, 4: 90-5.

Hill, M. (1997) The Policy Process in the Modern State. 3rd Edn. Hertfordshire: Prentice Hall Harvester Wheatsheaf.

Hodder, I. (1994) 'The interpretation of documents and material culture.' in N. K. Denzin and Y. S. Lincoln (eds), Handbook of Qualitative Research. London: Sage.

Honigsbaum, F. (1991) Who shall live? Who shall die? Oregon's health financing proposals. London: King's Fund College Papers.

Hooper, J. and Longworth, P. (1997) Health needs assessment in primary care. A workbook for primary health care teams. Wakefield: Calderdale and Kerklees Health Authority.

Hopton, J.L. and Dlugolecka, M. (1995a) 'Need and demand for primary health care: a comparative survey approach', British Medical Journal, 310: 1369-73. 
Hopton, J.L. and Dlugolecka, M. (1995b) 'Patients perceptions of need for primary care health services: useful for priority setting', British Medical Journal, 310: 1237-40.

Hornblow, A. and Barnett, P. (2000) 'A turbulent decade: lessons from the 'health reforms", NZ Med J, 113: 133-4.

Hulse, P. (1999) Local Community Input into Health. Speech to the Independent Practitioners Association Annual Conference, Waitakere City.

Hutt Valley District Health Board (2002) Towards a Healthier Community: The Five Year District Strategic Plan 1 July 2002 to 30 June 2007. Hutt Valley District Health Board, Hutt Valley.

J Le Grand and Bartlett, W. (Eds.) (1993) Quasi-markets and Social Policy. Basingstoke: Macmillan.

Jackson, G., Palmer, C., Lindsay, A. and Peace, J. (2001) Counties Manukau Health Profile. Manukau City: Counties Manukau District Health Board.

Jewkes, R. and Murcott, A. (1996) 'Meanings of community', Social Science \& Medicine, 43: 555-63.

Jones, S. (1985) 'Depth interviewing', in R. Walker (eds), Applied Qualitative Research. Aldershot: Gower.

Jordan, J., Dowswell, T., Harrison, S., Lilford, R.J. and Mort, M. (1998) 'Health needs assessment. Whose priorities? Listening to users and the public', British Medical Journal, 316: 1668-70.

Jordan, J. and Wright, J. (1997) 'Making sense of health needs assessment', British Journal of General Practice, 48: 695-6.

Jordan, J., Wright, J., Ayers, P., Hawkings, M., Thomson, R., Wilkinson, J. and Williams, R. (2002) 'Health needs assessment and needs-led health service change: a survey of projects involving public health doctors', Journal of Health Services Research \& Policy, 7: 71-80.

Jordan, J., Wright, J., Wilkinson, J. and Williams, D.R.R. (1996) Health needs assessment in primary care: a study of understanding and experience in three districts. Leeds: Nuffield Institute for Health.

Jupp, V. (1996) 'Documents and critical research', in R. Sapsford and V. Jupp (eds), Data Collection and Analysis. London: Sage.

Kark, S.L. and J H Abramson (eds) (1981) 'Community-focussed health care', Israel Journal of Medical Sciences, 17: 65-221.

Kark, S.L. and Kark, E. (1983) 'An alternative strategy in community health care: community-orientated primary health care', Israel Journal of Medical Sciences, 19: 707-13. 
Kearns, R.A. (1997) 'Narrative and metaphor in health geographies', Progress in Human Geography, 21: 269-77.

Keller, H.H. and Hedley, M.R. (2002) 'Nutritional risk needs assessment of communityliving seniors: Prevalence of nutrition problems and priorities for action', Journal of Community Health, 27: 121.

Kilduff, A., McKeown, K. and Crowther, A. (1998) 'Health needs assessment in primary care - the evolution of a practical public health approach', Public Health, 112: $175-81$.

Kitzhaber, J.A. (1993) 'Prioritising health services in an era of limits: the Oregon experience', British Medical Journal, 307: 373-7.

Klein, R. (1998) 'Puzzling out priorities', British Medical Journal, 317: 959-60.

Lane, L. (1994) A healthy future: report on Wairoa District health and disability support services. Wellington: Central Regional Health Authority.

Le Grand, J., Mays, N. and Mulligan, J.A. (1998) Learning from the NHS Internal Market: A Review of the Evidence. London: King's Fund Institute.

Lenaghan, J. (1997) 'Central government should have a greater role in rationing decisions: the case for.' British Medical Journal, 314: 967-70.

Lincoln, Y.S. and Guba, E.G. (1985) Naturalistic Inquiry. London: Sage.

Lindblom, C. (1959) 'The science of muddling through', Public Administration Review, 19: 79-88.

Lindblom, C. (1980) The Policy-Making Process. Englewood Cliffs: Prentice Hall.

Liss, P.-E. (1993) Health Care Need. Aldershot: Avebury, Ashgate Publishing Ltd.

London Health Economics Consortium (1996) Local Health and the Vocal Communities - A review of developing practice in community based health needs assessment. London: Report commissioned by the London Primary Health Care Forum.

Lovatt, D. (1996) 'Lessons from contestability', Health Manager, 3: 12-5.

Manawatu-Wanganui Area Health Board (1991) Management Review of Tararua Health Services. Palmerston North: Manawatu-Wanganui Area Health Board.

Maori Health Operating Group-Health Funding Authority (1999a) Profile of the Auckland Locality. Wellington: Health Funding Authority.

Maori Health Operating Group-Health Funding Authority (1999b) Profile of the Bay of Plenty Locality. Wellington: Health Funding Authority. 
Maori Health Operating Group-Health Funding Authority (1999c) Profile of the Canterbury West Coast Locality. Wellington: Health Funding Authority.

Maori Health Operating Group-Health Funding Authority (1999d) Profile of the Nelson Marlborough Locality. Wellington: Health Funding Authority.

Maori Health Operating Group-Health Funding Authority (1999e) Profile of the Northland Locality. Wellington: Health Funding Authority.

Maori Health Operating Group-Health Funding Authority (1999f) Profile of the Otago Southland Locality. Wellington: Health Funding Authority.

Maori Health Operating Group-Health Funding Authority (1999g) Profile of the Tairawhiti Hawkes Bay Locality. Wellington: Health Funding Authority.

Maori Health Operating Group-Health Funding Authority (1999h) Profile of the Taranaki Locality. Wellington: Health Funding Authority.

Maori Health Operating Group-Health Funding Authority (1999i) Profile of the Waikato Locality. Wellington: Health Funding Authority.

Maori Health Operating Group-Health Funding Authority (1999j) Profile of the Wanganui Manawatu Locality. Wellington: Health Funding Authority.

Maori Health Operating Group-Health Funding Authority (1999k) Profile of the Wellington Locality. Wellington: Health Funding Authority.

Mays, N. and Hand, K. (2000) A Review of Options for Health and Disability Support Purchasing in New Zealand. Available at http://www.treasury.govt.nz/workingpapers/2000, accessed 20 June 2001.

Mays, N. and Pope, C. (1995) 'Qualitative research: rigour and qualitative research', British Medical Journal, 311: 109-12.

Mays, N. and Pope, C. (1997) Qualitative Research in Health Care. 1st Edition. London: BMJ Publishing Group.

Mays, N. and Pope, C. (2000) 'Assessing quality in qualitative research', British Medical Journal, 320: 50-2.

Mays N. et al (1998) What Were the Achievements of Total Purchasing Pilots in their First Year and How Can They be Explained? London: King's Fund Institute.

McEwen, J., Russell, E.M. and Stewart, S. (1995) 'Needs assessment in Scotland: collaboration in public health', Public Health, 109: 179-85.

McKean, W., Abernethy, P., Bobbett, M., Bohmer, P., Lock, P., Paul, A., Strang, C. and Watt, A. (1996) To spend or not to spend? That is the question: Resource allocation in the southern region - A strategy for change. Christchurch: Southern Regional Health Authority. 
McKee, M. and Figueras, J. (1996) 'Setting priorities: can Britain learn from Sweden?' British Medical Journal, 312: 691-4.

Mental Health Commission (1998) Blueprint for mental health services in New Zealand: how things need to be. Wellington: Mental Health Commission.

Metge, J. and Kinloch, B. (1984) Talking past each other: problems of cross-cultural communication. Wellington: Victoria University Press.

Miles, M.B. (1979) 'Qualitative data as an attractive nuisance: The problem of analysis', Administrative Science Quarterly, 24: 590-601.

Miles, M.B. and Huberman, A.M. (1994) Qualitative Data Analysis. 2nd Edn. Thousand Oaks, CA: Sage.

Milewa, T. (1997) 'Community participation and health care priorities: Reflections on policy, theatre and reality in Britain', Health Promotion International, 12: 161-8.

Miller, P., Parkin, D., Craig, N., Lewis, D. and Gerard, K. (1997) 'Less fog on the Tyne? Programme budgeting in Newcastle and North Tyneside', Health Policy, 40: 217-29.

Minister of Health (1995) Policy Guidelines for Regional Health Authorities 1996/97. Wellington: Ministry of Health.

Minister of Health (2000a) The Future Shape of Primary Health Care. Wellington: Ministry of Health.

Minister of Health (2000b) Governance of District Health Boards - Memorandum to Cabinet Social Policy and Health Committee, April 2000. Wellington: Office of the Minister of Health.

Minister of Health (2000c) Health and Disability Sector Changes and their Implementation - Memorandum to Cabinet Business Committee, January 2000. Wellington: Office of the Minister of Health.

Minister of Health (2000d) New Zealand Health Strategy. Wellington: Ministry of Health.

Minister of Health (2000e) New Zealand Public Health and Disability Act 2000. Wellington.

Minister of Health (2000f) The Role of District Health Boards and the Division of Functions between District Health Boards and the Ministry of Health - Memorandum to Cabinet Social Policy and Health Committee, April 2000. Wellington: Office of the Minister of Health.

Minister of Health (2001a) He Korowai Oranga: Maori Health Strategy: Discussion Document. Ministry of Health, Wellington.

Minister of Health (2001b) New Zealand Disability Strategy. Wellington: Ministry of Health. 
Minister of Health (2001c) The Primary Health Care Strategy. Wellington: Ministry of Health.

Minister of Health (2002) The Pacific Health and Disability Action Plan. Wellington: Ministry of Health.

Ministry of Health (1996) Progress on Health Outcome Targets. Wellington: Ministry of Health.

Ministry of Health (1997a) Korero Pasifika: consultation review making a Pacific difference. Wellington: Ministry of Health.

Ministry of Health (1997b) Making a Pacific difference: strategic initiatives for the health of Pacific people in New Zealand. Wellington: Ministry of Health.

Ministry of Health (1998a) 'Our children's health: key findings on the health of New Zealand children', in (eds), Wellington: Ministry of Health.

Ministry of Health (1998b) Progress on health outcome targets, Te haere whakamua ki nga whainga hua mo te hauora, 1997. Wellington: Ministry of Health.

Ministry of Health (1999a) Our health our future = hauora pakari, koiora roa: the health of New Zealanders, 1999. Wellington: Ministry of Health.

Ministry of Health (1999b) Our Health, Our Future. The Health of New Zealanders 1999. Wellington: Ministry of Health.

Ministry of Health (1999c) Progress on health outcome targets, Te haere whakamua ki nga whainga hua mo te hauora, 1998. Wellington: Ministry of Health.

Ministry of Health (1999d) Taking the Pulse. The 1996/97 New Zealand Health Survey. Wellington: Ministry of Health.

Ministry of Health (2000a) DHB Establishment Final Planning Guidelines. Ministry of Health, Wellington.

Ministry of Health (2000b) Health need assessment for New Zealand: An overview and guide. Wellington: Ministry of Health.

Ministry of Health (2001a) Operational Policy Framework 2001/2002. Ministry of Health, Wellington.

Ministry of Health (2001b) Planning Package for DHBs - DHB Accountability Indicators 2001/2002. Wellington: Ministry of Health.

Ministry of Health (2002a) Maori Health Strategy. Wellington: Ministry of Health.

Ministry of Health (2002b) Operational Policy Framework 2002/2003. Wellington: Ministry of Health. 
Ministry of Health (2002c) Reducing Inequalities in Health. Wellington: Ministry of Health.

Ministry of Health (2002d) Whakatataka: Maori Health Action Plan 2002-2005. Wellington: Ministry of Health.

Mitchell, G., Howden-Chapman, P., Bolevich, Z. and Smith, M. (2001) The Regional Health Needs Assessment Project. Health Services Research and Policy Conference, Wellington.

Mitchell, P. (1995) Otara Community Primary and Public Health Needs Assessment. Auckland.

Mooney, G. (1998) "Communitarian claims' as an ethical basis for allocating health care resources', Social Science and Medicine, 47: 1171-80.

Mooney, G., Haas, M., Viney, R. and Cooper, L. (1997) Linking Health Outcomes to Priority Setting, Planning and Resource Allocation. Sydney: Centre for Health Economics Research and Evaluation.

Mooney, G.H. (1977) 'Programme budgeting in an area health board.' The Hospital and Health Services Review: 379-84.

Mooney, G.H. (1984) 'Programme budgeting: an aid to planning and priority setting in health care.' Effective Health Care, 2: 65-8.

Mort, M., Harrison, S. and Dowswell, T. (Eds.) (1998) Public health panels in the UK: influence at the margins? London: Taylor and Wishart.

Murray, C.J.L. and Lopez, A.D. (Eds.) (1996) The Global Burden of Disease. The Harvard School of Public Health on behalf of the World Health Organisation and the World Bank, distributed by Harvard University Press.

Murray, S.A. (1999) 'Experiences with "rapid appraisal" in primary care: involving the public in assessing health needs, orientation staff, and educating medical students', British Medical Journal, 318: 440-4.

Murray, S.A. and Graham, L.J.C. (1995) 'Practice based health needs assessment: use of four methods in a small neighbourhood', British Medical Journal, 310: 1443-8.

Murray, S.A., Tapson, J., Turnbull, L., McCallum, J. and Little, A. (1994) 'Listening to local voices: adapting rapid appraisal to assess health and social needs in general practice', British Medical Journal, 308: 698-700.

National Advisory Committee on Core Health and Disability Support Services (1992) Core Health and Disability Support Services for 1993/1994 - First Report of the National Advisory Committee on Core Health and Disability Support Services. Wellington: National Advisory Committee on Core Health and Disability Support Services. 
National Advisory Committee on Core Health and Disability Support Services (1993) Disability support services: priorities: a Consensus Development Conference report to the National Advisory Committee on Core Health and Disability Support Services. Wellington: National Advisory Committee on Core Health and Disability Support Services.

National Advisory Committee on Core Health and Disability Support Services (1994) Core Services for 1995/96 - Third Report of the National Advisory Committee on Core Health and Disability Support Services. Wellington: National Advisory Committee on Core Health and Disability Support Services.

National Advisory Committee on Core Health and Disability Support Services (1995) Fourth Annual Report. Wellington: National Advisory Committee on Core Health and Disability Support Services.

National Forum on Health (1997) Research on Canadian values in relation to health and the health care system. Canada: Canadian Government.

National Health Committee (1999) Review of maternity services in New Zealand. Wellington: National Health Committee.

National Health Service Management Executive (1991) Assessing health care needs: a HA project discussion paper. London: Department of Health.

National Health Service Management Executive (1993) Health Service Indicators. Department of Health, London.

National Institute for Clinical Excellence (NICE) (2003) Technology Appraisals (Completed Appraisals). http://www.nice.org.uk/catta1.asp?c=153, accessed 26 April 2003.

Nevin, J.E. and Gohel, M.M. (1996) 'Community-orientated primary care', Primary Care, 23: 1-15.

New Zealand Government (2000) New Zealand Public Health and Disability Act 2000. Wellington.

NHS Executive (2003) Health needs assessment for PCTs. http://www.natpact.nhs.uk/competency framework/health improvement/Health nee ds_assessment/descriptors.php, accessed 26 April 2003.

NHS Management Executive (1992) Local voices. The views of local people in purchasing for health. London: Department of Health.

Nord, E., Richardson, J., Street, A., Kuhse, H. and Singer, P. (1995) 'Maximising health benefits vs. egalitarianism: an Australian survey of health issues', Social Science and Medicine, 41: 1429-37.

North Health (1996) Hauora Wahine Maori: A discussion document on Maori women's health. Auckland: North Health. 
North Health (1997) Pacific Islands People in the North Health Region. Auckland: North Health.

North Health (1998) Socio-economic inequalities in health care. Auckland: North Health.

Northern Regional Health Authority (1995) Maori in the North Health Region. Auckland: North Health.

Nuffield Institute of Health (University of Leeds), Centre for Health Economics (University of York) and Research Unit of the Royal College of Physicians (1993) Effectiveness of health care (nos. 1 to 7). Leeds: University of Leeds.

Nutting, P.A. and Connor, E.M. (1986) 'Community-orientated primary care: An examination of the US experience', American Journal of Public Health, 76: 279-81.

Nutting, P.A., Wood, M. and Conner, E.M. (1985) 'Community-orientated primary care in the United States', The Journal of the American Medical Association, 253: 1763-6.

Ong, B.N., Humphris, G., Annett, H. and Rifkin, S. (1991) 'Rapid appraisal in an urban setting, an example from the developed world', Social Science \& Medicine, 32: 90915.

Oregon Health Services Commission (1991) Prioritization of health services: a report to the Governor and the Legislature. Salem, Canada: Oregon Health Services Commission.

Organisation for Economic Co-operation and Development (1994) The Reform of Health Care Systems. Paris: OECD.

Orsman, B. (1999) Shipley attack blunted by hospital concerns. Weekend Herald, Auckland. p.A6, November 20-21.

Ovretveit, J. (1998) Evaluating Health Interventions. Buckingham: Open University Press.

Ovretveit, J.A. (1995) Purchasing for health. Buckingham: Open University Press.

Ovretveit, J.A. (1997) 'Managing the gap between demand and publicly affordable health care in an ethical way', European Journal of Public Health, 7: 128-35.

Oxford English Dictionary (2002) Concise Oxford English Dictionary. Tenth Edition. London: Oxford University Press.

Palmer, C.A. (1999) 'Rapid appraisal of needs in reproductive health care in southern Sudan: qualitative study', BMJ, 319: 743-8.

Parr, A., Whittaker, R. and Jackson, G. (1998) The Northern Region Health Survey 1996/97. Auckland: Health Funding Authority.

Parston, G. (1980) Planners, Politics and Health Services. London: Croom Helm. 
Patton, M.Q. (1990) Qualitative Evaluation and Research Methods. Newbury Park: Sage Publications.

Patton, M.Q. (1999) 'Enhancing the quality and credibility of qualitative analysis', Health Services Research, 34: 1189-1208.

Payne, J.N., Coy, J., Patterson, S. and Milner, P.C. (1994) 'Is use of hospital services a proxy for morbidity? A small area comparison of the prevalence of arthritis, depression, dyspepsia, obesity, and respiratory disease with inpatient admission rates for these disorders in England', Journal of Epidemiology \& Community Health, 48: 74-8.

Peacock, D., Devlin, N. and McGee, R. (1998) The horizontal equity of health care in New Zealand. Discussion Paper 9807. Dunedin: Economics Department, University of Otago.

Peacock, S., Richardson, J. and Carter, R. (1997) Setting priorities in South Australian community health II: marginal analysis of mental health services. West Heidelberg: Centre for Health Program Evaluation.

Petrou, S. (1998) 'Health needs assessment is not required for priority setting', British Medical Journal, 317: 1154.

Pharmaceutical Management Agency Ltd (2000) A prescription for pharmaceutical analysis. Wellington: PHARMAC.

Pope, C. and Mays, N. (1995) 'Qualitative research: Reaching the parts other methods cannot reach: an introduction to qualitative methods in health and health services research', British Medical Journal, 311: 42-5.

Porirua Community Health Group and Porirua Health Partnership (1997) Case Study: Maternity Services and Care in Porirua. Porirua: Porirua City Council.

Porirua Kapiti Healthlinks Project (2000a) Kapiti District Health and Disability Report and Plan. Wellington: Ministry of Health.

Porirua Kapiti Healthlinks Project (2000b) Porirua City Health and Disability Report and Plan. Wellington: Ministry of Health.

Public Health Commission (1993) Our health, our future = hauora pakari, koiora roa: The State of the public health in New Zealand 1993. Wellington: Public Health Commission.

Public Health Commission (1994a) Alcohol: The Public Health Commission's Advice to the Minister of Health 1993-1994. Wellington: Public Health Commission.

Public Health Commission (1994b) The health of Pacific Islands people in New Zealand. Wellington: Public Health Commission. 
Public Health Commission (1994c) Our health, our future = hauora pakari, koiora roa: The State of the public health in New Zealand 1994. Wellington: Public Health Commission.

Public Health Commission (1994d) Sudden Infant Death Syndrome (SIDS): The Public Health Commission's Advice to the Minister of Health 1993-1994. Wellington: Public Health Commission.

Public Health Commission (1995) Whakapiki mauri = Maori health advancement Wellington: Public Health Commission.

Public Health Consultancy (2001) An Assessment of Health Needs in the Lakes District Health Board Region: Te Tirohanga Hauora O Lakes (Summary Document). Rotorua: Lakes District Health Board.

Public Health Consultancy: Wellington School of Medicine and Health Sciences (2001) An assessment of health needs in the Wairarapa. Wellington: Wairarapa District Health Board.

Punch, K.F. (1998) Introduction to Social Research: Quantitative and Qualitative Approaches. London: Sage.

Robins, S.C. and Rigby, M.J. (1995) Electronic health records as a key to objective health care needs assessment beyond the hospital boundary. In MEDINFO 95 Conference, Edmonton, Canada.

Robinson, R. and Le Grand, J. (1994) Evaluating the NHS Reforms. London: King's Fund Institute.

Rossi, P.H., Freeman, H.E. and Lipsey, M.W. (1999) Evaluation: A Systematic Approach. Thousand Oaks: Sage.

Royal Commission on Social Policy (1988) Report of the Royal Commission on Social Policy. Wellington:

Ruta, D.A., Donaldson, C. and Gilray, I. (1996) 'Economics, public health and health care purchasing: the Tayside experience of programme budgeting and marginal analysis', Journal of Health Services Research \& Policy, 1: 185-93.

Sanderson, C.F.B., Hunter, D.J.W., McKee, M. and Black, N.A. (1997) 'Limitations of epidemiologically based needs assessment. The case of prostatectomy', Medical Care, 35: 669-85.

Scanlen, S. (1995) Whangarei area health and disability support services needs assessment. Whangarei.

Sceats, J., Hoskins, R., Moore, J. and O'Dea, D. (1995) 'One RHA's appproach to planning health services', Planning Quarterly, 118: 7-11.

Scott, C. (2001) Public and Private Roles in Health Care Systems. Wellington: Open University Press. 
Scott, C., Fougere, G. and Marwick, J. (1986) Choices for Health Care; Report of the Health Benefits Review. Wellington: Health Benefits Review.

Scottish Needs Assessment Programme (1998) Needs assessment in primary care: A rough guide. Glasgow: Scottish Needs Assessment Programme.

Secretary of State for Health (1997) The new NHS Modern and Dependable: A National Framework for Assessing Performance. Stationery Office, London.

Secretary of State for Health, W., Northern Ireland and Scotland, (1989) Working for patients: the health service: caring for the 1990s. HMSO, London.

Secretary of State for Social Services (1988) Public health in England: the report of the committee of inquiry into the future development of the public health function. HMSO, London.

Shanks, J., Kheraj, S. and Fish, S. (1995) 'Better ways of assessing health needs in primary care', British Medical Journal, 310: 480-1.

Simon, H.A. (1957) Administrative Behaviour. 2nd edn. New York: Macmillan.

Simpson, J. (1993) Primary mental health care: a discussion paper on current issues and service provision. Wellington: Department of Health.

South Australian Health Commission: South Australian Community Health Research Unit (1991) Planning healthy communities: A guide to doing community needs assessment. Bedford Park, South Australia: South Australian Health Commission.

Stake, R. (1995) 'Triangulation', in R. Stake (eds), The Art of Case Study Research. London: Sage Publications.

Stake, R.E. (1988) 'Case study methods in educational research: seeking sweet water', in R. M. Jaeger (eds), Complementary Methods for Research in Education. Washington, DC: American Educational Research Association.

Stevens, A. and Gabbay, J. (1991) 'Needs assessment needs assessment', Health Trends, 23: $20-3$.

Stevens, A. and Gillam, S. (1998a) 'The development of practical approaches to health needs assessment', in J. Wright (eds), Health Needs Assessment in practice. London: BMJ Books.

Stevens, A. and Gillam, S. (1998b) 'Needs assessment: from theory to practice', British Medical Journal, 316: 1448-52.

Stevens, A. and Raftery, J. (Eds.) (1994) Health care needs assessment: the epidemiologically based needs assessment reviews. Oxford: Radcliffe Medical Press.

Stevens, A. and Raftery, J. (Eds.) (1997) Health care needs assessment: the epidemiologically based reviews. Oxford: Radcliffe Medical Press. 
Swedish Parliamentary Priorities Commission (1995) Priorities in health care: Ethics, economy, implementation. Stockholm: Ministry of Health and Social Affairs.

Tollman, S. (1991) 'Community orientated primary care: origins, evolution, applications', Social Science and Medicine, 32: 633-42.

Toward, J.I. and Ostwald, S.K. (2002) 'Exploring mental health service needs for the elderly: results of a modified Delphi study', Community Mental Health Journal, 38: $141-9$.

Twaddle, S. and Walker, A. (1995) 'Programme budgeting and marginal analysis: application within programmes to assist purchasing in greater Glasgow Health Board', Health Policy, 33: 91-105.

Upton, S. (1991) Health Policy: Your Health and the Public Health: A statement of government health policy. Ministry of Health, Wellington.

Vaithianathan, R. (1996) Improving Health Purchasing: A Pilot in Public Health. Auckland: North Health.

Vedung, E. (1997) Public Policy and Programme Evaluation. New Brunswick: Transaction Publishers.

Wainwright, D. (1994) 'Needs Assessment. On the waterfront', Health Service Journal, 104: $28-9$.

Walt, G. (1994) Health policy: an introduction to process and power. London: Zed Books.

Wells, K., Klap, R., Koike, A. and Sherbourne, C. (2001) 'Ethnic disparities in unmet need for alcoholism, drug abuse, and mental health care', The American Journal of Psychiatry, 158: 2027-32.

Wilkin, D., Gillam, S. and Coleman, A. (2001) The National Tracker Survey of Primary Care Groups and Trusts 2000/2001: Modernising the NHS? Manchester: National Primary Care Research and Development Centre, University of Manchester.

Wilkinson, J. and Murray, S. (1998) 'Assessment in primary care: practical issues and possible approaches', British Medical Journal, 316: 1524-8.

Williams, A. (1999) 'Calculating the global burden of disease: time for a strategic appraisal?' Health Economics, 8: 1-8.

Williams, R. and Wright, J. (1998) 'Epidemiological issues in health needs assessment', British Medical Journal, 316: 1379-82.

Womersley, J. and McCauley, D. (1987) 'Tailoring health services to the needs of individual communities', Journal of Epidemiology \& Community Health, 41: 190-5.

Wood, G.D. (1981) 'The social and scientific context of rapid rural appraisal', IDS Bulletin, 12: 3-5. 
World Health Organisation (2003) WHO-CHOICE.

http://www3.who.int/whosis/menu.cfm?path=evidence,cea\&language=english, accessed 26 April 2003.

Wright, J. (Ed.) (1998) Health needs assessment in practice. London: BMJ Books.

Wright, J. and Walley, J. (1998) 'Assessing health needs in developing countries', British Medical Journal, 316: 1819-23.

Wright, J., Williams, R. and Wilkinson, J. (1998a) 'Development and importance of health needs assessment', British Medical Journal, 316: 1310-3.

Wright, J., Williams, R. and Wilkinson, J. (1998b) 'The development and importance of health needs assessment', in J. Wright (eds), Health Needs Assessment in Practice. London: BMJ Books.

Wright, R.A. (1993) 'Community-orientated primary care. The cornerstone of health care reform', The Journal of the American Medical Association, 269: 2544-7.

Yin, R.K. (1984) Case Study Research: Design and Methods. Newbury Park, CA: Sage. 